\title{
HUMAN RELIABILITY ANALYSIS FOR NMSS $\mathrm{P}-406$
}

MAY 2011
United States
Nuclear Regulatory Commission
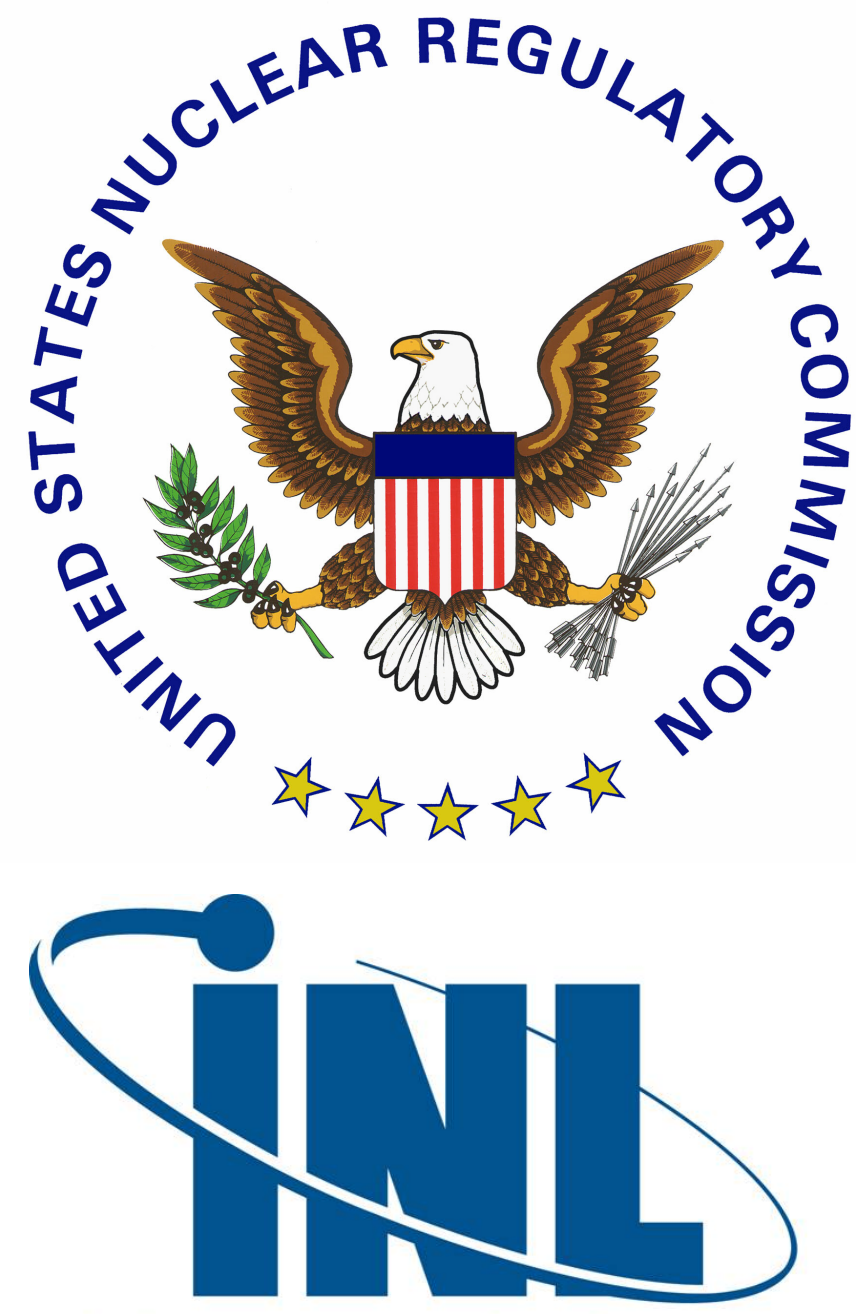

Idaho National Laboratory 


\section{DISCLAIMER NOTICE}

This report was prepared as an account of work sponsored by an agency of the United States Government. Neither the United States Government nor any agency thereof, or any of their employees, makes any warranty, expressed or implied, or assumes any legal liability of responsibility for any third party's use, or the results of such use, or any information, apparatus, product or process disclosed in this report, or represents that its use by such third party would not infringe privately owned rights. 


\section{TABLE OF CONTENTS}

\section{LESSONS}

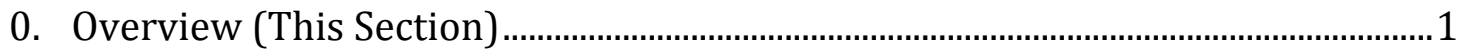

1. Introduction to Risk Assessment and Risk-Informed Decision Making................8

2. From Human Error to Human Reliability Analysis (HRA) .........................................

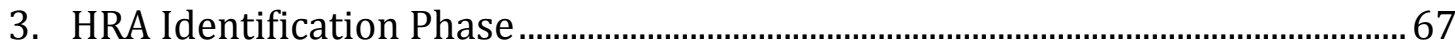

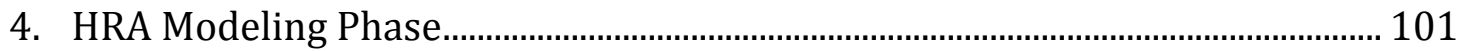

5. HRA Quantification Phase ………………………………………………………...... 131

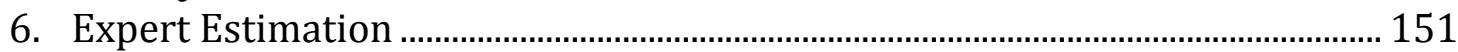

7. HRA Methods Overview ........................................................................................... 179

\section{APPENDICES}

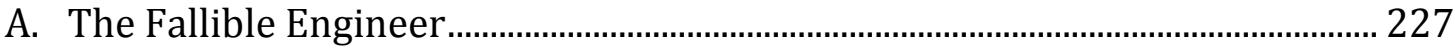
B. Medical Misadministration Example …………………………………………..... 234

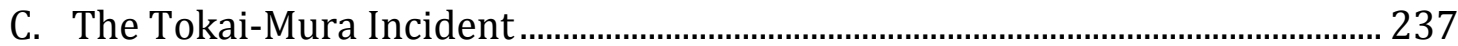
D. Spent Fuel Handling Example.................................................................................... 260

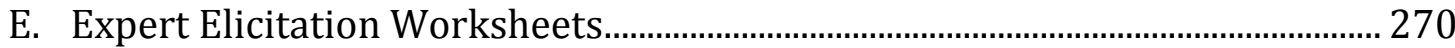
F. THERP Table 20 …………………………………………………………………... 280

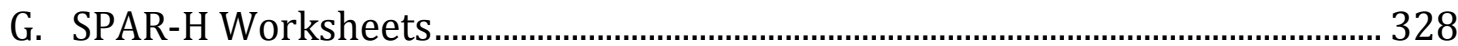
H. Article on Origins of SPAR-H Quantification ...........................................................340 



\section{P-406: Human Reliability Analysis for NMSS}

U.S. Nuclear Regulatory Commission May 2011

Instructors:

Ronald Boring, PhD

ronald.boring@inl.gov

David Gertman, PhD david.gertman@inl.gov 


\section{NRC PRA Program Overview}

The PRA Technology Training Program was established in 1982 to formulate a curriculum for training NRC staff members in the use of Probabilistic Risk Assessment (PRA) techniques. Use of these techniques enables the employee to evaluate risks associated with various safety issues, and to apply the results in the process of regulating and inspecting nuclear systems.

The training materials are periodically reviewed and revised to incorporate the latest information available, and as needs change, new courses and workshops are added to the curriculum. Course instructors are PRA experts employed in private industry and at the national laboratories. 


\section{NRC PRA Program Overview - cont.}

The PRA Technology Training Program is administered through the Associate Director for Training and Development (ADTD) of the Office of Human Resources.

The essential part of the PRA Training Program is meeting the needs of its users. In order to do so, we welcome your verbal and written feedback.

Please contact Russ Anderson, HR/ADTD/STS, 423-855-6519, or Michael Calley, INL, 208-526-9230, with your suggestions for the program. 


\section{Disclaimer}

The contents of this course documentation do not necessarily reflect the views or policies of the U.S. Nuclear Regulatory Commission, nor does mention of trade names, commercial products, or organizations imply endorsement by this or any other agency of the U.S. Government.

Neither the U.S. Government nor any of its agencies or employees makes any warranty, expressed or implied, or assumes any legal liability or responsibility for any third party use, or the results of such use, or any information, apparatus, product, or process disclosed in this document, or represents that any use by such third party would not infringe on privately owned rights. 


\section{Overview of Lessons}

0. Overview (This Section)

1. Introduction to Risk Assessment and Risk-Informed Decision Making

2. From Human Error to Human Reliability Analysis (HRA)

3. HRA Identification Phase

4. HRA Modeling Phase

5. HRA Quantification Phase

6. Expert Estimation

7. HRA Methods Overview 


\section{Overview of Appendices}

A. The Fallible Engineer

B. Medical Misadministration Example

C. The Tokai-Mura Incident

D. Spent Fuel Handling Example

E. Expert Elicitation Worksheets

F. THERP Table 20

G. SPAR-H Worksheets

H. Article on Origins of SPAR-H Quantification 


\section{Course Materials}

Textbooks

- James Reason, Human Error, Cambridge University Press, 1990.

- David I. Gertman \& Harold S. Blackman, Human Reliability \& Safety Analysis Data Handbook, Wiley Interscience, 1994.

- Textbook policy

- These stay in the classroom

- There are no required reading assignments, but you are encouraged to review these texts at your leisure

Supplemental CD

- Contains a number of significant NUREGs and other documents related to the course content (many not currently available on ADAMS)

- Open the CD and click on "index.htm" for an index of files

- Please take with you and put on your bookshelf for reference 


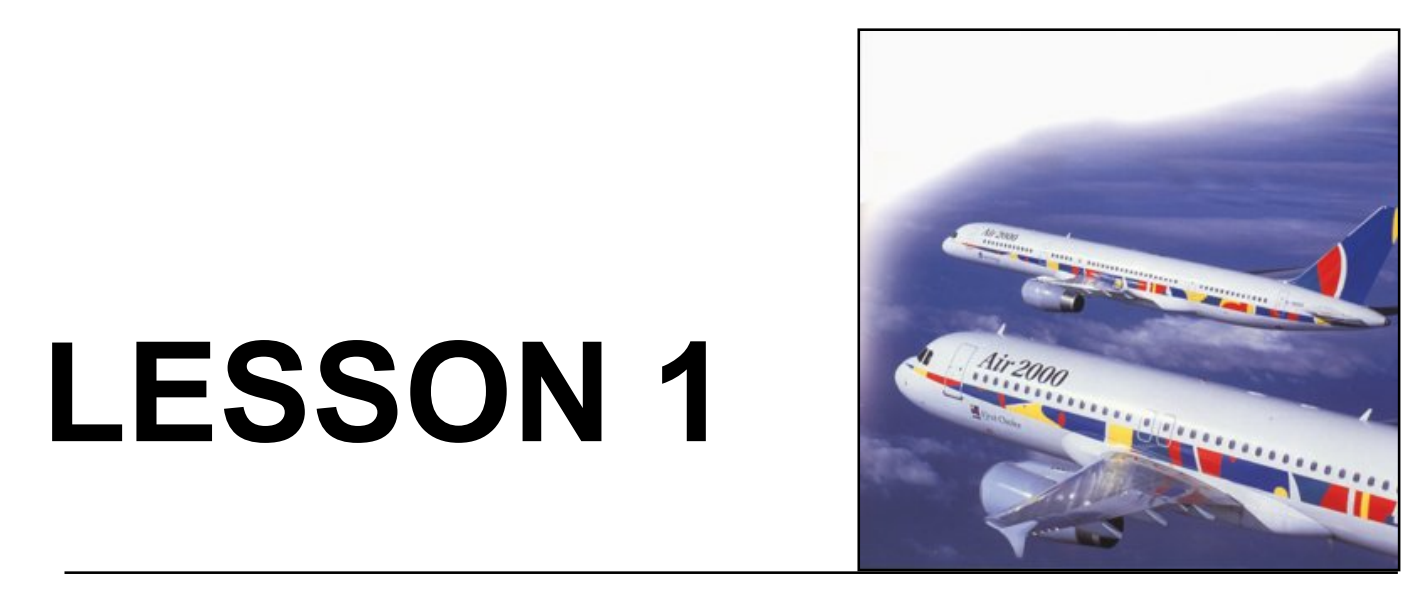

Introduction to Risk Assessment and Risk-Informed Decision Making 


\section{Lesson 1 Objectives}

- Review basic risk concepts

- Briefly introduce HRA as a field of risk

- Provide brief history of HRA

- Discuss risk-informed decision making 


\section{Reliability Engineering}

\section{Reliability $=$ Likelihood of Failure}

- A "high reliability" system is one that does not fail

- A "low reliability" system is one that does fail

- Most systems have a reliability lifecycle-a product life

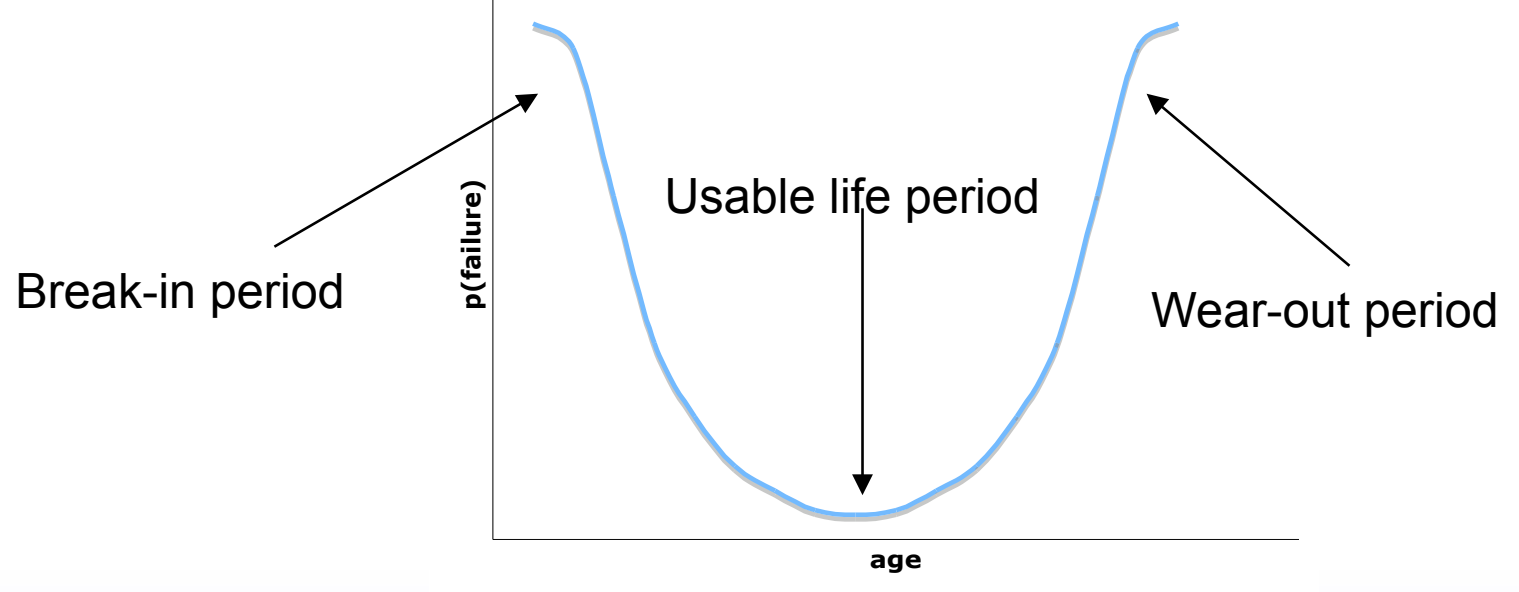




\section{Human Reliability Analysis?}

\section{How Does Human Reliability Relate?}

- Do we measure human reliability in terms of a break-in period, usable life period, and wear-out period?

- No! Humans are complex dynamic systems

- Machines don't have bad days-but humans do

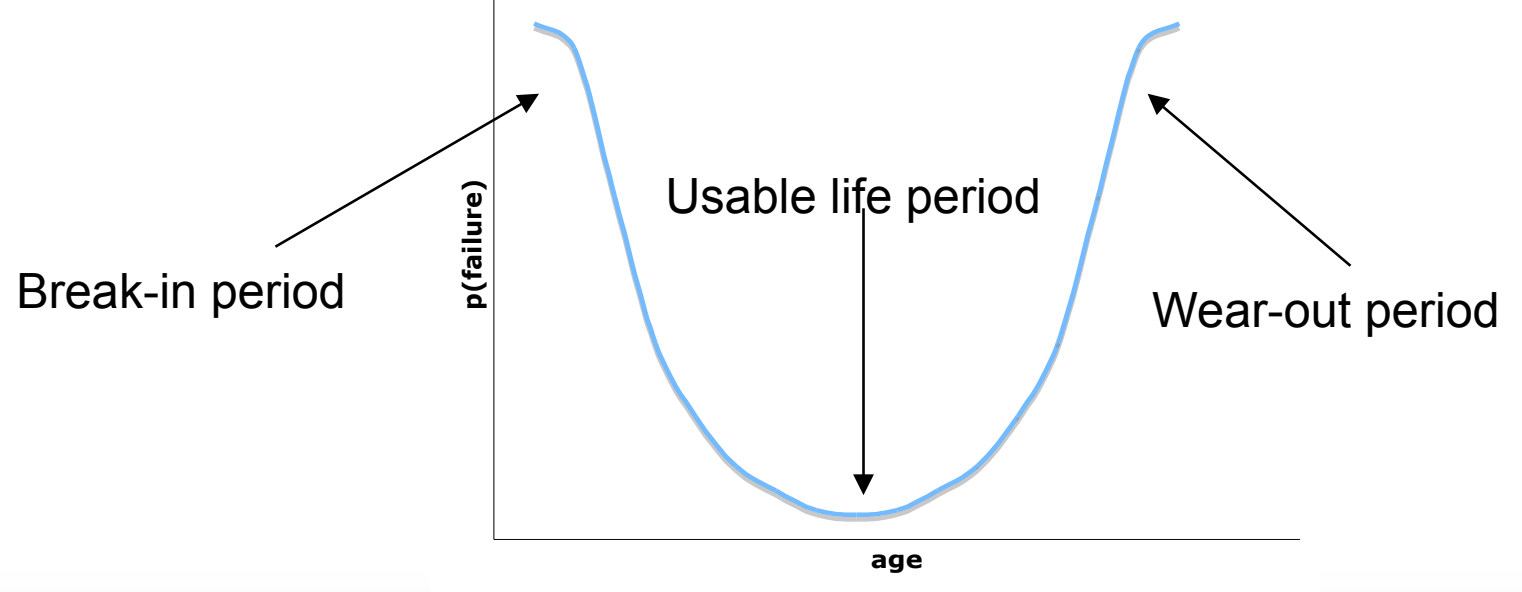




\section{A Day in the Life of A Human}

\section{Do Humans Have a Product Life?}

- We do have productive working years, but our reliability actually varies throughout the day

- Circadian rhythm-24-hour rest-wake cycle

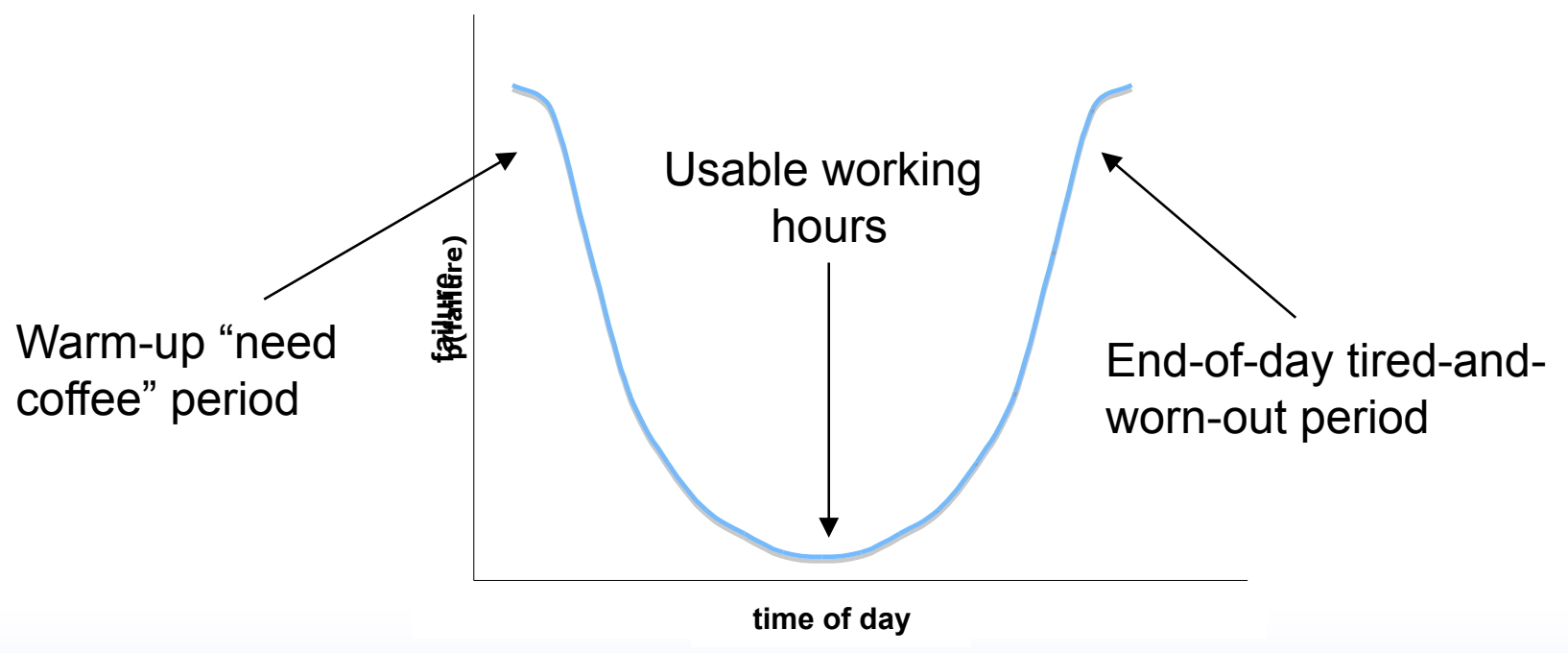




\section{Factors Affecting Human Reliability}

What Can Cause Humans to Perform Worse?

- What might increase the warm-up period?

- What might decrease working performance during day?

- What might increase end-of-day period?

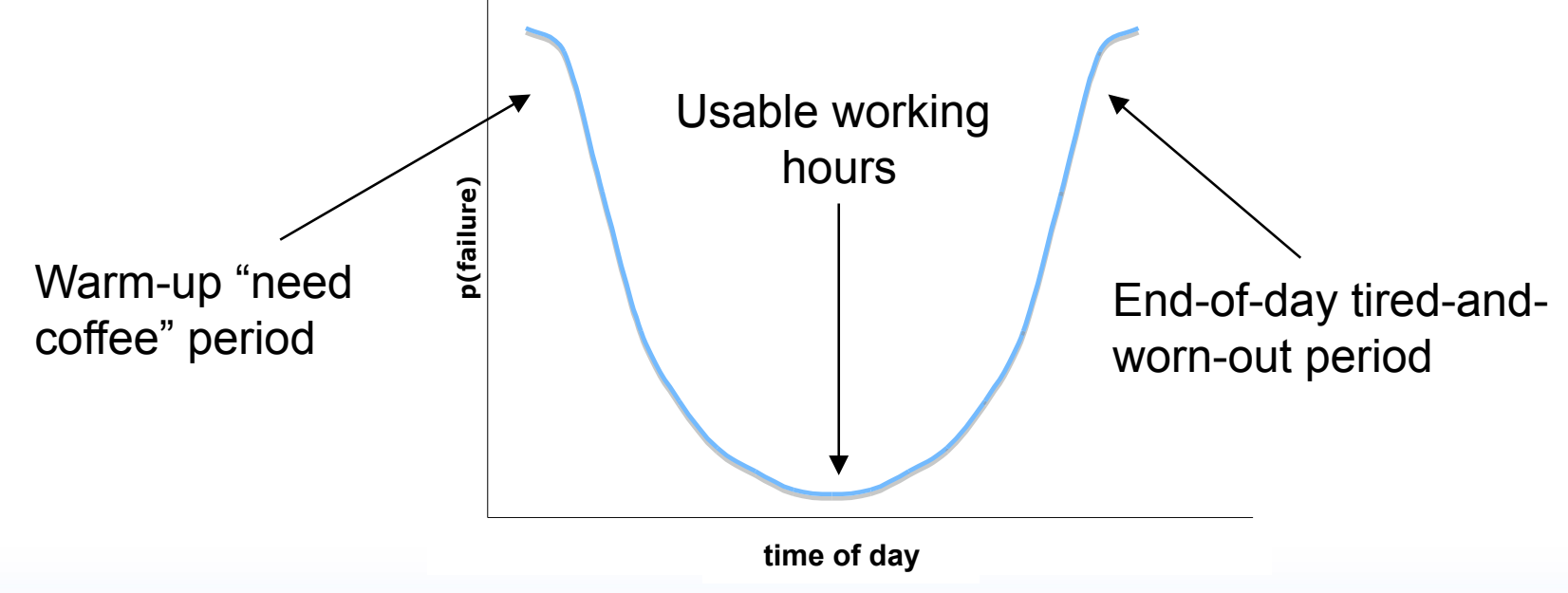




\section{Definitions}

Human Reliability Analysis (HRA) is:

- Study of human contribution to overall risk when interacting with a system

- Part of probabilistic risk assessment (PRA) that includes hardware and human reliability

- ASME RA-S-2002:

- "A structured approach used to identify potential human failure events and to systematically estimate the probability of those events using data, models, or expert judgment" 

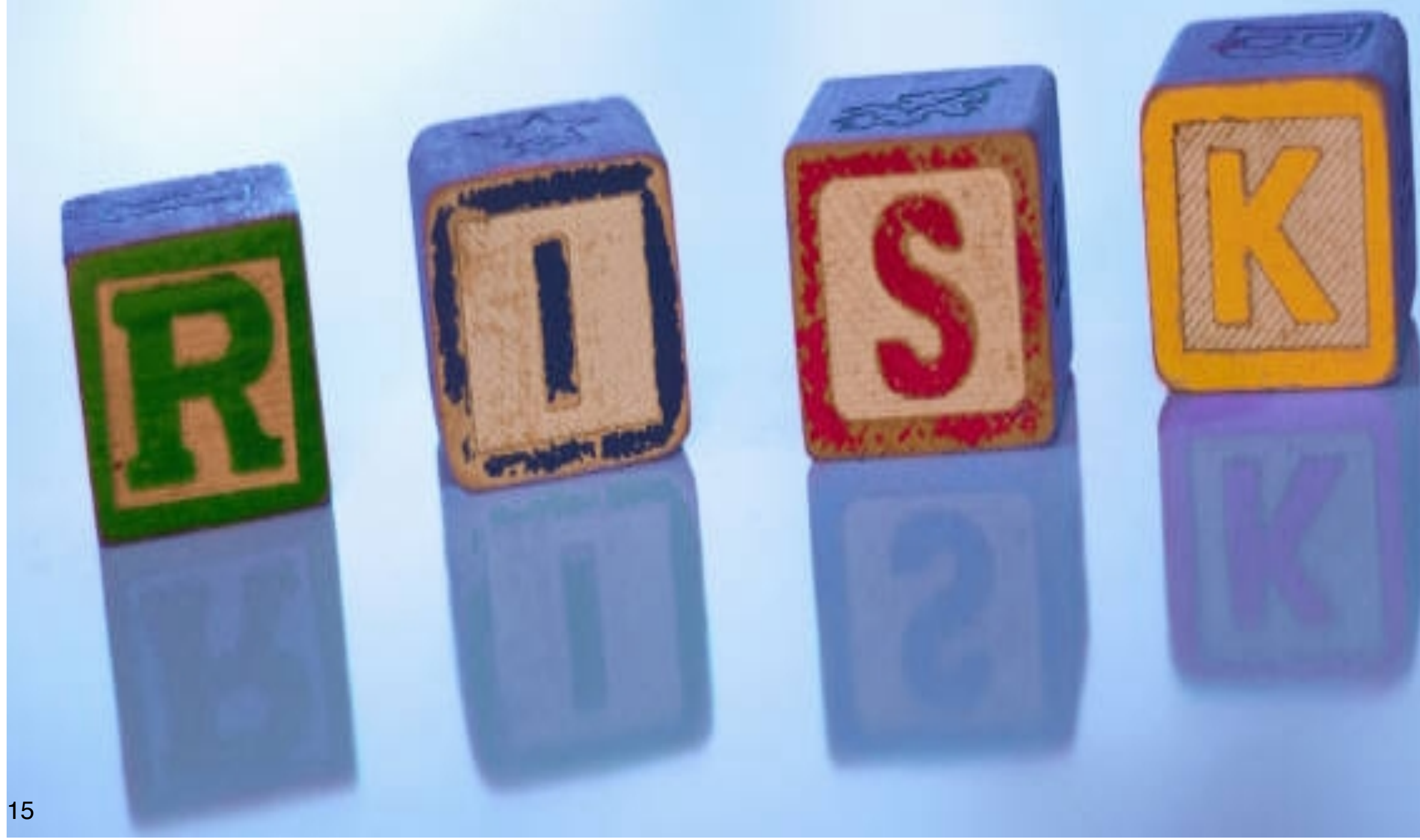


\section{What is Risk?}

\section{Definition of Risk}

- In the simplest of terms, risk is the likelihood of a hazard causing loss or damage

Risk is often framed in terms of the Risk Triplet:

- What can go wrong?

- How likely is it?

- What are the consequences? 


\section{What is Risk in Human Terms?}

\section{Definition of Risk}

- Risk is the likelihood of a human error causing loss or damage

\section{Definition of Human Error}

- Unwanted actions (or inactions) that deviate from expected and accepted courses of action

Human risk can also be framed in the Risk Triplet:

-What human actions can go wrong?

- How likely are these actions?

-What are the consequences of these actions? 


\section{HRA in Risk Assessment: The BIG Picture}

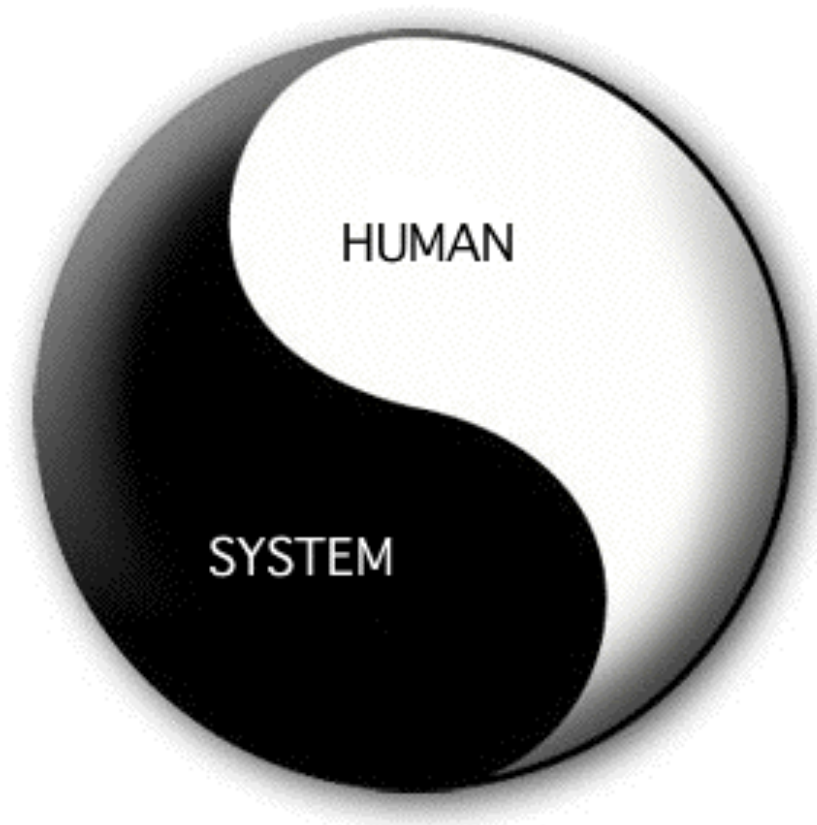

RISK
- Risk assessment looks at human-system activities and interactions and identifies the pathways by which the system mission might fail

- In a number of safety critical applications, people may actually be the predominant source of risk, not the system or hardware 


\section{Some Context}

PRA - Probabilistic Risk Assessment = Hardware and environmental contribution to risk

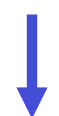

HRA - Human Reliability Analysis = Human contribution to risk

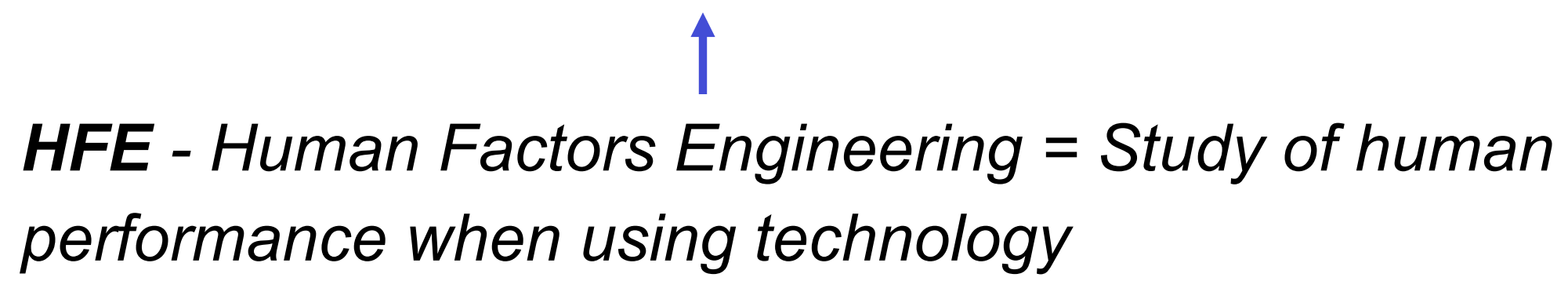




\section{Read and Discuss "The Fallible Engineer" (Appendix A)}

Discussion Topics

-What happened?

-Who was responsible?

-Where does human error occur?

- Who is to blame?

-What are the implications for reactors? 


\section{Risk is defined as the probability of an incident and its consequences}

\section{Risk Assessment}

Qualitative - identify possible human and hardware failure conditions

Quantitative - calculate probabilities of those failure conditions 


\section{Assessing Risk in the Old Days}
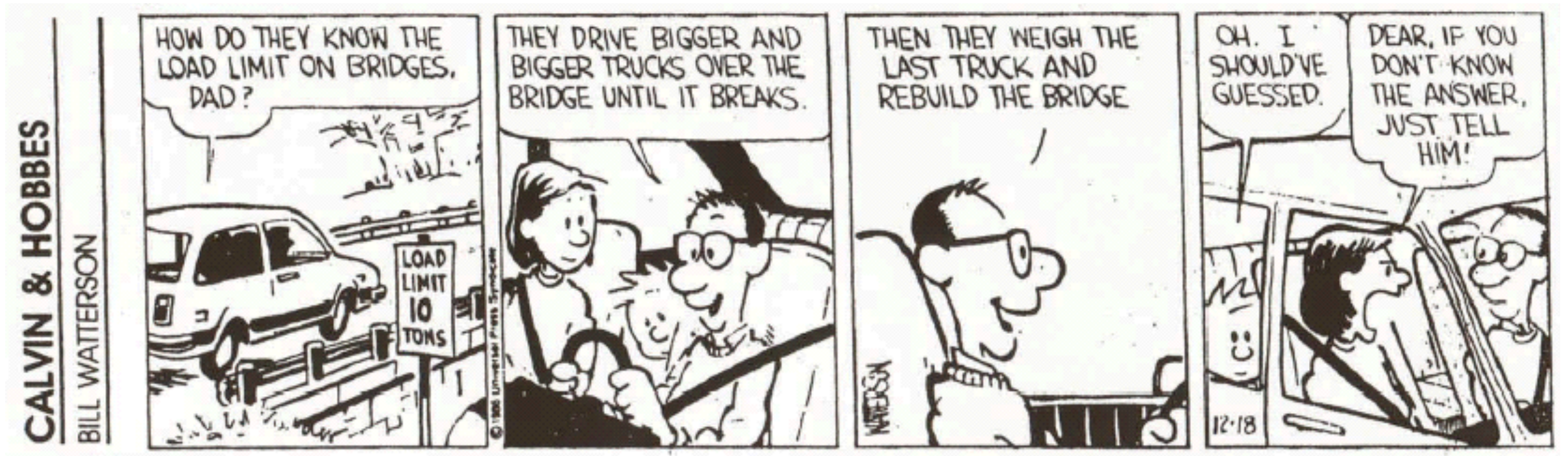


\section{Three Basic Phases of HRA}

HRA is a formal process to:

- Identify sources of human errors and error likely scenarios

- Model those human errors into an overall risk model

- Quantify Human Error Probabilities (HEPs)

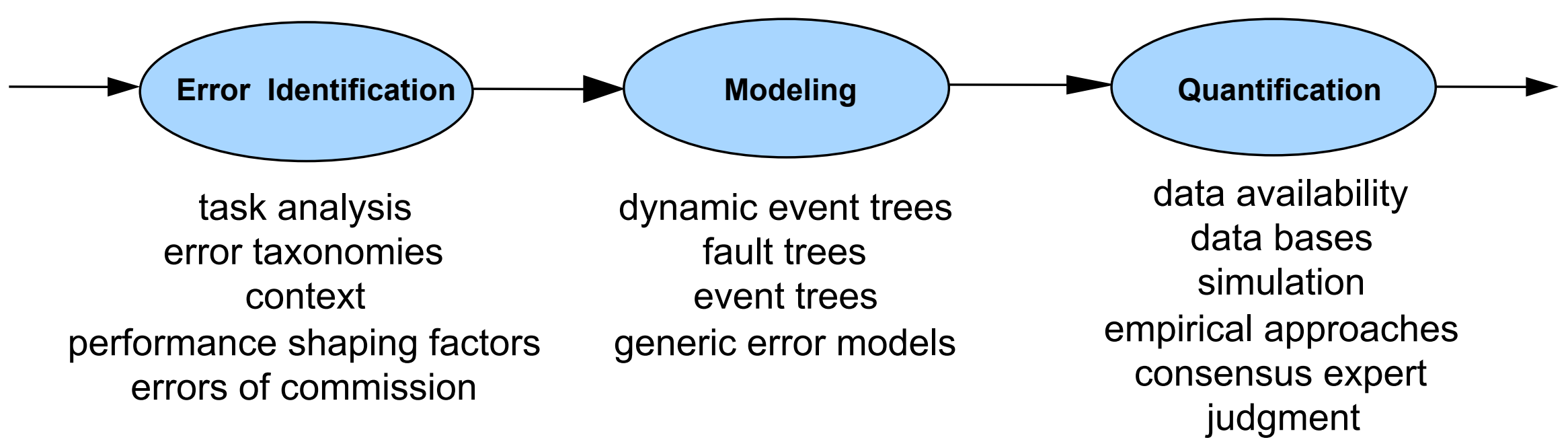




\section{Two Types of HRA}

\section{Retrospective HRA}

- Review previous incidents and determine the root cause of the incident in terms of human error

- Review the likelihood of the incident occurrence given the context and ways to prevent recurrence

- Example: Regulator review of licensee event

\section{Prospective HRA}

- Identify possible sources of human error in a system that has not been implemented or for an incident that has not been encountered

- Example: Licensee submittals for regulatory approval 


\section{History of HRA}

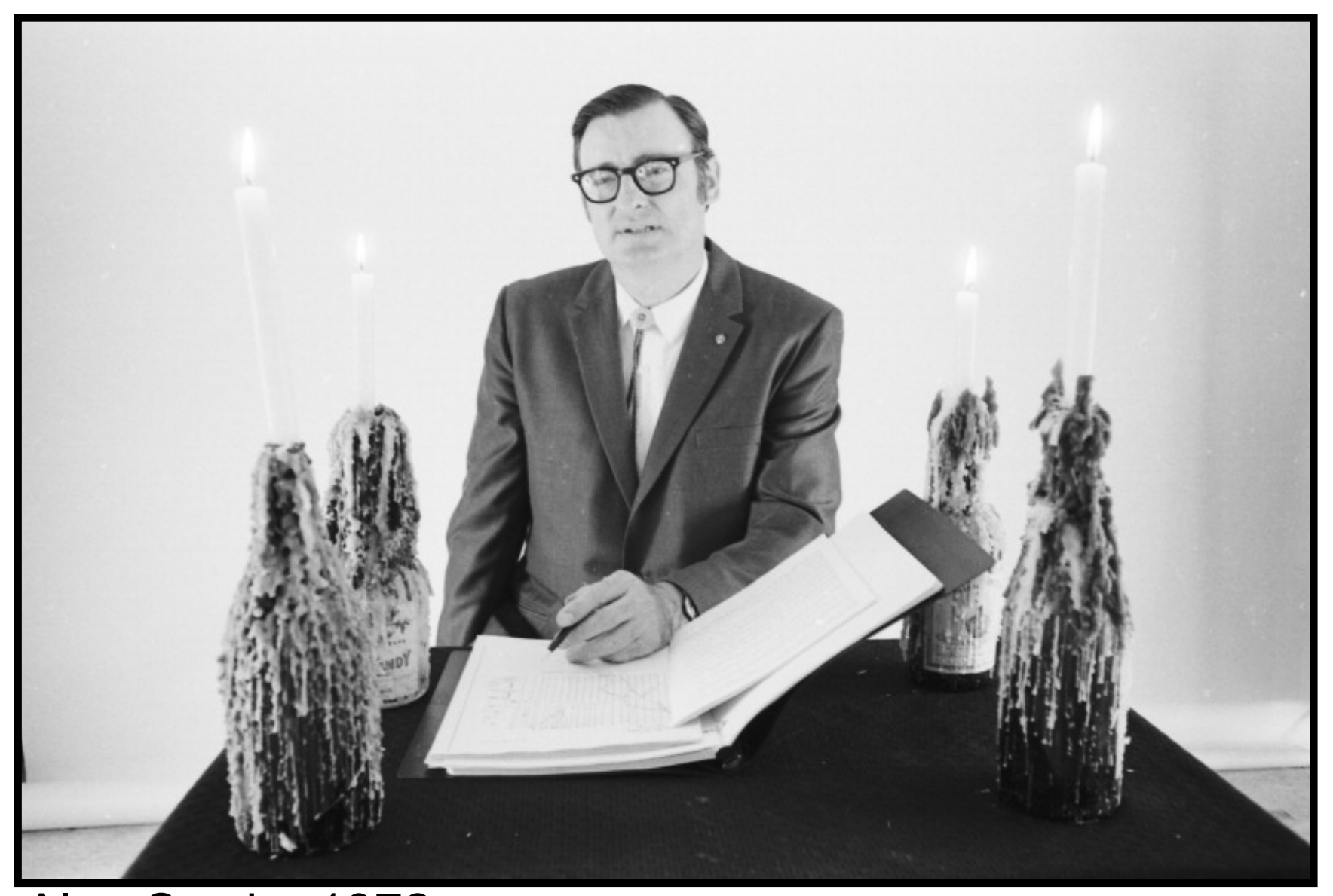

Alan Swain, 1972 


\section{History of HRA 1950 - 1970}

1950s - 1st HRA, Sandia National Lab. - studied human error in aircraft weapons systems; Sandia continued HRAs within nuclear weapons manufacturing \& handling

1962 - 1st human reliability data bank - AIR Data Store; 1st presentation of HRA to Human Factors Society

1964 - 1st HRA Symposium, Albuquerque

1967 - HRA technique accounts for dependencies between operators or tasks

1969 - USAF developed technique to model probability of error as a function of time, etc 


\section{History of HRA 1970 - 1990}

1970s - Development of THERP for nuclear power; use of simulator data

1980s - THERP revised, ASEP produced; new simulation models; concern over safety \& reliability of nuclear power industry (TMI); standardized HRA process; new HRA databases; new expert estimation techniques; increasing integration of HRAs into PRAs. Chernobyl typifies the role of human error in disaster. Recovery addressed

Modeling frameworks; Rasmussen: Skill-, Rule-, and Knowledge-based behavior; Reason: slips, lapses and mistakes

Time reliability correlation 


\section{History of HRA 1990 - present}

1990s - Consideration of management and organizational factors heightened, SPAR-H HRA method released, development of additional cognitive-oriented models including ATHEANA, CREAM, CAHR, HEART, MERMOS, HRA calculator, the investigation of work process (WPAM). IEEE STD 1082 (1997), ORE studies.

2000s - Compilation of HRA datasets for nuclear industry, aviation, and aeronautics. Application of ATHEANA. UK NARA effort. EPRI HRA Calculator, Application of HRA in support of NASA exploration. HRA Good Practices. Generalization of HRA results outside nuclear power industry. HRA benchmark. HERA database. Bayesian approaches explored. 


\section{HRA Methods Timeline}

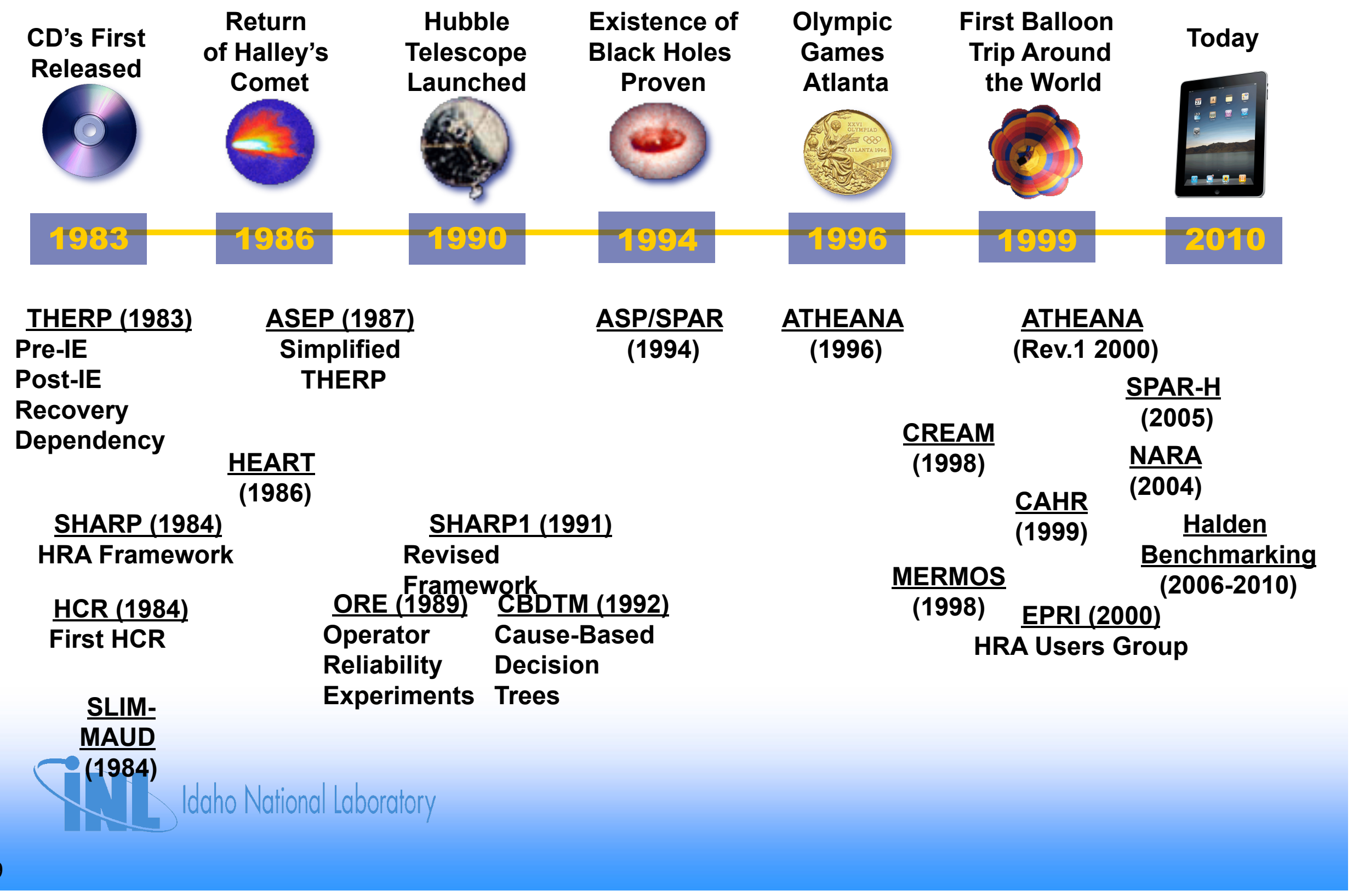




\section{Three Generations of HRA}

- Numerous distinctions have been posited

- The four classificatory Cs of generational HRA distinguish first and second generation HRA:

\begin{tabular}{|c|c|c|}
\hline Classification & 1G & 2G \\
\hline Cognition & $\times$ No & $\checkmark$ Yes \\
\hline Context & $\times$ No & $\checkmark$ Yes \\
\hline Commission & $\times$ No & $\checkmark$ Yes \\
\hline Chronology & $\times$ Older & $\checkmark$ Newer \\
\hline
\end{tabular}

- Dynamic modeling approaches have been suggested as the third generation 


\section{Risk-Informed Regulation}

- Insights derived from probabilistic risk assessments (PRAs), including HRAs, are used in combination with traditional engineering analyses to focus licensee and regulatory attention on issues commensurate with their importance to safety

- Various approaches are used in the resulting regulations:

- Prescriptive (e.g., design feature, program elements)

- Performance-oriented (e.g., maintenance rule, performance indicators/measures)

- Risk-oriented (e.g., Regulatory Guide 1.174) 


\section{Risk-Informed Regulation}

\section{Key Milestones at the NRC}

- WASH-1400 (NUREG-75/014) - Set framework for identifying quantitative measures of plant safety,

- Individual Plant Examination (Generic Letter 88-20)

- Licensee to identify and analyze plant safety vulnerabilities

- PRA Policy Statement (60 FR 42622, 16 August 1995)

- Improve regulatory decision making and therefore safety

- More efficient use of Staff resources

- Reduce regulatory burden on industry

- PRA Implementation Plan (SECY-99-211)

- Agency-wide plan to implement PRA Policy Statement for PRA-related activities

- Provide mechanisms for monitoring programs and oversight

- Risk-Informed Regulation Implementation Plan (13 Jan 2000 Memorandum)

- Organized to more broadly to track nuclear reactor, material, and waste safety

- Provide clear objectives and identify criteria for the selection and prioritization of practices and policies 


\section{Risk-Informed Regulation Implementation Plan (RIRIP) Suggests that PRA has Significant Benefits}

- Increased use of PRA can improve the regulatory process

- Increased use can promote regulatory efficiency

- "Living PRAs" can be of great value to licensees

- Analysis of PRA results can provide important safety insights 


\section{Areas Important to HRA in the RIRIP Are:}

- Medical applications

- Errors of commission

- Human performance data and database systems

- Organization and management issues 


\section{How is PRA Used in Risk-Informed Decision Making?}

- Determine system reliability

- Review system/plant design

- Improve system/plant availability

- Determine frequency of system/plant damage states

- Provide estimates of public risk

- Provide a basis for management and regulation

- Note that PRA includes human error as well as equipment unavailability 


\section{In Addition:}

- PRA is used to augment the NRC's traditional defensein-depth philosophy

- PRA insights may also be used to strengthen regulatory requirements where weaknesses are identified 


\section{LESSON 2}

\section{From Human Error to HRA}

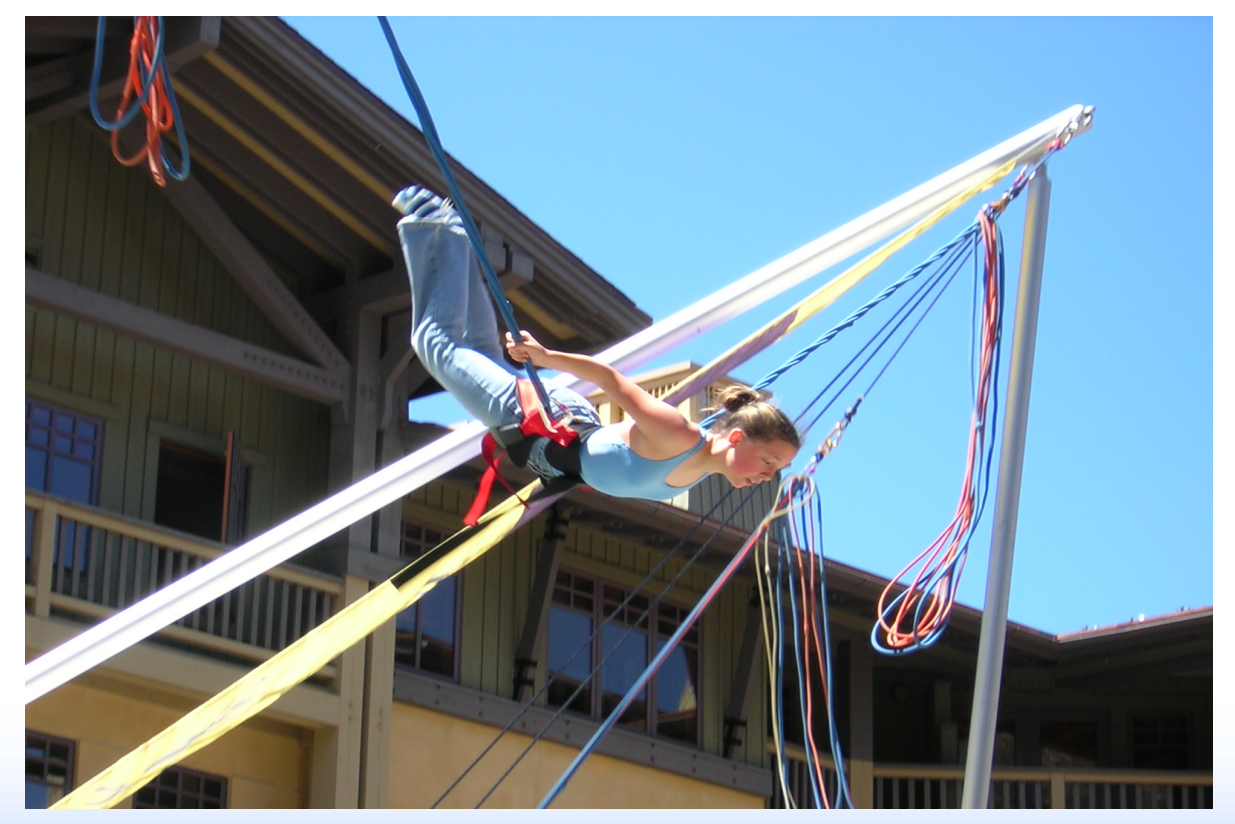




\section{Lesson 2 Objectives}

- Introduce basic concepts of human error

- Help you think about human error in terms of its psychological causes

- NOTE: The in-class exercises are not, strictly speaking, examples of human errors in nuclear power settings. The examples are kept simple enough to illustrate the basic concepts.

- Introduce active and latent errors

- Introduce the concept that a diagnosis of human error is only the starting point of an investigation, not the end point

- Briefly introduce safety culture 
What do we mean by human error?

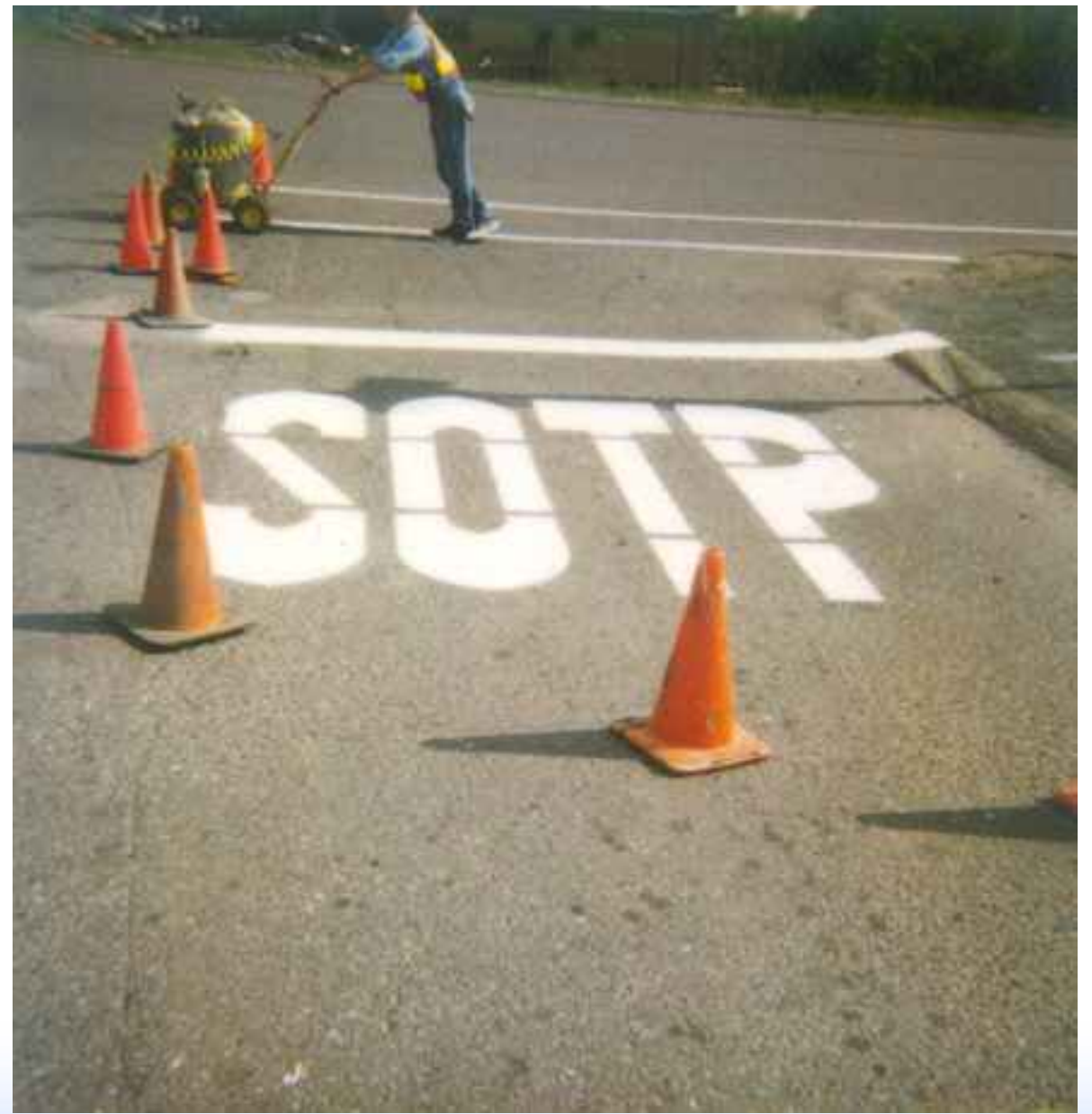




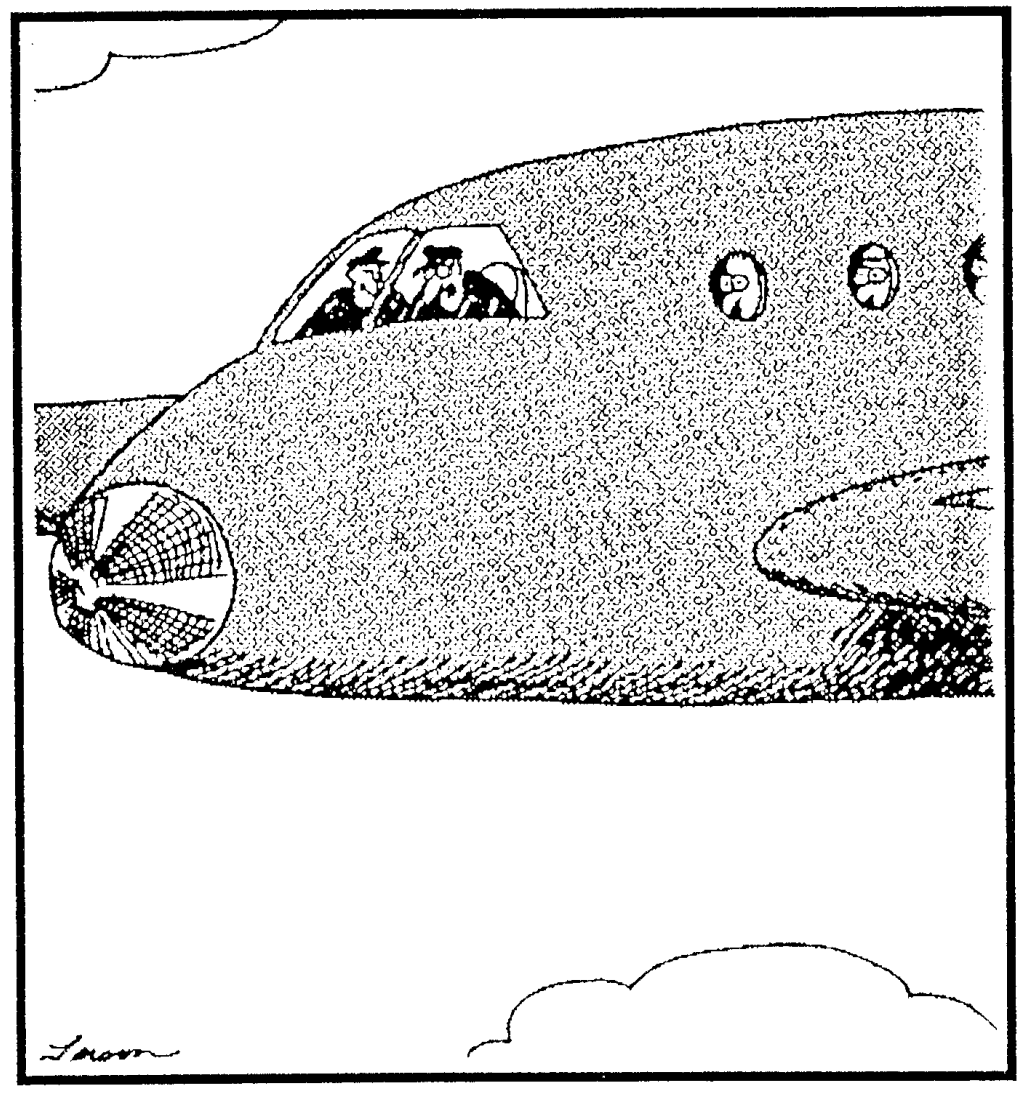

"The fuel light's on, Frank! We're all going to die!...We're all going to die!..Wait, wait...Oh, my mistake - that's the intercom light." 


\section{What is Human Error?}

- Unwanted actions or inactions that arise from problems in sequencing, timing, knowledge, interfaces, and/or procedures that result in deviations from expected standards or norms that places people, equipment, and systems at risk.

or

- A failure on the part of the human to perform a prescribed act (or performance of a prohibited act) within specified limits of accuracy, sequence or time, which could result in damage to equipment, or property, or disruption of schedules operations.

or

- An out of tolerance action, or deviation from the norm, where the limits of acceptable performance are defined by the system.

or

- Actions inappropriately taken, or not taken when needed, by plant personnel that result in a degraded plant safety condition. 


\section{Simple Definition of Human Error}

Human Error - Unwanted actions or inactions that result in deviations from expected standards or norms and that potentially place people, equipment, and systems at risk 


\section{Human Error $=$ Human Failure}

- In the PRA community, the term "human error" has often been used to refer to human-caused failures of systems or components

- However, in the behavioral sciences, the same term is often used to describe the underlying psychological failures that may cause the human action that fails the equipment

- Therefore, the term human error is only used in a very general way, with the terms human failure event and unsafe action being used to describe more specific aspects of human errors

- Human Failure Event (HFE)

- A basic event that is modeled in the logic models of a PRA (event and fault trees), and that represents a failure of a function, system, or component that is the result of one or more unsafe actions

- Unsafe Action (UA)

- Actions inappropriately taken, or not taken when needed, by plant personnel that result in a degraded plant safety condition. 
Exercise: How many f's?

\section{Finished Files are the Result of Years of Scientific Study Combined With the Experience of Many Years.}


Classroom Exercise: Read the three phrases. 


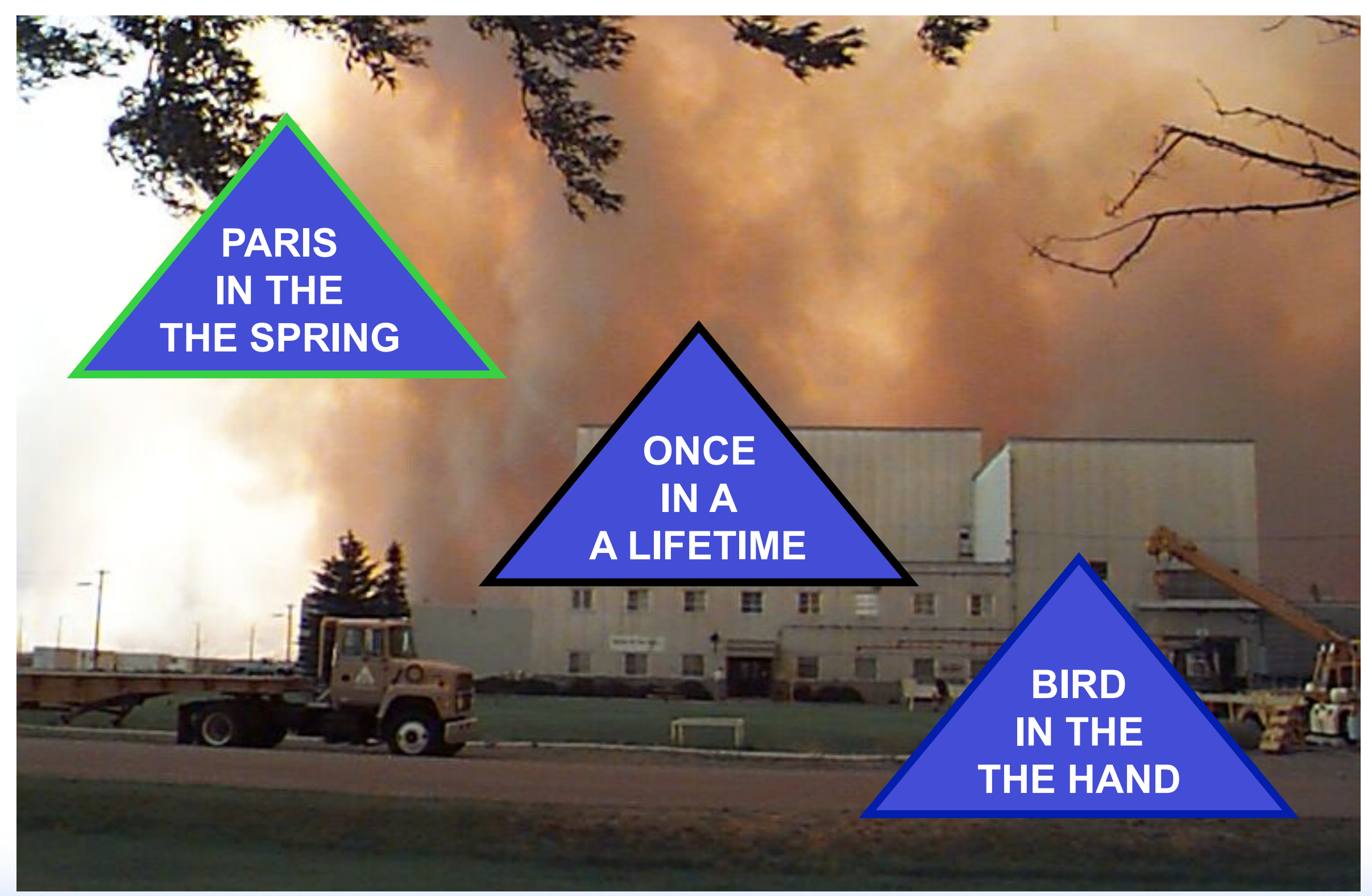

Idaho National Laboratory 
Aoccdrnig to a rscheearch at Cmabrigde Uinervtisy, it deosn't mttaer in waht oredr the Itteers in a wrod are, the olny iprmoetnt tihng is taht the frist and Isat Itteer be at the rghit pclae. The rset can be a toatl mses and you can sitll raed it wouthit porbelm. Tins is bcuseae the huamn mnid deos not raed ervey Iteter by istlef, but the wrod as a wlohe.

For a better explanation, see:

http://www.mrc-cbu.cam.ac.uk/ mattd/Cmabrigde/ 


\section{Human Error is Everywhere}

Even routine tasks like reading, writing, and speaking are extremely error prone

- The propensity to commit errors is further increased in complex tasks requiring extensive training, expertise, and procedural compliance

\section{Humans are resilient}

- Even though we commit errors frequently, most are inconsequent

- A stumble in my speech does not prevent you from understanding what I am saying from the context of the rest of the sentence

- Many potentially consequential errors are spontaneously recovered

- We self-check and correct errors

- Safety systems or others "catch" the errors and help us correct them 


\section{Human Error is a Significant Contributor to Risk}

$\rightarrow \quad$ Accidents at Sea

Chemical Industry

Airline Industry

Commercial Nuclear Industry
$90 \%$

$80-90 \%$

$60-87 \%$

$65 \%$

From: D.I. Gertman \& H.S. Blackman, Human Reliability \& Safety Analysis Data Handbook, Wiley-Interscience, 1994. 


\section{Human error has been shown to contribute}

from 50 to $70 \%$ of the risk at nuclear power plants

From: T.A. Trager, Jr., Case Study Report on Loss of Safety System Function Events, AEOC/C504, US NRC, 1985. 


\section{Importance of Human Error in Risk From NUREG/CR-6753 (2002)}

\begin{tabular}{|l|l|l|l|l|l|l||}
\hline $\begin{array}{l}\text { Power } \\
\text { Plant }\end{array}$ & $\begin{array}{l}\text { Event } \\
\text { Date }\end{array}$ & $\begin{array}{l}\text { LER } \\
\text { Number }\end{array}$ & $\begin{array}{l}\text { SPAR } \\
\text { Analysis } \\
\text { CCDP }\end{array}$ & $\begin{array}{l}\text { Risk Factor } \\
\text { Increase } \\
\text { (CCDP/CDP) }\end{array}$ & $\begin{array}{l}\text { Event } \\
\text { Importance } \\
\text { (CCDP- } \\
\text { CDP) }\end{array}$ & $\begin{array}{l}\text { Human } \\
\text { Error } \\
\text { Percent } \\
\text { Contribution } \\
\text { to Event } \\
\text { Importance }\end{array}$ \\
\hline $\begin{array}{l}\text { Wolf } \\
\text { Creek 1 }\end{array}$ & $1 / 30 / 96$ & $\begin{array}{l}482 / 96- \\
001\end{array}$ & $5.2 \mathrm{E}-03$ & 24,857 & $5.2 \mathrm{E}-03$ & 100 \\
\hline $\begin{array}{l}\text { Indian } \\
\text { Point 2 }\end{array}$ & $8 / 31 / 99$ & $\begin{array}{l}\text { AIT 50- } \\
246 / 99- \\
08\end{array}$ & $3.5 \mathrm{E}-04$ & 25 & $3.4 \mathrm{E}-04$ & 100 \\
\hline $\begin{array}{l}\text { McGuire } \\
2\end{array}$ & $12 / 27 / 93$ & $\begin{array}{l}370 / 93- \\
008\end{array}$ & $4.6 \mathrm{E}-03$ & 2.4 & $2.7 \mathrm{E}-03$ & 82 \\
\hline $\begin{array}{l}\text { Haddam } \\
\text { Neck }\end{array}$ & $6 / 24 / 93$ & $\begin{array}{l}213 / 93- \\
006 \text { \& - } \\
007 \text { AIT } \\
213 / 93- \\
80\end{array}$ & $2.0 \mathrm{E}-04$ & 4.3 & $1.5 \mathrm{E}-04$ & 48 \\
\hline
\end{tabular}




\section{Different Errors Contribute to Failure}

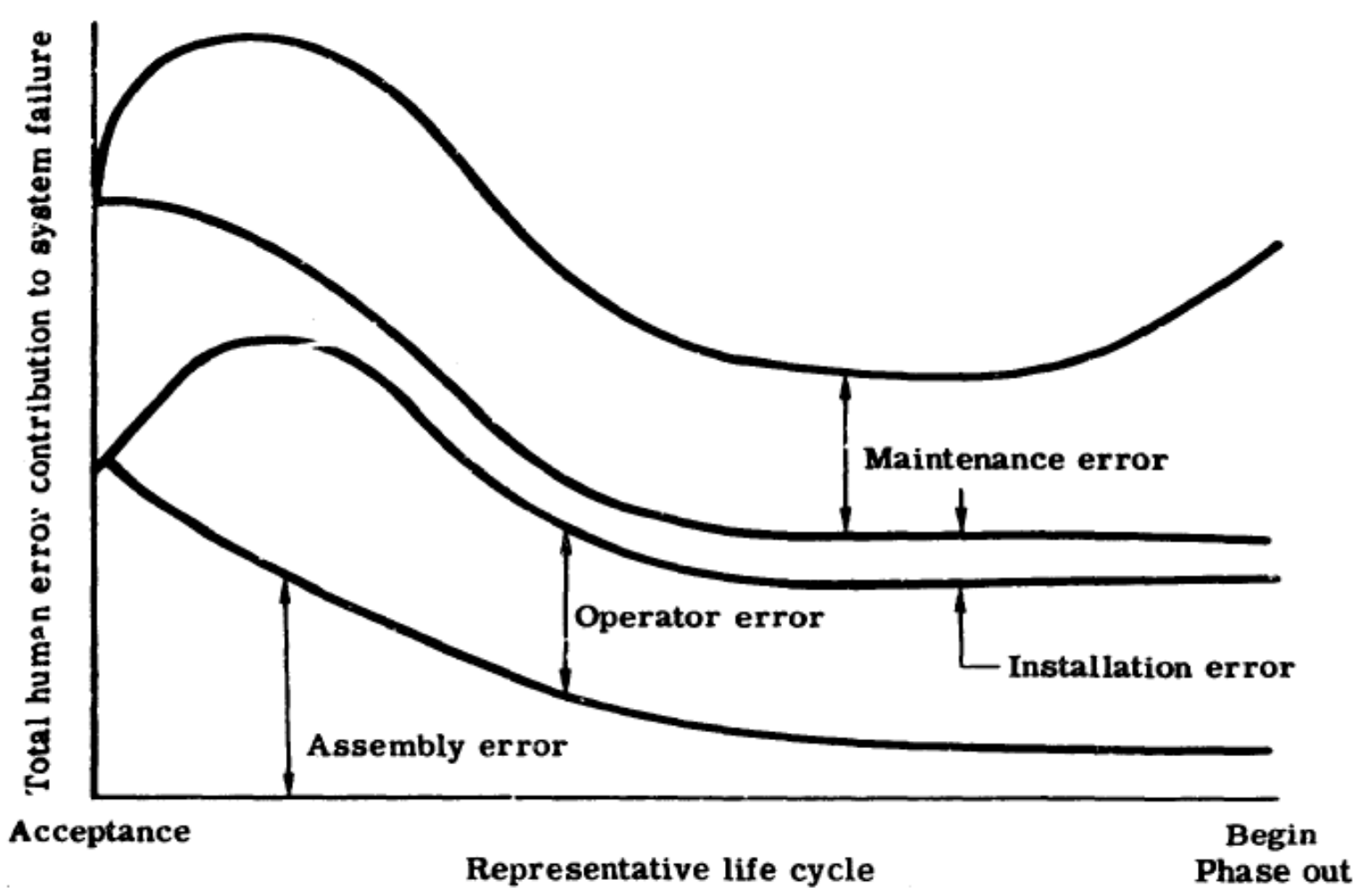

Proportional contribution of the different types of human error to overall failure (Rigby, 1967) 


\section{Types of Human Error Identified in Augmented Inspection Teams (AIT) Reports}

Human Error Type

Procedures

Training

Supervision

Human Engineering

Communications

Management \& Organization

Individual Issues

Workload

System Design

Work Environment

AIT (40 teams)
$65 \%$
$40 \%$
$43 \%$
$40 \%$
$35 \%$
$83 \%$
$38 \%$
$10 \%$
$58 \%$
$8 \%$




\section{Errors Can Occur Across Plant Operations}

NUREG-1774 chronicles crane operations from 1968 - 2002

- An average of $73 \%$ of incidents involved human performance

- Is the human performance component increasing?

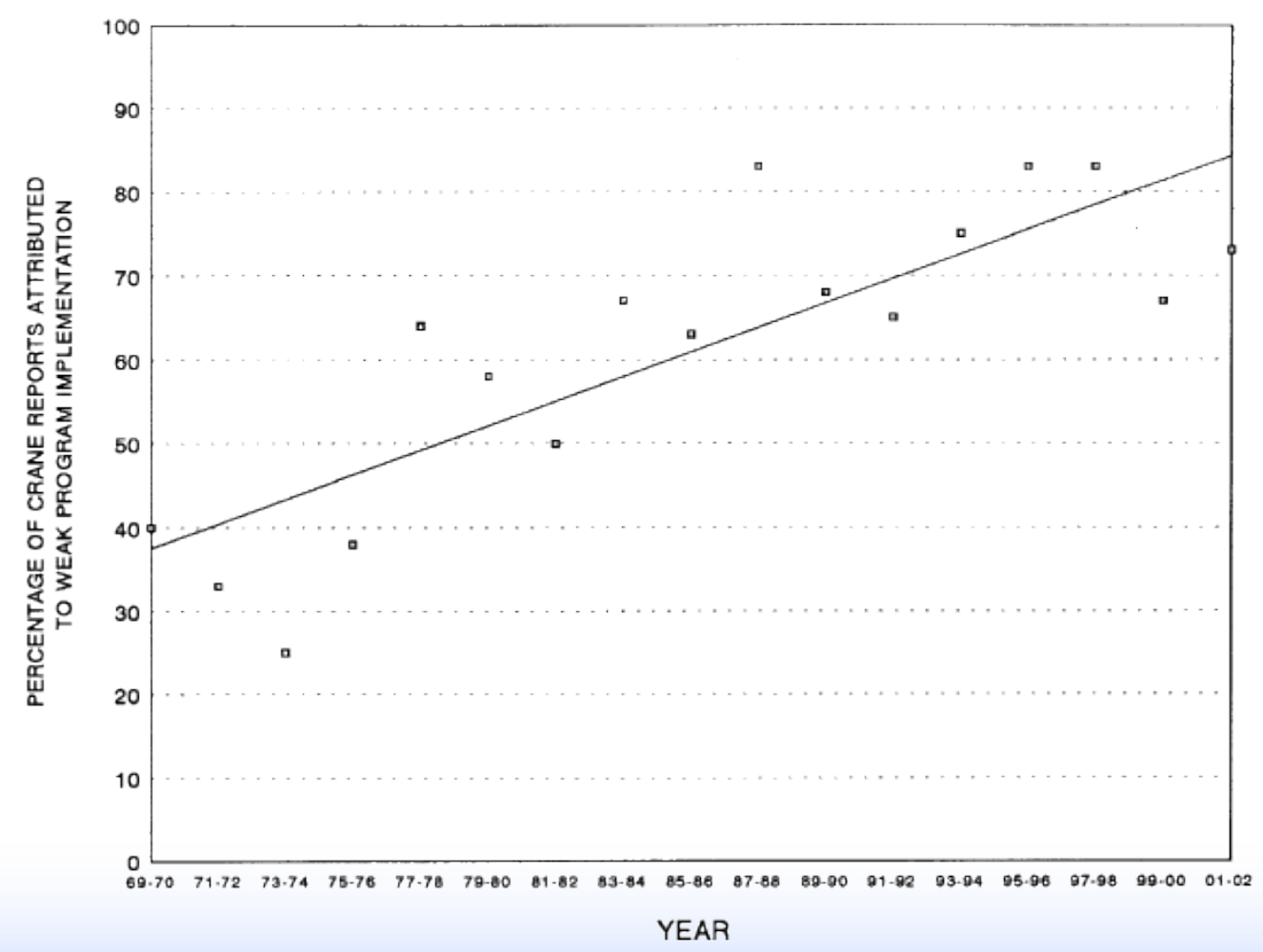




\section{Human Errors in Crane Operations}

Largest human contributors to crane events in NUREG-1774

- Not following procedures

- Failure to establish the required ventilation prior to load movements in certain areas

- Failure to perform crane surveillance tests prior to use

- Failure to move loads over established safe load path areas

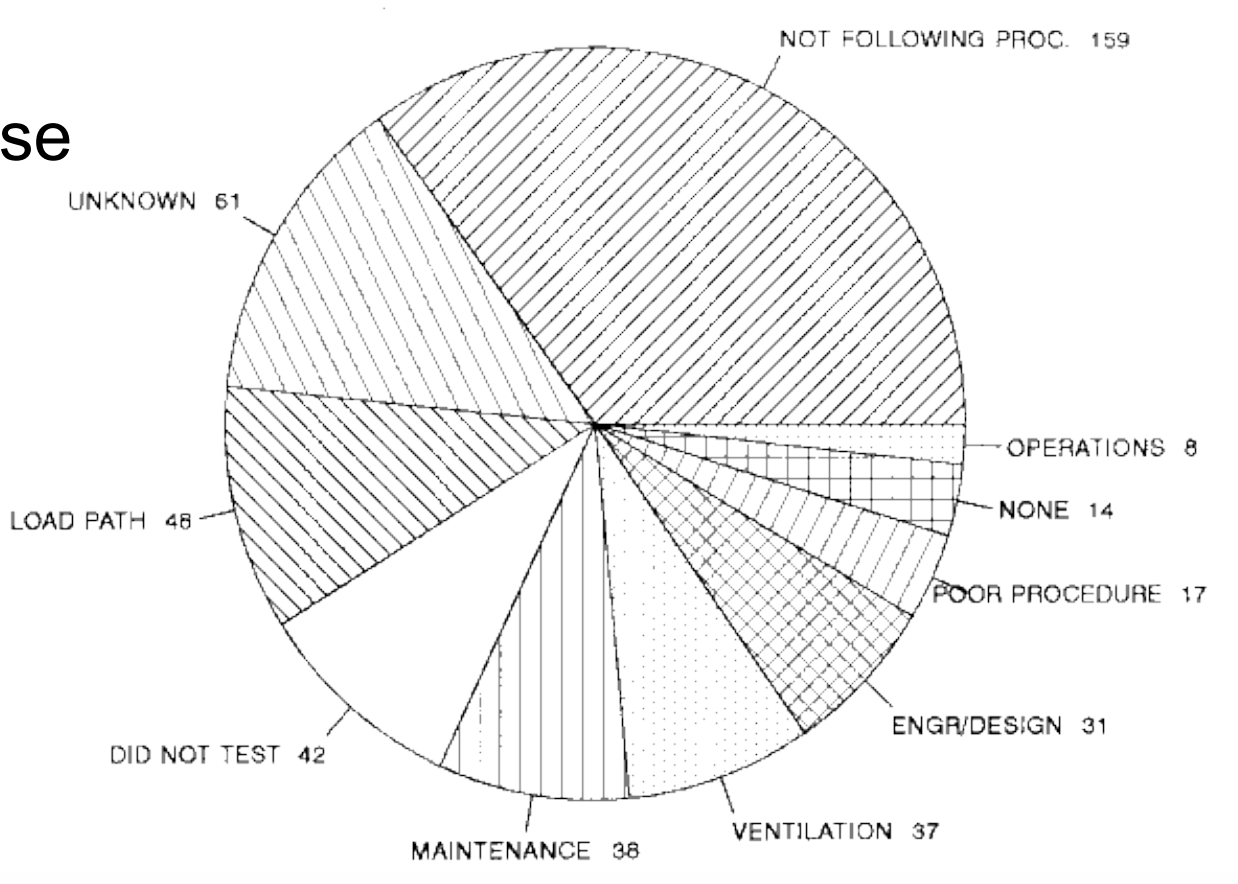

Figure 6: Principal reasons for crane events 


\section{Active Versus Latent Errors}

\section{Active Error $\longleftarrow$}

Active Errors are unsafe acts, failures of technological functions or human actions, which become the local triggering events that afterwards are identified as the immediate causes of an accident.
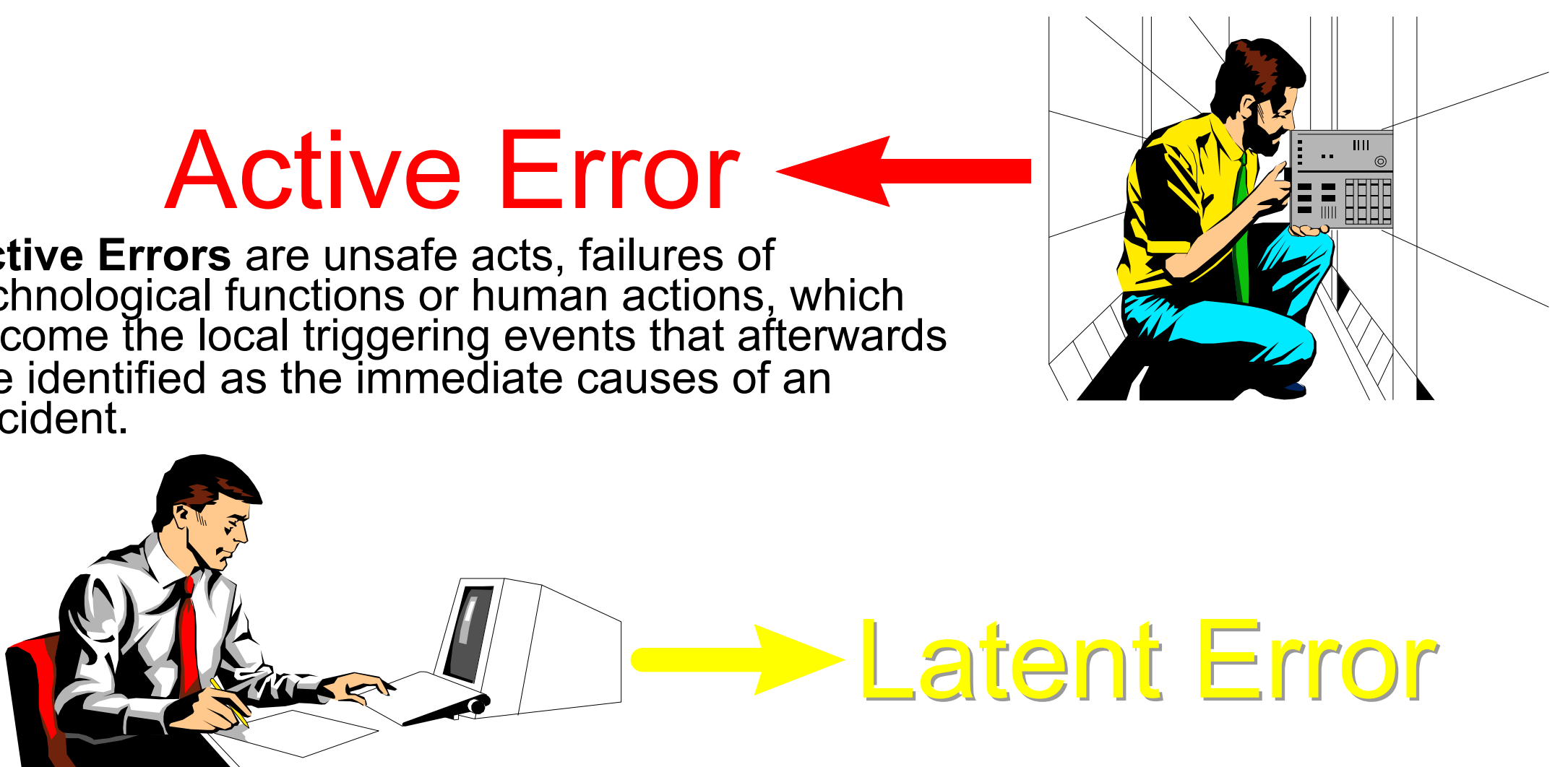

Latent Errors result in latent conditions in the system that may become contributing causes for an accident. They are present within the system as unnoticed conditions well before the onset of a recognizable accident sequence. 


\section{We do know that:}

Active Errors + Latent Errors + Unique Situations

lead to ACCIDENTS! 


\section{Latent and Active Error Frequencies from 37 Operating Events (NUREG/CR-6753, 2002)}

\begin{tabular}{|l|c|c|}
\hline Category (followed by human performance influence) & $\begin{array}{c}\text { Latent } \\
\text { Errors }\end{array}$ & $\begin{array}{c}\text { Active } \\
\text { Errors }\end{array}$ \\
\hline Operations & & \\
\hline $\begin{array}{l}\text { Command and control issues including crew resource } \\
\text { management, }\end{array}$ & 4 & 14 \\
\hline Failure to follow safe practices & 1 & \\
\hline Inadequate knowledge or training & 12 & 2 \\
\hline Incorrect operator actions & 3 & 7 \\
\hline Communications & 3 & 2 \\
\hline Design and Design Change Work Process & & \\
\hline Design deficiencies & 19 & \\
\hline Design change testing & 5 & \\
\hline Inadequate engineering evaluation & 8 & \\
\hline Ineffective indications for abnormal condition & 1 & \\
\hline Configuration management & 6 & 1 \\
\hline
\end{tabular}




\section{Latent and Active Error Frequencies (cont.)}

\begin{tabular}{|l|c|c|}
\hline Category (followed by human performance influence) & $\begin{array}{c}\text { Latent } \\
\text { Errors }\end{array}$ & $\begin{array}{c}\text { Active } \\
\text { Errors }\end{array}$ \\
\hline Maintenance Work Process & & \\
\hline Poor work package preparation, QA and use & 7 & \\
\hline Inadequate maintenance practices & 17 & \\
\hline Inadequate technical knowledge & 4 & \\
\hline Inadequate post-maintenance Testing & 9 & \\
\hline Procedural Design and Development Process & & \\
\hline Inadequate procedures & 18 & 1 \\
\hline $\begin{array}{l}\text { Organizational Learning and Corrective Action } \\
\text { Program }\end{array}$ & & \\
\hline Failure to respond to industry and internal notices & 7 & \\
\hline Failure to follow industry operating practices & 2 & \\
\hline Failure to identify by trending and problem reports & 10 & \\
\hline Failure to validate vendor reports & & \\
\hline
\end{tabular}




\section{Latent and Active Error Frequencies (cont.)}

\begin{tabular}{|l|c|c|}
\hline Category (Followed by human performance influence) & $\begin{array}{c}\text { Latent } \\
\text { Errors }\end{array}$ & $\begin{array}{c}\text { Active } \\
\text { Errors }\end{array}$ \\
\hline Work Prioritization & & \\
\hline Failure to correct known deficiencies & 15 & \\
\hline Continue to operate during unstable conditions & 1 & 2 \\
\hline Management Oversight & & \\
\hline Inadequate supervision & 10 & 5 \\
\hline $\begin{array}{l}\text { Inadequate knowledge of plant systems and plant } \\
\text { requirements }\end{array}$ & 2 & 1 \\
\hline Organizational structure & 1 & \\
\hline
\end{tabular}




\section{Exercise: Medical Misadministration}

\section{Read the two medical misadministration}

examples in Appendix B

- Identify the errors that were committed

-What caused the errors?

- How might these errors be prevented in the future? 


\section{Who's at Fault?}

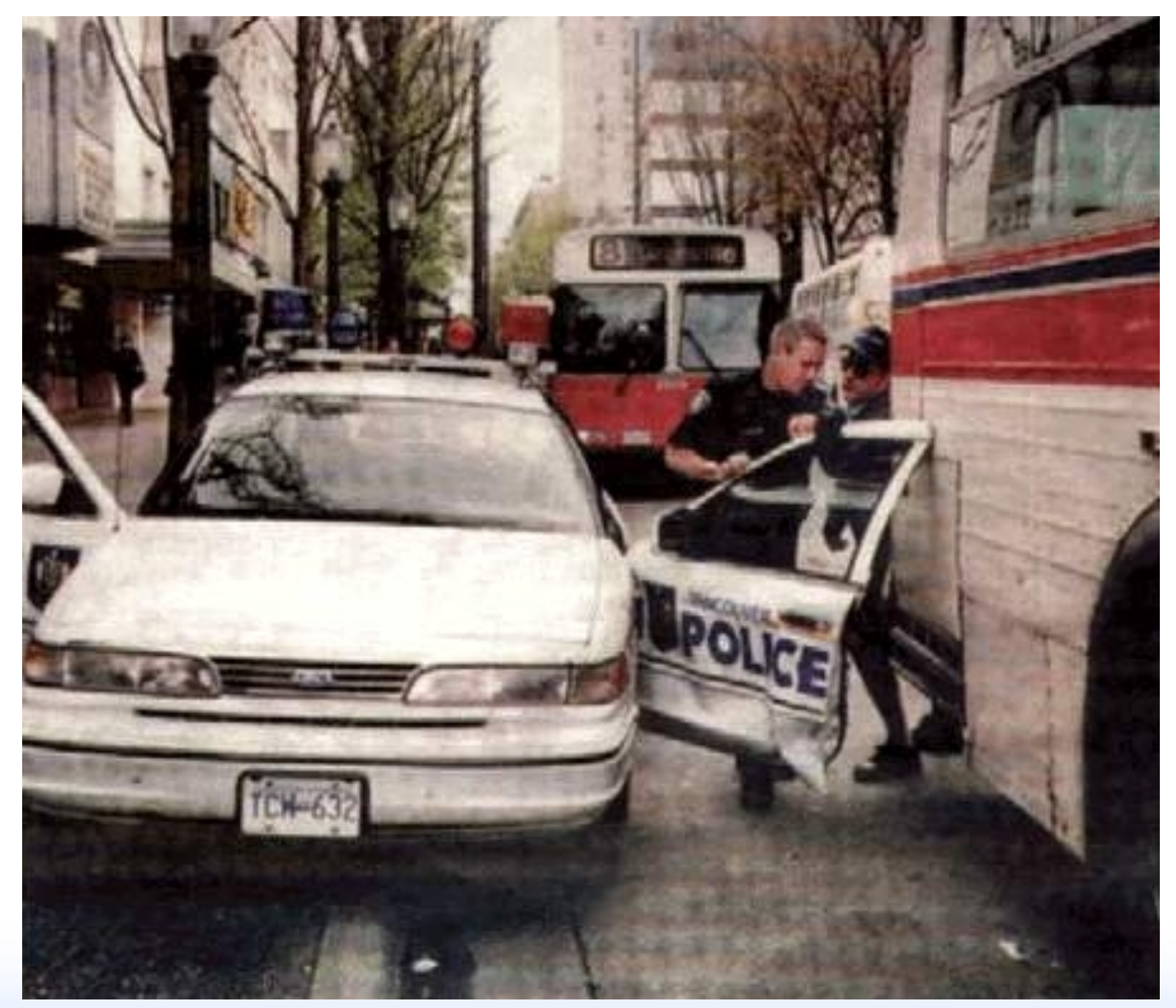

Idaho National Laboratory 


\section{Old and New Views of Human Error}

Sidney Dekker in The Field Guide to Understanding Human Error (2006) suggests that the concept of "human error" may be misleading

The Old View of Human Error: The "Bad Apple" Theory

- Humans are unreliable

- Human errors cause accidents

- Failures come as unpleasant surprises

The New View of Human Error

- Human error is the effect or symptom of deeper trouble

- Human error is systematically connected to people's tools, tasks, and operating environment

- Human error is not the conclusion of an investigation but rather the starting point 


\section{Old and New Views of Human Error (cont.)}

Dekker suggests that the "old view" oversimplifies

- Somebody didn't pay enough attention

- If only somebody had caught the error, then nothing would have happened

- Somebody should have put in a little more effort

- Somebody thought that taking a safety shortcut was not such a big deal

The "new view" tries to capture the complexity of the situation

- Safety is never the only goal of a worker

- People do their best to reconcile goals and make trade-offs (efficiency vs. safety)

- Nobody comes to work to do a bad job!

- A system isn't automatically safe unless safety is created in the organization-this is the safety culture of the organization

- New tools and technologies introduce new opportunities for errors 


\section{Human Error and Safety Culture}

\section{Chairman Dale E. Klein's Remarks at the Regulatory Information Conference (RIC), March 10, 2009}

Let me touch on a few areas where I think we need to be proactive, rather than passive. The first is safety culture. Let me be clear in saying that the safety record of the nuclear power industry in the U.S. is on the whole very impressive. And despite some problems, there have been measurable, industry-wide improvements in safety. ...But let's not kid ourselves into thinking that everything is fine. We have continued to see incidents over the last few years that indicate that safety culture was not a priority throughout all the staff, at all the plants. In fact, even an excellent plant can have problems because-paradoxically_excellence can have its own risks. An excellent record can sometimes invite complacency, and make it hard to manage expectations. ... One way to combat complacency is to have a clear plan for promoting safety culture. The NRC recognizes that implementing the day-to-day details of safety culture is the responsibility of the licensees. Nevertheless, the agency is taking a more active role.

...Let me emphasize...that we are not doing this to point fingers... Overall, I think while both the NRC and industry have a strong foundation, there is room for improvement. And there are still things I see here and there that resemble complacency. One way to help avoid complacency is through communication and sharing knowledge. 


\section{Concluding Thoughts on Human Error}

\section{Some Lessons Learned}

- Human errors are frequent and significant contributors to accidents and events

- Latent errors contribute as much or more to accidents as do active errors

- Human error is not about blaming individuals; it's about understanding the situation that led to the error

- In the remainder of this course, you will learn some of the nuances of identifying, modeling, and quantifying human error and its context 


\section{LESSON 3}

\section{HRA Identification Phase}

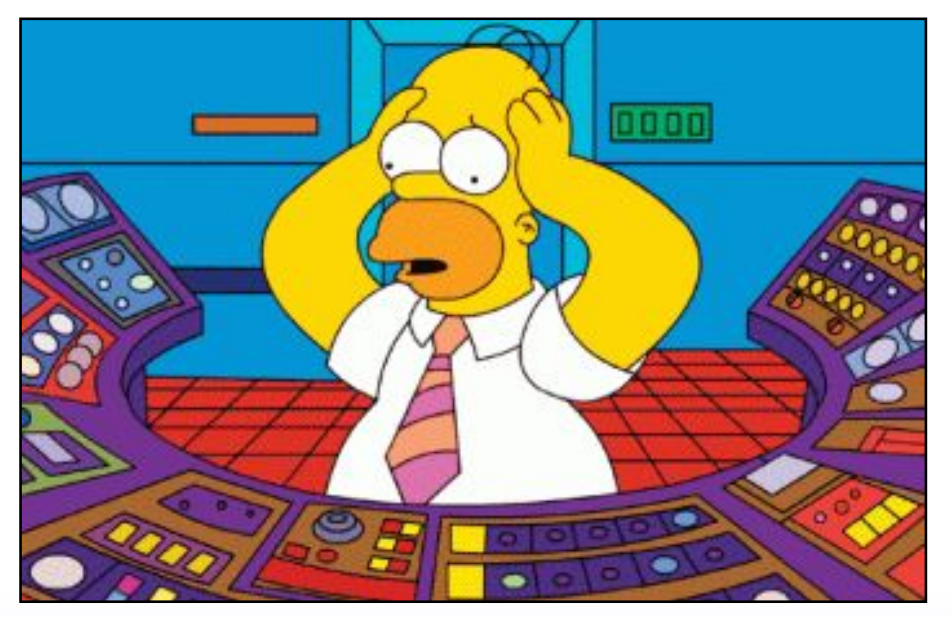




\section{Lesson 3 Objectives}

- Continue discussion of human error, giving specific examples of how human error can be classified for purposes of incorporating into an HRA

- Introduce performance shaping factors

- Internal and external

- Introduce three error taxonomies

- Swain and Guttman

- Reason

- Rasmussen

- Introduce task analysis

- Introduce human error in context of initiating events 


\section{Requirements for Human Reliability Analysis}

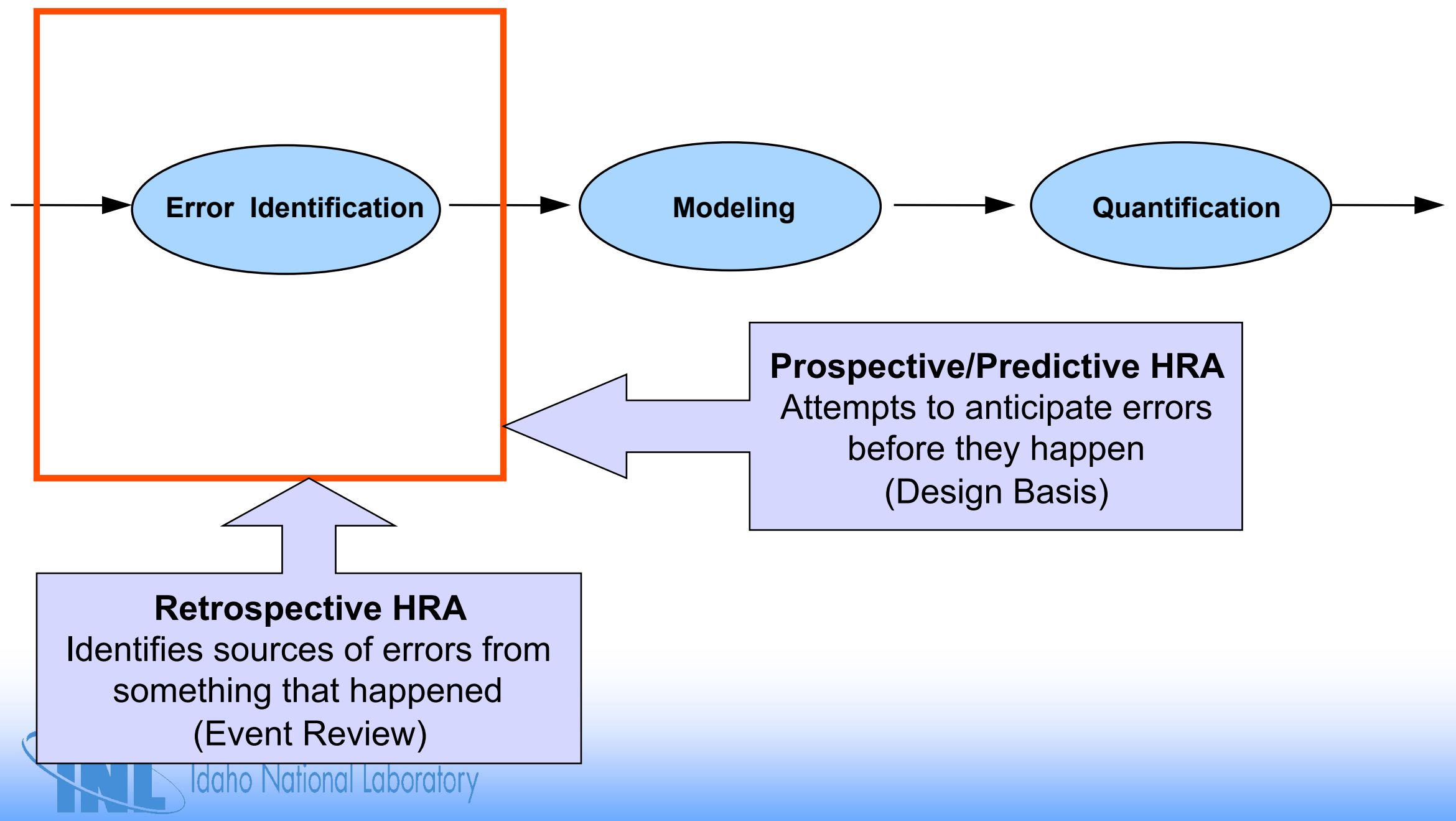




\section{Opportunities for Errors}

Humans are complex systems that must:

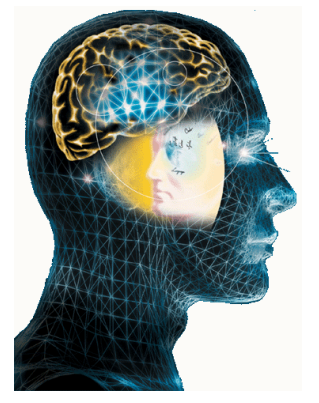

- perceive

- interpret

- decide courses of action

- carry out those actions

Each of these functions present opportunities for errors. 


\section{Human Information Processing}

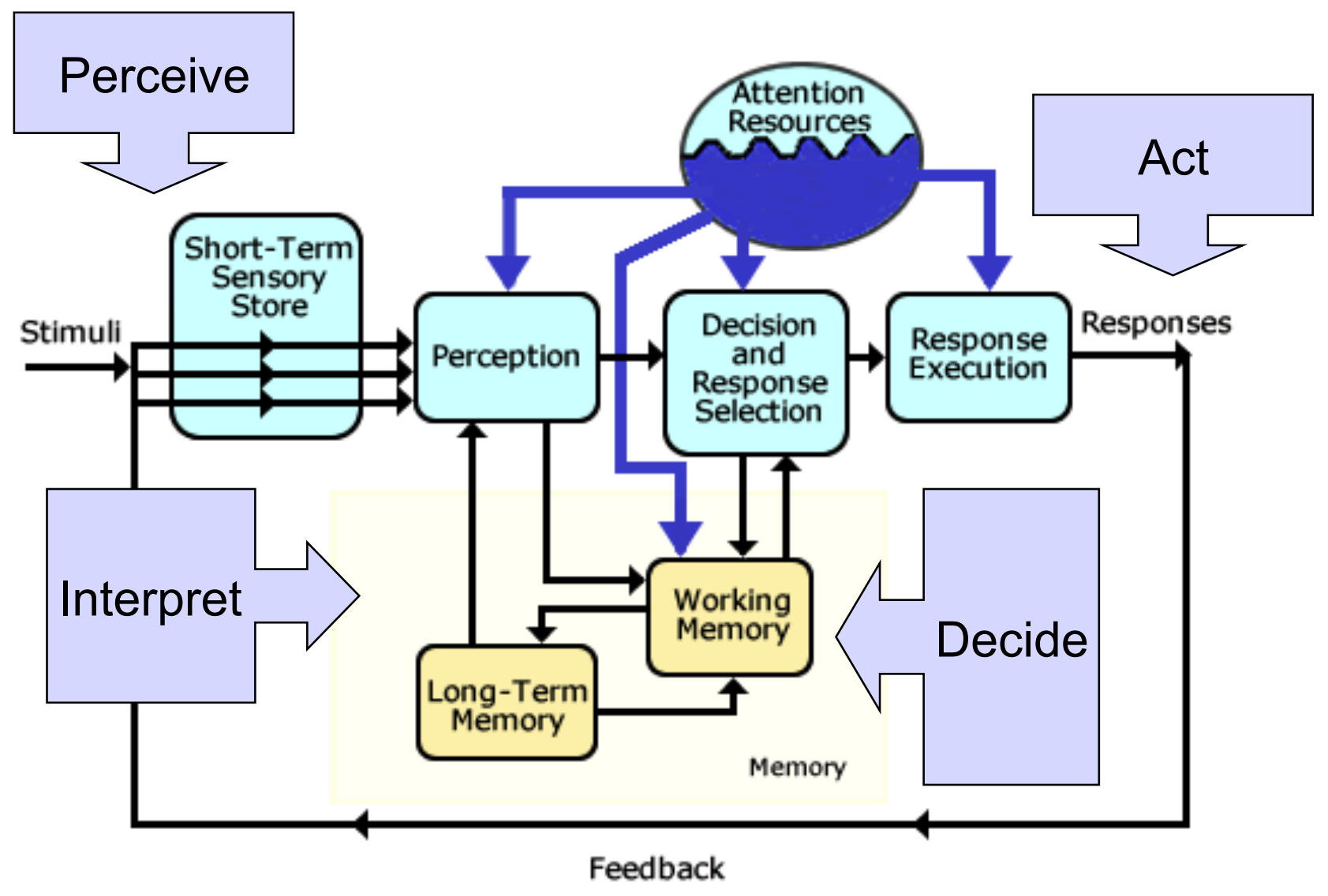

Wickens' Model of Information Processing 


\section{Human-Machine Systems}

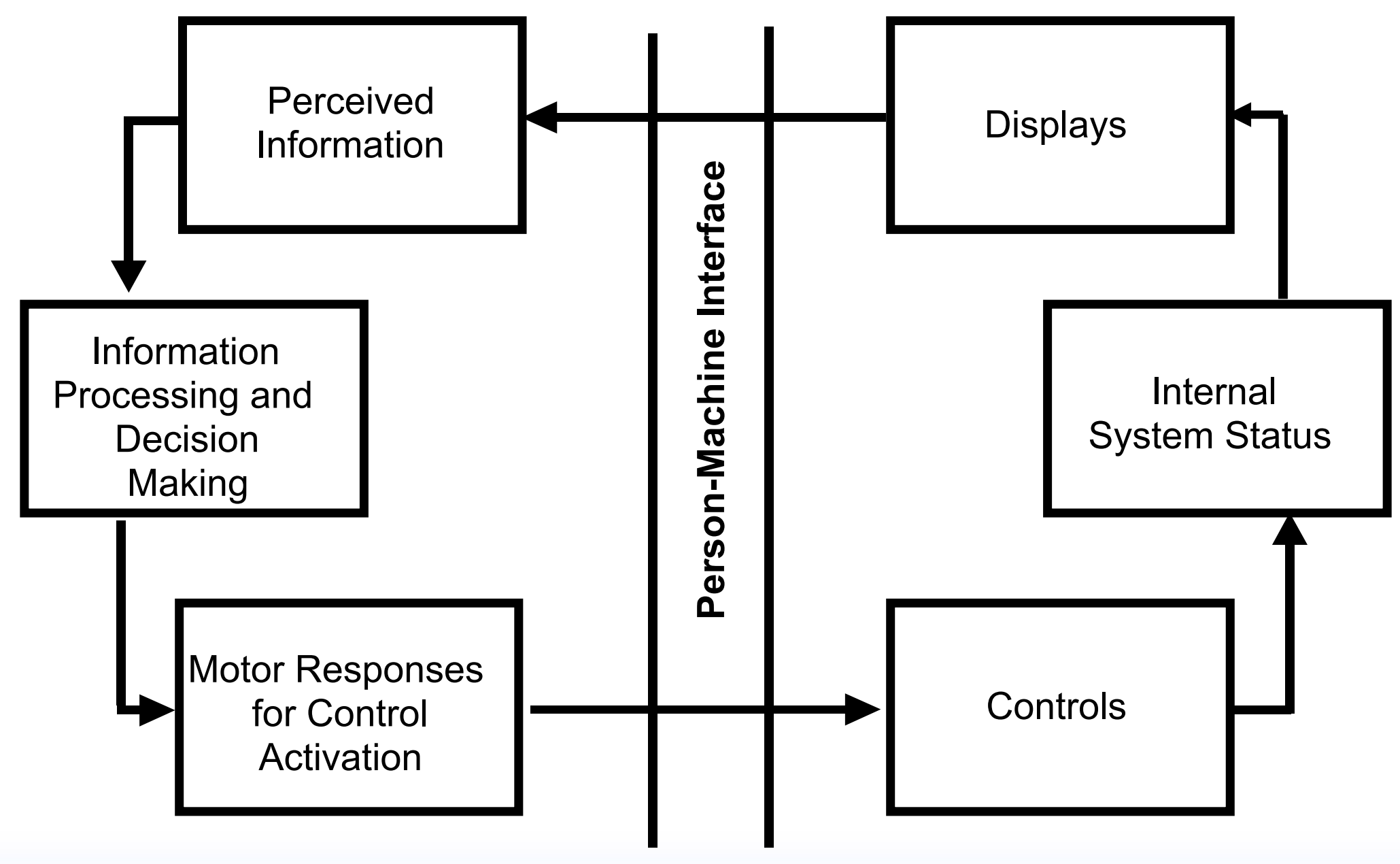




\section{Performance Shaping Factors}

Those factors that influence the performance and error likelihood of the human are called performance shaping factors (PSFs).

PSFs may be internal or external

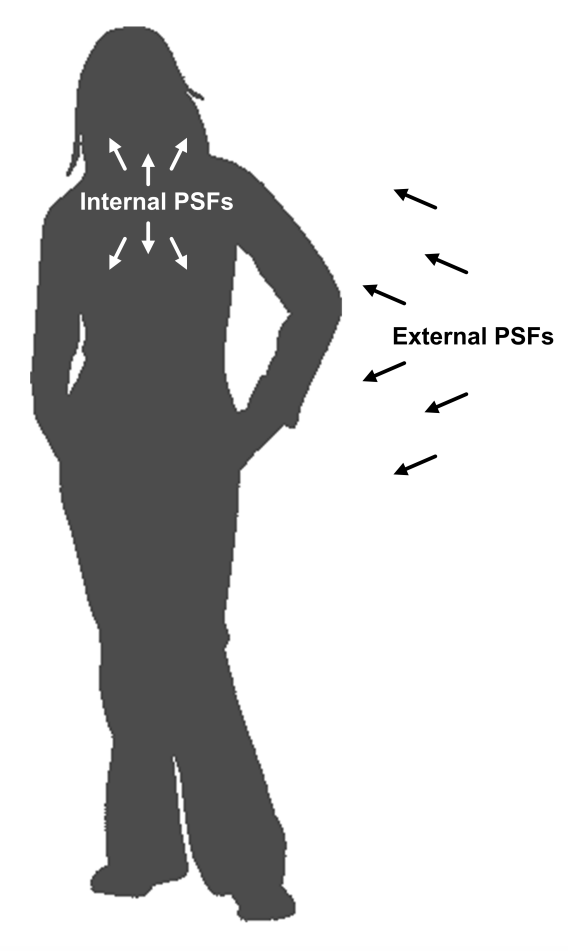




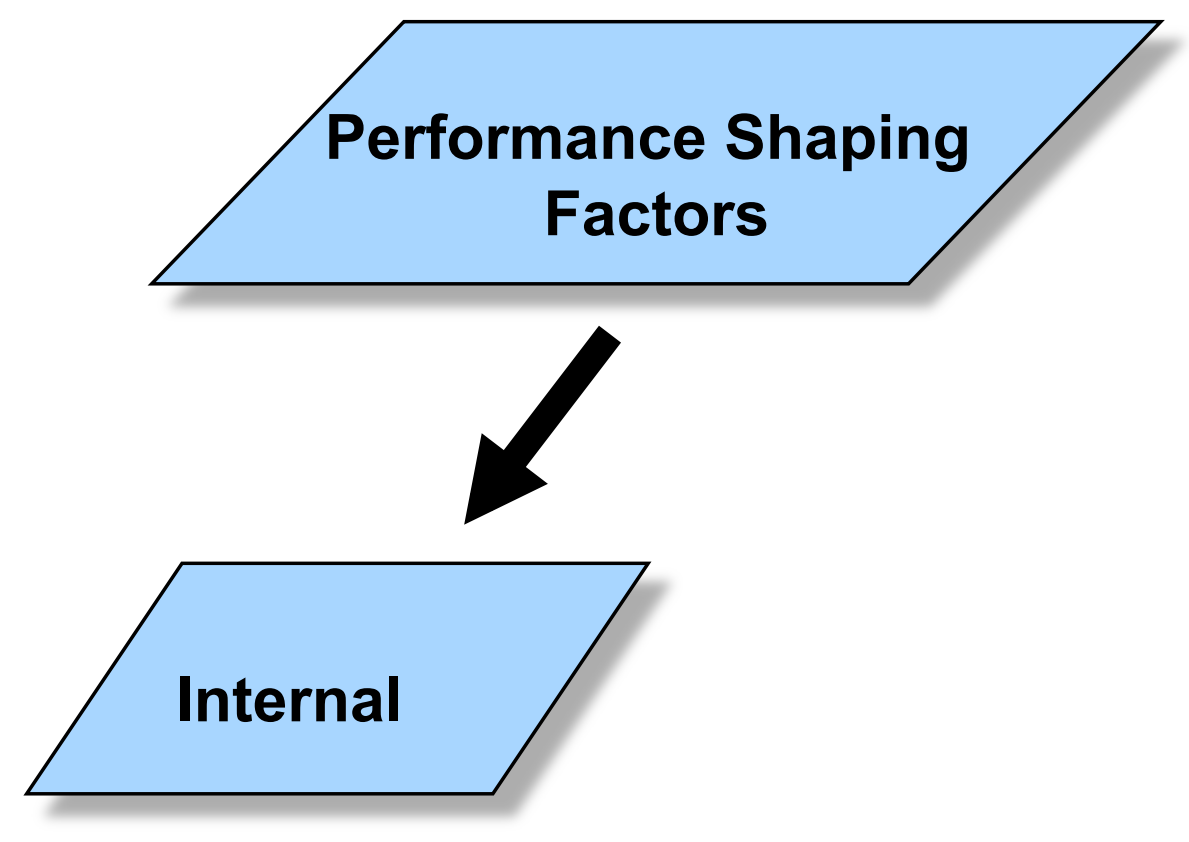

Internal PSFs are human attributes, such as skills, abilities, and attitudes, that operate within the individual, and which are brought to the job by the individual. 
Exercise on human short-term memory ability (or lack thereof): Take out a blank sheet of paper. Listen to the list that the instructor reads to you. When the instructor has finished reading the list, quickly write all the items you can recall on the piece of paper. 


\section{Which Items are Recalled?}

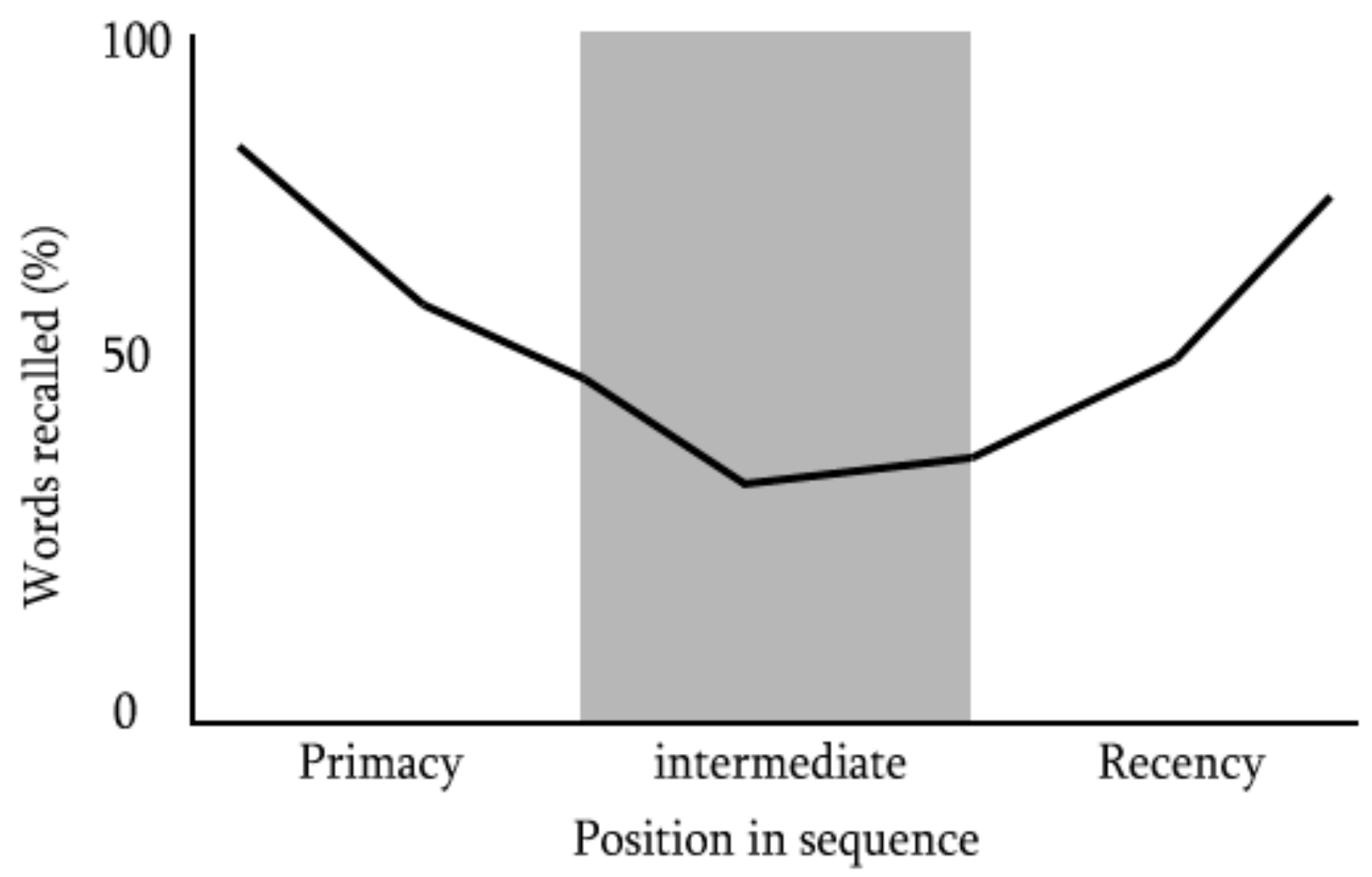




\section{Psychological Context}

\section{Is created by individuals based upon}

- their prior knowledge

- their expectations

- their present circumstances

- their goals

- the reward/punishment structure 


\section{Exercise: Population Stereotypes}

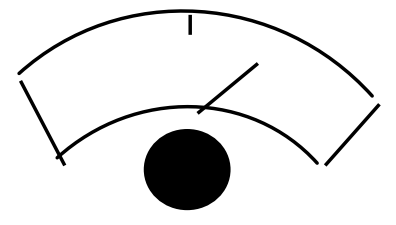

Knob
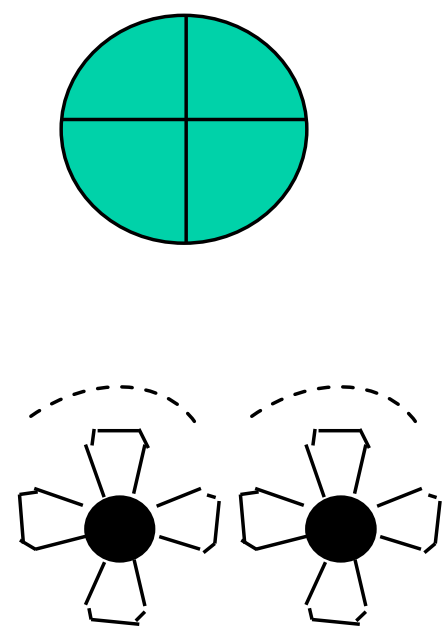

3. Here are 2 knobs on a bathroom sink, looking down at them. Put an arrow on each dotted line, to show how you would use them to turn the water on.

1. To move the arrow-indicator to the center of the display, how would you turn the knob?

\section{clockwise}

counterclockwise

2. In what order would you label the 4 quadrants of a circle. Write in the letters A, B, C, D, assigning one letter to each Quadrant.

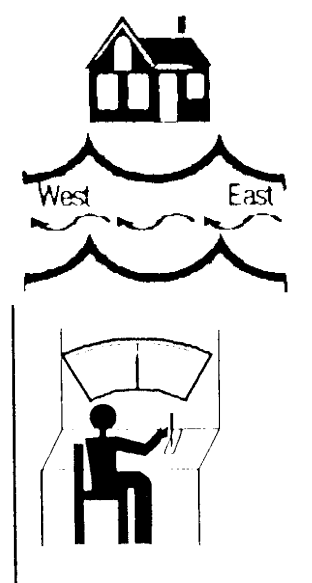

4. Here is a river flowing from east to west. Is the house on the left bank? right bank?

5. To move the arrow indicator to the right of the display, how would you move the lever? Push
Pull

6. Here are two knobs on a bathroom sink, looking down on them. Put an arrow on each dotted line, to show how you would operate them to turn water on.

7. To increase the number in the displayed window, how would you turn the knob?

\section{clockwise} counterclockwise 


\section{Example: Stress as an Internal PSF}

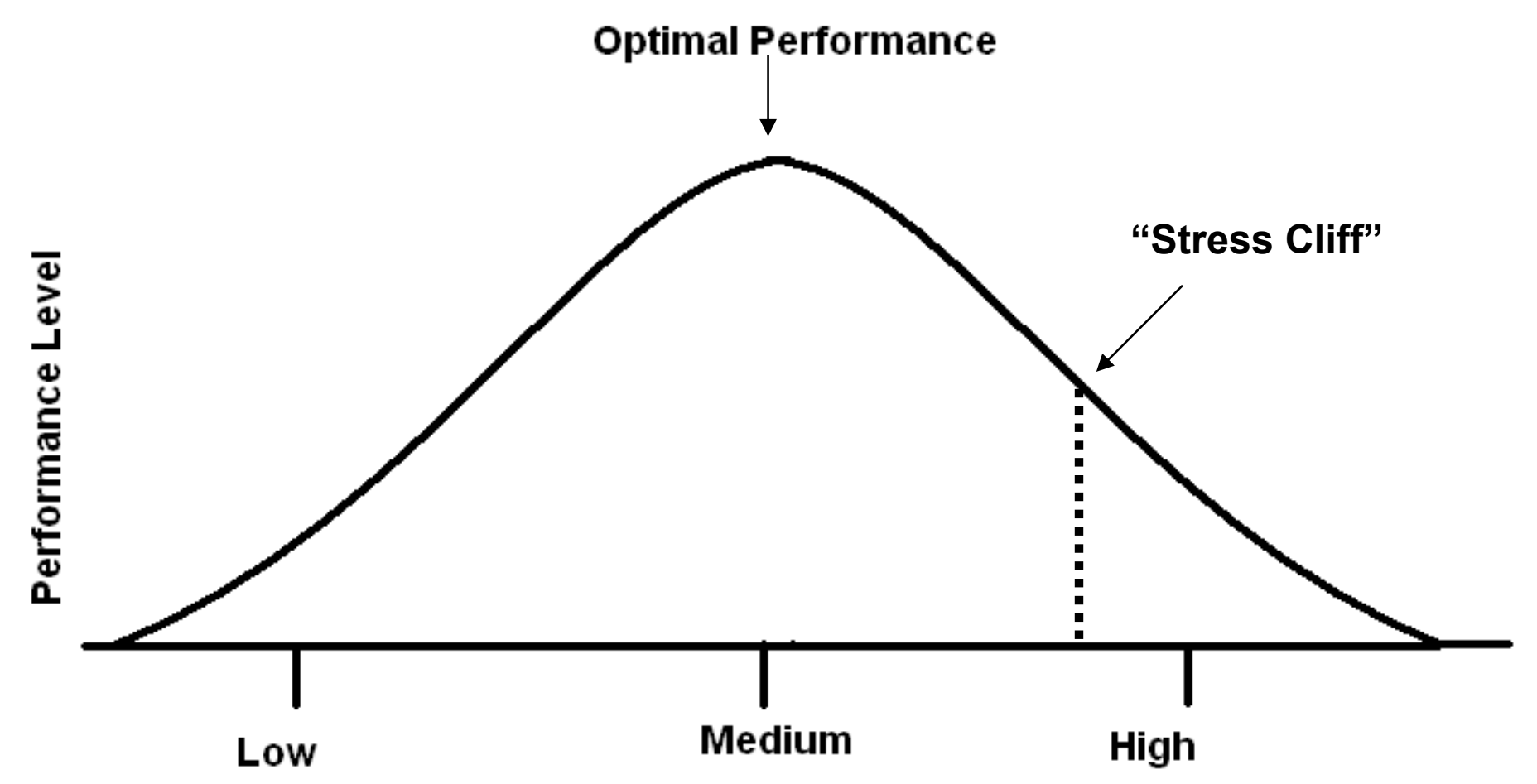

Arousal / Stress Level 


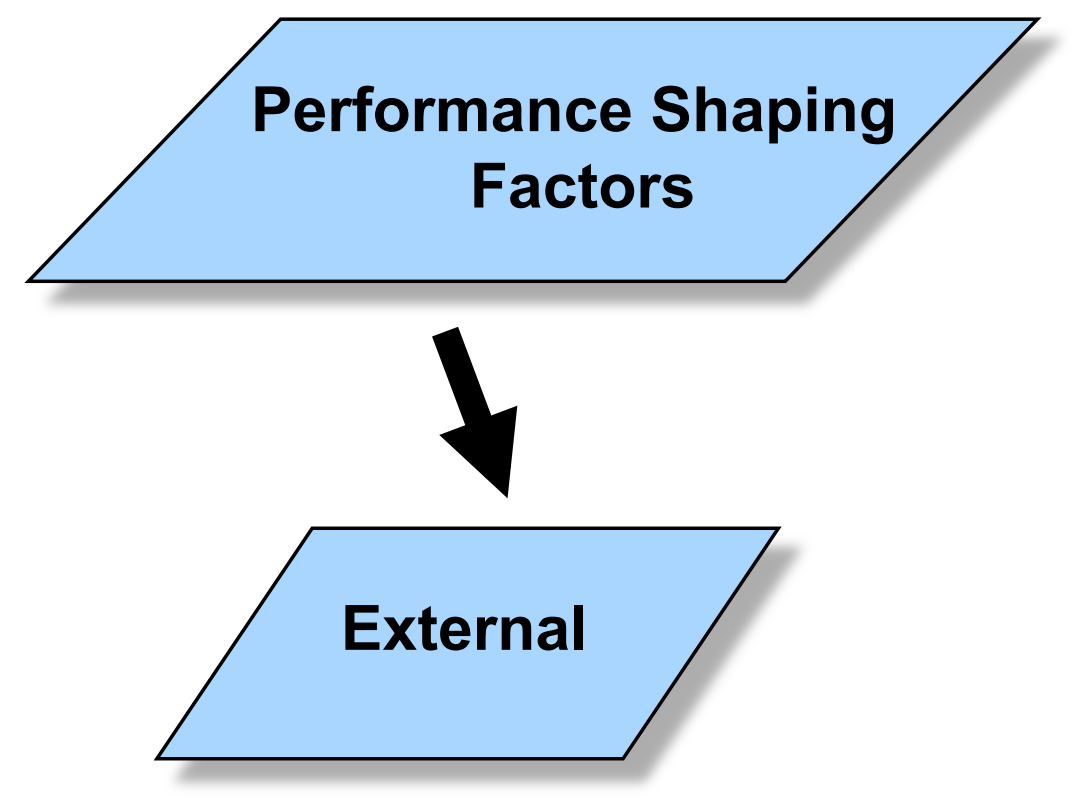

\section{External PSFs are aspects of situations, tasks, and equipment characteristics that influence performance.}




\section{Example: Noise as an External PSF}

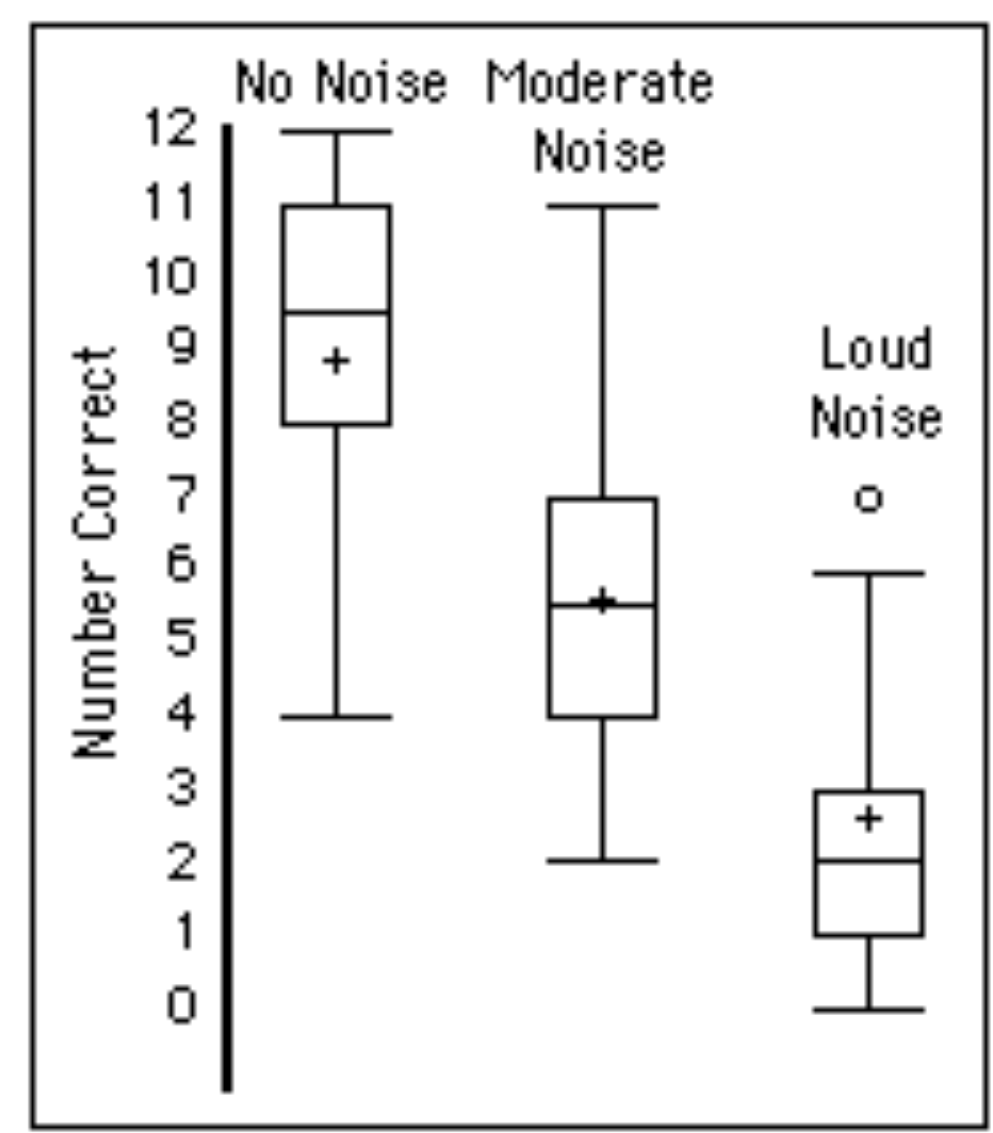

Figure 1. Box plots of the data for the three conditions. 


\section{Example: Ergonomics as an External PSF}
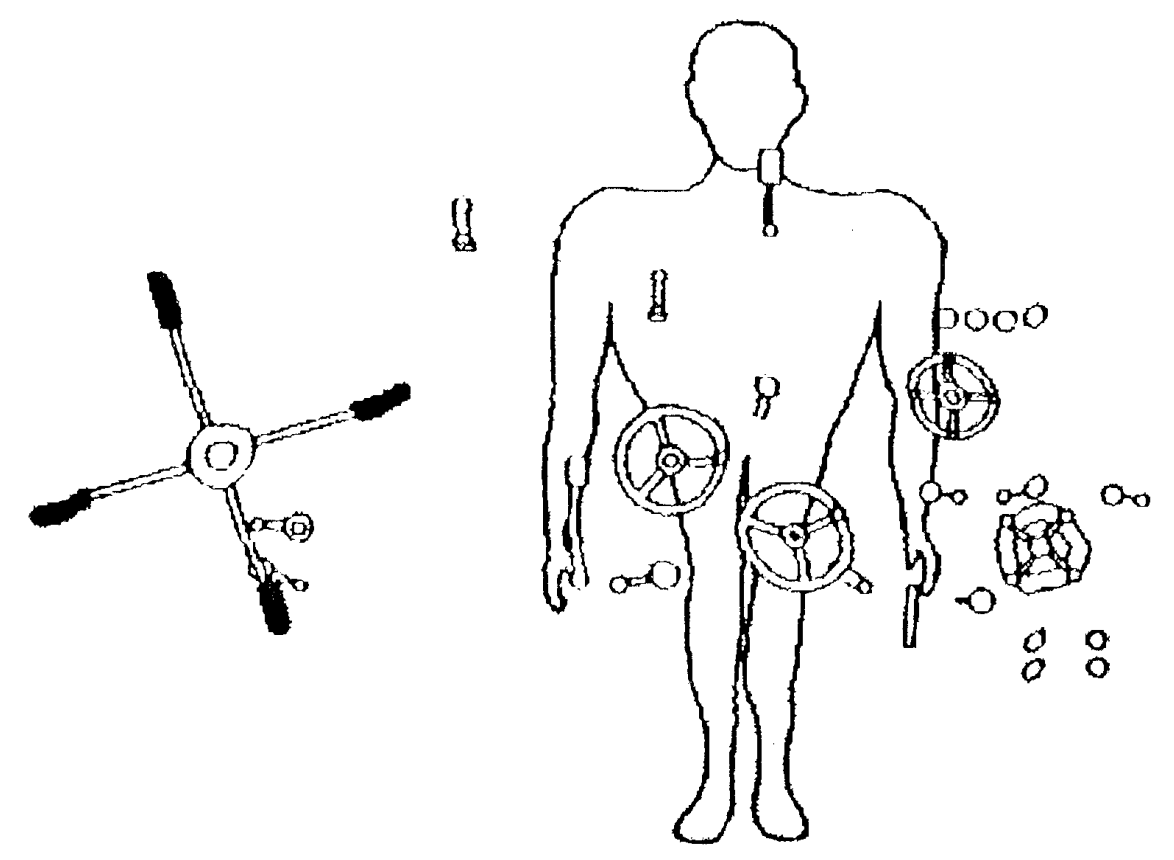

8

The controls of this lathe, in current use, are placed so that the ideal operator should be $4.25 \mathrm{ft}$. tall, $2 \mathrm{ft}$. across the shoulder, and have an $8 \mathrm{ft}$. arm span! 
Exercise: What internal and external PSFs do you think may have been involved in this accident?

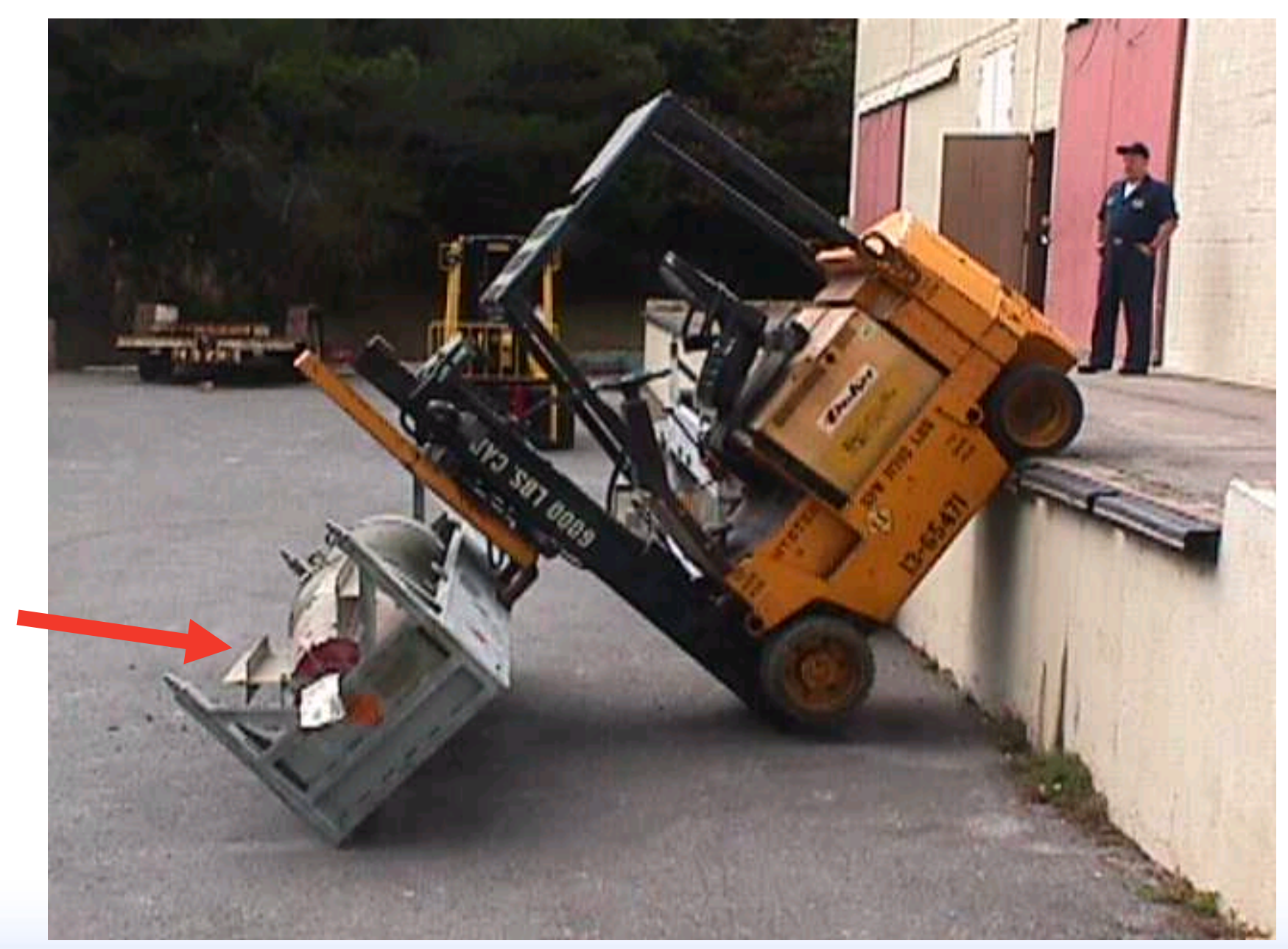




\section{Good Practices PSFs}

\section{NUREG-1792 identifies Good Practices for HRA}

- Also identifies PSFs that should be considered in a quality HRA

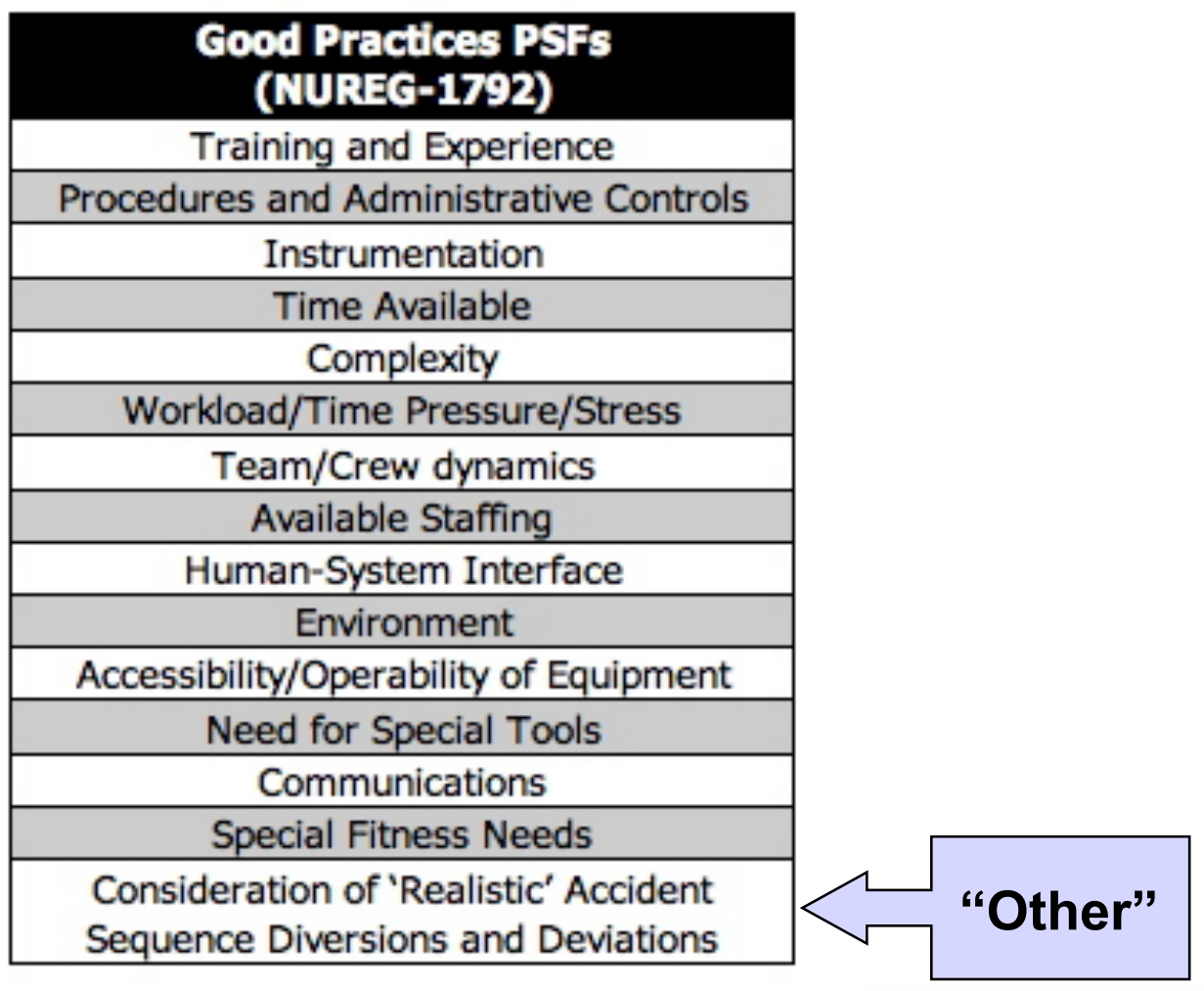




\section{Exercise: PSF Exercise}

1. Divide into groups.

2. Problem definition: List all the performance shaping factors that may influence the reliability of an everyday task like driving to work.

3. For each performance shaping factor, identify and describe the mechanisms of how that factor affects the performance of the task.

4. Describe how you might measure those factors. 


\section{Taxonomies of Human Error}

\section{Taxonomy}

- Systematic grouping according to laws and or principles

- Different HRA methods have different taxonomies

Benefits

- Aids analysts in identifying errors

- Ensures consistency in performance characterizations

- Helps analysts determine the underlying reasons for the error

We will examine three taxonomies:

- Swain and Guttman's Taxonomy (Commission/Omission)

- Rasmussen's Cognitive Taxonomy (Skill/Rule/Knowledge)

- Reason's Error Taxonomy (Slips/Lapses/Mistakes) 


\section{Swain and Guttman's Taxonomy (1983)}

Errors of omission

- Fail to do something required

Errors of commission

- Do something you shouldn't do

Sequence errors

- Do something in wrong order

Timing errors

- Do something too slowly or too quickly 


\section{Rasmussen's Cognitive Taxonomy (1979)}

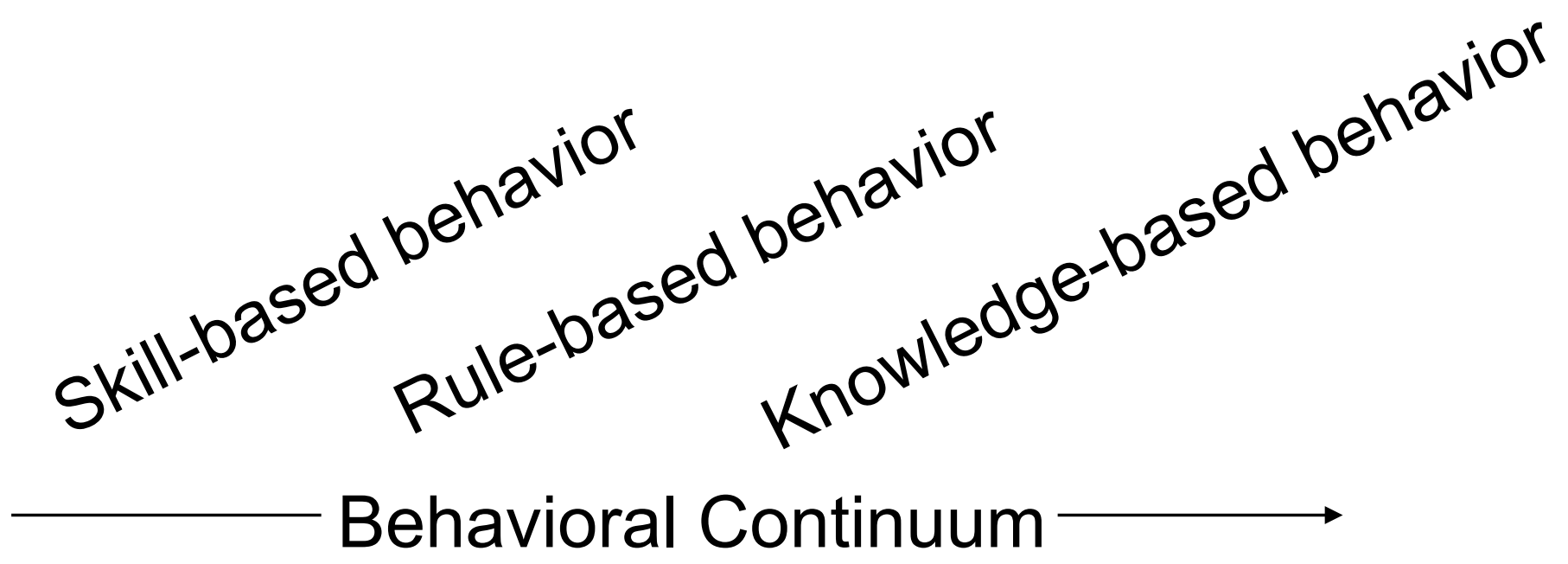

Skill-based $=$ behavior that requires very little or no conscious control to perform or execute an action once an intention is formed (think: highly skilled and automatic)

Rule-based $=$ the use of rules and procedures to select a course of action in a familiar work situation (think: following procedures)

Knowledge-based = type of control that must be employed when the situation is novel and unexpected (think: operators have to rely on problem solving, which requires a lot of resources; they are not old pros at this) 


\section{Performance Modes}

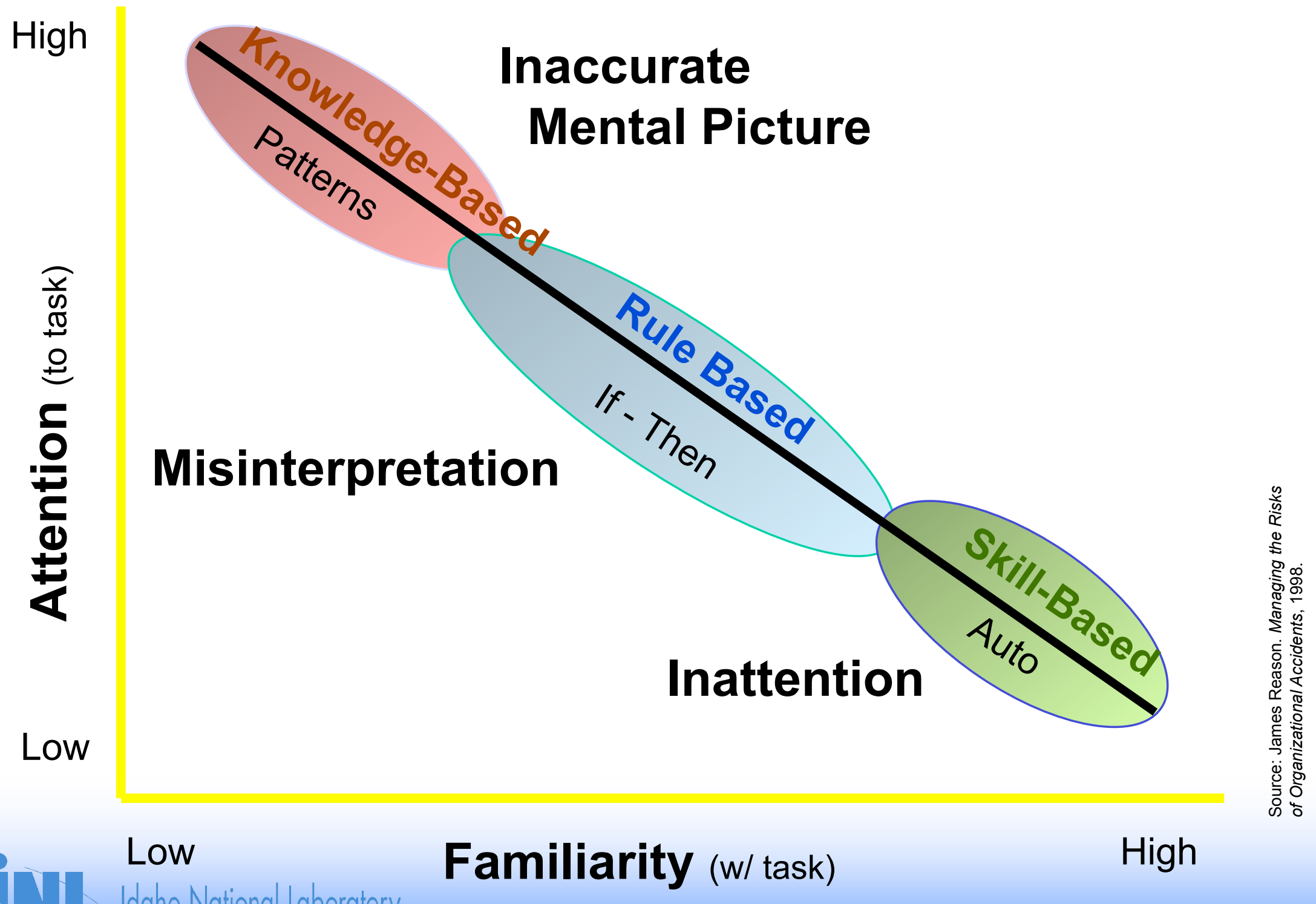




\section{Reason's Error Taxonomy (1980)}

\section{Slips}

- Good intentions, right mental model, but do something wrong

- An error of commission

Lapses

- Good intentions, right mental model, but fail to do something

- An error of omission

Mistakes

- Good intentions, wrong mental model

Violation

- Willful circumvention

- Not necessarily violation in the sense of malevolent intent; can also be "heroism" or "mentality of there's a better way to do something" 


\section{Exercise on Taxonomies}

\section{Select an appropriate classification for each of these errors:}

1. An operator turns off an automated control system.

2. A worker fails to clean out filings after installing a new pipe fitting.

3. A disgruntled electrician reverses two wires on a switch.

4. A painter leaves an emergency diesel generator inoperable after an outage.

5. An operator fails to identify a steamline break immediately due to a missing alarm.

6. A coworker enters a radioactive area without proper protective gear to remove an injured worker.

7. The crew responds incorrectly initially to a plant upset that isn't covered in the procedures.

8. A carpenter lacerates his leg with a circular saw during maintenance activities.

9. Spent fuel personnel do not check to see if the lid is seated properly on a spent fuel canister. 


\section{Case Study: What Happened at Tokai-mura?}

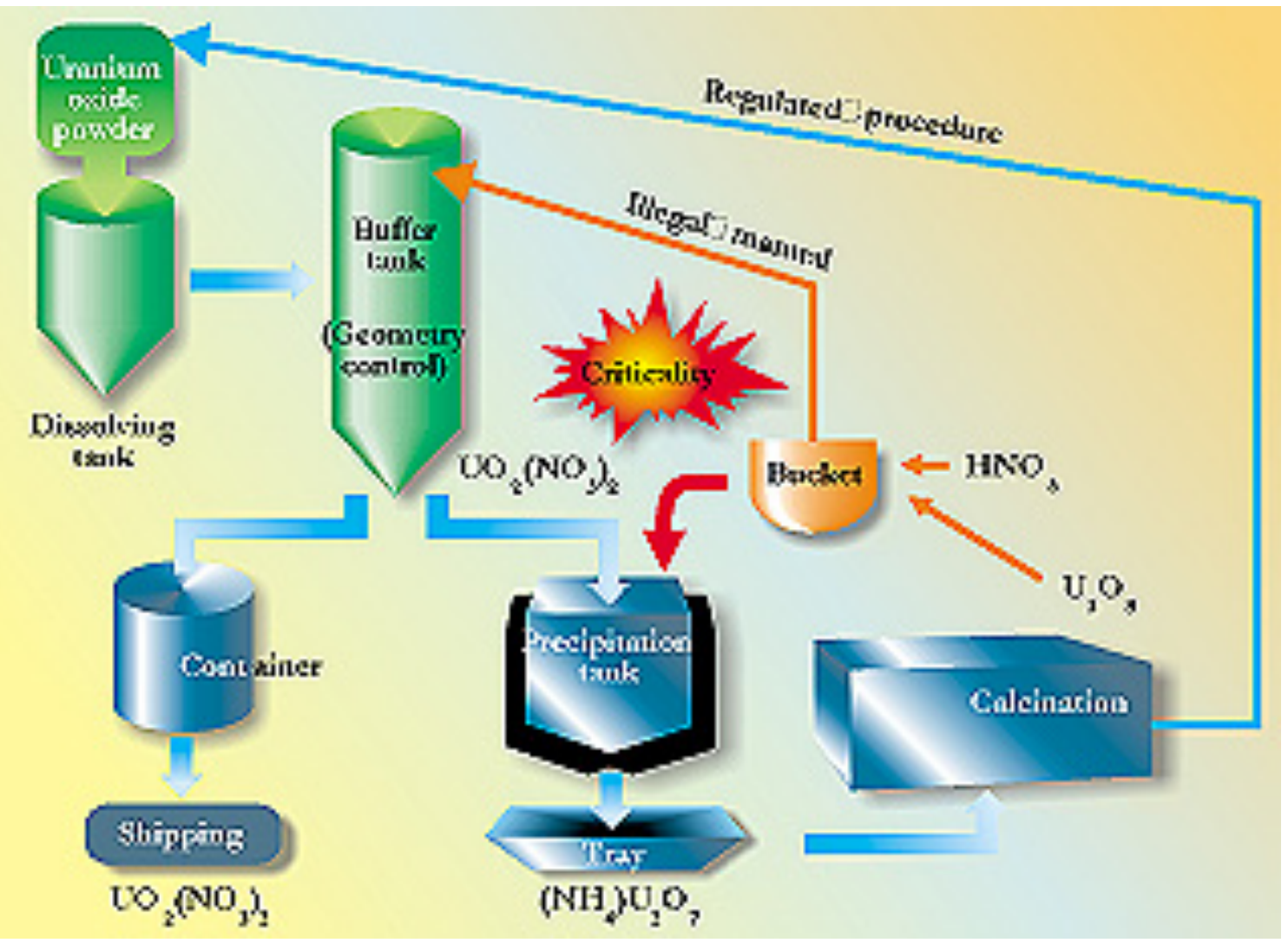

Process flow for the conversion facility at the JCO plant. Blue lines indicate the procedure approved by Japan's Science and Technology Agency. Uranium oxide and nitric acid are fed through a dissolving tank into a buffer tank. Orange lines indicate a company-initiated procedure not approved by STA, in which uranium oxide and nitric acid are added by bucket directly to the buffer tank. The red line indicates buckets dumped directly into a precipitation tank-a further deviation from licensed procedure. 


\section{Exercise: Identifying Human Error}

Divide into teams

Each team will read pp. 182-186 of the Tokai-mura incident in Appendix C (30 min.)

In the discussion, each team will:

- describe human errors

- classify those errors using one of the error taxonomies

- describe any PSFs which they feel may have an impact on the identified errors (30 min.)

Each team will present their work 


\section{Task Analysis}

A technique to help identify human activities in a task

- Think of it as the steps in a procedure of human actions, even though there may be no formal procedure

- May have different levels of task decomposition

- Can model high-level tasks such as everything related under a common task goal (e.g., turn it off)

- Functional system goals are basis of HFE definitions

- Can model low-level tasks such as all activities required (e.g., identify switch, turn switch to off position, verify it is off by disappearance of green "on" light) 


\section{Task Analysis Used to Identify Actions and Decisions}

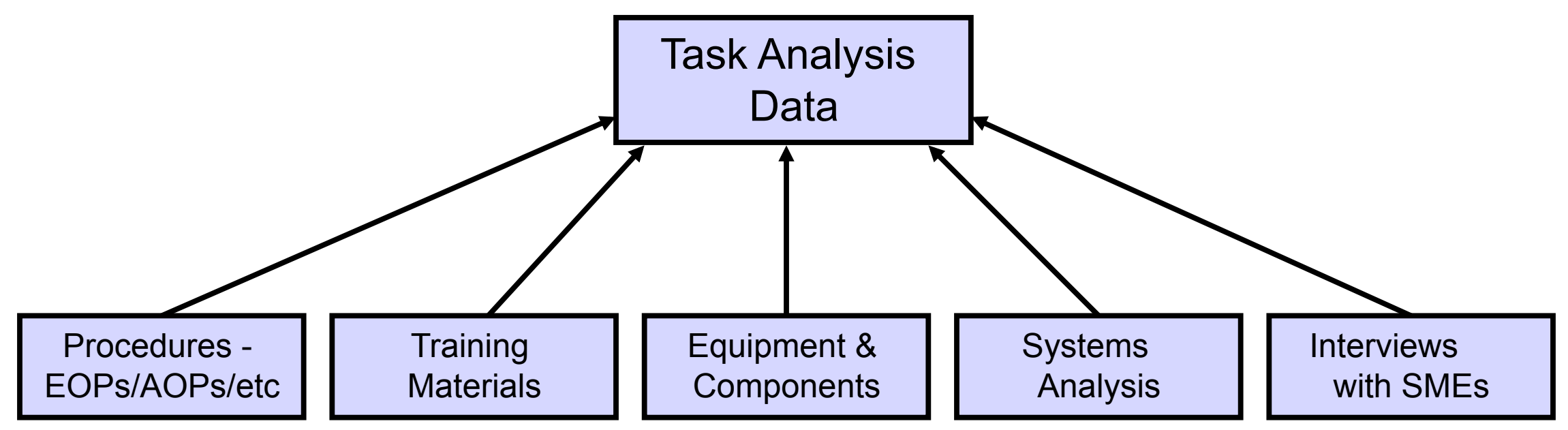

EOP = emergency operating procedure,$A O P=$ abnormal operating procedure, $S M E=$ subject matter expert 


\section{Task Analysis Steps}

- Capture each major decision or decision-action in the sequence of human and hardware activities

- Human actions may be clustered according to a highlevel goal (e.g., "seal cask") with subgoals

- It is useful to treat these as successful or safe human actions vs. unsuccessful or unsafe human actions

- It is often useful to treat these as a chronological sequence of actions

- For event investigation, this would be a timeline

- For prospective risk modeling, this would simply be a consideration of the risk significant activities that take place in plant operations

- Possible or actual human errors are called Human Failure Events (HFEs) 


\section{Task Analysis Exercise}

- Develop a task analysis (i.e., identify the steps/ sequence required) for earlier exercise of "driving to work"

- Hint: think safety-critical functions, performance, etc.

- Identify any new performance shaping factors revealed by this task analysis

- Report out and discuss 


\section{Initiating Events}

Event initiators:

- Help focus the HRA and task analysis

- Human activities are pre-initiator, initiator related, or post-initiator

- Provide sequences and conditions that are generally provided by the Risk Assessment analyst

- Are categorized as:

- Human actions (errors)

- Hardware failures

- Software failures

- External events 


\section{Reactor Initiating Events}

- NUREG/CR-4550, Vol. 1, Rev 1, Jan 1990, pg 3-1, defines initiating events as "those events that disrupt the normal conditions in the plant and lead to the need for reactor subcriticality and decay heat removal."

- NUREG/CR-5750, "Rates of Initiating Events at US Nuclear Power Plants: 1987-1995," Feb 1999, page 6, defines the initial plant fault (read initiating event) as "the first event in a sequence of events caussing or leadding to an unplanned, automatic, or manual reactor trip."

- NUREG/CR-6928, "Industry-Average Performance for Components and Initiating Events at U. S. Commercial Nuclear Power Plants," Feb. 2007. Uses same definition as NUREG/CR-5750 in most cases, but updates frequency estimates.

- Generally speaking: An off-normal event, that left unattended (i.e., no response from operators or automatically actuated systems, would result in an undesired outcome. For nuclear power plants the typical undesired outcome is core damage, but it may also be release of radioactive materials outside the boundaries of the facility (exposure to the public). 


\section{NMSS Example: Initiating Events for Spent-Fuel Pool Risk Analysis}

- Loss of offsite power from plant-centered and gridrelated events

- Loss of offsite power from events initiated by severe weather

- Internal fire

- Loss of pool cooling

- Loss of coolant inventory

- Seismic event

- Cask drop

- Aircraft impact

- Tornado missile 


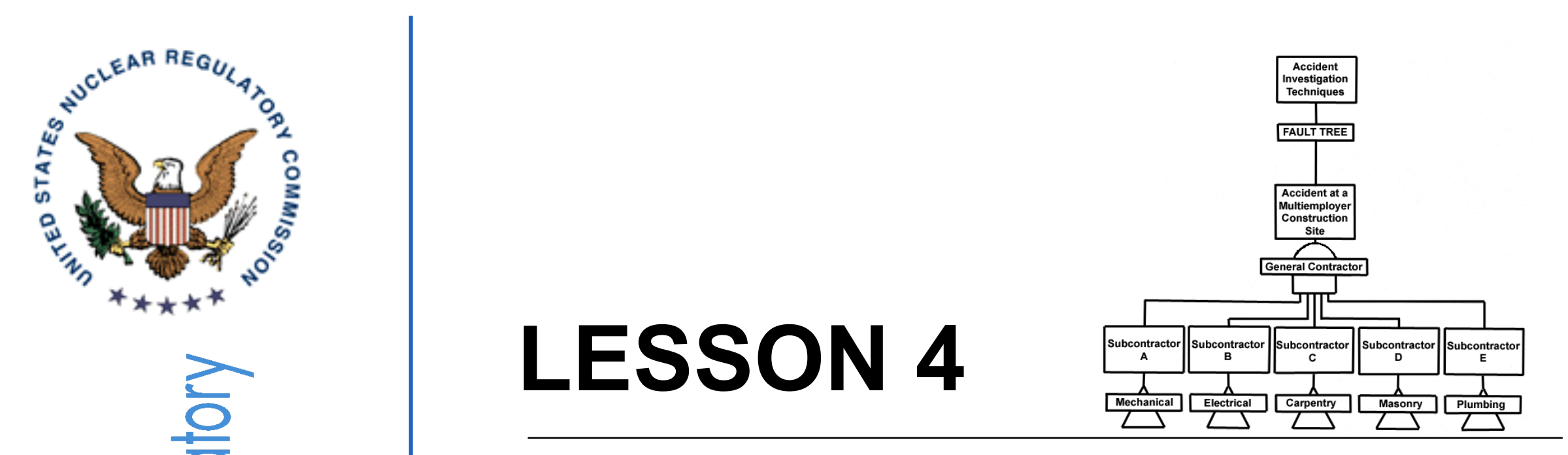

\section{HRA Modeling Phase}




\section{Lesson 4 Objectives}

- Describe how human error can be modeled in PRA

- Review human failure events (HFEs)

- Describe HRA event and fault tree modeling

- Introduce SHARP1 and IEEE 1082 for integrating HRA into PRA 


\section{The essential HRA processes that are integrated into PRAs - HRA Modeling}

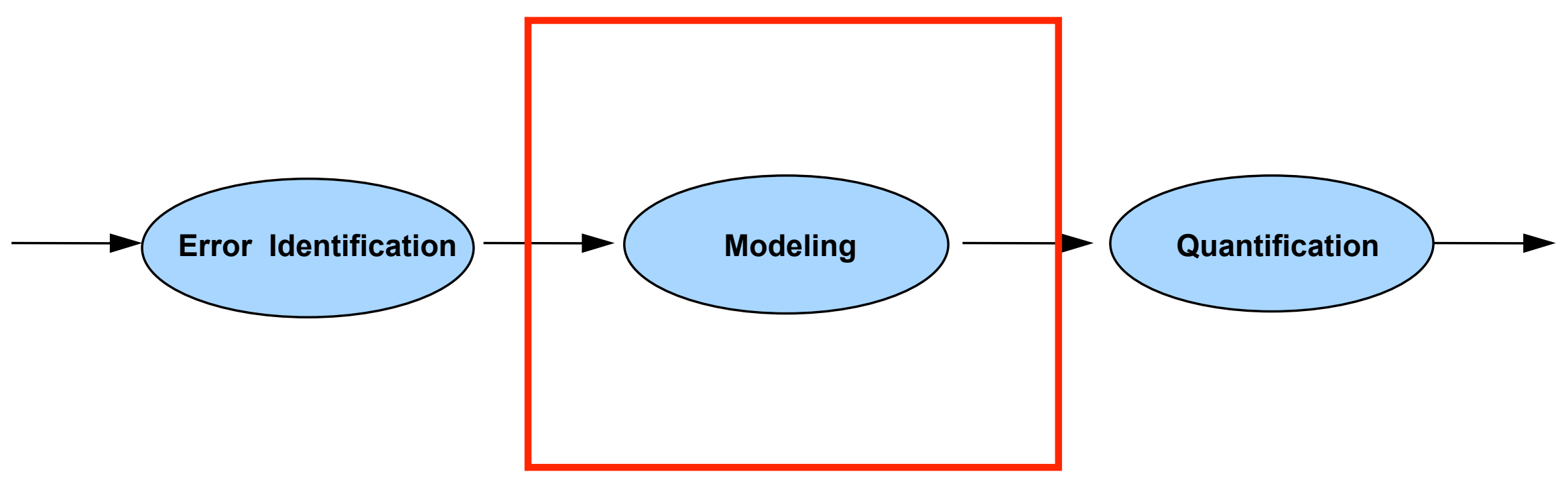




\section{Modeling Human Actions}

What comes after error identification?

- The human activities identified in the first phase of HRA are then modeled or incorporated in the PRA

Recall, that a Human Failure Event (HFE) is:

- A basic event that is modeled in the logic models of a PRA (event and fault trees) and that represents a failure or unavailability of a component, system, or function that is caused by human inaction or inappropriate human action 


\section{Tying HRA Models to System PRA Event and Fault Trees}

Basic modeling in PRA event and fault trees may be:

- At sufficiently low level for quantification (no further analysis is necessary)

- At a high level

If conservative screening values (e.g., human error probability $=0.5$ ) applied to fault trees indicate that a high level human event impacts the overall analysis, there is good justification to perform more detailed modeling and quantification 


\section{Generic System Fault Tree Example}

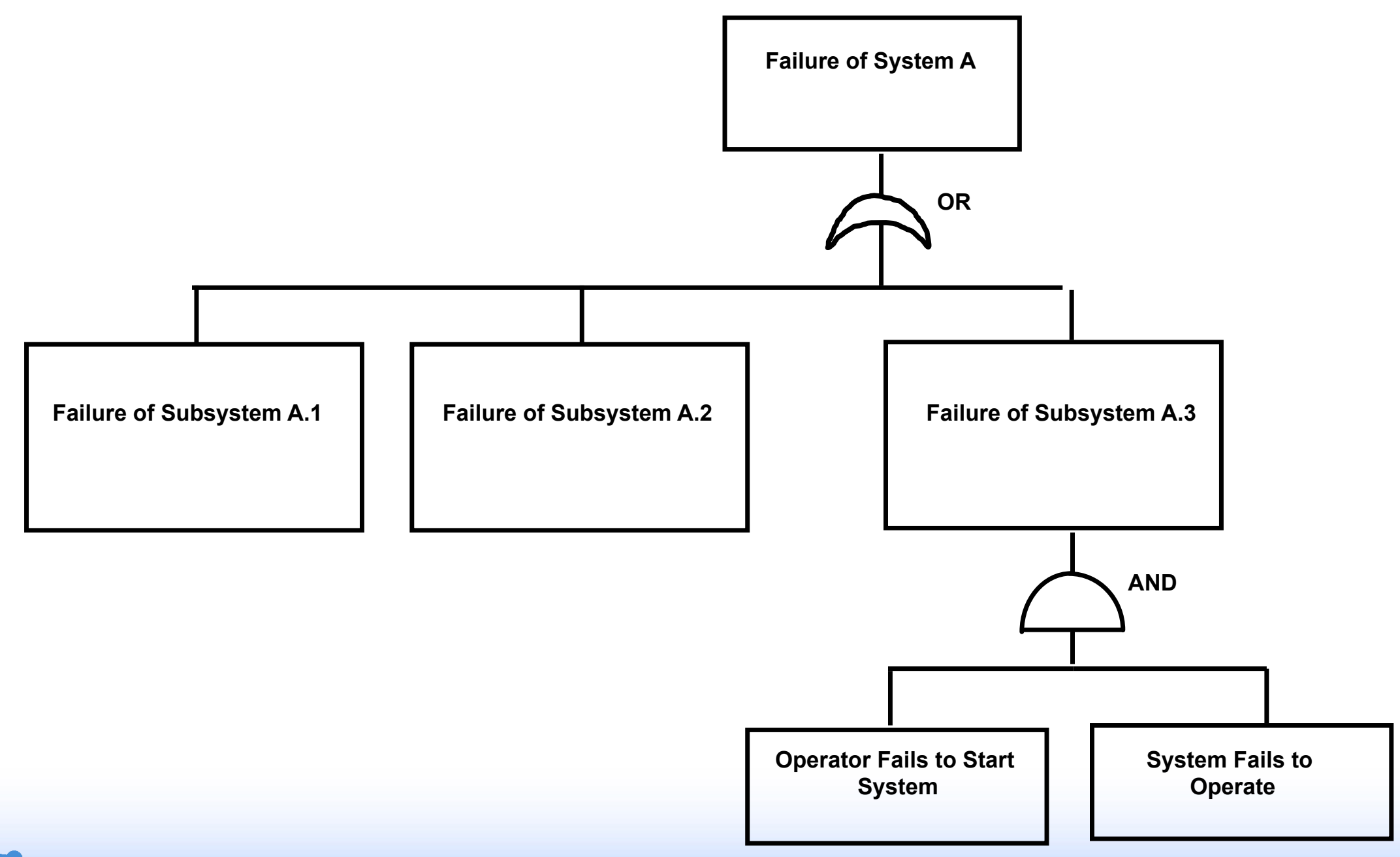




\section{System/Operator Event Tree}

High-level basic human action/event

further analysis may be important

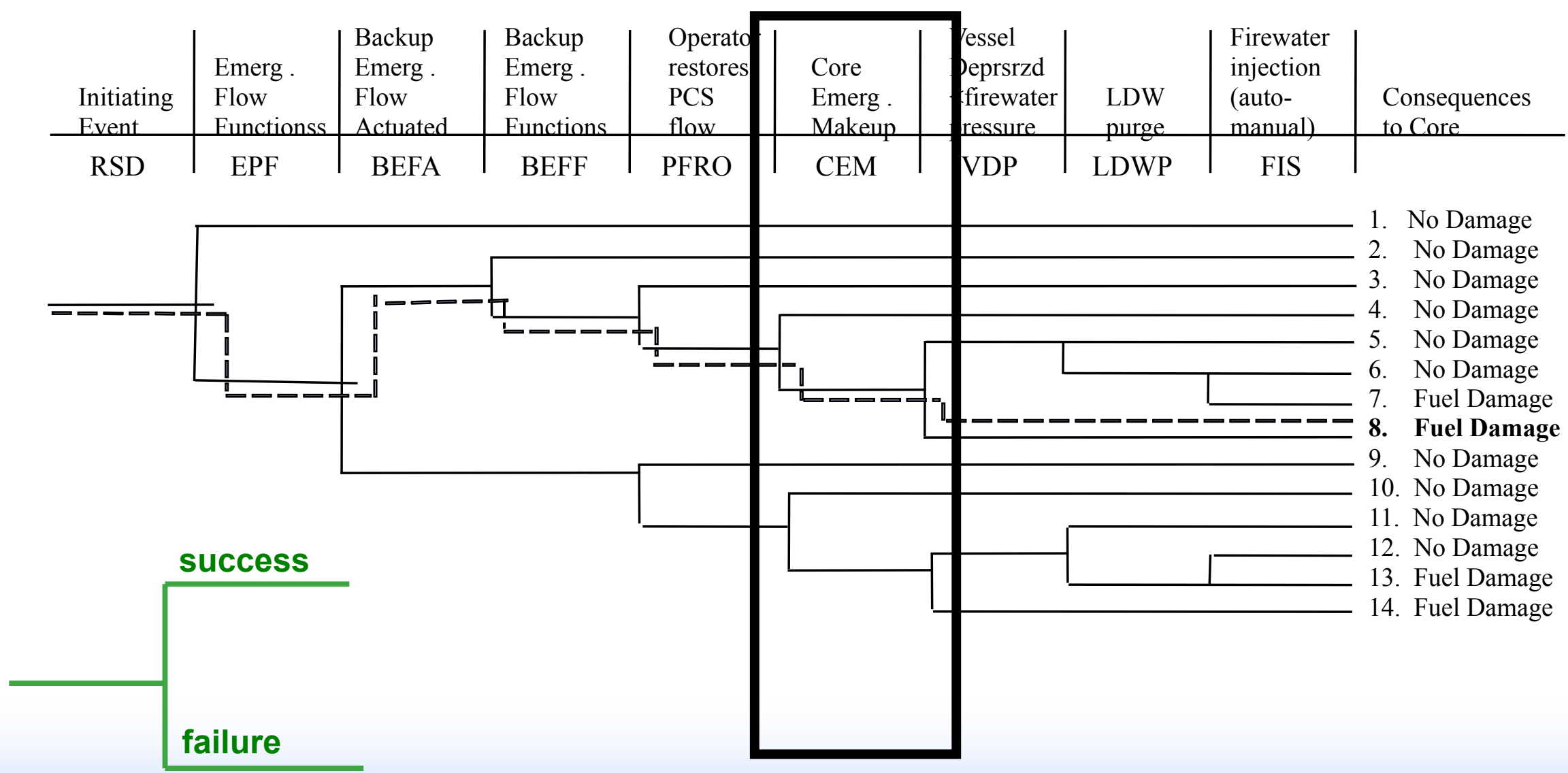




\section{Standard HRA Modeling Techniques}

- HRA Event Trees

- Fault Tree

- Other techniques more applicable to qualitative analyses:

- Influence Diagrams

- Event Sequence Diagrams 


\section{Types of Elements Modeled}

- Correctly performed activities (success)

- Activities leading to failure

- Sequences of failures and successes

- Recovery actions that catch and fix errors before they lead to failure 


\section{Recovery Actions}

- In PRA, recovery may refer to functional recovery

- In HRA, recovery actions are those actions taken by equipment or humans that correct a failure of another action.

- Second Checker

- Alarms

- Automatic Safety Systems 


\section{Bounding and Assumptions}

- The context and assumptions affecting the modeling should be stated explicitly

- Bounding is always needed-impossible to include it all

- How much detail is desirable? (Relates to purpose of analysis or phase--screening vs. realistic)

- What events, steps, and failures should be included? 


\section{HRA Event Tree}

- Developed by Swain and colleagues at Sandia

- Documented in Technique for Human Error Rate Prediction (THERP: NUREG/CR-1278)

- No longer widely used (PRA event and fault trees used more frequently), but has uses:

- Captures recovery information well

- Allows clear delineation of probability of success and probability of failure/error

- Shows sequence of actions better than fault trees 


\section{Sample HRA Event Tree}

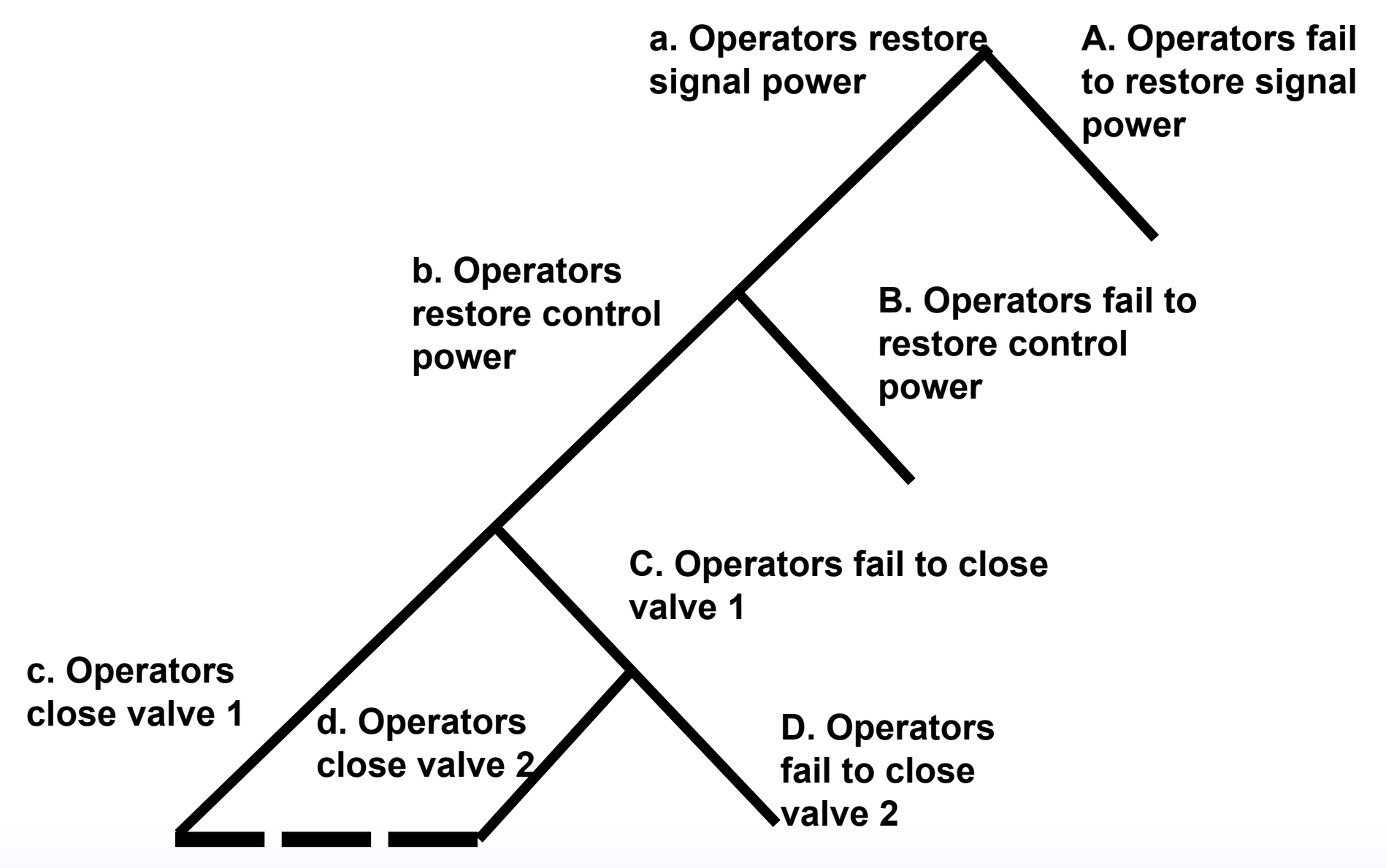




\section{An HRA event tree consists of one or more binary branches (correct/incorrect actions)}

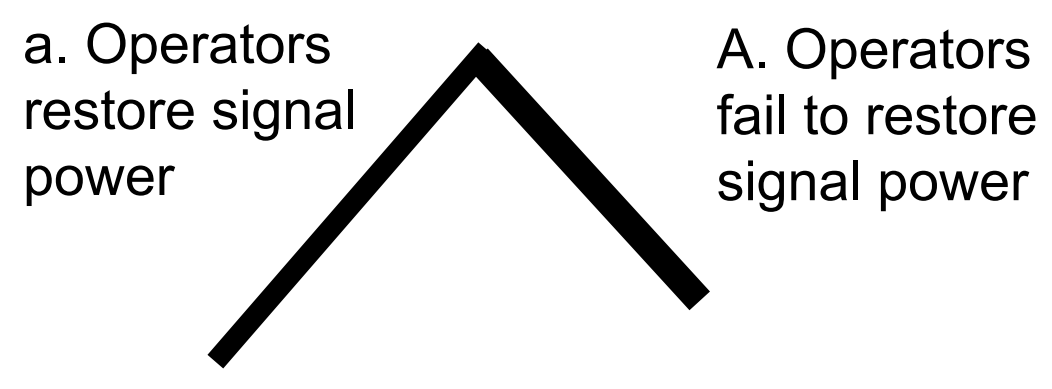


Left

branches

show

successful

actions

Use small

letters for

success

branches

\section{a. Operators} restore signal power

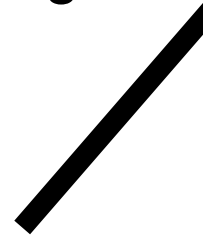

A. Operators fail to restore signal power
Right branches

show

failed

actions

Use

CAPITAL

letters for

failure

branches 
Success branch descriptions are often omitted from tree diagram as they are always the successful complement of the failure statements!

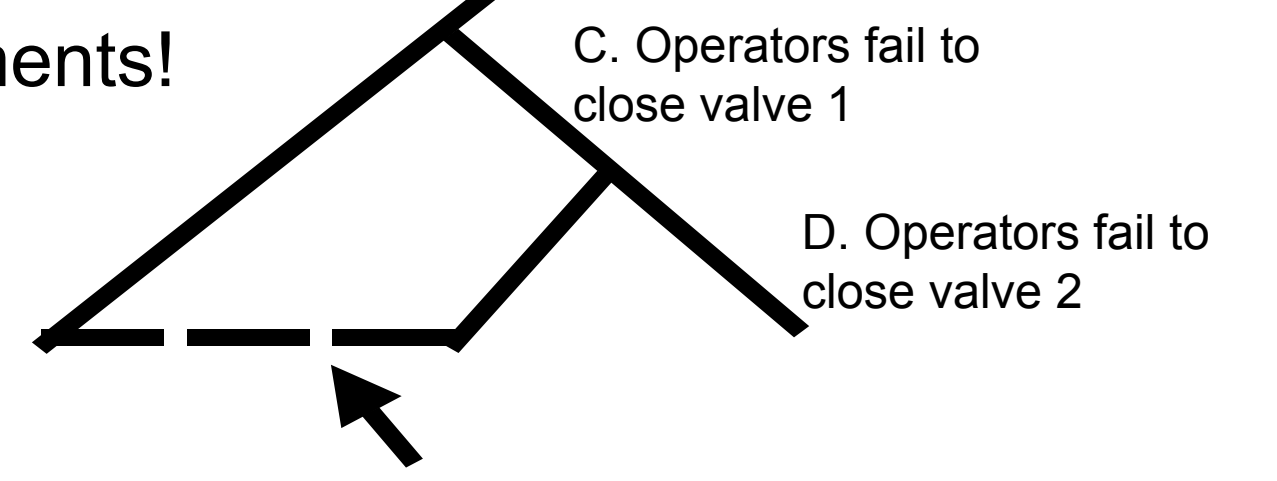

Recovery is shown as dashed line after some failure back to a success path 


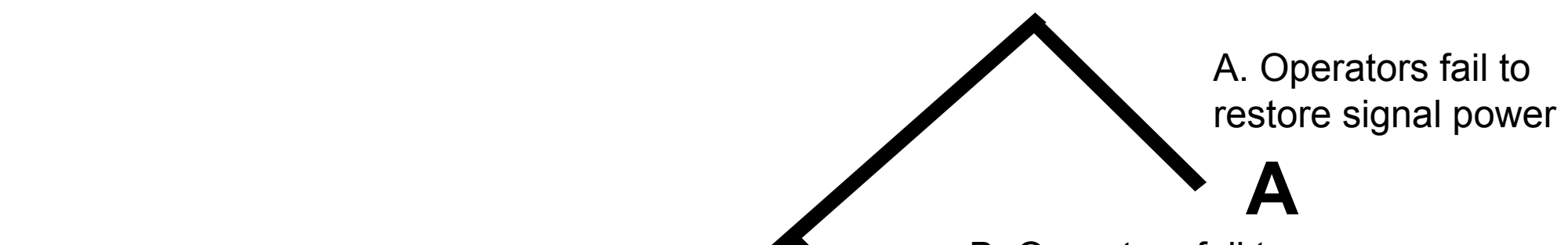

B. Operators fail to restore control power

aB

C. Operators fail to close valve 1

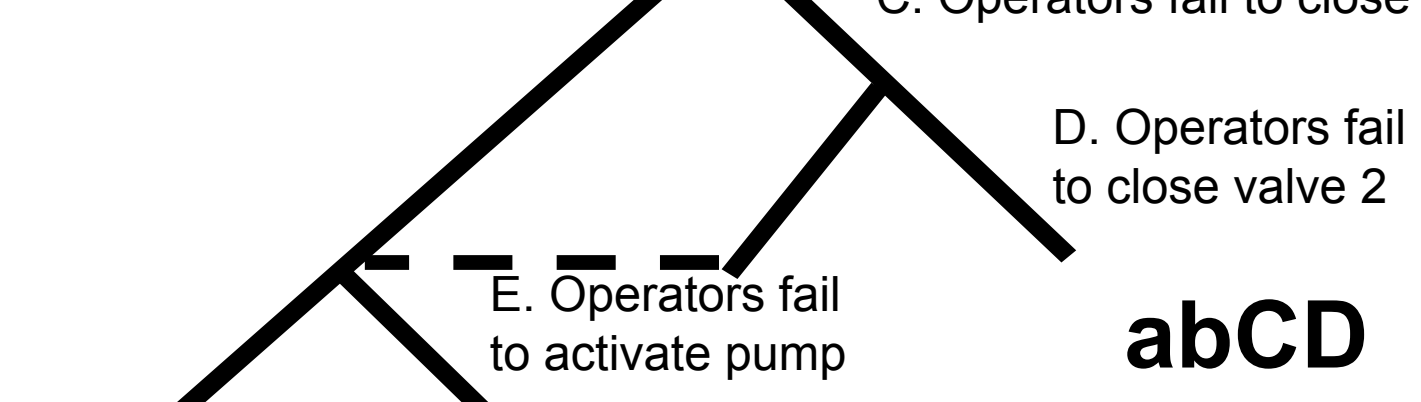

A failure path is a path starting at the top of the tree that ends in failure (i.e., $A, a B, a b C D$, abcEF, and abCdEF are all

abcEF abCdEF failure paths for this tree.) 
Success paths start at the top and end in success

\title{
abce abCde
}

\author{
abcEf \\ abCdEf
}

B. Operators fail to restore control power

A. Operators fail to restore signal power

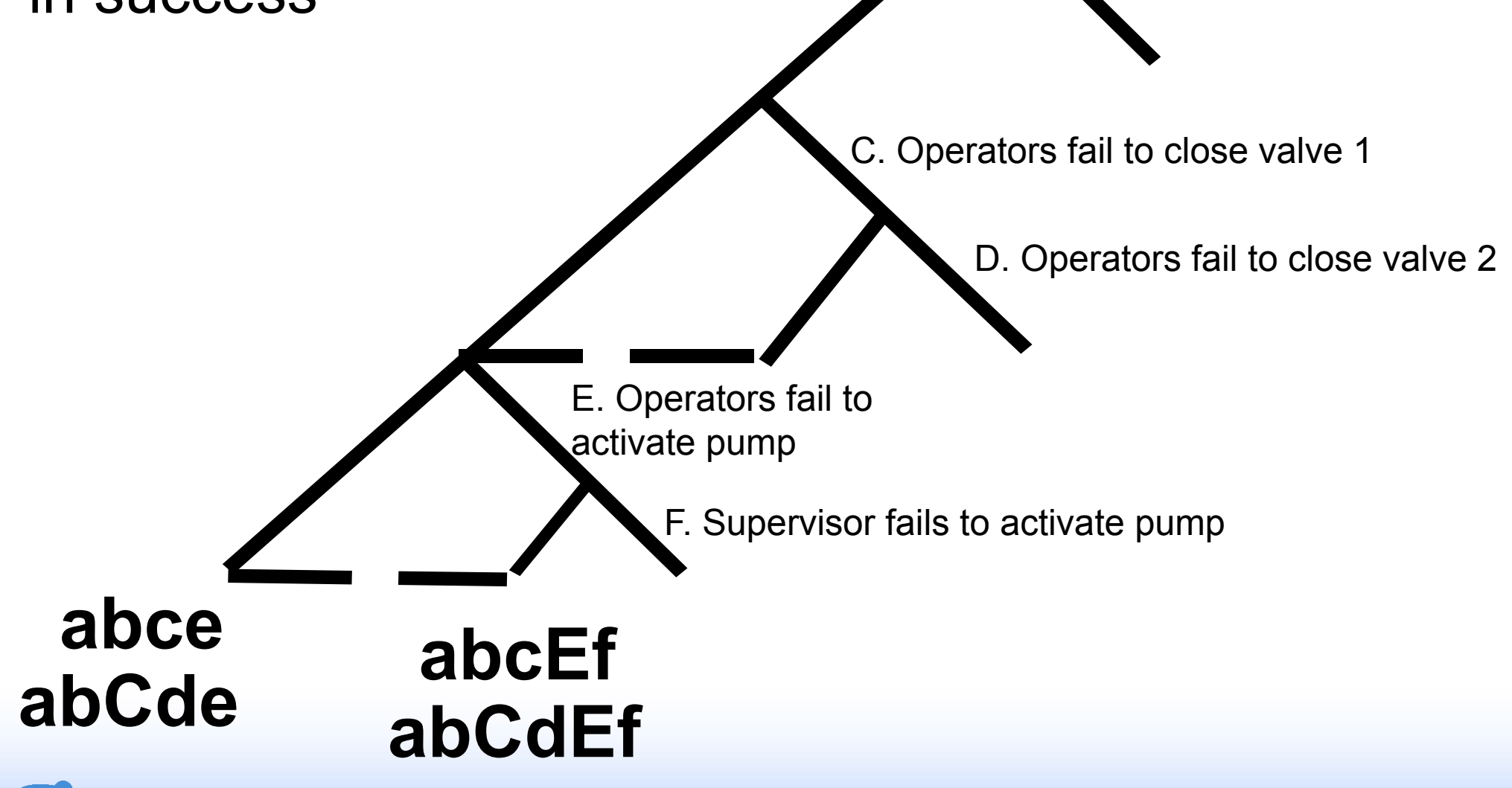




\section{Sample HRA Fault Tree}

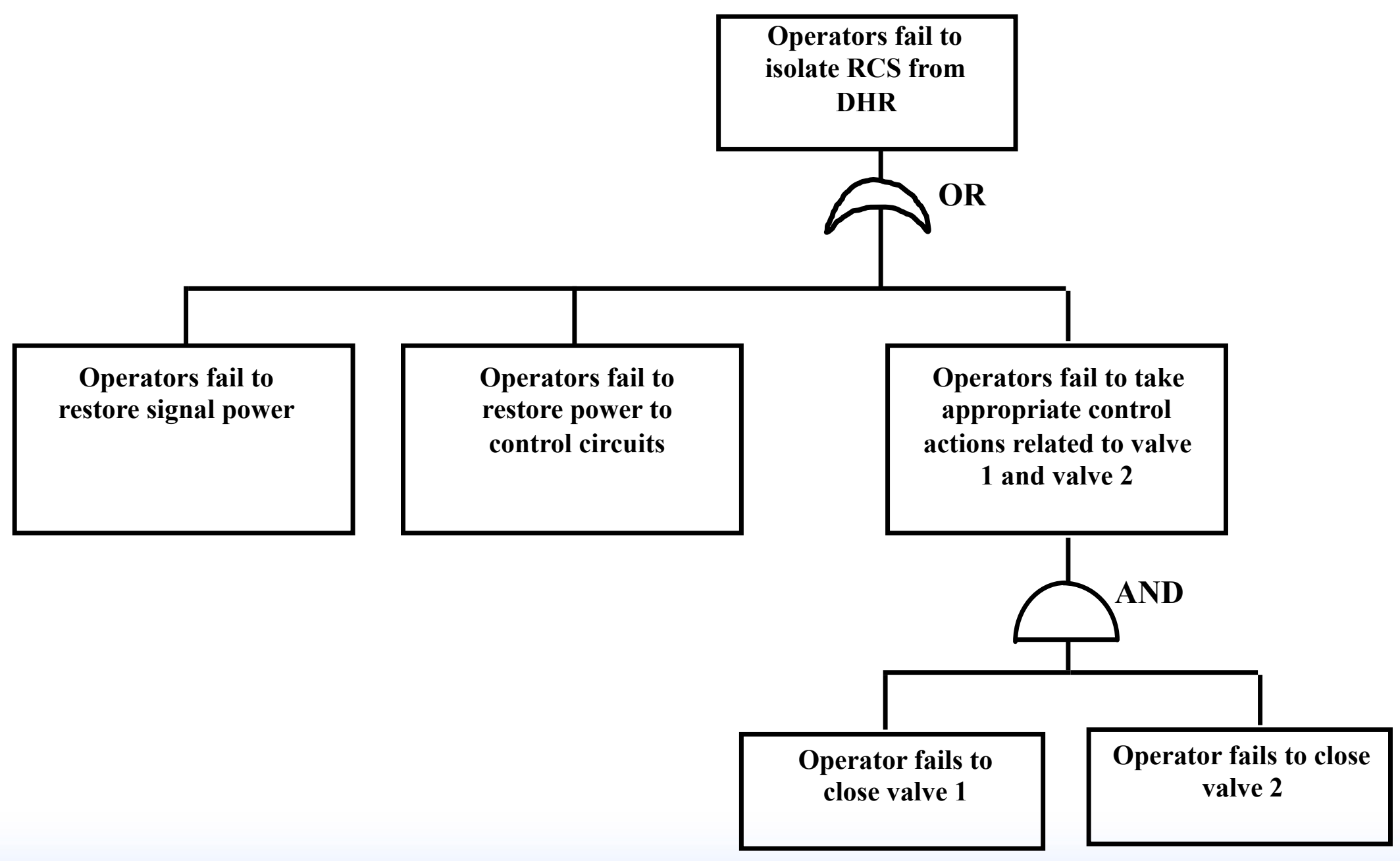




\section{HRA Fault Trees}

- Can be used to represent the same human actions and logical structures as HRA event trees

- Particularly useful in emphasizing the structure of $\underline{A N D}$ and $\underline{O R}$ logic

- Unlike HRA event trees, HRA fault trees do not do a good job of showing sequence 


\section{Exercise: HRA Fault Tree and HRA Event Tree}

- Team exercise. Get together in your teams.

- Review Appendix B on medical misadministration

- Identify one or two human failure events for the misadministration, and draw a fault tree or an event tree

- Since you didn't do a task analysis, think about which tasks are important and why, as you design your trees

- Report out and discuss 


\section{Integrating HRA into PRA Modeling}




\section{Major Approaches for Integrating HRAs into PRAs}

- SHARP/SHARP1

- IEEE 1082/D7 (1997) 


\section{Approaches Emphasize That:}

- HRA is a part of entire PRA process

- HRA personnel should be included in team

- Screening precedes selected detailed analyses

- Phases include identification, modeling, and appropriate quantification as well as documentation

- Different methods may accomplish the same thing 


\section{Systematic Human Action Reliability Procedure (SHARP1)}

- Originally developed by EPRI in mid 1980s

- Foundation for other methods

- Involves 7 basic steps and 2 decision points

- System analysts responsible for 2 steps

- HRA analysts responsible for 2 steps

- Shared responsibility for 3 steps 


\section{The SHARP Process}

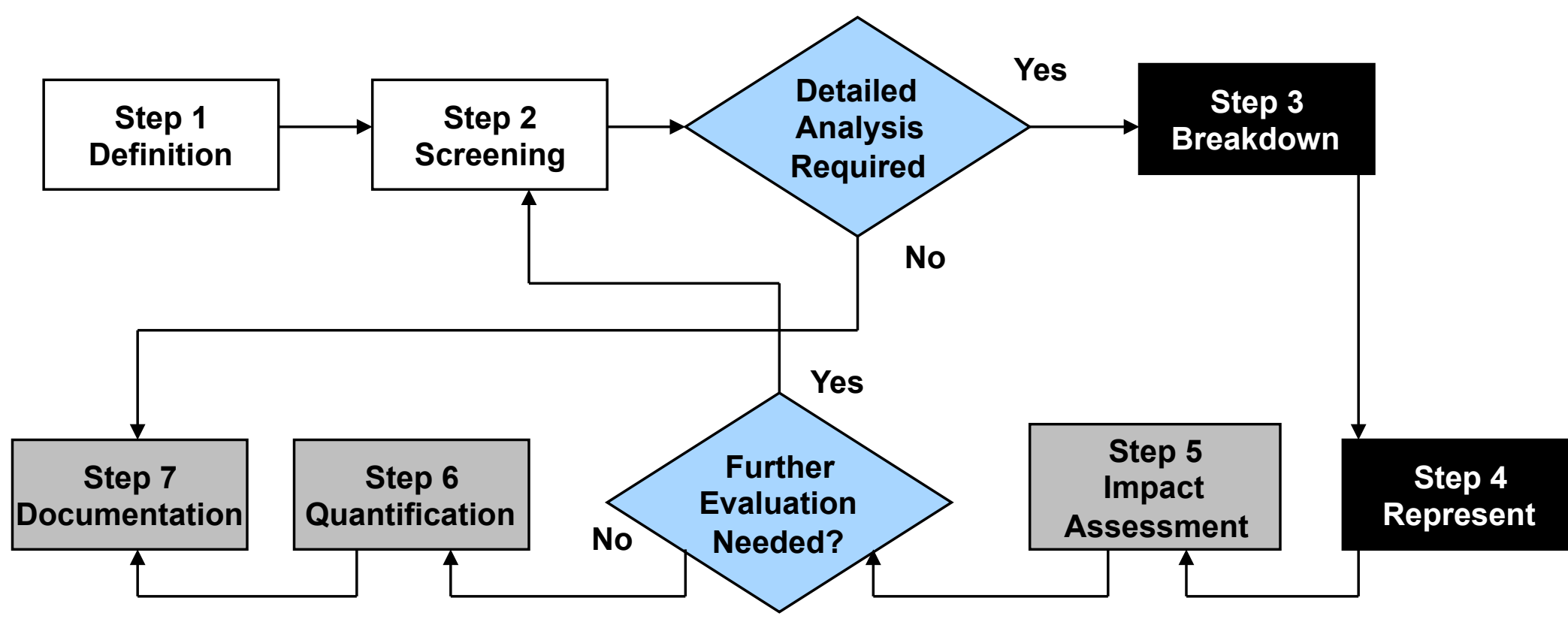

Steps 1 and 2 = Systems Analyst

Steps 3 and 4 = Human Reliability Analyst

Steps 5, 6, and $7=$ Both 


\section{IEEE STD 1082 (1997) - Guide for Incorporating Human Action Reliability Analysis for Nuclear Power Generating Stations}

- Concise document (see course CD for a copy)

- Provides general framework for integrating HRAs into PRAs

- Describes outputs and decisions entailed in the 8 steps

- Emphasizes the importance of team training 


\section{IEEE 1082 Steps}

1. Train the team

2. Familiarize team with plant

3. Build initial plant model

4. Screen human interactions

- Decision Point (Is event significant?), If no go to \#7

5. Characterize human interactions

6. Quantify human interactions

- Decision point (Is sequence recoverable?) If yes, go to \#5

7. Update plant model

8. Review results 


\section{\#5-Characterizing Human Interactions}

- Type, location and design of controls/displays

- Feedback type, sensory mode, delay, and frequency

- Characteristics of procedures used

- Task loading for control room personnel in worst case conditions

- Management and organization and supervision for maintenance

- Quality, content, frequency, and specificity of training

- Worker competency relevant to PRA scenarios 


\section{Summary of Integrating HRA in PRA}

- Two notable approaches (EPRI SHARP1 and IEEE 1082) for integrating HRA into PRA are currently available

- These approaches elaborate on the error identification, modeling, and quantification areas addressed in this course

- HRA has a role to play during the entire PRA process 


\section{LESSON 5}

\section{HRA Quantification Phase}
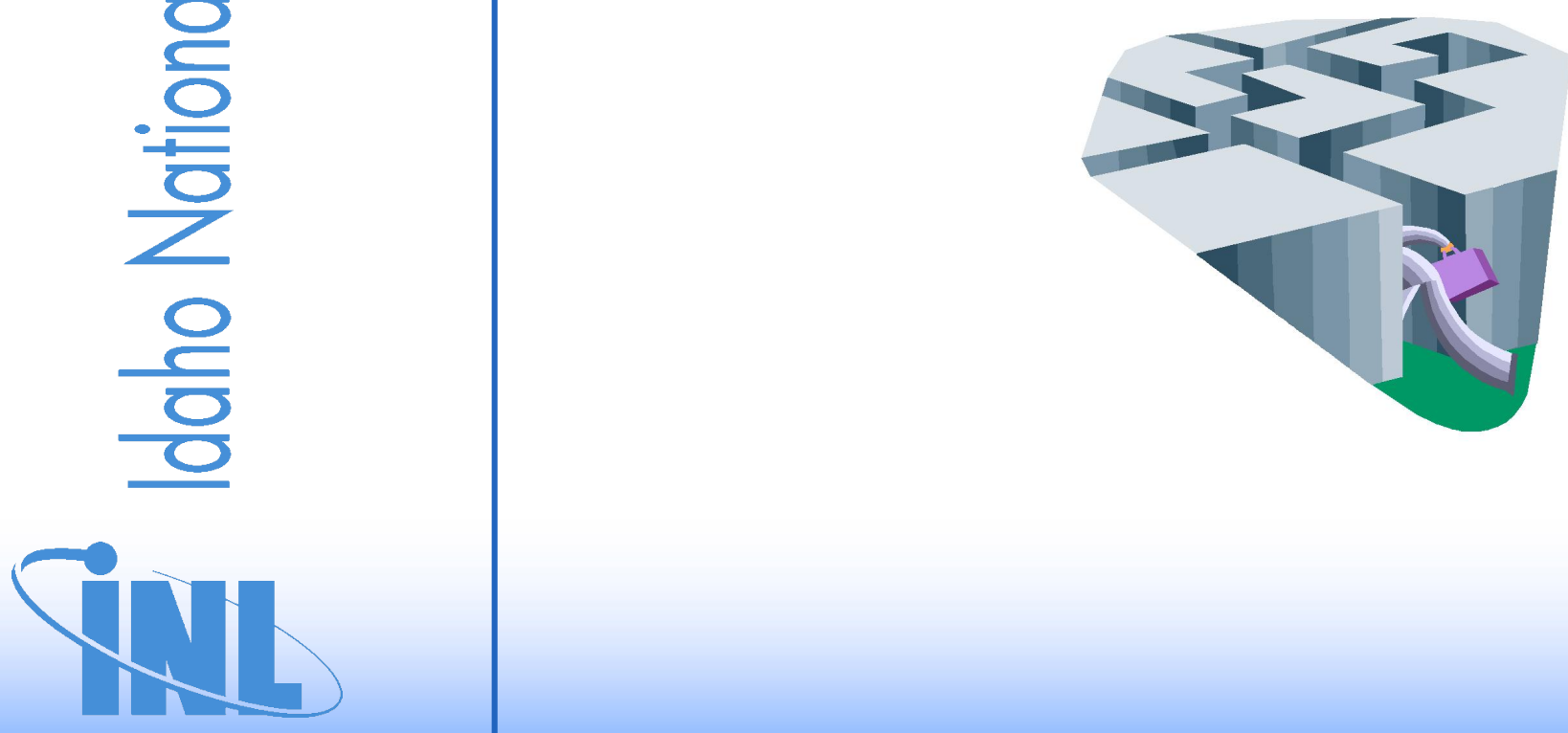


\section{Lesson 5 Objectives}

- Introduce purpose and techniques for quantifying human error

- Explain basic concepts of human error probabilities (HEPs)

- Introduce simulator and simulation techniques for quantification

- Introduce time-reliability approach

- Set stage for continuing discussion of quantification in subsequent lessons 


\section{The essential HRA processes that are integrated into PRAs - HRA Quantification}

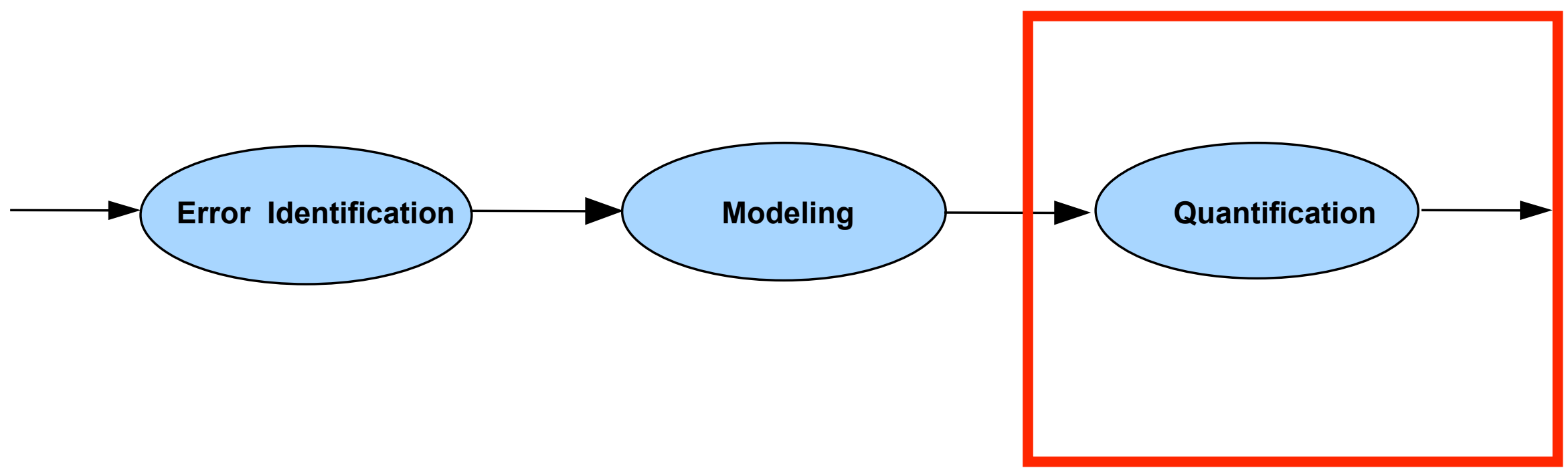




\section{Quantifying a Model}

- Quantification is a major goal of many HRAs

- Support risk-informed decision making

- Quantifying is the process of incorporating the right probabilities into a model

- The steps involved in the calculation depend on the method being used

- The data for the calculations may come from databases, simulations, expert judgment, and the HRA methods themselves

- The result is typically called a Human Error Probability (HEP)

- Various intermediate products may be created 


\section{Why Quantify HRA Models?}

- Quantification is an essential part of PRA

- Quantification promotes prioritization of prevention/ remediation activities (economic or safety analysis)

- Evaluate alternative designs

- Consider importance (risk contribution)

- Lets you address magnitude of effects 


\section{Two Levels of Precision}

1. Conservative (screening) level useful for determining which human errors are the most significant detractors from overall system safety

- An HEP for a modeled HFE may be set to a high value (e.g., 0.5) to determine if it is risk significant to the safety of the plant

2. Those found to be potentially significant contributors are analyzed in greater detail using more precise quantification 


\section{Sample HRA Quantification}

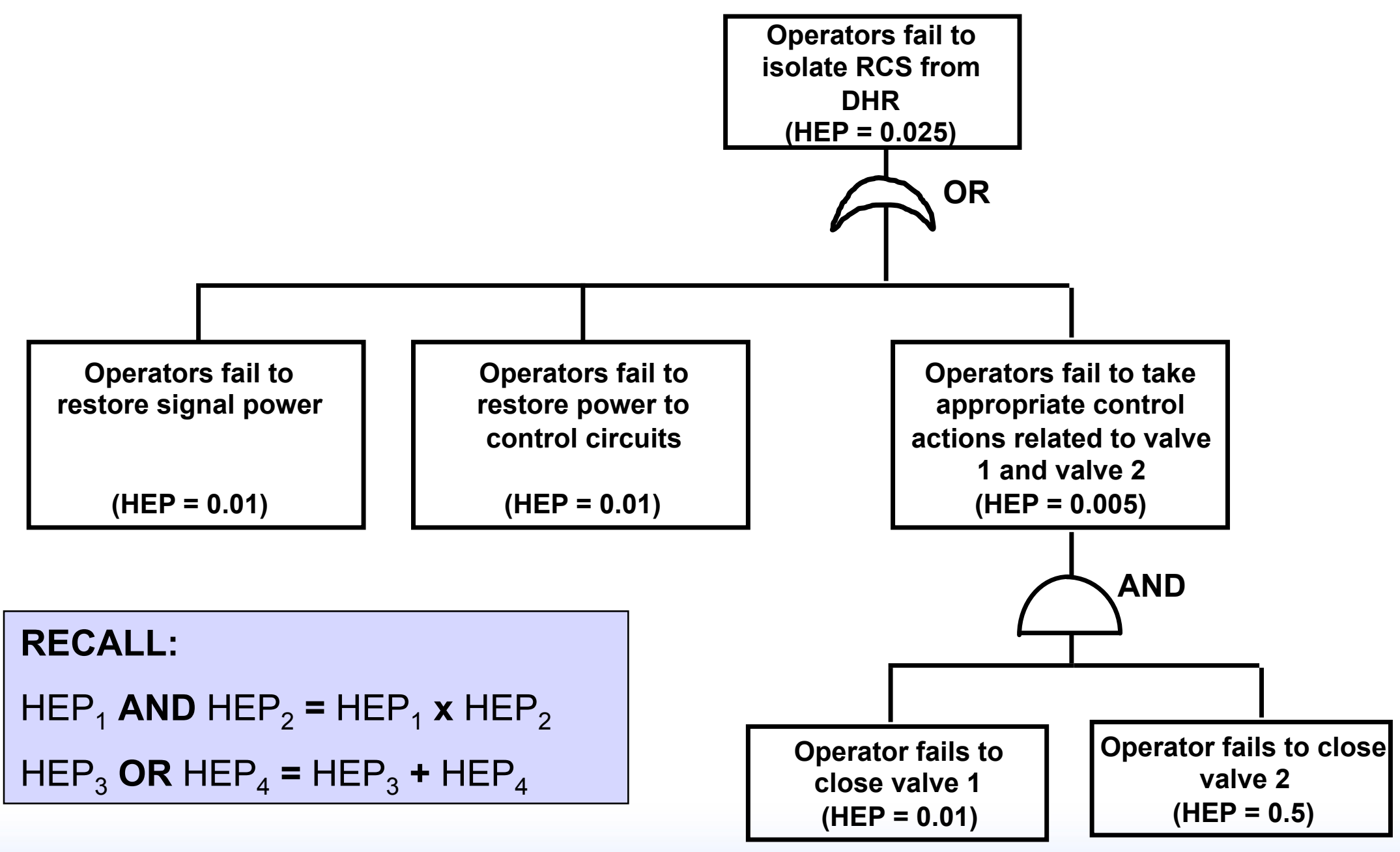




\section{Quantification Concepts}

- Base error rate

- Recovery, PSFs, and dependency modify base error rates

- Error factor (ratio of 95 th/50th or 50th/5th)

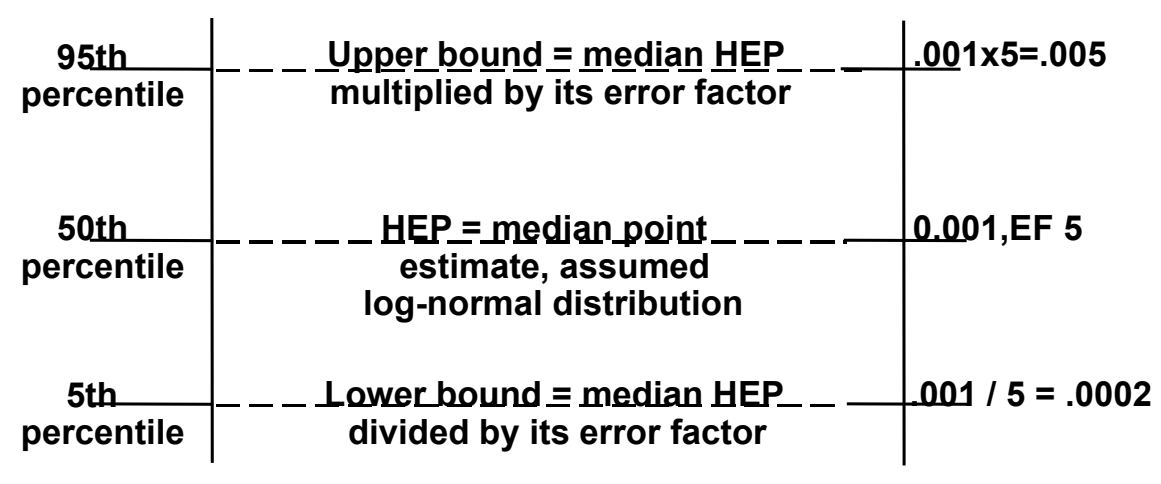




\section{Quantification Concepts (cont.)}

\section{HEP Range}

- Average or nominal performance in the range of $1 \mathrm{E}-2$ to 1E-3 (error $1 / 100$ to $1 / 1000$ times)

- Exceptionally good performance may be seen in the range of $1 \mathrm{E}-4$ to $1 \mathrm{E}-5$ (error $1 / 10,000$ to $1 / 100,000$ times)

- Poor performance may be seen in the range of 1.0 or $1 \mathrm{E}-1$ (error all the time or $1 / 10$ times)

- These values feature much lower reliability than is typical for hardware

- Temptation in regulatory framework to want to drive HEP lower, but this is not realistic 


\section{Quantification Concepts (cont.)}

Types of Quantification

- Holistic vs. atomistic approaches

- Holistic looks at the whole task to arrive at an overall HEP

- Common in expert elicitation approaches

- E.g, HEART and THERP use a type of scenario matching that looks at overall similarity between analyzed task and predefined tasks

- Atomistic looks at the drivers of the task to arrive at a computed HEP

- Typically, PSFs serve as multipliers to compute the HEP

- e.g., SPAR-H

- Note that THERP and HEART are actually somewhat hybrid approaches-they start with scenario matching but then modify that HEP on the basis of PSFs 


\section{Types of Quantification Techniques}

- Simulation and Simulator

- Time Reliability Correlation

- Expert Estimation (Lesson 6)

- HRA Methods (Lesson 7) 


\section{Simulation and Simulator Techniques}

Maintenance Personnel Performance Simulation (MAPPS)

- stochastic simulation, not widely used, mixed duration and accuracy for maintenance tasks

Cognitive Event Simulation (CES)

- developed at Westinghouse, sponsored by the NRC in the 1980s

- crews interact with a plant simulator and take actions linked to a simulation.

\section{MicroSaint}

- task analysis driven simulation

- very earliest origins were with Siegel and Wolf Model (SAINT) developed for the DoD

- Model enhanced and refined by MAAD

- basis of PHRED—NRC control room crew simulator using MicroSaint ADS/IDAC

- University of Maryland virtual plant and crew members

Many simulation techniques provide output in terms of time to complete tasks as opposed to HEPs 


\section{Use of Simulation and Simulators}

- Put the virtual back in reality!

- Simulators: real humans + virtual environments

- Simulation: virtual humans + virtual environments

- Human performance testing/determination of HEPs

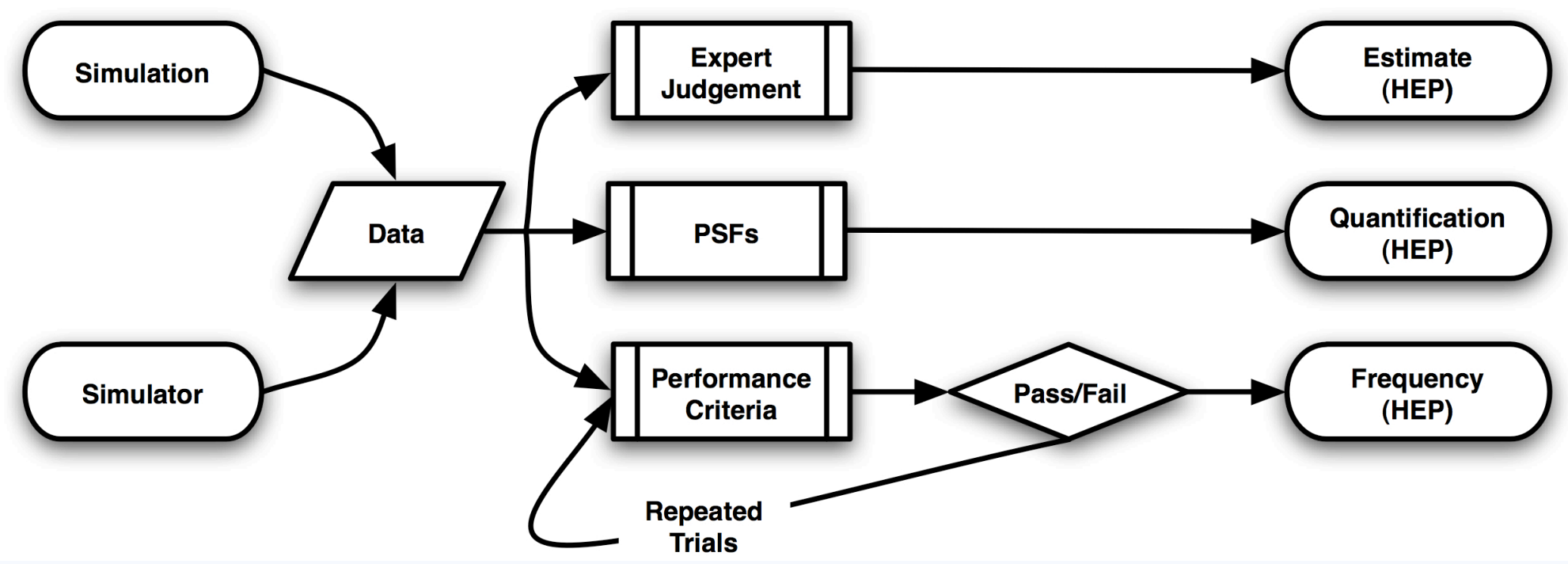




\section{Simulation for Novel Domains in HRA}

\section{Quantification through Simulation}

- Use of modeling and simulation system with virtual representation of humans to determine situations that may challenge human performance

- Process

- System extensively calibrated to human performance in known situations

- Across many Monte Carlo style trials, performance extrapolated to novel situation (e.g., longduration space flight) for which actual human performance data have not been collected

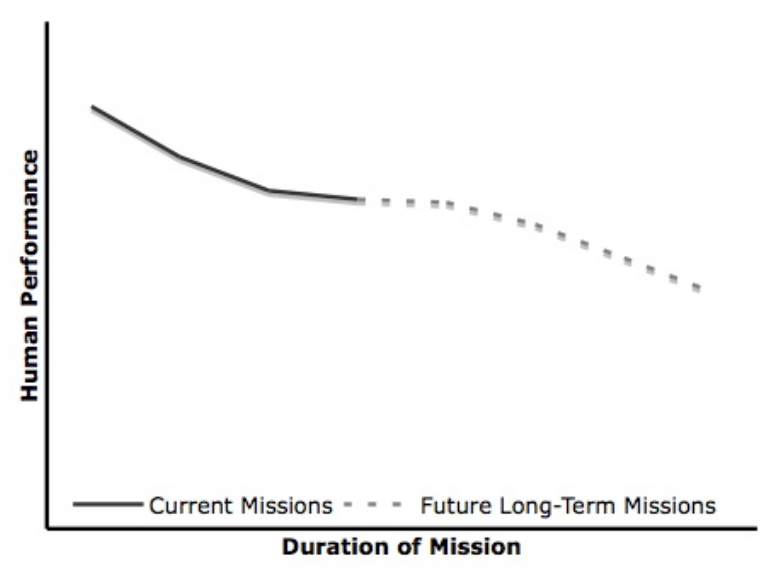

- Provides preliminary estimates of human error as well as "red flags" for situations that need to be further investigated to determine actual risk to humans or risk of human error 


\section{Time Reliability Techniques}

Human Cognitive Reliability (HCR) and Operator Reliability Experiment (ORE) are two well-known efforts

- Human error rates are estimated as a function of time

- More time means less probability of error

- Often used to estimate the probability of decision type errors

- Not a discovery method for errors of commission

- Require accurate sequence, event, and performance time estimates

- Time Reliability Curve (TRC) estimates may be adjusted for additional influences (e.g., PSFs) 


\section{Example: System/Operator Event Tree}

\begin{tabular}{|c|c|c|c|c|c|c|c|c|c|}
\hline $\begin{array}{l}\text { Initiating } \\
\text { Event }\end{array}$ & $\begin{array}{l}\text { Emerg. } \\
\text { Flow } \\
\text { Functionss }\end{array}$ & $\begin{array}{l}\text { Backup } \\
\text { Emerg. } \\
\text { Flow } \\
\text { Actuated }\end{array}$ & $\begin{array}{l}\text { Backup } \\
\text { Emerg. } \\
\text { Flow } \\
\text { Functions }\end{array}$ & $\begin{array}{l}\text { Operator } \\
\text { restores } \\
\text { PCS } \\
\text { flow }\end{array}$ & $\begin{array}{l}\text { Core } \\
\text { Emerg. } \\
\text { Makeup }\end{array}$ & $\begin{array}{l}\text { Vessel } \\
\text { Deprsrzd } \\
<\text { firewater } \\
\text { pressure }\end{array}$ & $\begin{array}{l}\text { LDW } \\
\text { purge }\end{array}$ & $\begin{array}{l}\text { Firewater } \\
\text { injection } \\
\text { (auto- } \\
\text { manual) }\end{array}$ & $\begin{array}{l}\text { Consequences } \\
\text { to Core }\end{array}$ \\
\hline RSD & EPF & BEFA & BEFF & PFRO & CEM & VDP & LDWP & FIS & \\
\hline
\end{tabular}

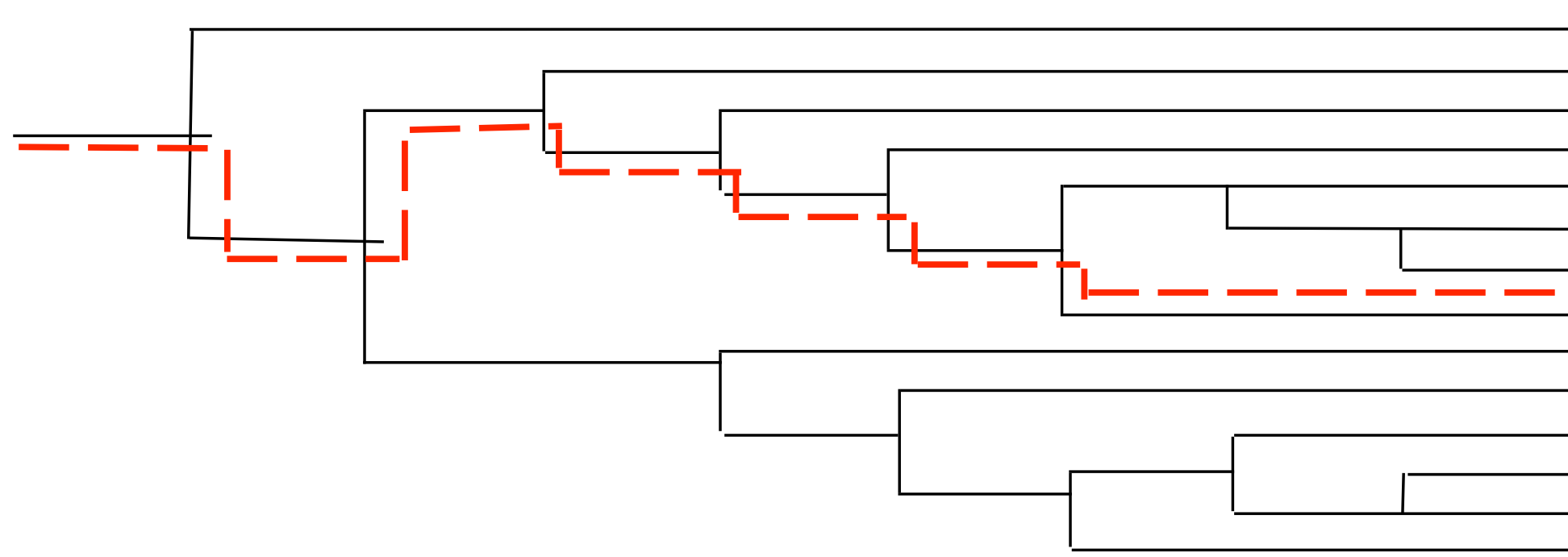

1. No Damage

2. No Damage

3. No Damage

4. No Damage

5. No Damage

6. No Damage

7. Fuel Damage

8. Fuel Damage

9. No Damage

10. No Damage

11. No Damage

12. No Damage

13. Fuel Damage

14. Fuel Damage 


\section{Calculation of Time Required and Time Available for Sequence RSD}

Time required

\begin{tabular}{|c|c|c|c|}
\hline$R S D, E P F, B E F A, B E F F$ & $3 \mathrm{~min}$. & or & $180 \mathrm{sec}$. \\
\hline PFRO & $21 \mathrm{~min}$. & or & $1260 \mathrm{sec}$. \\
\hline CEM & 20.5 min. & or & $1230 \mathrm{sec}$. \\
\hline VDP & 68 min. & or & $4080 \mathrm{sec}$. \\
\hline$\angle D W P$ & $84.5 \mathrm{~min}$. & or & 5070 sec. \\
\hline FIS & $5 \mathrm{~min}$. & or & $30 \mathrm{sec}$. \\
\hline TOTAL & $\overline{197.5 \mathrm{~min} .}$ & or & $11850 \mathrm{sec}$ \\
\hline
\end{tabular}

Initiator to core damage (6 hrs) or $21600 \mathrm{sec}$. TIME AVAILABLE Sequence events

- 11850 sec. TIME REQUIRED 9750 sec. (Time difference) $\}$ of the time 


\section{How Can You Inform an Estimate?}

- When using an HRA method or expert estimation for quantification, it is useful to anchor HEPs on actual human performance data

- NRC has developed various databases to capture human performance in nuclear power plant operations

- Nuclear Computerized Library for Assessing Reactor Reliability (NUCLARR; NUREG/CR-4639)

- Captures HEPs from previous events and other data sources

- No longer supported

- Human Factors Information System (HFIS; see CD)

- Reviews all LERs for high-level human performance contributions

- Human Event Repository and Analysis (HERA) System (NUREG/CR-6903, Volumes 1 and 2; see CD)

- Provides very detailed analysis of human performance in operating events and simulated events 


\section{HFIS and HERA}

Similarities

- Both are NRC-sponsored databases of human performance issues from reportable events at US nuclear power plants

- Both involve human reliability analysts reviewing event data and encoding according to a classification scheme

\section{Differences}

- HFIS

- High-level human performance issues for trending

- Production mode, whereby all suitable IRs and LERs are screened

- HERA

- Detailed human performance analyses for informing error/risk estimation across HRA methods

- Sampling of selective events, not production mode

- Use of potentially diverse range of sources

HFIS and HERA serve complementary roles for capturing human performance data 


\section{HERA and HRA}

\section{HERA Complements Existing HRA Approaches}

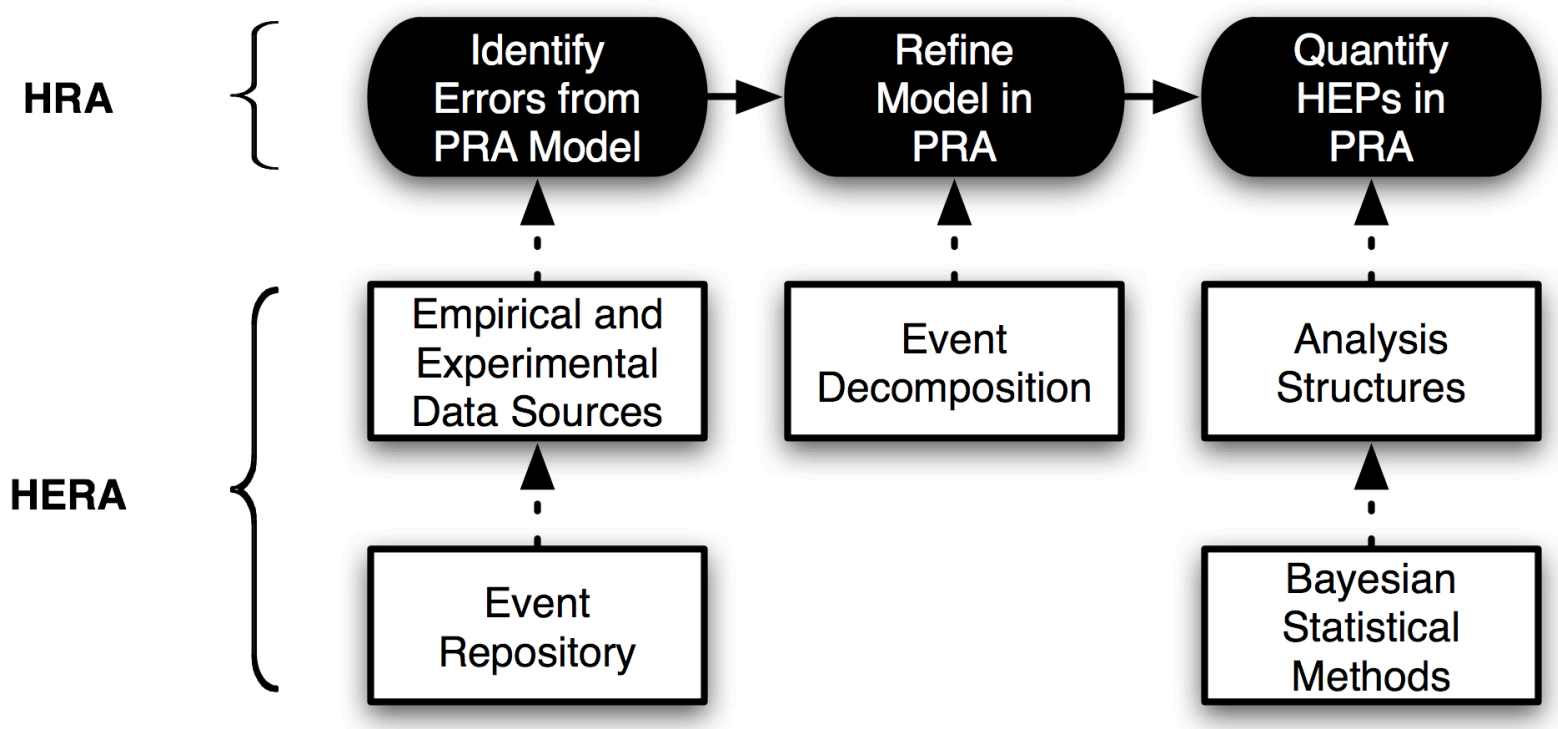

- Provides a much-needed detailed decomposition of human-related events at nuclear power plants

- Achieves cross-method flexibility

- Complements existing HFIS database 


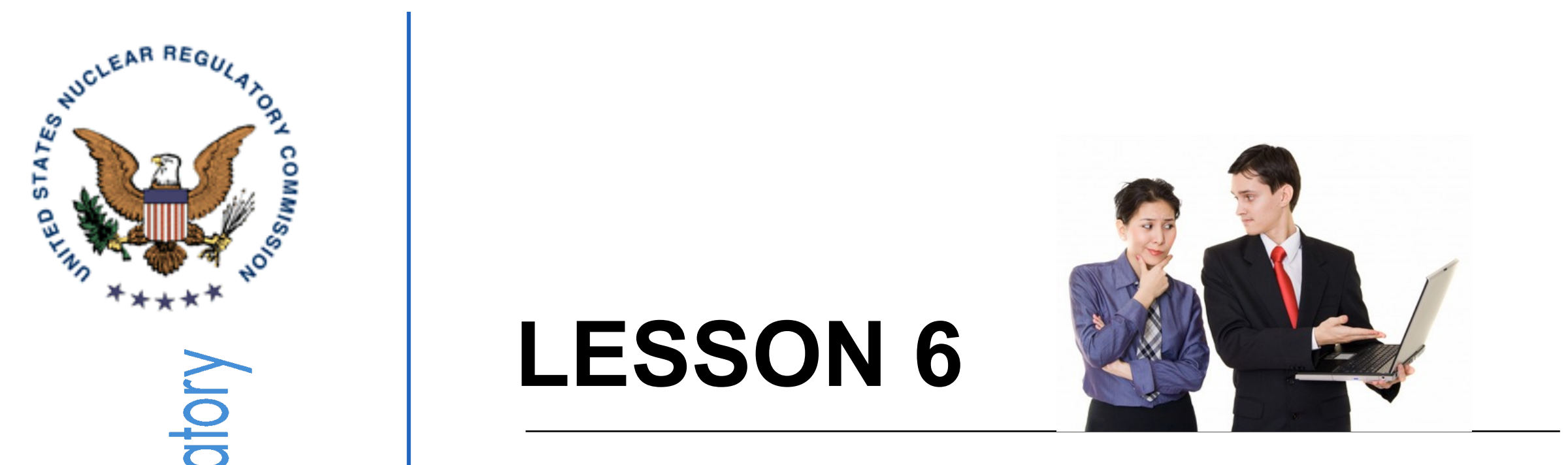

\section{Expert Estimation}




\section{Lesson 6 Objectives}

- Introduce basic approach to using expert estimation for quantification of HEPs

- Make aware of strengths and limitations of expert estimation

- Give hands-on example of how to apply expert estimation 


\section{Exercise: Expert Estimation}

- Estimate how many beans there are in the bean jar

- Report your estimate and discuss 


\section{Some Expert Estimation Techniques}

- Nominal Group Technique (NGT)

- Delphi Technique

- Success Likelihood Index Method (SLIM)

- Meyer and Booker Compendium (NUREG/CR-5424; see CD)

- ATHEANA (NUREG-1880; see CD)

- ASP Program Simplified Expert Elicitation Guideline (see CD) 


\section{Why Expert Estimation?}

- Existing HRA quantification approaches do not cover the scenario or HFE at hand

- There is insufficient empirical (experimental or operational) data from which to form a frequency estimate

- There are subject matter experts available to inform the quantification

- Some HRA methods use expert estimation as the method of choice 


\section{Issues with Expert Estimation}

- Subject matter experts may not be experts at producing probabilities

- Generally, humans are not skilled at translating mental representations into quantities

- Quality of information presented to the expert can greatly affect estimate

- Experts often do not agree

- In a group setting, one expert may dominate or influence others

- In a group setting, it may be difficult to reach consensus

- Experts may not be calibrated to the same numeric scaleeven if they actually agree, they may not generate the same result 


\section{Improving Expert Elicitation}

- Need to recognize that knowledge is gained from reason, intuition, experiences

- Harness multiple, qualified experts

- Provide sufficient background and issue familiarization to appropriate level of detail

- Reproducibility important

- Document all assumptions and processes

- Emphasize accountability

- Experts should be willing to "sign off" on estimates

- Provide training and calibration of experts to the extent possible

- Try to avoid exaggerated illusion of precision

- An expert-generated HEP should not be a substitute for empirically derived data

- Estimation should be an iterative process

- Have experts review and revise results 


\section{Calibrating Experts}

\section{Possible Calibration Points (from ATHEANA User's Guide, NUREG-1880)}

\begin{tabular}{|l|c|l|}
\hline \multicolumn{1}{|c|}{ Circumstance } & Probability & \multicolumn{1}{|c|}{ Meaning } \\
\hline The operator(s) is "Certain" to fail & 1.0 & $\begin{array}{l}\text { Failure is ensured. All } \\
\text { crews/operators would not } \\
\text { perform the desired action } \\
\text { correctly and on time. }\end{array}$ \\
\hline The operator(s) is "Likely" to fail & $\sim 0.5$ & $\begin{array}{l}\text { Sout of } 10 \text { would fail. The } \\
\text { level of difficulty is } \\
\text { sufficiently high that we } \\
\text { should see many failures if } \\
\text { all the crews/operators were } \\
\text { to experience this scenario. }\end{array}$ \\
\hline The operator(s) would "Infrequently" fail & $\sim 0.1$ & $\begin{array}{l}1 \text { out of } 10 \text { would fail. The } \\
\text { level of difficulty is } \\
\text { moderately high, such that } \\
\text { we should see an occasional } \\
\text { failure if all of the } \\
\text { crews/operators were to } \\
\text { experience this scenario. }\end{array}$ \\
\hline The operator(s) is "Unlikely" to fail & $\begin{array}{l}1 \text { out of } 100 \text { would fail. The } \\
\text { level of difficulty is quite low } \\
\text { and we should not see any } \\
\text { failures if all the } \\
\text { crews/operators were to } \\
\text { experience this scenario. }\end{array}$ \\
\hline The operator(s) is "Extremely Unlikely" to fail & $\sim 0.001$ & $\begin{array}{l}1 \text { out of } 1000 \text { would fail. } \\
\text { This desired action is so easy } \\
\text { that it is almost } \\
\text { inconceivable that any } \\
\text { crew/operator would fail to } \\
\text { perform the desired action } \\
\text { correctly and on time. }\end{array}$ \\
\hline
\end{tabular}




\section{Exercise: Expert Estimation}

Use the ATHEANA anchor values to estimate these likelihoods:

- You take a wrong turn while driving to work

- You run a red light while turning left at an intersection

- You get off at the wrong metro stop on the way to class

- You miss an important text message from a friend because you are so engrossed in the instructor's lecture

- You forget to send an attachment with an email to your manager

What factors weighed into your decision? 


\section{Example: NRC Expert Estimation Guideline}

Expert elicitation is needed for cases when:

- There are infrequent events that are not included in PRA or HRA models

- There is inadequate operational or experimental data to arrive at probabilistic estimates

Expert elicitation methods may be:

- Costly

- Time-consuming

- Not always tractable

Need an expert elicitation approach that is:

- Cost effective

- Quick to meet Significance Determination Process (SDP) and Accident Sequence Precursor (ASP) deadlines

- Scrutable 


\section{Guideline Overview}

Worksheet Based Approach for Hardware (PRA) or Human (HRA)

\section{Events (see CD)}

- Background Information/Problem Framing

- Individual Expert Elicitation

- Aggregation

- Consensus

- Panel

- Mathematical

- Checklist

\begin{tabular}{c|c|c|}
\multicolumn{1}{c}{} & \multicolumn{1}{c}{$\begin{array}{c}\text { Hardware } \\
\text { Failure }\end{array}$} & \multicolumn{1}{c}{$\begin{array}{c}\text { Human } \\
\text { Error }\end{array}$} \\
\cline { 2 - 3 } $\begin{array}{c}\text { Problem } \\
\text { Framing }\end{array}$ & Appendix A & Appendix A \\
\cline { 2 - 3 } $\begin{array}{c}\text { Expert } \\
\text { Elicitation }\end{array}$ & Appendix B & $\begin{array}{c}\text { SPAR-H } \\
\text { Worksheets }\end{array}$ \\
\cline { 2 - 3 } $\begin{array}{c}\text { Estimate } \\
\text { Aggregation }\end{array}$ & Appendix C & Appendix D \\
\cline { 2 - 3 } & Elicitation \\
\cline { 2 - 3 } Checklist & Appendix E & Appendix E \\
\cline { 2 - 3 } & &
\end{tabular}




\section{Expert Estimation for Hardware}

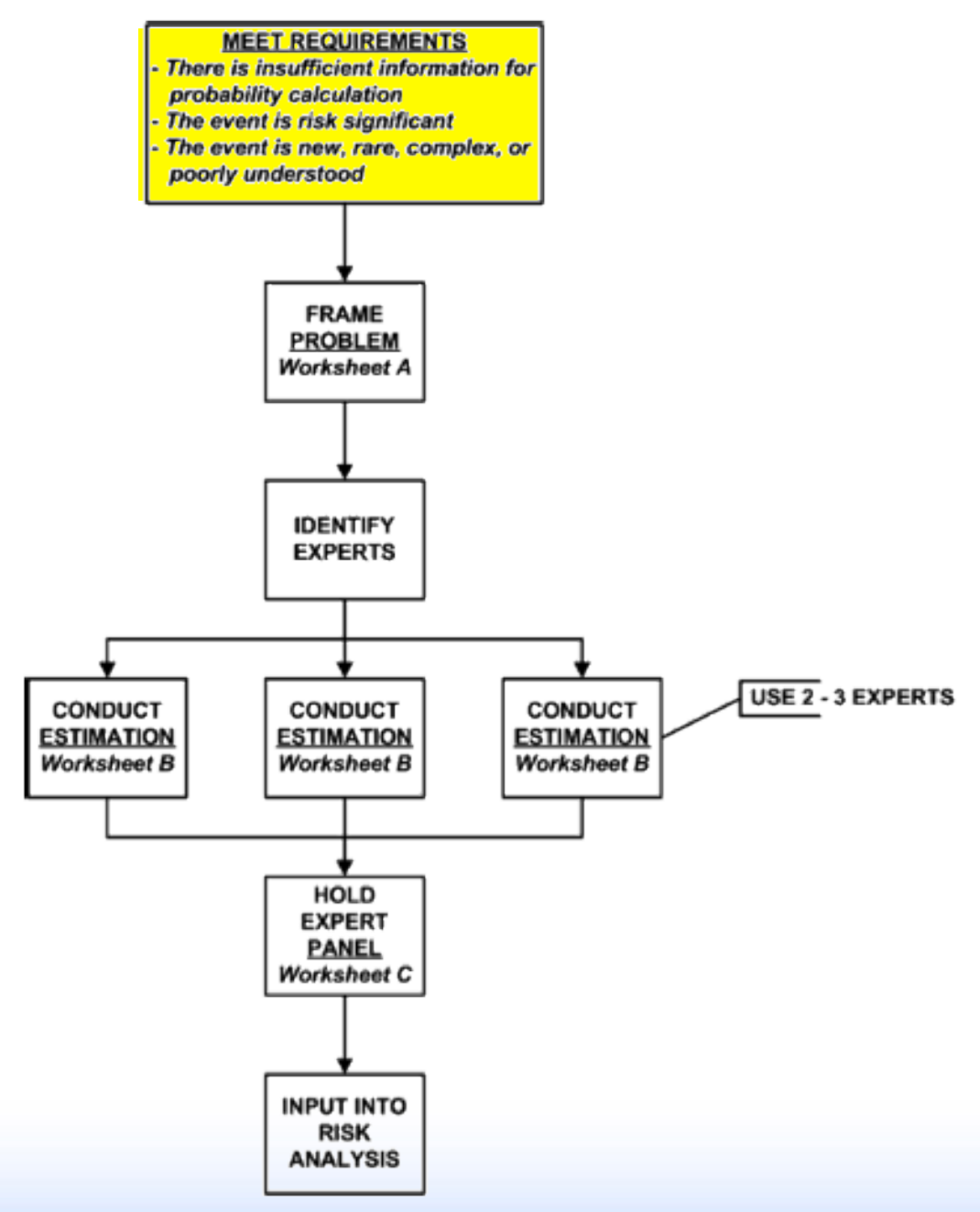




\section{Expert Estimation for Hardware}

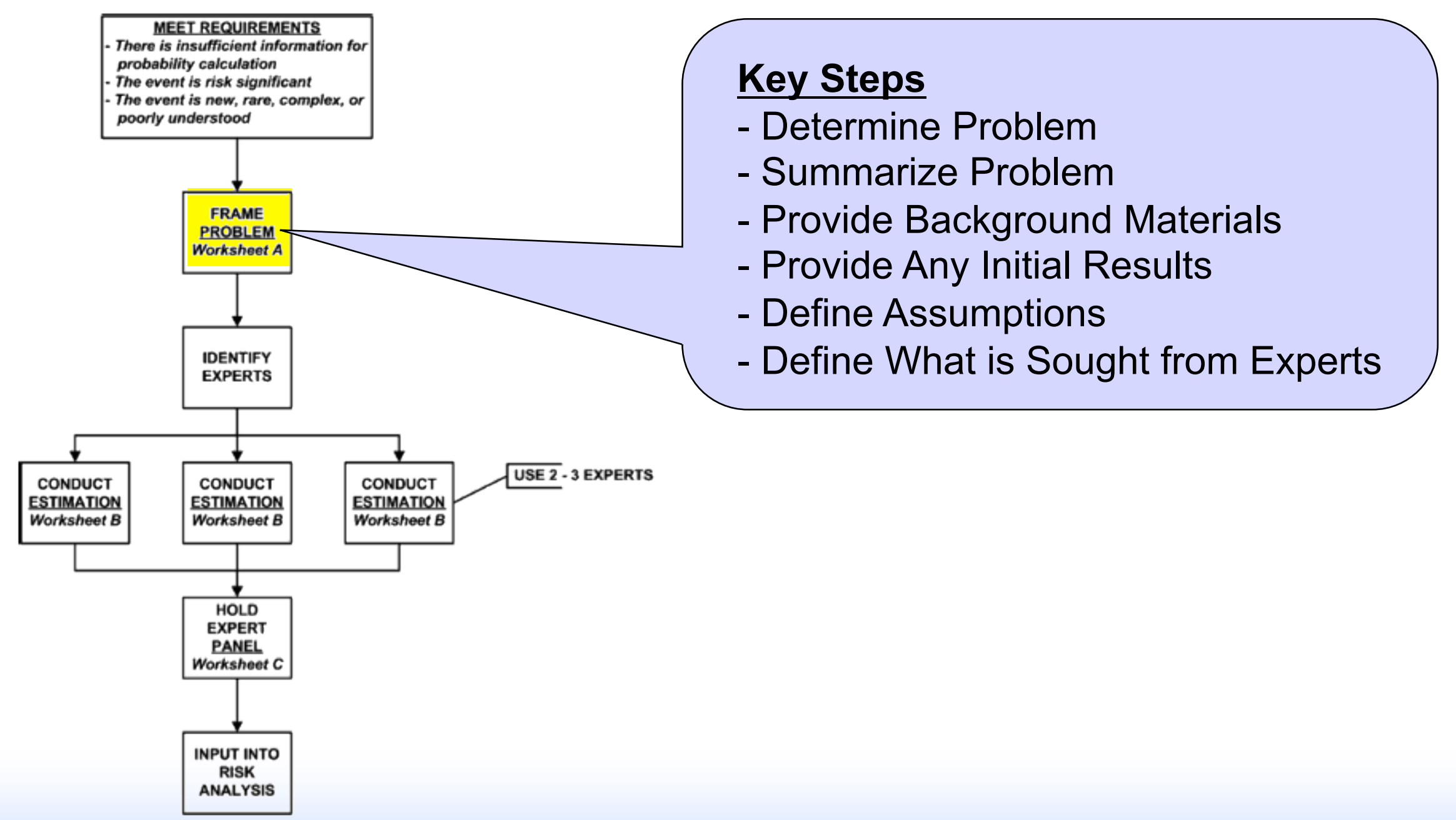




\section{Expert Estimation for Hardware}

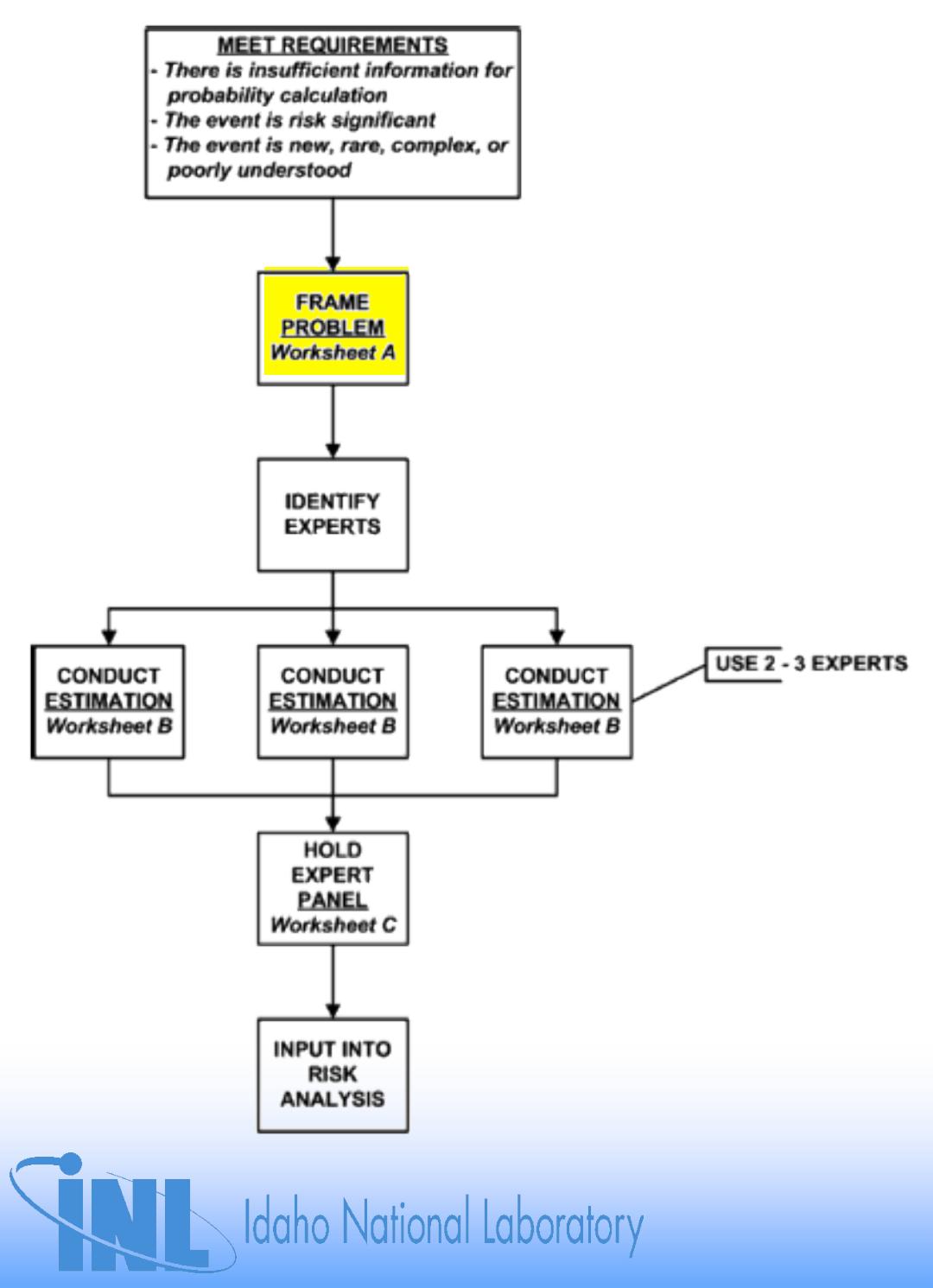

APPENDIX A

EXPERT ELICITATION BACKGROUND INFORMATION WORKSHEET

Instructions. Complete this worksheet priar to contacting the expert. Provide this worksheet and supporting materials to present the problem domain to the expert. All experts should receive identical information.

1. Analyst's Name and NRC Affiliation:

2. Problem Type: $\square$ Actual Hardware Failure $\square$ Latent Hardware Failure $\square$ Other: $\square$ Actual Human Error $\square$ Latent Human Error

-

3. Summary of Problem for Analysis:

4. Supporting Documents (Attached):

5. Summary of Results from Initial Analysis:

6. Analysis Assumptions:

7. Information Required from Expert: 


\section{Expert Estimation for Hardware}

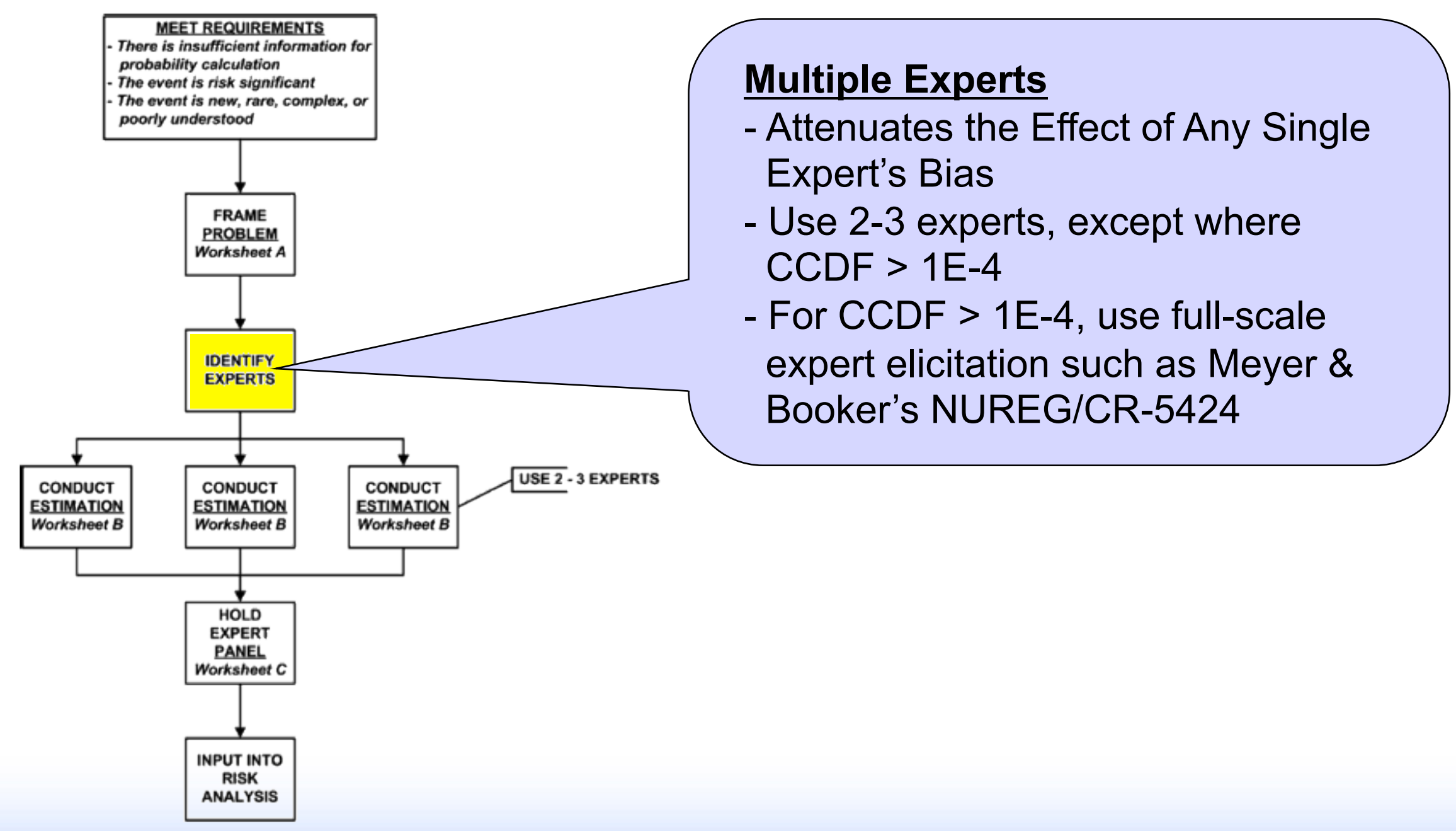




\section{Expert Estimation for Hardware}

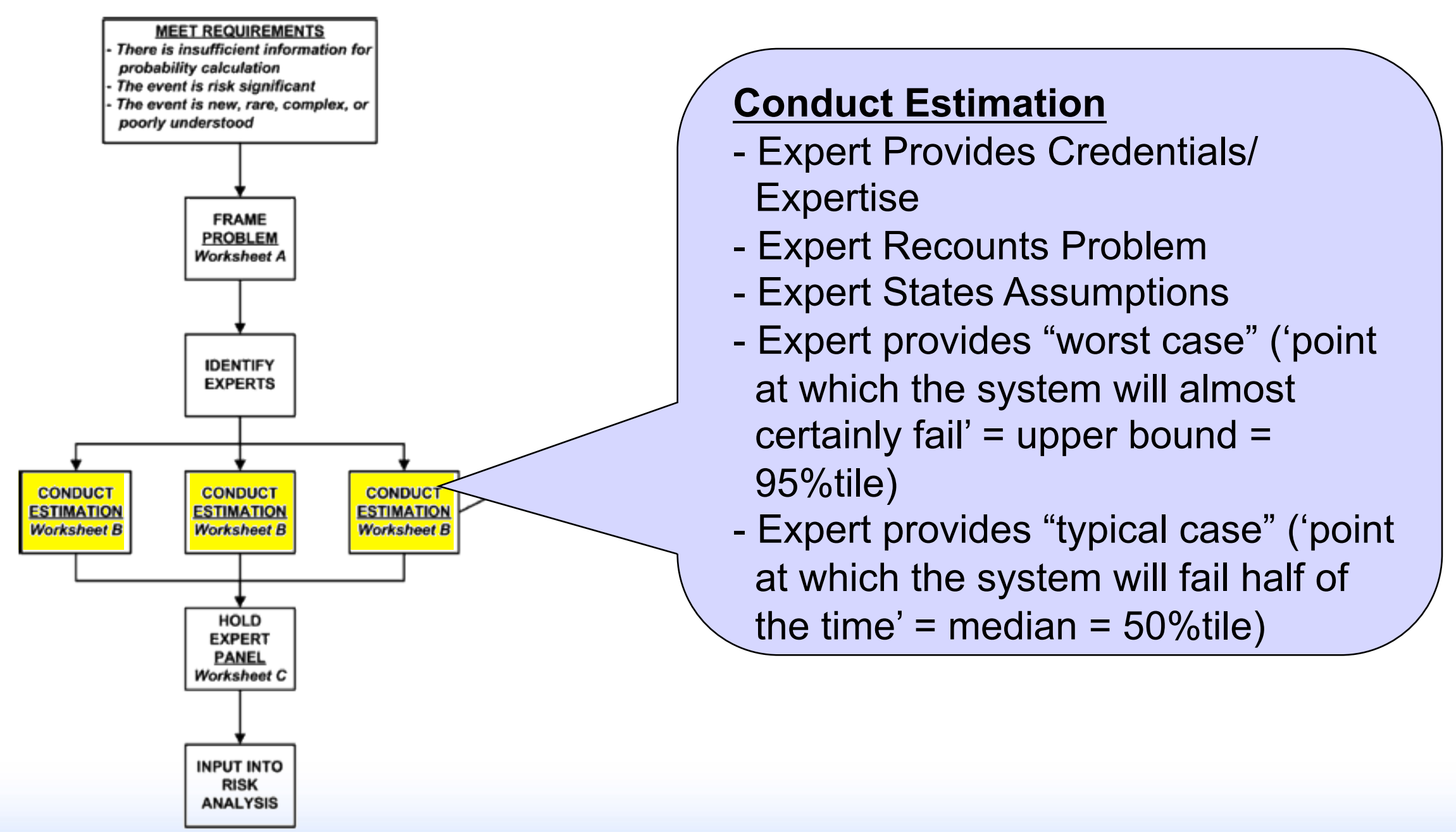




\section{Expert Estimation for Hardware}

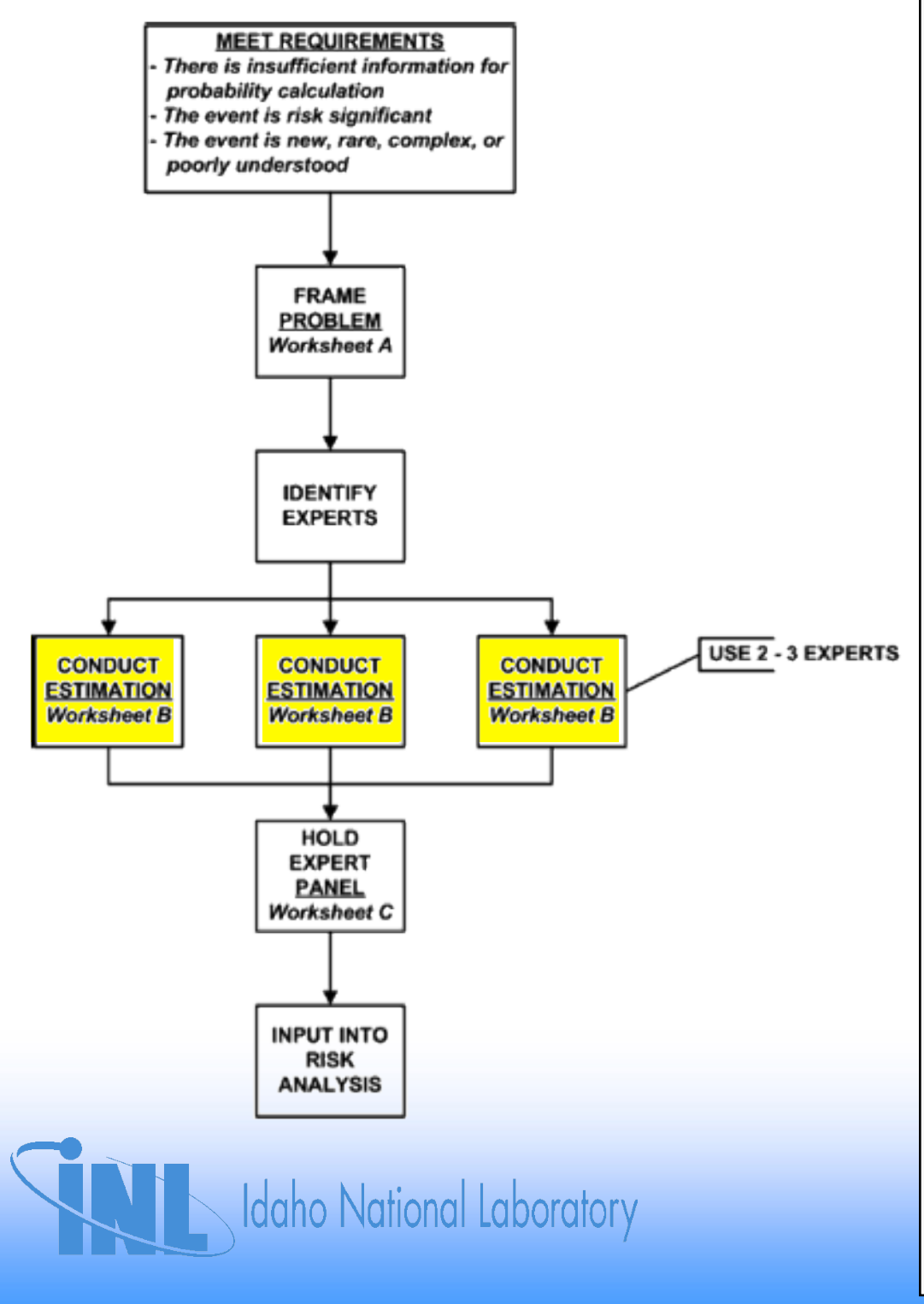

APPENDIX B

\section{EXPERT ELICITATION WORKSHEET FOR HARDWARE FAILURE}

Instructions. Complete this worksheet for each individual expert. Begin by answering any questions the expert has regarding the problem being analyzed. Then step through each question in sequence. Attach any supporting materia's provided by the expert.

1a. Date of Elicitation: __ _ _ 1 1b. Time of Elicitation: __ _ _ A.M. $/$ 口 P.M.

2. Expert's Name and Affillation:

a In-house NRC a Industry Consultant a Academia $\quad$ Other:

a National Lab/DOE a Licensee a Vendor

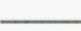

3. Expert's Areas of Expertise Relevant to Analysis:

4. Expert's Comments on Problem Under Analysis:

. Median Falure Rate/ Percent of Time There's a 50/50 Likelihood of Hardware Fallure:

6. Upper Bound/Percent of Time That Hardware WIII Almost Certainly Fail:

7. Factors Shaping Expert Estimate:

8. Additional Comments by Expert: 


\section{Expert Estimation for Hardware}

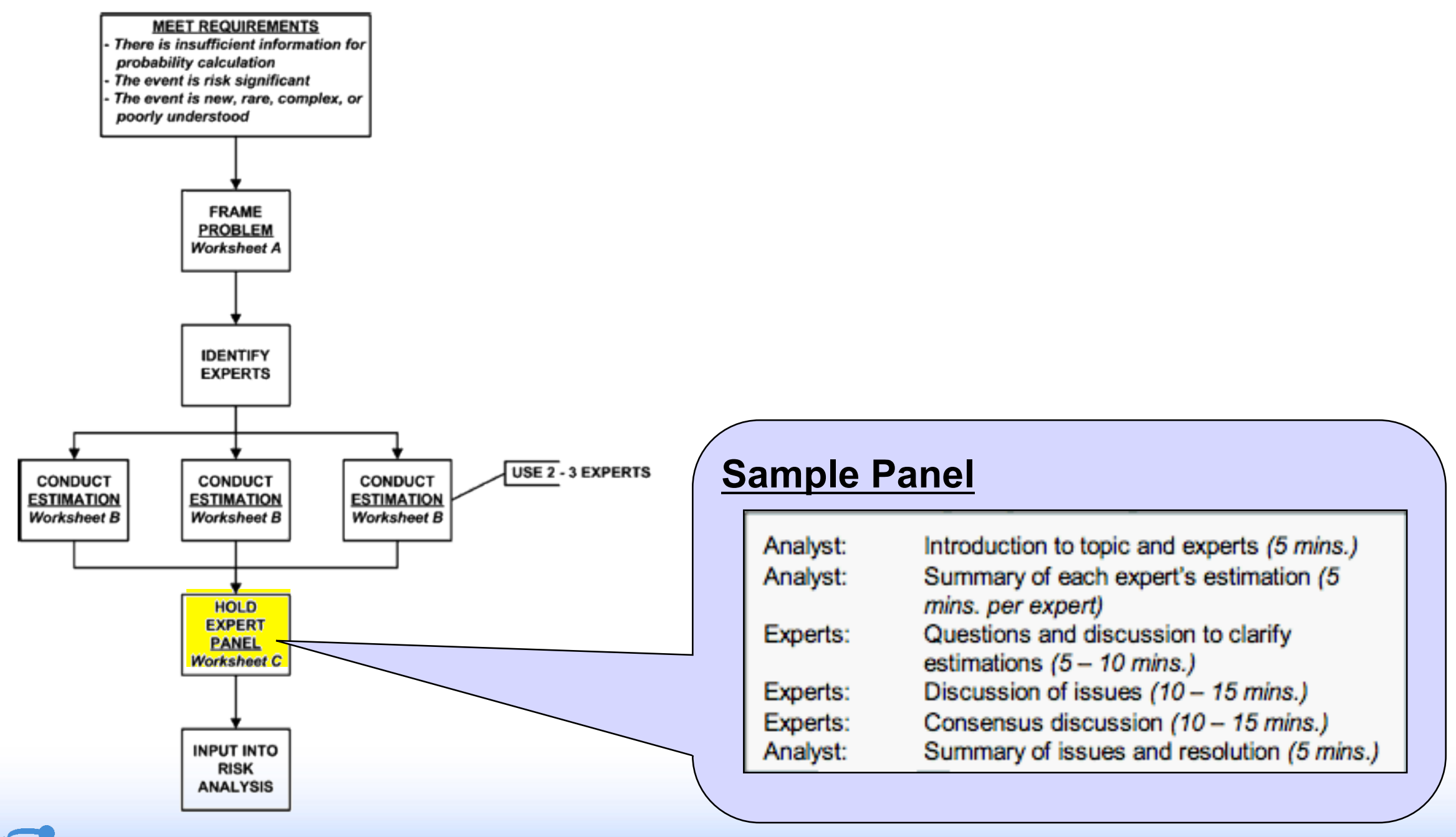




\section{Expert Estimation for Hardware}

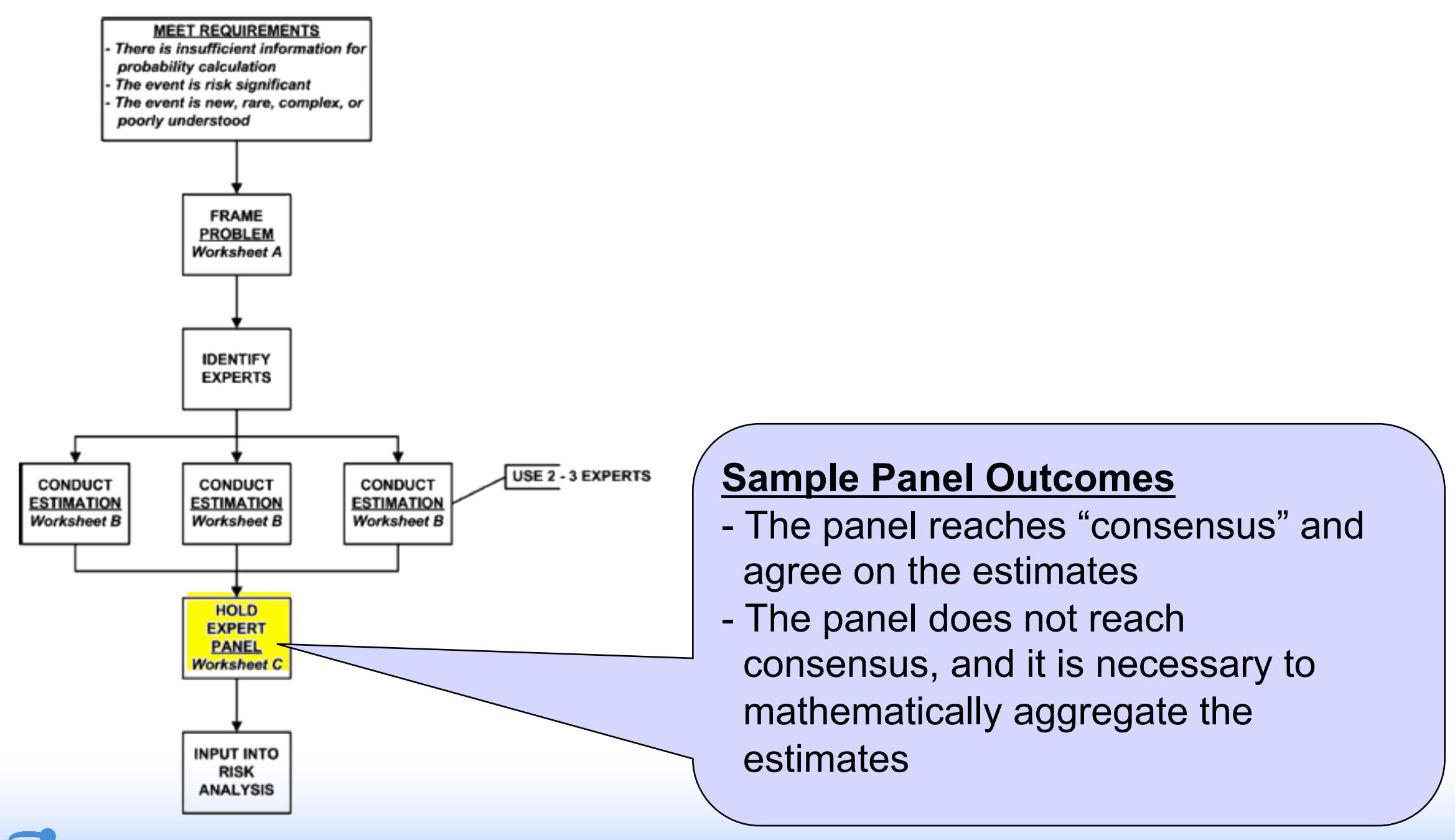




\section{Expert Estimation for Hardware}

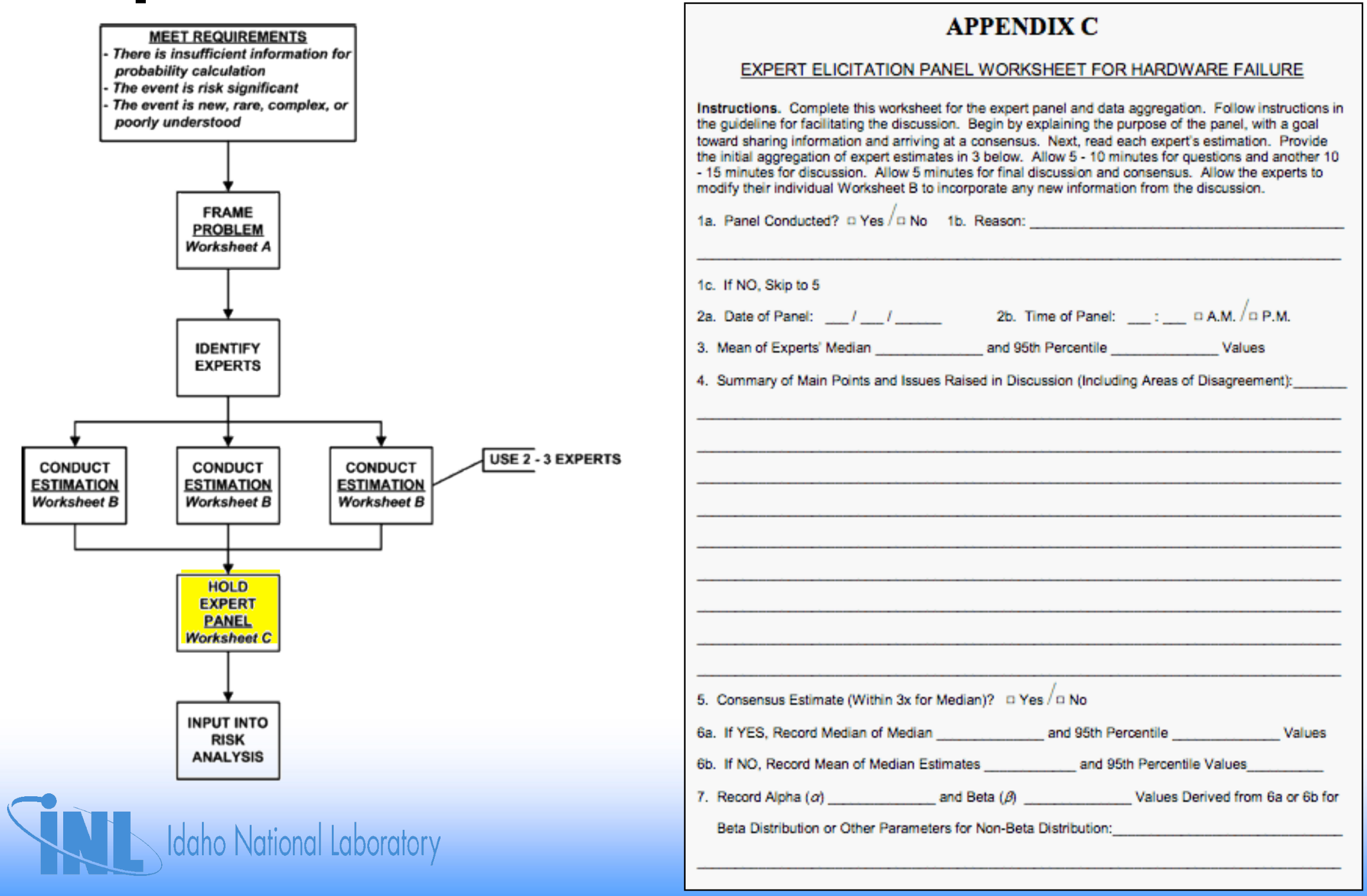




\section{Expert Estimation for Hardware}

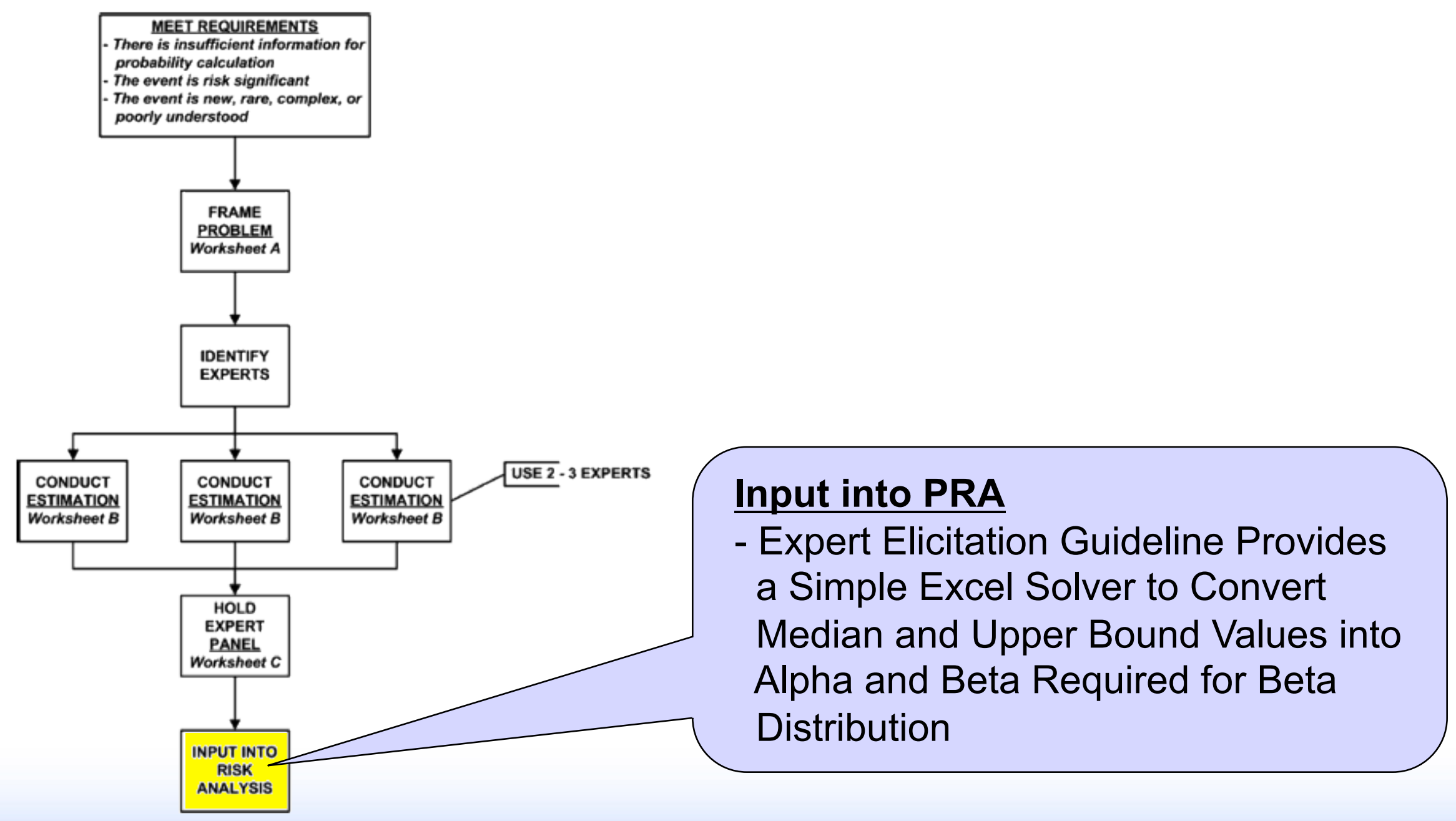




\section{Expert Estimation for Human Error}

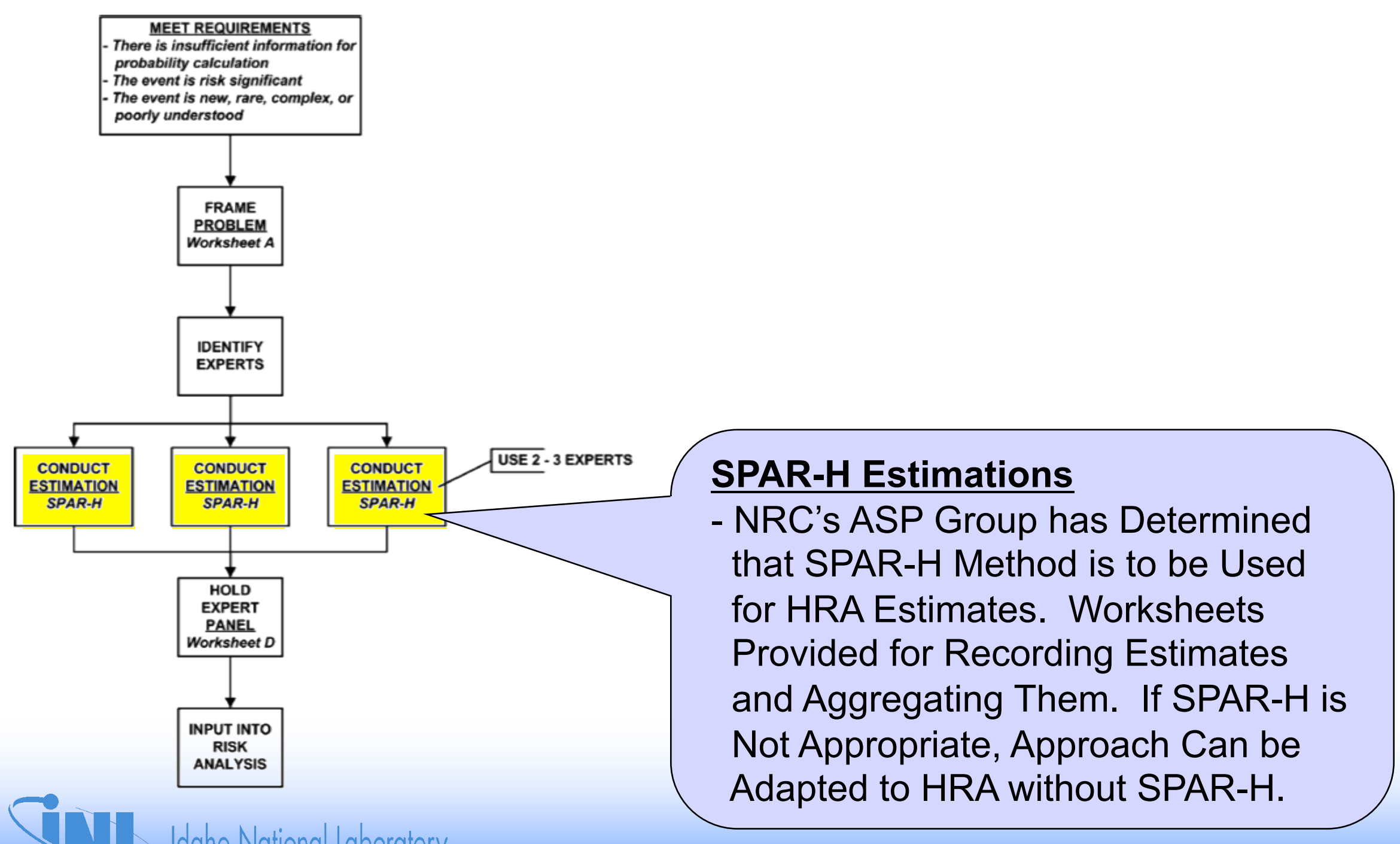




\section{Validation}

\section{Methodological Validation}

- Method derived from interviews with 20 ASP and SDP analysts

- Three iterations of guideline with NRC peer review

\section{Implementational Validation}

- PRA case study on incident involving air in HPSI pumps at Palo Verde Nuclear Power Plant

- Two pump experts reached consensus on estimate

- HRA case study on SGTR incident at Indian Point 2 Plant

- Two human factors experts completed SPAR-H worksheets

- Guideline provided novel approach to aggregating estimates 


\section{Regulatory Uses of Guideline}

\section{Goals Met}

- Support probabilistic estimation for hardware and human events for which current models do not provide sufficient detail and for which expert estimation is needed

- Provide scrutable, usable, and streamlined basis for expert estimation in SDP and ASP analyses

- Scrutable: Full documentation through worksheets

- Usable: Analysts able to complete with minimal training; experts able to complete probabilistic estimation using information provided in worksheet

- Streamlined: Full elicitation took a few hours, not days or weeks 


\section{Exercise: Expert Estimation of Cask Handling Error}

- Review the spent fuel handling article in Appendix D

- Using expert estimation, estimate the HEP for HFE 4--Cask Preparation

- The next three slides provide background on a similar task

- Use the Worksheet B of the ASP Expert Elicitation Guideline (see Appendix E) to assist you

- Despite labeling, these worksheets can also be used for HRA, provided you identify likely PSFs

- Report and discuss

- We will aggregate your answers

- NOTE: This is just for fun! In practice, expert estimation isn't quite this easy. This exercise is simply to give you a quick and dirty feel for the process, not to suggest these are valid estimates that could be used in a PRA or HRA. 


\section{Synopsis of Cask Preparation}

Remote or manual cask preparation operations consist of gas sampling, venting, lid unbolting and removal, gas and water cooldown, shield plug unbolting, and attachment of the shield-lug lifting fixture. If the cask contains individual spent nuclear fuel assemblies with no dual-purpose canister, it will be filled with water in the preparation pit and then transferred to the cask unloading pool.

These operations are prior to removal of the spent nuclear fuel assemblies from the transport cask from processing in the waste handling building. If the cask gasses are determined to be contaminated during the sampling process, then the cask is supposed to be transferred to a remediation hot cell for special handling and decontamination. If the cask gasses are not determined to be contaminated, then the cask will remain in the routine processing area, where the spent nuclear fuel assemblies will be removed from the transportation cask and ultimately packaged in a disposal container. 


\section{Personnel with Fuel Pool Access}

\begin{tabular}{|l|l|}
\hline 1. Radiation workers & Handle spent nuclear fuel and other radioactive material \\
\hline 2. Maintenance workers & $\begin{array}{l}\text { Maintain equipment involved in safety-related tasks, such as } \\
\text { hoists, transporters, cranes, and hot-cells }\end{array}$ \\
\hline 3. Nonradiation workers & $\begin{array}{l}\text { Handle excavation, mechanical, and other tasks, but do not } \\
\text { handle radioactive material or equipment for handling } \\
\text { radioactive material }\end{array}$ \\
\hline 4. Managers & $\begin{array}{l}\text { Do not perform operational tasks, but supervise others in } \\
\text { those tasks }\end{array}$ \\
\hline 5. Security & Provide for physical security for the site \\
\hline $\begin{array}{l}\text { 6. Nonsafety facility } \\
\text { workers }\end{array}$ & $\begin{array}{l}\text { Ordinary maintenance workers perform common janitorial and } \\
\text { other routine tasks }\end{array}$ \\
\hline 7. Visitors & $\begin{array}{l}\text { As with the current facility, visitors are expected on a } \\
\text { frequent basis }\end{array}$ \\
\hline
\end{tabular}




\section{Possible Human Errors:}

- The cask vent port is not properly connected to the radiation detector

- The radiation detector is not read correctly to identify contamination, when present

- The cask is not transferred to the remediation hot cell, even though contamination is detected

- A contaminated cask is not properly connected to the exhaust system

- A contaminated cask is not properly purged of contaminated cask gases 


\section{LESSON 7}

HRA Methods Overview 


\section{Lesson 7 Objectives}

- Introduce the most common NRC methods

- THERP

- ASEP

- SPAR-H

- ATHEANA

- Give hands-on in-class examples with THERP and SPAR$\mathrm{H}$

- Continue illustrating key HRA concepts like nominal HEPs and dependence through the examples

- Briefly introduce non-NRC approaches like the EPRI HRA Calculator 


\section{A Snapshot of NRC HRA Methods}

- Technique for Human Error Rate Prediction (THERP)

- Accident Sequence Evaluation Program (ASEP)

- Simplified Plant Analysis Risk-Human Reliability Analysis (SPAR-H) Method

- A Technique for Human Event Analysis (ATHEANA) 


\section{THERP (NUREG-CR/1278)}

- Developed by Alan Swain, et al., at Sandia National Laboratories for US NRC in early 1980s

- Precursors to THERP go back to the 1962

- Parts of what became THERP appeared in WASH-1400

- Based on data gathered from reactor control room, weapons manufacturing, and chemical processing activities, as well as expert estimation

- Historically most widely used method

- Validates as well or better than any other technique 


\section{THERP (Continued)}

- Uses HRA event tree modeling

- Applies data and modifications from tables (see THERP Chapter 20; included here in Appendix F) for quantification

- Often misapplied (quantify top level without modeling and quantifying subtasks) 


\section{How THERP Works}

1. For a given subtask, find the most appropriate lookup table

2. Within the selected lookup table, choose the best fitting Nominal HEP and error factor

3. Modify this value as needed to account for stress, task type, level of experience/training. (Multiply by $1,2,4,5$, or 10—see Table 20-16); yields a Basic HEP

4. Modify this value for dependence, as needed (see Table 20-17); the resulting HEP is called a Conditional HEP 


\section{How THERP Works (Continued)}

5. Calculate values of each failure path.

6. Sum up all failure paths to obtain total task failure.

7. Run sensitivity analysis by making reasonable changes to Nominal, Basic, or Conditional HEPs or by changing model (adding or removing failures and/or recoveries) 


\section{Navigating THERP Tables}

- Figure 20-2 from THERP sorts tables out by their function

- Screening

- Diagnosis

- Errors of Omission

- Errors of Commission

- PSFs

- Uncertainty Bounds

- Recovery Factors 


\section{Navigating THERP Tables (Continued)}

- Figure 20 -1 of THERP Handbook provides overall logic for using THERP and tables

- Pages 20 -11 through 20 -13 of THERP Handbook list all 27 THERP Tables

- Given an HRA Event Tree, to quantify a branch, find the correct table and item 


\section{THERP Dependency}

THERP Definitions:

- Dependency is "Determination of how the probability of failure or success on one task may be related to the failure or success on some other task"

- "Two events are independent if the conditional probability of one event is the same whether or not the other event has occurred. That is, independence is the case in which the probability of success or failure on Task ' $\mathrm{B}$ ' is the same regardless of success or failure on Task 'A'"

- "If events are not independent, they are dependent" 


\section{THERP Dependency (Continued)}

Two types of dependency in THERP

- Direct dependence exists when the outcome of one task directly affects the outcome of a second task

- Failure on Task "A" causes an auditory signal that results in more careful performance on Task " $B$ "

- Failure on Task "A" causes extreme anxiety with a resultant increase in probability of failure on Task "B"

- Failure on Task "A" causes Task "B" to be more difficult with an associated increase in probability of failure 


\section{THERP Dependency (Continued)}

Two types of dependency in THERP (continued)

- Indirect dependence occurs when some PSF or set of PSFs influences the relationship between tasks such that the dependence between them changes

- If the PSF merely raises or lowers the HEPs for tasks without changing the relationship between them, this is not an example of indirect dependence

- A high level of stress tends to increase HEPs across tasks but not necessarily change dependence

- Stress leads to dependency only if it also causes a systematic change in behavior across events (e.g., if stressed operators defer decisions to shift supervisor-something they would not do in an unstressed state) 


\section{THERP (NUREG/CR-1278) Dependency}

THERP covers five levels of dependency, from zero dependence (ZD) to complete dependence (CD)

- Covered for success and failure paths

- Success path = dependency between two events with successful outcomes

- Failure path = dependency between two events with unsuccessful outcomes (human error)

\begin{tabular}{|c|c|c|c|c|}
\hline $\begin{array}{c}\text { Level of } \\
\text { Dependence }\end{array}$ & Success Equations & Equation No. & Fallure Equations & Equation No. \\
\hline ZD & $\operatorname{Pr}\left[s_{N^{*}}\left|s_{m_{N-1}}\right| Z D\right]=n$ & $(10-9)$ & $\operatorname{Pr}\left[F_{N^{*}}\left|F_{m_{N}-1}\right| Z D\right]=N$ & $(10-14)$ \\
\hline LD & $\operatorname{Pr}\left[S_{n_{N}{ }^{n}}\left|S_{n_{N}-1 n}\right| L D\right)=\frac{1+19 n}{20}$ & $(10-10)$ & $\operatorname{Pr}\left[F_{N^{*}}\left|F_{m-1 *}\right| L D\right]=\frac{1+19 N}{20}$ & $(10-15)$ \\
\hline мD & $\operatorname{Pr}\left[s_{n N^{m}}\left|S_{n N-1}\right| M D\right]=\frac{1+6 n}{7}$ & $(10-11)$ & $\operatorname{Pr}\left[F_{m N^{n}}\left|F_{n N-11^{n}}\right| M D\right]=\frac{1+6 N}{7}$ & $(10-16)$ \\
\hline HD & $\operatorname{Pr}\left[s_{N^{\prime \prime}}\left|s_{n^{N}-1^{m}}\right| \mathrm{HD}\right]=\frac{1+n}{2}$ & $(10-12)$ & $\operatorname{Pr}\left(F_{N^{N}}\left|F_{m-1}\right| H D\right)=\frac{1+N}{2}$ & $(10-17)$ \\
\hline CD & $\operatorname{Pr}\left[S_{N^{N N^{m}}}\left|S_{N_{N-1}-1}\right| C D\right]=1.0$ & $(10-13)$ & 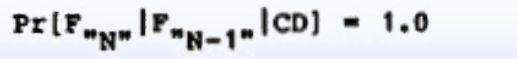 & $(10-18)$ \\
\hline
\end{tabular}




\section{Exercise: THERP Quantification}

- Using the spent fuel handling example, quantify the HFE using THERP values

- Report out and discuss 


\section{ASEP (NUREG-CR/4772), Briefly Noted}

- Developed by Swain as an easy-to-use simplification of THERP

- Provides separate guidance and quantification for preand post-accident tasks

- Distinguishes between screening values and nominal values (those values that are quantified at a more explicit level than the screening values)

- Provides simplified tables according to pre/post accident phase and screening/nominal analysis, with resulting HEP and Error Factors

- Recovery and dependency modeling similar to THERP 


\section{SPAR-H (NUREG/CR-6883)}

The SPAR HRA, or SPAR-H, method was developed to support NRC's ASP program

- The current Standardized Plant Analysis Risk (SPAR) models evolved from the early ASP PRAs

- Now exist in full-power models for each plant

- Being applied to low power and shut down models

SPAR-H is used as a simplified HRA approach

- Like ASEP, SPAR-H is a simplified approach based on THERP

- HEPs in SPAR-H derived from THERP

- Approach uses PSFs instead of sample scenarios, making it easier to generalize 


\section{SPAR-H Quantification}

- SPAR-H Worksheets are used to quantify HEPs by considering factors that may increase/decrease likelihood of error

- Available time

- Complexity

- Procedures

- Fitness for duty

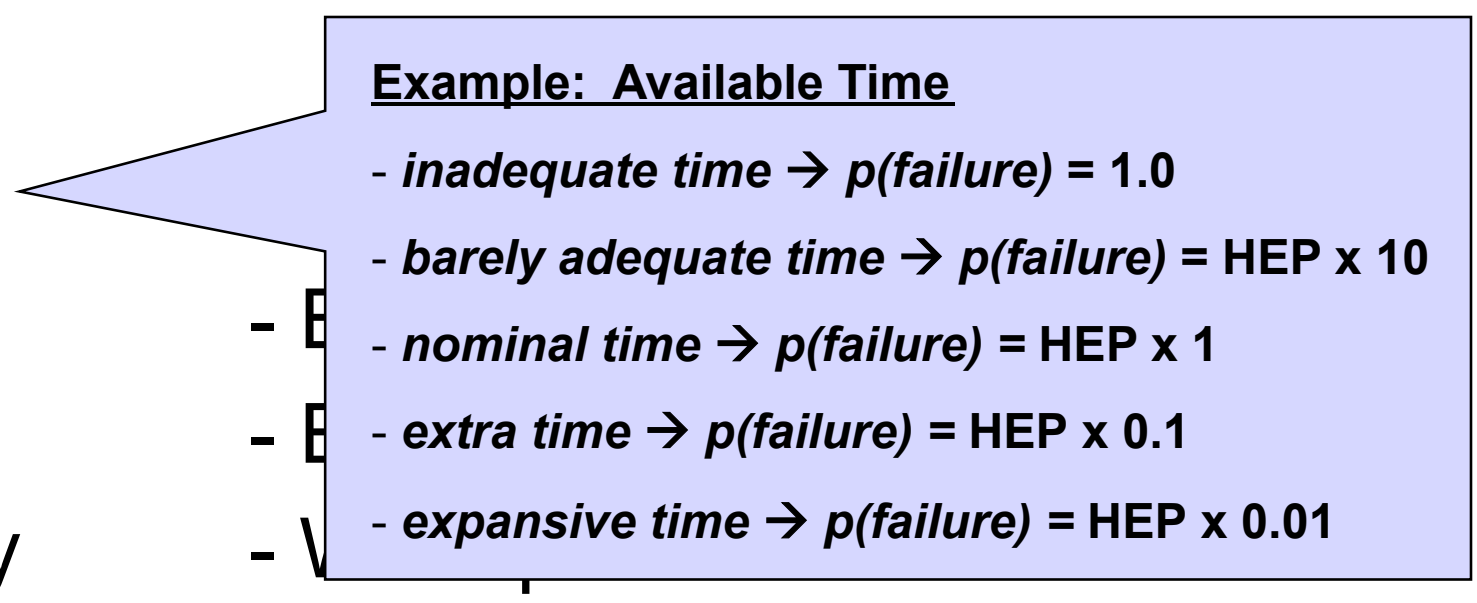

- In the SPAR-H method, these influences are specifically called PSFs 


\section{SPAR-H Shown Graphically}

- PSFs influence performance, which determines likelihood of human error probability

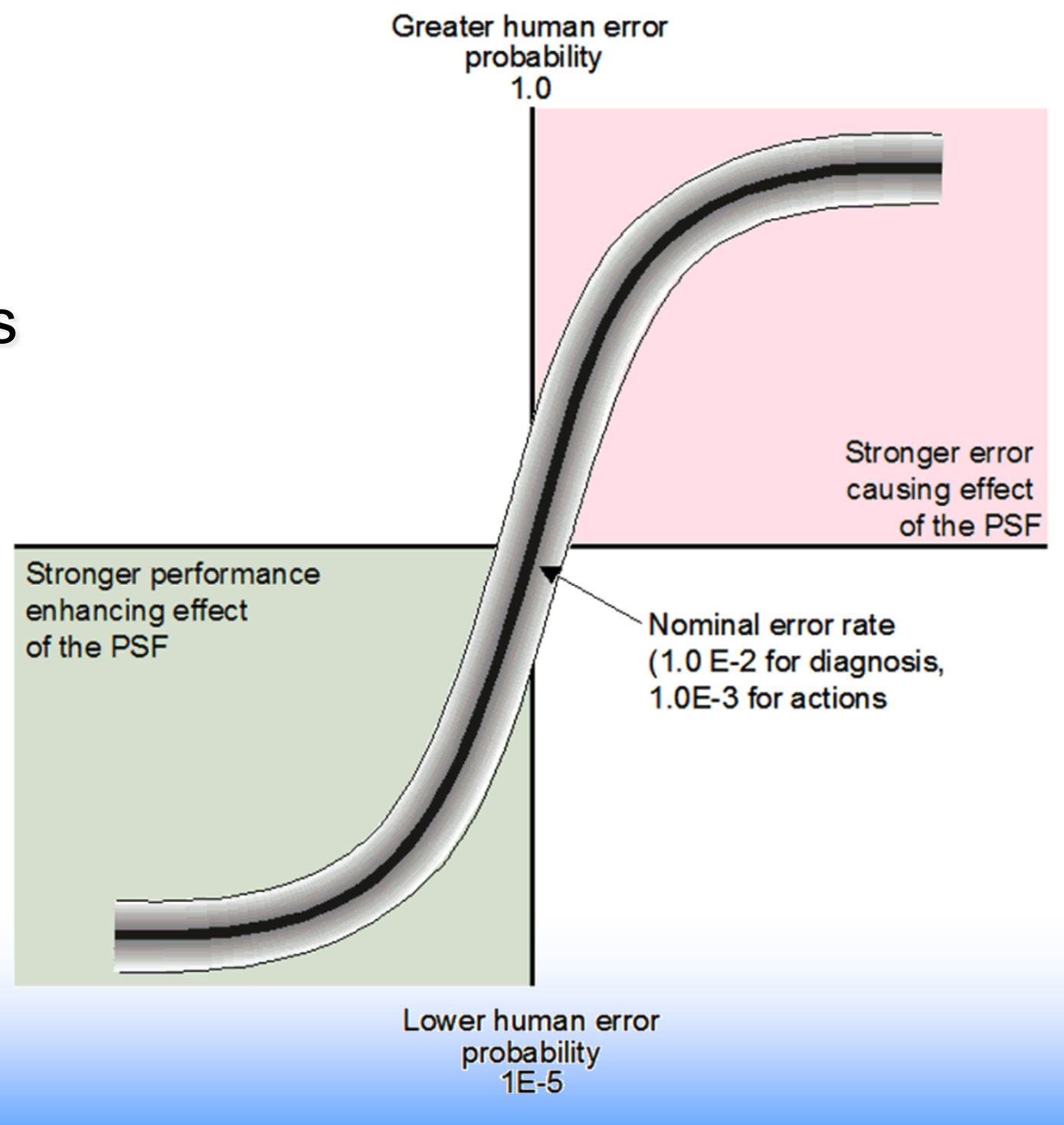
probability 


\section{SPAR-H Worksheet Types}

- The current SPAR-H method has separate worksheets (see Appendix G) for:

- Diagnosis-type activities (e.g., determining whether to start a pump or not)

- Action-type activities (e.g., restoring a pump after it fails, performing a valve line-up)

- Different modes of power operation are included

- At power operations

- Low power and shutdown operations 


\section{SPAR-H Worksheet Process}

- What an example SPAR-H worksheet looks like

- In general, filling out the worksheet follows

Step 1 - Task error ID and question diagnosis

Step 2 - If diagnosis is applicable, complete Table 1

Step 3 - If action is applicable, complete Table 2

Step 4 - Estimate HEP via Table 3 Step 5 - Adjust HEP for dependencies

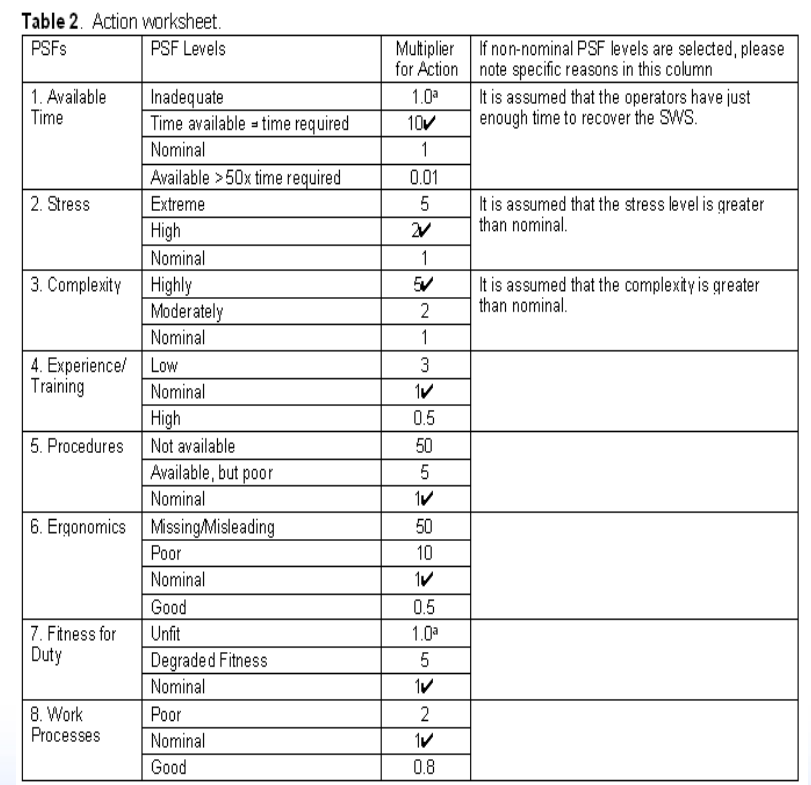




\section{SPAR-H Worksheet Process (cont.)}

Step 4, estimate the HEP:

1. Begin with a "nominal" HEP value

$\supset 1 \mathrm{E}-2$ for diagnosis

$\ni 1 \mathrm{E}-3$ for action

2. Multiply nominal HEP by the applicable PSF "factor"

- For example, if the context related to complexity is "highly complex," PSF factor has a value of 5

- Most factors are greater than one, but some are less than one (this allows for consideration of the positive influence of PSFs which may be present)

3. Repeat step 2 for each PSF 


\section{SPAR-H Worksheet Process (cont.)}

The SPAR-H worksheet allows for efficient estimation of an HEP

- HEP value is assumed to be a mean value

SPAR-H method advocates a "constrained noninformative prior" uncertainty distribution

- This distribution preserves the mean value while expressing relevant uncertainty as a beta distribution

An adjustment factor is provided for instances where multiple, negative PSFs are present

Lastly, dependency between events is considered

- Operator failure on first action implies that subsequent actions may have a higher-than-normal failure probability

- The subsequent SPAR-H HEPs are adjusted upwards in this case 


\section{SPAR-H Dependency Table}

\section{If tasks are dependent, apply the following table:}

Dependency Condition Table

\begin{tabular}{|c|c|c|c|c|c|c|}
\hline $\begin{array}{l}\text { Condition } \\
\text { Number }\end{array}$ & $\begin{array}{c}\text { Crew } \\
\text { (same or } \\
\text { different) }\end{array}$ & $\begin{array}{l}\text { Time } \\
\text { (close in time } \\
\text { or not close } \\
\text { in time) }\end{array}$ & $\begin{array}{l}\text { Location } \\
\text { (same or } \\
\text { different) }\end{array}$ & $\begin{array}{c}\text { Cues } \\
\text { (additional or } \\
\text { no } \\
\text { additional) } \\
\end{array}$ & Dependency & $\begin{array}{c}\text { Number of Human Action Failures Rule } \\
\square \text { - Not Applicable. } \\
\text { Why? }\end{array}$ \\
\hline 2 & \multirow{5}{*}{ s } & \multirow{3}{*}{$\mathrm{c}$} & s & $\mathrm{a}$ & complete & \multirow{10}{*}{$\begin{array}{l}\text { When considering recovery in a series } \\
\text { e.g., } 2^{\text {nd }}, 3^{\text {rd }} \text {, or } 4^{\text {th }} \text { checker }\end{array}$} \\
\hline 3 & & & \multirow[t]{2}{*}{$\mathrm{d}$} & na & high & \\
\hline 4 & & & & $\mathrm{a}$ & high & \\
\hline 7 & & \multirow{2}{*}{ nc } & \multirow[t]{2}{*}{ d } & na & moderate & \\
\hline 8 & & & & $\mathrm{a}$ & low & \\
\hline 9 & \multirow[t]{4}{*}{$\mathrm{d}$} & \multirow[t]{4}{*}{$\mathrm{c}$} & \multirow[t]{2}{*}{$\mathrm{s}$} & na & moderate & \\
\hline 10 & & & & a & moderate & \\
\hline 11 & & & \multirow[t]{2}{*}{ d } & na & moderate & \\
\hline 12 & & & & $\mathrm{a}$ & moderate & \\
\hline 17 & & & & & zero & \\
\hline
\end{tabular}




\section{Class Exercise}

- Example of a medical error in radiation treatment of a patient taken from Set Phasers on Stun by Steven Casey:

- Ray Cox, 33, receiving ninth radiation therapy treatment after removal of cancerous tumor from his shoulder. Everything starting to become fairly routine, and he was quite comfortable with Mary Beth, his radiotherapy technician, and the THERAC-25 radiotheraphy machine. Ray lied face down on table. Mary Beth positioned the THERAC-25 and went into the control room. Mary Beth used a computer terminal to enter commands on THERAC-25. The video and audio between the patient room and the control room were not working. There were two modes: a high-power x-ray dose to radiate tumors and a low-power electron beam for subsequent treatment. Mary Beth accidentally put it in x-ray mode by typing [X] but then corrected it to electron mode by moving the cursor up and typing $[E]$. She then pressed [RETURN] to administer the treatment. 


\section{Class Exercise (Continued)}

- Set Phasers on Stun (Continued):

- No one had every changed an $[X]$ to an [E] before in this manner. Atomic Energy Canada, who developed the THERAC-25, had not anticipated this way of changing the mode. This error not only switched the THERAC-25 into $x$-ray mode, it disabled a metal plate that limited the intensity of the $x$ ray. Ray Cox's intended dose of 200 rads actually became 25,000 rads! Mary Beth activated the first beam but received an error message that sounded like the beam had not been applied. She tried again two more times. The first time, Ray Cox heard a frying sound and felt an excruciating stabbing pain in his shoulder. Rolling in pain, the THERAC-25 fired again, this time into his neck. Screaming in pain, a third dose went through his neck and shoulder. He ran out of the treatment room. Mary Beth, meanwhile, was unaware what had happened, but the THERAC-25 reported Ray had only received 20 rads. In fact, he had received 75,000 rads. Four months later, Ray died due to radiation overdose. He remarked, "They forgot to set the phaser on stun!" 


\section{Class Exercise (Continued)}

- Set Phasers on Stun (Continued):

What Was Supposed to Happen

- Set patient on table

- Position THERAC-25

- Go to control room

- Enter prescribed dose

- Activate dose

- Retrieve patient
What Actually Happened

- Set patient on table

- Position THERAC-25

- Go to control room

- Enter prescribed dose

- Correct wrong entry

- Activate dose

- Error message

- Go back and reactivate

- Error message

- Go back and reactivate

- Patient flees 


\section{Class Exercise (Continued)}

- Set Phasers on Stun (Continued):

What Was Supposed to Happen

- Set patient on table

- Position THERAC-25

- Go to control room

- Enter prescribed dose

- Activate dose

- Retrieve patient

What is the likelihood for entering and giving the wrong dose?
What Actually Happened

- Set patient on table

- Position THERAC-25

- Go to control room

- Enter prescribed dose

- Correct wrong entry

- Activate dose

- Error message

- Go back and reactivate

- Error message

- Go back and reactivate

- Patient flees 


\section{Class Exercise (Continued)}

What is the likelihood for entering and giving the wrong dose?

- First, consider the relevant PSFs from SPAR-H

- Available time

- Complexity

- Procedures

- Fitness for duty
- Stress/stressors

- Experience/training

- Ergonomics/HMI

- Work processes

- Determine which PSFs apply, and which do not 


\section{Class Exercise (Continued)}

- Next, consider if it is a diagnosis (cognitive) or action (behavior)

- Finally, consider the levels of applicable PSFs

- Use the numbers in parentheses on this table

- Calculate the Basic HEP

- Nominal HEP (1E-2 or 1E-3) $x$ Time $x$ Stress $x$ Complexity $x$ Experience $x$ Procedures $x$ Ergonomics $x$ Fitness for Duty $x$ Work Processes

- Correct for too many PSFs

- Adjust for Dependency

\begin{tabular}{|c|c|c|c|}
\hline \multicolumn{4}{|c|}{ SPAR-H (NUREG/CR-6883) } \\
\hline PSFs & PSF Levels & HEP for Diagnosis ${ }^{1}$ & $\begin{array}{c}\text { HEP for } \\
\text { Action }^{1}\end{array}$ \\
\hline \multirow[t]{5}{*}{ Available Time } & $\begin{array}{l}\text { Inadequate time } \\
\end{array}$ & "1.0 (no multiplier) & $\bar{~} 1.0$ (no multiplier) \\
\hline & Barely adequate time & $0.1(10)$ & $0.01(10)$ \\
\hline & Nominal time & $0.01(1)$ & $0.001(1)$ \\
\hline & "'mxtra time & $0.001(0.1)$ & $0.0001(0.1)$ \\
\hline & Expansive time & $0.0001(0.1-0.01)$ & $0.00001(0.01)$ \\
\hline \multirow[t]{3}{*}{ Stress/ Stressors } & Extreme & $0.05(5)$ & $0.005(5)$ \\
\hline & "'High" & $0.02(2)$ & $0.002(2)$ \\
\hline & "Nominal & $0.01(1)$ & $0.001(1)$ \\
\hline \multirow[t]{4}{*}{ Complexity } & Highly complex & $0.05(5)$ & $0.005(5)$ \\
\hline & Moderately complex & $0.02(2)$ & $0.002(2)$ \\
\hline & Nominal & $0.01(1)$ & $0.001(1)$ \\
\hline & Obvious diagnosis & $0.001(0.1)$ & $\mathrm{N} / \mathrm{A}$ \\
\hline \multirow{3}{*}{$\begin{array}{c}\text { Experience/ } \\
\text { Training }\end{array}$} & Low & $0.1(10)$ & $0.003(3)$ \\
\hline & Nominal & $0.01(1)$ & $0.001(1)$ \\
\hline & "'migh" & $0.05(0.5)$ & $0.0005(0.5)$ \\
\hline \multirow[t]{5}{*}{ Procedures } & Not available & $0.5(50)$ & $0.05(50)$ \\
\hline & "Incomplete & $0.2(20)$ & $0.02(20)$ \\
\hline & Available, but poor & $0.05(5)$ & $0.005(5)$ \\
\hline & Nominal " & $0.01(1)$ & $0.001(1)$ \\
\hline & $\begin{array}{l}\text { Diagnostic/symptom } \\
\text { oriented }\end{array}$ & $0.005(0.5)$ & $\mathrm{N} / \mathrm{A}$ \\
\hline \multirow[t]{4}{*}{ Ergonomics/HMI } & $\begin{array}{l}\text { Missing/Misleading } \\
\end{array}$ & $0.5(50)$ & $\overline{0.05(50)}$ \\
\hline & Poor & $0.1(10)$ & $0.01(10)$ \\
\hline & Nominal & $0.01(1)$ & $0.001(1)$ \\
\hline & Good & $0.005(0.5)$ & $0.0005(0.5)$ \\
\hline \multirow[t]{3}{*}{ Fitness for Duty } & Unfit & 1.0 (no multiplier) & 1.0 (no multiplier) \\
\hline & Degraded Fitness & $0.05(5)$ & $0.005(5)$ \\
\hline & "Nominal & $0.01(1)$ & $0.001(1)$ \\
\hline \multirow[t]{3}{*}{ Work Processes } & Poor & $0.02(2)$ & $0.005(5)$ \\
\hline & Nominal & $0.01(1)$ & $0.001(1)$ \\
\hline & Good & $0.008(0.8)$ & $0.0005(0.5)$ \\
\hline
\end{tabular}




\section{Exercise: SPAR-H Quantification}

- Using the spent fuel handling HFE you reviewed earlier, quantify the event using the SPAR-H method

- Assume, for the present purposes, that the "Low Power/Shutdown" worksheets apply, since this is a maintenance task

- Identify if it is action or diagnosis

- Identify the applicable PSFs

- Determine any correction factor or dependency

- Report out and discuss 


\section{ATHEANA (NUREG-1624; NUREG-1880)}

A Technique for Human Event Analysis (ATHEANA)

- Purpose is to "develop an HRA quantification process and PRA modeling interface that can accommodate and represent human performance found in real events"

- Assumption is that HFEs with highly trained staff using considerable procedural guidance "do not usually occur randomly or as a result of simple inadvertent behavior" such as missing a procedure step

- Instead, such HFEs occur when:

- The operator is placed in an unfamiliar situation where training and procedures are inadequate or do not apply

-When some other unusual set of circumstances exists 


\section{ATHEANA Background}

Use of ATHEANA to:

1. Identify plausible error-likely situations and potential errorforcing contexts

\section{Error forcing contexts (EFCs)}

- $\quad$ arise when combinations of PSFs and plant conditions create an environment in which unsafe actions are more likely to occur-a situation that is setting up the operator to "fail"

Unsafe actions (UAs)

- $\quad$ are actions taken inappropriately or not taken when needed that result in degraded safety; unsafe actions don't necessarily lead to an error

2. Define HFEs pertinent to performing human actions incorrectly

3. Determine HEPs 


\section{ATHEANA Background (Continued)}

\section{Unique Features of ATHEANA}

1. Identify operational vulnerabilities the could set up UAs

- E.g., procedure weaknesses

2. Identify plausible deviations from nominal scenarios

\section{Nominal scenario}

- The expected or representative case scenario included in the PRA

3. Identify important PSFs relevant to both nominal and deviation scenarios

4. Identify other factors that could significantly affect the likelihood of the HFEs 


\section{ATHEANA Background (Continued)}

\section{When to Use ATHEANA}

- Use ATHEANA if risk-informed decision making requires:

1. Understanding vulnerabilities associated with specific UAs instead of generic HFEs

- E.g., submittal that includes procedural change

2. Understanding the contexts of specific EFCs (rather than a generic scenario context)

- E.g., need for a more detailed HRA as part of a PRA

3. Understanding a wide range of PSFs under different contexts and scenarios

- E.g., screening analysis reveals particular HFEs that are risk significant, and it is desired to have a thorough analysis of those HFEs 


\section{Steps of ATHEANA}

\section{Steps synthesize much of}

what has been covered in this course:

- Identifying errors

- Modeling errors in the PRA

- Quantify the errors using expert elicitation

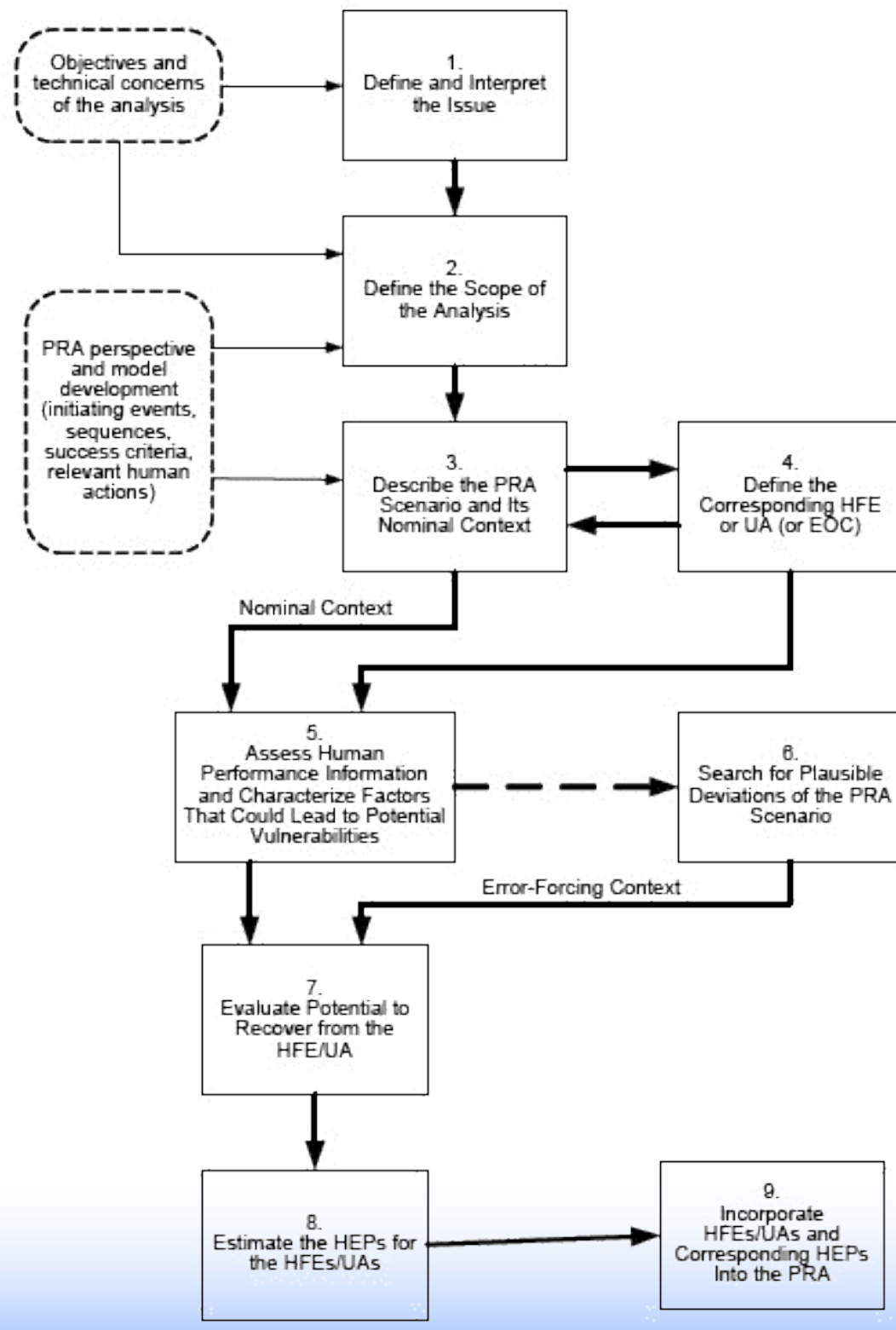




\section{Steps of ATHEANA (Continued)}

Step 1: Define and Interpret Issue

- Assemble ATHEANA team

- HRA analyst

- PRA analyst

- Operations expert

- Operations personnel

- Get background information

- Identify audience to whom the issue resolution is to be provided

- Define the issue in HRA terms

- Provide an overall risk framework for resolving the issue 


\section{Steps of ATHEANA (Continued)}

Step 2: Define Scope of Analysis

- Prioritize what is necessary

Step 3: Describe the Nominal Context

Step 4: Define the Corresponding HFE or UA

- Identify the human actions (HFE/UA) for the PRA

Step 5: Assess Potential Vulnerabilities

- Consider the time phases (e.g., pre-/post- initiator) for the analysis

- Review influence of PSFs

- PSF weights may vary from one context to another 


\section{Steps of ATHEANA (Continued)}

\section{Step 6: Search for Plausible Deviations from PRA Scenario}

- Consider scenarios that can cause operators problems in detecting, understanding, or responding to situation

\begin{tabular}{|l|l|}
\hline $\begin{array}{l}\text { Garden path problems (Strong } \\
\text { but incorrect evidence) }\end{array}$ & $\begin{array}{l}\text { Changing situations (Failure to } \\
\text { notice new conditions) }\end{array}$ \\
\hline Missing information & Misleading information \\
\hline $\begin{array}{l}\text { Masking activities (Other } \\
\text { activities may hide underlying } \\
\text { problem) }\end{array}$ & $\begin{array}{l}\text { Multiple lines of reasoning } \\
\text { (Conflicting strategies) }\end{array}$ \\
\hline Side effects & Impasse \\
\hline Late changes in plan & $\begin{array}{l}\text { Dilemmas (Ambiguity causes } \\
\text { doubt about appropriate } \\
\text { action) }\end{array}$ \\
\hline Trade-offs & $\begin{array}{l}\text { Double binds (Two undesirable } \\
\text { elements) }\end{array}$ \\
\hline $\begin{array}{l}\text { High tempo, multiple tasks } \\
\text { (Operator overload) }\end{array}$ & Need to shift focus of attention \\
\hline
\end{tabular}




\section{Steps of ATHEANA (Continued)}

Step 6: Search for Plausible Deviations from PRA Scenario (Continued)

- Screen out deviations that are not risk significant Step 7: Evaluate Potential for Recovery Step 8: Estimate the HEPs for the HFEs/UAs

- Use guided expert estimation approach with facilitator and panel of experts

Step 9: Incorporate HFE/UA and HEP into PRA 


\section{Other HRA Methods}

- As noted earlier, there are over 40 HRA methods

- THERP, ASEP, and SPAR-H are the most common in use by the NRC

- Additional methods you may encounter from industry include

- EPRI HRA Calculator

- Or any of over 50 HRA methods 


\section{EPRI HRA Calculator}

- Software tool to combine several HRA methods for quantifying pre- and post-initiator HFEs

- Includes

- EPRI Cause-Based Decision Tree Method (CBDTM)

- Human Cognitive Reliability/Operator Reactor Experiments (HCR/ORE)

- ASEP

- THERP

- SPAR-H

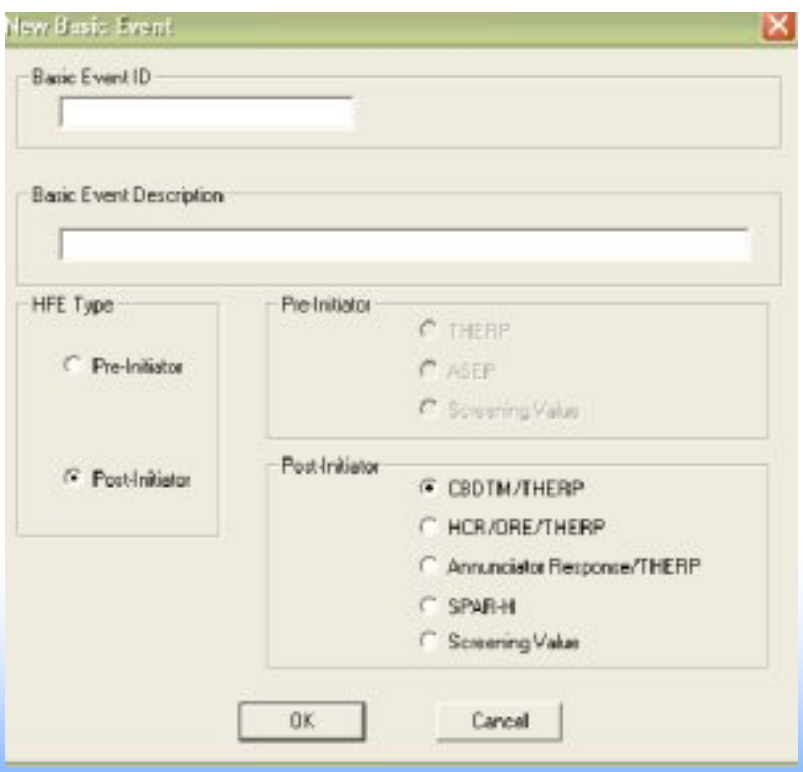




\section{EPRI HRA Calculator (Continued)}

HCR/ORE Implementation:

- Linked to EPRI ORE data collection

- Control room operator actions

- Emergency and abnormal operating procedures based

- Similar to operator action tree approach

- Recognizes a time window exists for which functions must be completed

- Task decomposition required

- Nominal screening curve provided based on normalized time reliability curve

- Operator/crew performance influenced by cues and responses as indicated in procedures 


\section{EPRI Calculator (Continued)}

CBDTM Implementation:

- Number of decision trees provided:

- Data not available

- Indication not available, inaccurate, warning not present in procedures, training on indicators not provided,

- Data not attended to

- Workload, one-time check versus continuous, front versus back panel, alarmed versus not alarmed

- Data misread

- Indicators not easy to locate, human engineering deficiencies, formal communications protocols present/ not present,

- Information misleading

- Are cues in procedures, indicator obviously failed, procedures warn of differences, specific training

- Probability of crew response is adjusted for recovery. 


\section{Choosing Between Methods}

Advantages of Each NRC Method

- Full Qualitative Analysis

- THERP, ATHEANA

- Simplicity of Estimation Process (Screening Tool)

- ASEP, SPAR-H

- Flexibility to Cover Unusual Events

- ATHEANA

- Coverage of Cognitive Factors

- SPAR-H, ATHEANA

- Complete Method (Identification, Modeling, Quantification)

- THERP, ATHEANA

Remember, there are over 60 HRA methods that may meet particular applications beyond what has been described here

- Distilling the most useful methods for particular applications is task of ongoing NRC projects under Dr. Erasmia Lois

- Shift from developing new methods to validating existing methods 


\section{HRA Good Practices (NUREG-1792)}

- Two main purposes:

- Guidance for performing HRAs

- Support the review of HRAs

- Not method specific

- Method comparisons found in NUREG-1842

- For reactor, full power, internal events

- Supports RG 1.200

- For reference, both NUREG-1792 and NUREG-1842 found on course CD 


\section{Good Practices for HRA in of PRA}

Specifies the HFEs modeled in PRAs that are associated with normal operations including:

- Events leaving equipment in an unrevealed, unavailable state

- Those that induce an initiating event

Specifies the HFEs modeled in PRAs associated with emergency operation including:

- Events that, if not performed, do not allow a desired function to be achieved or recovered 


\section{Good Practices PSFs}

\section{Recall the following PSFs:}

\begin{tabular}{|c|}
\hline $\begin{array}{c}\text { Good Practices PSFs } \\
\text { (NUREG-1792) }\end{array}$ \\
Training and Experience \\
\hline Procedures and Administrative Controls \\
\hline Instrumentation \\
\hline Time Available \\
\hline Complexity \\
\hline Workload/Time Pressure/Stress \\
\hline Team/Crew dynamics \\
\hline Available Staffing \\
\hline Human-System Interface \\
\hline Environment \\
\hline Accessibility/Operability of Equipment \\
\hline Need for Special Tools \\
\hline Communications \\
\hline Special Fitness Needs \\
\hline Consideration of 'Realistic' Accident \\
Sequence Diversions and Deviations \\
\hline
\end{tabular}




\section{Good Practices in a Nutshell}

What to Look for in Identification and Modeling

- Include human actions as part of overall PRA

- Include all necessary and expected activities

- Include backup actions to failed automatics

- Include procedure driven or skill of the craft recovery

What to Look for in Quantification

- Include screening and full analysis for risk-significant activities

- Consider PSFs, dependency, and uncertainty 
APPENDIX A 


\section{The Fallible Engineer}

Australian engineers feel that they are being blamed for accidents and failures that are beyond their control. They want the public to understand that experts are only human.

Sharon Beder

At four o'clock in the morning of 30 April 1988, a railway embankment near the coastal town of Coledale in New South Wales collapsed, sending tons of mud and water down a hill. The debris crushed a house, killing a woman and child who were inside. The area was prone to subsidence and evidence given at the inquest suggested that the designers of the embankment had not taken proper account of this. Four people, two of them engineers, were subsequently charged with endangering passengers on a railway. One, a principal geotechnical engineer with the State Rail Authority of New South Wales, was also charged with two counts of manslaughter.

Though none of them was convicted, the engineering profession was horrified that engineers should be charged in this way, and rallied to their support. Peter Miller, chairman of the standing committee on legal liability of the Institution of Engineers, Australia, argued that criminal prosecutions against engineers set a precedent that could change the way engineering was practiced. He said it was likely to result in engineers becoming more conservative in their assessments and decisions. Although this was not in itself a bad thing, it would mean higher costs for engineering work, he claimed.

The institution was also concerned about individual blame being apportioned to engineers who work as part of a team in organizations operating under financial constraints. Bill Rourke, who retired last month as the institution's chief executive, pointed out in its magazine, Engineers Australia, that safety margins are closely related to the availability of funds. He argued that the provider of those funds, in this case the community, should carry a significant responsibility for safety levels.

The issue of who should take responsibility when things go wrong is becoming a central concern for the engineering profession worldwide. At the end of last year the Australian institution sent all its members a discussion paper entitled Are you at risk? Managing Expectations. More than 3000 engineers replied, the largest response the institution has ever had on any issue. In the preface to the paper, the institution's president, Mike Sargent, said that the trend towards criminal prosecutions for negligence and escalation of civil law claims against engineers "constitute a significant threat to the ability of our profession to serve the community and might even threaten its continued existence."

Miller, too, believes that the profession is at risk. "Engineers are being put in untenable positions," he says. "they are being asked to make decisions over matters they cannot control and being forced to take responsibility for these decisions." What Miller and his colleagues at the Institution of Engineers are proposing is nothing short of a radical change in the relationship between engineer and society. The engineering profession seems to be approaching a turning point. 
Miller and his colleagues believe that if people are more aware of the uncertainties surrounding engineering work and the limitations of mathematical models, then they would not so readily blame engineers for failures. The institution's discussion paper pointed out that engineers had presented a falsely optimistic and idealistic view of their work. They are now paying the price for having raised unjustifiably high the public's expectations of what they can deliver. "We know (or should know) that our models are limited as to their ability to represent real systems, and we use (or should use) them accordingly. The trouble is that we are so inordinately proud of them that we do not present their limitations to the community, and leave the community with the impression that the models are precise and comprehensive."

The discussion paper quotes the 1946 chairman of the Scottish branch of Britain's Institution of Structural Engineers as saying: "Structural engineering is the art of modeling materials we do not wholly understand into shapes we cannot precisely analyse so as to withstand forces we cannot properly assess in such a way that the public at large has no reason to suspect the extent of our ignorance."

Why have engineers misled the public in this way? Gavan McDonnell, an engineer and supervisor of the graduate program in science and society at the University of New South Wales, says: "It is the very nature of professions to fill the role of a sort of priesthood with transcendental access to superior knowledge. Engineers have assumed this role, too. They have protected their professional status as possessors of special knowledge and have not been inclined to discuss the limitations of that knowledge with those outside the profession." McDonnell admits that there is a large element of technocratic arrogance in this stance, but says that modern societies require this division of knowledge in order to function. There is, however, an important rider: "Previously the community trusted in the probity and ethical rightness of the expert," he says. "But as experts are increasingly seen to be working for particular interests in society, that trust is disappearing."

Miller, too, points to the breakdown of the social contract between engineers and society. He says that the contract involved a commitment by engineers to always put the public interest first and a commitment by the public to allow engineers to regulate themselves. "That contract is now seen to be broken by both parties," he says. The institution's discussion paper is the first step in a process of re-establishing trust between engineers and the public. Miller, one of the authors of the paper, was at first hesitant about sending it out. He was worried that engineers might not be interested in questions that don't have clear-cut answers, and concerned that they would not want to discus philosophy-even engineering philosophy. He has been gratified to find an unsuspected hunger for such a discussion.

The philosophy set out in the paper is that engineering is an art rather than a science, and as such depends heavily on judgment. The widespread use in engineering of heuristics, or "rules of the thumb," requires judgment to be used properly. Billy Vaughn Koen, professor of mechanical engineering at the University of Texas at Austin, defines a heuristic device as "anything that provides a plausible aid or direction in the solution of a problem but is in the final analysis unjustified, incapable of justification and infallible." Heuristics is used in the absence of better knowledge or as a short-cut method of working out something that would be too expensive or too time-consuming to work out more scientifically. 
An example of a heuristic device is a "factor of safety," sometimes referred to as a "factor of ignorance." Engineers have to work with materials that vary widely in strength and other characteristics, and design for a range of operating conditions and loads. To cope with these variations and uncertainties they employ factors of safety. Henry Petroski, an American engineer who has written extensively on engineer accidents, explains: "Factors of safety are intended to allow for the bridge built of the weakest imaginable batch of steel to stand up under the heaviest imaginable truck going over the largest imaginable pothole and bouncing across the roadway in a storm."

However, the concept of a factor of safety is often misunderstood by those outside the profession as implying some large safety margin on a predictable design. Barry McMahon, a Sydney-based geotechnical engineer, has found his clients believe that as factor of safety implies "certainty" plus a bit more. He says they are far more concerned with the financial risk of "conservative" design (design that errs on the safe side) than they are with other sources of risk. Conservative design tends to be more expensive, which means that there is always pressure to reduce factors of safety. For a factor of safety to be effective, the means of failure must be known and the cause of the failure determinable by experiment. For example concrete columns may be designed to cope with 10 times the compression stresses the engineer estimates they will have to bear. In this case the factor of safety is 10. But this assumes that if the columns are going to fail it will be as a result of compression.

If the columns are subject to unexpected forces from another direction-so that they are stretched instead of compressed, for example - then their extra ability to take compression will not be of much help. The ability of a concrete column to bear a particular stress is determined by experiments done repeatedly on concrete columns in the laboratory.

All engineering structures incorporate factors of safety and yet some still fail, and when this happens the factor of safety for similar structures built subsequently might be increased. Conversely, when a particular type of structure has been used often without failure, there is a tendency for engineers to suspect that these structures are overdesigned and that the factor of safety can be reduced. Petroski says: "The dynamics of raising the factor of safety in the wake of accidents and lowering it in the absence of accidents can clearly lead to cyclic occurrences of structural failures." He points out that this cyclic behaviour occurred with suspension bridges following the failure of the Tacoma Narrows Bridge, which collapsed spectacularly in 1940 in mild winds.

Cutting safety margins to reduce costs in the face of success happens in all engineering disciplines. William Starbuck and Frances Milliken, researchers at New York University, have studied the catastrophic failure of the challenger space shuttle in January 1986 and concluded in their paper "Challenger: fine-tuning the odds until something breaks" (Journal of Management Studies, Vol. 25, July 1988) that the same phenomenon was present there. They argue that, as successful launches accumulated, the engineering managers at NASA and Thiokol, the firm responsible for designing and building the rocket boosters for the shuttle, grew more confident of future successes. NASA relaxed its safety procedures, treating the shuttle as an "operational" 
technology rather than a risky experiment, and no longer tested or inspected as thoroughly as they had the early launches.

\section{Signs of Failure}

The O-rings sealing the joints in the shuttle's solid-fuel rocket booster, which were eventually found to have played a major role in the accident ("Why Challenger Failed," New Scientist, 11 September 1986), had shown signs of failure in after three of the five flights during 1984 and after eight of nine flights during 1985. But since this damage had not impeded the shuttle launch, engineering managers at NASA and Thiokol came to accept this damage as "allowable erosion" and "acceptable risk." Lawrence Mulloy, manager of the solid rocket booster project, is quoted by Starbuck and Milliken as saying: "Since the risk on O-ring erosion was accepted and indeed expected, it was no longer considered an anomaly to be resolved before the next flight."

Brian Wynne, a researcher at the University of Lancaster, has also studied the Challenger disaster and other accidents. He says that O-ring damage and leakage had come to be accepted as "the new normality." Wynne argues that implementing designs and operating technological systems involve "the continual invention and negotiation of new rules and relationship" and that if this did not happen most technological systems would come to a halt. Starbuck and Milliken agree with respect to the space shuttle. They point out that NASA had identified nearly 300 special "hazards" associated with the launch of Challenger. "But if NASA's managers had viewed these hazards so seriously that any one of them could readily block a launch, NASA might never have launched any shuttles."

Wynne says there is a tendency to refer to "human error" when accidents occur, as if there has been some "drastic departure from normal rule-bound operating practices, and as if we were exonerating a supposedly separate mechanical, nonsocial part of the system." He suggests that part of the problem may be that technological systems are designed as if organizations can operate with perfect communication and that people are not prone to distraction, illogic or complacency. Jean Cross, professor of safety science at the University of New South Wales, agrees that engineers have a tendency to neglect what she calls the "human/technology interface" in their designs. For example, they do not take account of how long it takes people to process information and how people behave when they are under stress.

The institution's paper gives some recognition to this. It says that the notional probability of failure implicit in engineering codes does not give sufficient weight to human factors. "It deals mainly with those issues for which we can rationally compute factors of safety." Miller is keen for engineers to give more consideration to the human/technology interface. This is one of the areas that will be covered in a second discussion paper, which is being put together at the moment.

For Starbuck, Milliken, Wynne, Petroski and many others, all engineering design involves experimentation. According to Petroski, "each novel structural concept - be it a sky walk over a hotel lobby, a suspension bridge over a river, or a jumbo jet capable of flying across the oceans - is the hypothesis to be tested first on paper and possibly in the laboratory but ultimately 
to be justified by its performance of its function without failure." Failures will occasionally occur. They are unavoidable, he argues, unless innovation is completely abandoned.

Wynne goes further, arguing that the experimental nature of engineering extends beyond the designing stage: "If technology involves making up rules and relationships as its practitioners go along, it is a form of social experiment on the grand scale." Similarly, Starbuck and Milliken say that "fine tuning is real-life experimentation in the face of uncertainty."

If engineering is based on incomplete models and on judgment and experimentation, who should be held responsible when engineering projects fail, causing loss of life and property, and damage to the environment? For many engineers this is not a useful question. Mark Tweeddale, professor of risk engineering at the University of Sydney, argues that finding who is to blame for an accident is a fruitless way of going about things. "If someone makes a mistake, you need to ask what caused them to make that mistake? Was it the stress they were under? Was it that they were not properly trained? Should they never have been hired for the job? All these questions lead back to management, but management is also human and the same questions apply. It's like peeling an onion: in the end you are left with nothing." This does not mean an accident shouldn't be investigated. But Tweeddale feels that legal proceedings to establish blame are unhelpful in sorting out the lessons to be learnt from an accident, because the sub judice laws that come into play during a court case restrict free and open public discussion of what happened.

Engineers feel that the public is increasingly looking for someone to blame when accidents happen, rather than accepting accidents as an inevitable part of life. They are frustrated at what seems to be the public's requirement for complete safety. Simon Schubach, a consulting engineer who does risk assessments for the New South Wales planning department, is often asked at public meetings: "Will it be safe?" But the audience seldom accepts his answer, which tends to be along the lines of: "On the basis of the assumptions we made, and the limited applicability of the models we used, our assessment is that the project will meet acceptable risk criteria." Schubach finds the public's demand for certainty naïve, unreasonable, and ill-founded: "Engineering is just not like that."

McDonnell is also concerned about the increasing tendency for lawyers to look for someone to hold liable whenever anything undesirable happens after engineers have given advice. However, he argues that the law still has a part to play where there has been gross negligence and dereliction of duty. This may mean criminal prosecutions of engineers in some instances," he says. "Engineers simply can't expect to be immune from this."

Australia's Society for Social Responsibility in Engineering believes that engineers should accept responsibility for safety of their work even if this means they will be held criminally liable. Philip Thornton, president of the society, says: "If an engineer makes a structure stronger because the risk of being charged if that structure collapses is too high, then the risk of someone being killed or injured is also too high." Thornton argues that if engineers are concerned about being personally liable for accidents and failures then they are less likely to bow to economic pressure to reduce safety margin. "Caution is a good thing." 
The dilemma for engineers today is how to tell the public of the extent of their ignorance without losing the community's confidence. Getting public acceptance of new or controversial technologies is greatly assisted by portraying them as perfectly predictable and controllable. "Concern for public reassurance produces artificially purified public accounts of scientific and technological methods and processes," says Wynne. "When something goes wrong, this background is an ever more difficult framework against which to explain that even when people act competently and responsibly, unexpected things can happen and things go wrong."

The emerging recognition that this situation cannot go on is leading Australian engineers to question their role as "problem solver" who design projects and advocate them as the "right" solutions to community problems. The Institution of Engineers is suggesting a shift to a different role for engineers as "technical advisers" who put forward options for the community to choose from. This means forgoing some of their autonomy and status as technological decision makers in favor of sharing the decisions, in order to share the responsibility of things go wrong. McDonnell argues that the social contract between engineers and the community will not disintegrate if ways can be developed of consulting the public and allowing the community to monitor and vet projects.

It will not be easy for people like Miller and his like-minded colleagues in the Institution of Engineers to bring engineers around to this sharing of responsibility and decision making, and to open and frank dialogue with the community. The change will require a lot more discussion within the profession and changes in engineering education and perhaps public education. Yet Miller is heartened by the overwhelmingly positive response he has had from engineers in Australia.

Sharon Beder is a member of the Institution of Engineers, Australia, and of the Society for Social Responsibility in Engineering. She is currently environmental education coordinator at the University of Sydney.

Tom Wyatt is read in structural design in the Department of Civil Engineering at Imperial College, London.

Further reading: Are you at Risk? Managing Expectations. Institution of Engineers, Australia, 1990; Henry Petroski, To Engineer is Human: The Role of failure in Successful Design, MacMillan 1985; Brian Wynne, "Unruly technology: Practical rules, impractical discourses and public understanding," Social Studies of Science, Vol 18, 1988; William Starbuck and Frances Milliken, "Chalenger: fine-tuning the odds until something breaks," Journal of Management Studies, Vol 25, July 1988. 
APPENDIX B 


\section{Event A Description (from NUREG/CR-6088)}

Event A involved the high dose rate (HDR) remote brachytherapy treatment modality and was categorized as a misadministration involving delivery of a dose to the wrong site.

Description of the event. On the afternoon of November 27, 1991, the day before Thanksgiving holiday, a male patient scheduled to receive his fifth and final radiation therapy treatment for cancer of the nasal septum was placed in the HDR treatment room. A catheter was attached to the patient's nose. A trained resident physician attached this catheter to the HDR unit. When the patient was ready to be treated, a physicist was paged to operate the unit. The physicist who operated the HDR unit during this particular patient's first four treatments was not available. A second authorized physicist proceeded to the treatment area where he picked up a patient's chart located to the left of the HDR console and programmed the unit's computer with the treatment card taken from the chart. Entry of the information from the treatment card into the unit's console produced a printout of the treatment parameters (source dwell times and positions). The HDR unit was activated after the physicist and the resident physician verified that the treatment parameters on the chart corresponded with those on the printout. As the treatment began, one of the three observers standing near the console inquired about the length of the treatment. The resident physician indicated that the treatment would last about one and one half minutes, whereas the physicist indicated a time greater than 400 seconds. Based on this disparity, the resident physician reviewed the chart and discovered that it did not belong to the patient being treated. The appropriate patient chart had been placed to the right of the console. The unit was reprogrammed with the correct information and the treatment progressed normally.

Consequences of the Misadministration. As a result of using the wrong treatment parameters, the licensee reported that the patient's lips received an unintended dose of 76 cGy. As of the date of the team visit, the licensee reported that the patient had not exhibited any adverse aftereffects as a result of the misadministration. 


\section{Event G Description (from NUREG/CR-6088)}

Description of the Event. On November 16, 1992, an 82-year-old female patient was undergoing radiation therapy for an anal carcinoma. The radiation therapy was to be administered by a HDR afterloader with five connecting catheters. For that day's treatment, a dose of $6 \mathrm{~Gy}$ (600 rad) was to be administered through five catheters implanted as a single-plane perineal (rectal) implant encompassing the tumor. After a trial run through the five catheters with a dummy source, the Ir-192 source was easily placed in four of the five catheters. After several unsuccessful attempts to insert the source into the fifth catheter, the physician directed termination of the treatment. An area radiation monitor in the treatment room was observed in an alarm condition-flashing red light — at some point during the unsuccessful attempts to insert the source into the fifth catheter. Although three technologists and the physician were aware of the alarm, no one used the available portable survey meter to detect whether radioactivity was present. Believing that the area radiation monitor was malfunctioning, they reset the area radiation monitor and returned the patient to a local nursing home without performing any radiological surveys. The staff were unaware that the Ir-192 source had remained in the patient.

The patient was returned to the nursing home where she resided with four of the original five treatment catheters, one containing the Ir-192 source, in place. One loose catheter had been removed at the clinic. The source remained in the patient's body for almost four days. On the fourth day, the catheter with the source came loose, and early on the morning of November 20, 1992 the catheter fell out. The patient died on November 21, 1992.

Consequences of the Misadministration. The NRC's medical consultant determined that the radiation the patient received from the Ir-192 source was a probable contributing cause of her death. 
APPENDIX C 


\title{
Human Factor Analysis of JCO Criticality Accident
}

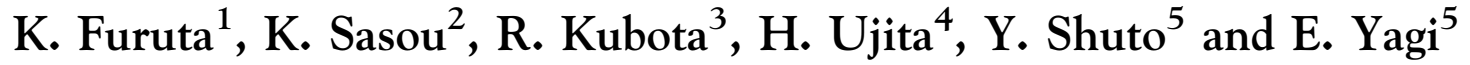 \\ ${ }^{1}$ Institute of Environmental Studies, University of Tokyo, Tokyo, Japan; ${ }^{2}$ Human Factors Research Centre, Central \\ Research Institute of Electric Power Industry (CRIEPI), Tokyo, Japan; ${ }^{3}$ Hitachi Works, Hitachi Ltd, Hitachi, Japan; \\ ${ }^{4}$ Advanced Simulation Systems Department and Institute of Human Factors, Nuclear Power Engineering Corporation \\ (NUPEC), Tokyo, Japan; ${ }^{5}$ Human Factors Laboratory, Research Institute for Social Safety (RISS), Tokyo, Japan
}

\begin{abstract}
The criticality accident that occurred on September 30, 1999 at a uranium processing plant in Tokai-mura was an unprecedented nuclear accident in Japan, not only because it caused deaths of two workers due to radiation casualty but also because it called for evacuation and sheltering indoors to nearby residents. The accident was not directly caused by failures or malfunctions of hardware but by workers' unsafe action deviated from the approved procedure. It was a typical organizational accident in that several organizational factors worked behind. This article is to analyze various causal factors that lead to the accident, including situational factors of workers' unsafe action that triggered the accident, operational and business management of the company, and nuclear safety regulation by the government. It also discusses problems of emergency response after the accident.
\end{abstract}

Keywords: Criticality accident; Human factors; Organizational accident; Safety regulation; Emergency response; Safety culture

\section{INTRODUCTION}

A criticality accident occurred on 30 September 1999 at a uranium-processing plant operated by JCO Co. Ltd (hereafter referred to as JCO) in Tokai-mura, Ibaraki Prefecture, Japan. The accident was not only the first criticality accident, but also an unprecedented nuclear accident in Japan in that it caused the death of two JCO workers by radiation, and that it called for evacuation within a $350 \mathrm{~m}$ radius and sheltering indoors within a $10 \mathrm{~km}$ radius of the JCO plant.

The media reported soon after the accident that workers' unsafe action deviating from the approved procedure had caused the accident rather than failures or malfunctions of hardware. Several organisational factors of JCO and the regulatory body behind the accident were also reported, and it was clear that the accident was a typical organisational accident. Interested in these points, the Division of Human-Machine Systems Studies, the Atomic Energy Society of Japan, organised a special task force to investigate the accident primarily from the viewpoint of human factors. This article reports the result of this investigation and discusses the causal factors of the accident and lessons learned.

The investigation, which was carried out on a voluntary and unofficial basis, could not help relying primarily on open materials presented to the Accident Investigation Committee (AIC) under the Nuclear Safety Commission and those reported in the media. We tried, however, to look into the accident from the academic viewpoint to acquire maximum lessons, apart from prosecution by the officials and the public. It should be mentioned clearly here that participation in the task force was voluntary and the opinions and ideas presented in this article do not necessarily represent those of the organisations to which the authors are affiliated.

In this article, after an overview of the accident has been given, we will first discuss the situational factors that facilitated the workers' unsafe action, and then organisational factors of JCO, particularly problems in operational management and business management. In addition to the organisational factors of JCO, those of the regulatory body that overlooked JCO's violations of its own safety rule will be focused on. Since it has been pointed out that an inappropriate response to the accident delayed the termination and enlarged the impact of the accident, we will look into problems in accident management by JCO, the central government and the local governments. Finally, we will conclude the article by summarising the causes of the accident and the lessons learned. 


\section{JCO AND ACCIDENT SUMMARY}

\subsection{About JCO}

JCO (a subsidiary of Sumitomo Metal Mining Co. Ltd) is a nuclear fuel-processing company with the head office in Tokyo and one plant site in Tokai-mura (AIC Final Report, 27 December 1999). 110 people were working for $\mathrm{JCO}$ as of September 1999. Its capital was $¥ 1$ billion (about \$10 million) and its sales in 1998 fiscal year, which ended March 1999, was $¥ 1723$ million (about $\$ 17$ million). Its main products are purified $\mathrm{UO}_{2}$ powder for light water and experimental fast breeder reactors, and $\mathrm{U}_{3} \mathrm{O}_{8}$ solution for the experimental fast breeder reactor.

JCO operates three processing facilities in Tokai-mura (see Fig. 1). Two of them in the first and second fabrication facilities are for low enriched uranium (less than $5 \%$ of enrichment), with annual capacity of 220 and 495 tons respectively. The third in the conversion building located at the western side of the site is for moderately enriched uranium with enrichment of not more than $50 \%$. Its annual capacity is 3 tons.

The equipment in the conversion building was originally designed for making $\mathrm{UO}_{2}$ powder of $12 \%$ enrichment from uranium hexafluoride $\left(\mathrm{UF}_{6}\right)$, uranium yellow cake or scrap (see Fig. 2). All equipment without the precipitation tank was designed with criticality safe geometry. The shape of the precipitation tank influences crystal formation of $\left(\mathrm{NH}_{4}\right)_{2} \mathrm{U}_{2} \mathrm{O}_{7}$ so that it was impossible to be designed with criticality safety (AIC document no. 8-4, 4 December 1999). Therefore, two licence conditions were made by the regulatory authority. The first is mass control in the precipitation tank. Each batch to be handled in the precipitation tank is limited, depending on the enrichment of uranium; $2.4 \mathrm{~kg}$ of uranium is a batch for uranium with an enrichment of $16-20 \%$. The second is a batch control to limit handling to only one batch of uranium in the equipment until the precipitation tank for uranium with an enrichment of less than $20 \%$.

The facility in the conversion building mainly produces purified $\mathrm{UO}_{2}$ powder or $\mathrm{U}_{3} \mathrm{O}_{8}$ solution in nitric acid for the JOYO experimental fast reactor operated by Japan Nuclear Cycle Development Institute (JNC). Orders from JNC were few and irregular; thus JCO conducted nine campaigns in total (a campaign means a series of jobs for the production of JOYO fuel). For example, the ninth campaign was the first one in 3 years to make uranium solution. When a powder product $\left(\mathrm{UO}_{2}\right)$ is made, uranium material is put into the $\mathrm{UF}_{6}$ cylinder heater or the dissolution tank and the product is obtained from the blender. On the other hand, there are two processes for making a liquid product (uranium solution). In the first process - purification - handling of the uranium materials stops at calcination, and purified $\mathrm{U}_{3} \mathrm{O}_{8}$ is obtained. The purified uranium is dissolved in nitric acid in the second process - homogenisation. However, the method of homogenisation process has changed over 13 years (AIC document no. 7-2, 26 November 1999) after production of the uranium solution was begun (see section 3 ).

\subsection{Accident Summary Before 10:35, 30 September 1999}

The ninth campaign was aimed at producing a uranium solution by dissolving $57 \mathrm{~kg} \mathrm{U}$ with an enrichment of $18.8 \%$. The appointed date of delivery was in November 1999. The purification process started on 10 September 1999 and finished on 28 September, about 8 days earlier than the work plan. The preparation for the homogenisation process started on 28 September. Three workers were going to use the precipitation tank with a stir propeller instead of the buffer column. The tank is shared in the

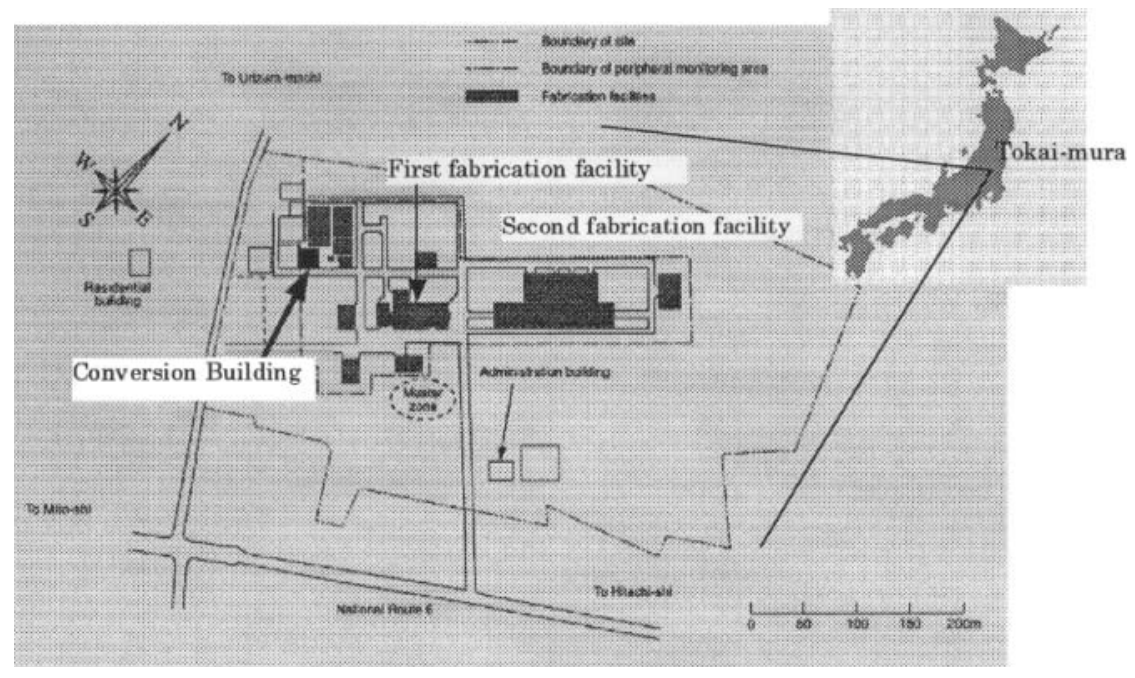

Fig. 1. JCO Tokai plant. 


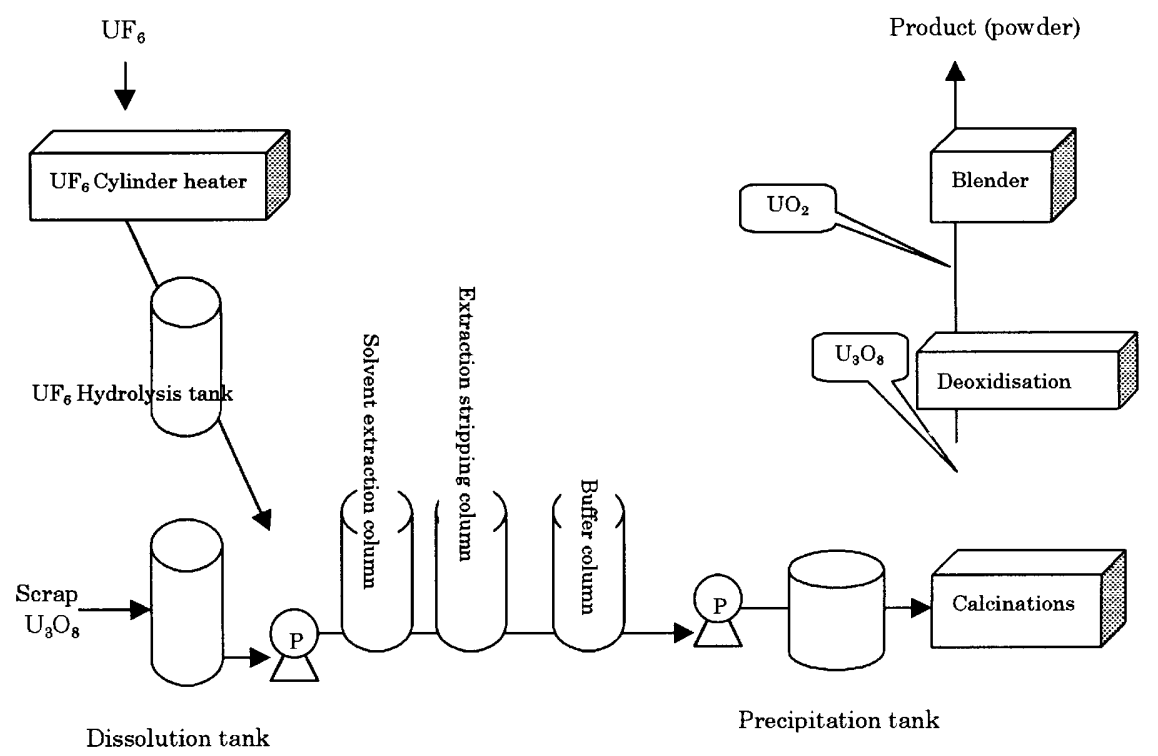

Fig. 2. Facilities in conversion building.

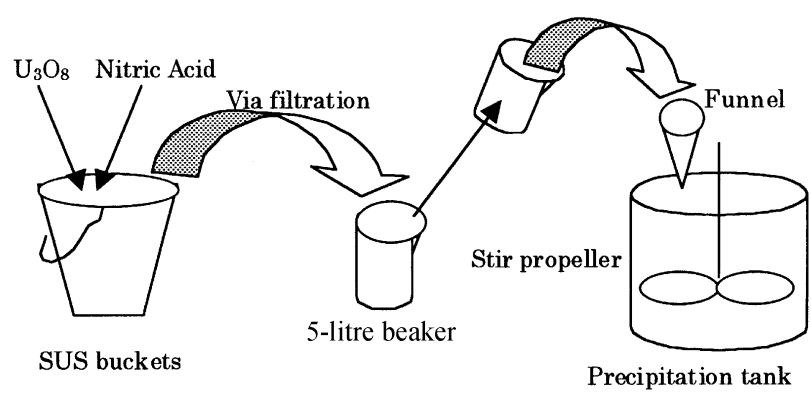

Fig. 3. Homogenisation in ninth campaign.

purification and homogenisation processes, so they washed the precipitation tank carefully, dissembling the lower part of the tank in order to maintain the quality of the product.

On the afternoon of 29 September, they started to dissolve $9.6 \mathrm{~kg}$ of uranium (four batches) with stainless steel (SUS) buckets and pour it into the precipitation tank through a small hole with a funnel (see Fig. 3). The hole was designed to enable the inside of the tank to be seen, not for pouring the solution. On the next morning, 30 September, they continued dissolving another $7.2 \mathrm{~kg}$ of uranium (three batches) in the buckets and poured it into the precipitation tank. At 10:35 JST, the first criticality accident occurred in the history of Japanese nuclear development. The total mass of uranium poured into the tank came to $16.6 \mathrm{~kg}$ out of $16.8 \mathrm{~kg}$.

\subsection{Accident Summary after 10:35, 30 September 1999}

This accident was first recognised when a warning went off, activated by a radiation monitoring system in the conversion building. At 10:55, the JCO Tokai plant set up its disaster prevention headquarters and it informed the head office in Tokyo of the accident at 11:00. The Science and Technology Agency (STA), Ibaraki Prefecture (local prefecture) and Tokai-mura (local municipality) received the first report from JCO, mentioning the possibility of a criticality accident at 11:19, 11:33 and 11:34 respectively. In the response to the report, the Agency notified the Prime Minister of the accident at 12:30. The Nuclear Safety Commission received the formal report of the accident at 14:00. On the other hand, Tokai-mura set up its disaster prevention headquarters at 12:15 and issued a sheltering indoors recommendation at 12:30. In response to JCO's request to evacuate residents within a $350 \mathrm{~m}$ radius, Tokai-mura issued a recommendation of evacuation to 150 residents within $350 \mathrm{~m}$ at 15:00. The final decision was made by the mayor of Tokai-mura. The government set up its headquarters with the Minister of STA as head at 15:00. Ibaraki Prefecture set up its headquarters at 16:00. The government upgraded the headquarters with the Prime Minister as head at 21:00. At 22:30, Ibaraki Prefecture issued a recommendation to shelter indoors to some 300,000 residents within a $10 \mathrm{~km}$ radius.

The cooling water around the precipitation tank was thought to be reflecting neutrons and enhancing the fission chain reaction. Therefore, at 2:30 on 1 October, an operation to drain the cooling water from around the precipitation tank was begun. Twelve pairs of JCO workers approached a pipe connected to the precipitation tank in order to drain the water and to pour boric acid into the precipitation tank. They were exposed to radiation and it took a few minutes to reach the exposure limit of $100 \mathrm{mSv}$. Finally, the end of criticality was confirmed at 8:30 on 1 
October. Later it was found that the criticality consisted of a very short period in the initial stage and the later stage in which the fission reaction continued slowly until 6:15.

As a result of this accident, the three workers performing the operation at the conversion building received some 17, 7 and $4 \mathrm{~Sv}$, respectively. The worker who received $17 \mathrm{~Sv}$ died after 83 days, and the worker with $7 \mathrm{~Sv}$ died 211 days after the accident. The other with $4 \mathrm{~Sv}$ went back to his job. The total number of people exposed to the radiation was 150 .

\section{CAUSE ANALYSIS}

\subsection{Changes of Procedure}

The immediate cause of this accident is clearly the pouring of some $16 \mathrm{~kg}$ of uranium into the precipitation tank - a procedure requiring mass and volume control. However, to understand the latent cause of this accident, it is necessary to review the 13-year period since JCO started the production of uranium solution. This chapter describes that period and then discusses the latent causes of this accident (Sasou et al 2000a, 2000b).

\subsubsection{Fourth Campaign: 1986-1988}

Production of uranium solution in nitric acid was requested by JNC. On the line of the licence conditions, JCO replied that they could make the solution with one batch of uranium. The volume of the solution would come to 6-7 litres. However, JNC further requested to increase the volume of the solution. JCO then had an idea to make 40 litres of uranium solution.

Uranium material was purified by passage through the dissolution tank, solvent extraction column, extraction stripping column, buffer column, precipitation and calcinations. In the homogenisation process, one batch of the purified uranium was dissolved in nitric acid in the dissolution tank. The solution was transferred to 5-litre stainless steel (SUS) bottles. This dissolution was repeated six or seven times until 10 SUS bottles of uranium solution were made. The solution was then homogenised by a method called 'cross-blending' (see Fig. 4). One tenth of

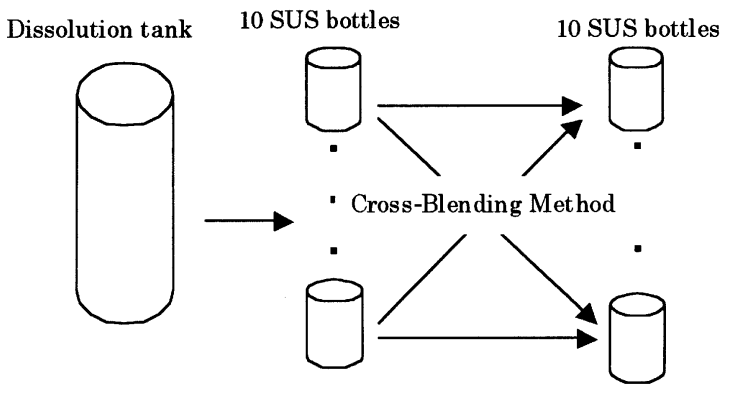

Fig. 4. Homogenisation in fourth campaign.

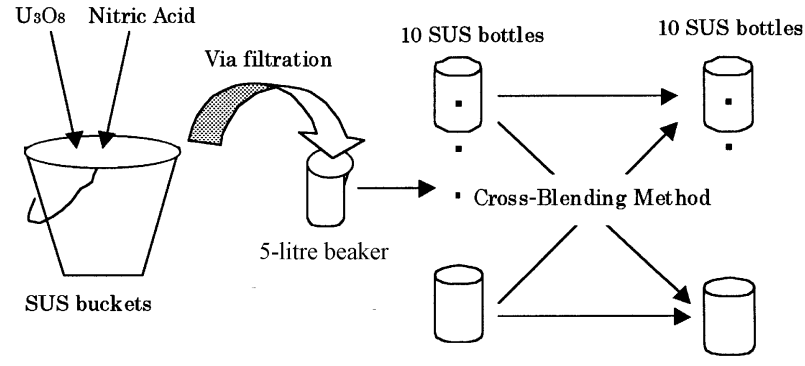

Fig. 5. Homogenisation in sixth campaign.

the uranium solution was taken from each bottle and put into another set of 10 bottles (AIC document no. 8-3, 4 December 1999).

Uranium solution was not produced in the fifth campaign.

\subsubsection{Sixth Campaign in 1993}

JCO hit a sales record of $¥ 3276$ million (\$32 million) in 1993 when JCO started to use SUS buckets. The dissolution tank was shared in the purification and homogenisation processes. Dissolution steps in both processes needed the dissolution tank at the same time. A worker or workers in charge of the work then hit upon using SUS buckets to dissolve uranium. This method was used in the homogenisation process. Every batch of purified $\mathrm{U} 3 \mathrm{O} 8$ was dissolved in a SUS bucket and six or seven batches of uranium solution were then homogenised by the crossblending method (see Fig. 5).

\subsubsection{Seventh and Eighth Campaigns: 1994-1996}

The method of using SUS buckets for dissolving the uranium was also applied to the dissolution step in the purification process in the seventh campaign starting in 1994. This was because the time taken to dissolve U3O8 material could be shortened from 30-90 minutes to 15-20 minutes per batch.

In September 1995 JCO's safety management committee discussed the deviations of the bucket-dissolving method from the government-authorised dissolution tank-dissolving method. They noted the illegality in using the buckets but recognised that it was criticality safe because the method considered mass and volume control in each bucket and the distances between those buckets. The committee then approved the bucket-dissolving method and made two minute notes. One was public and the other was confidential. The confidential one described what had been discussed in the committee. The public one, however, lacked this discussion (AIC document no. 7-2, 26 November 1999).

The homogenisation process in the seventh campaign started in October 1995. The purified uranium was dissolved with the SUS buckets and one of two buffer columns was used to homogenise the uranium solution (see 


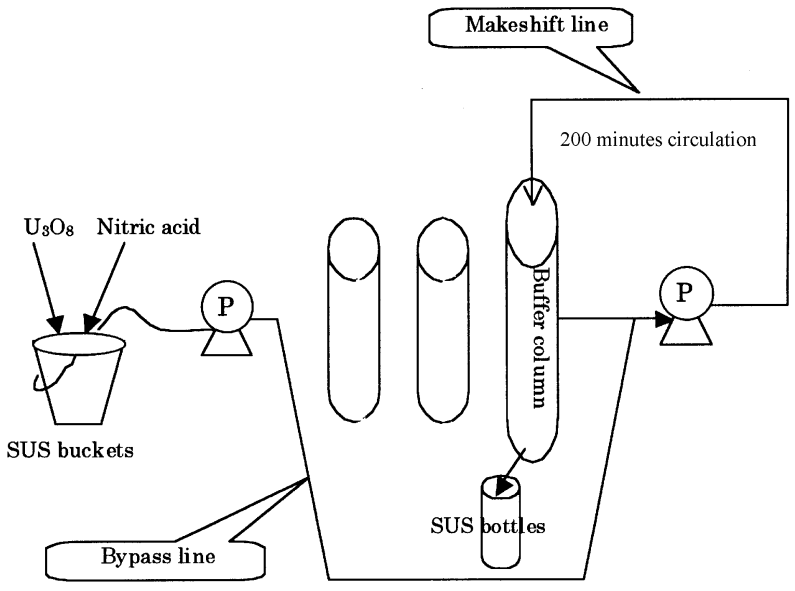

Fig. 6. Homogenisation in seventh and eighth campaigns.

Fig. 6). The buffer column and one of the pumps were separated from the process line and put into makeshift line to circulate the uranium solution in the buffer column with the pump for 200 minutes. The buffer column has a criticality safe geometry, so that six or seven batches of uranium solution could be put in it.

The same method was used also in the eighth campaign.

\subsubsection{Ninth Campaign in 1999}

In the original work plan, the purification process would start on 10 September and finish on 8 October. $54 \mathrm{~kg}$ of purified uranium would be divided into four lots of six or seven batches and the first homogenisation process would start on 29 September. Even this original schedule neglected the licence condition of batch control. According to the original plan, two or three batches could be between the dissolution tank and the precipitation tank.

The actual work further deviated from this illegal work plan. The purification process for all $54 \mathrm{~kg}$ of uranium was finished on 28 September, 8 days earlier than the original schedule (AIC document no. 4-6, 29 October 1999) and 1 day before the first planed homogenisation. The job record shows that there was a maximum of 10 batches of uranium between the dissolution tank and the precipitation tank. They seem to have hurried the purification process.

\subsection{Cause Analysis by J-HPES}

This section describes latent factors of the ninth campaign analysed by the J-HPES method (Takano et al 1994). Figure 7 shows the summary result of the J-HPES cause relation chart.

1. The workers were anxious to finish the job at the conversion building.

The three workers wanted some new workers who would be assigned to the special crew on 1 October to try the waste processing from the beginning. A group called special crew was in charge not only of operating the conversion building but also waste processing. In JCO, on-the-job training $(\mathrm{OJT})$ was the main training programme. It is surmised that waste processing would start in early October and the scheduled job for purification and homogenisation would interrupt the OJT on the waste processing for the newcomers. Therefore, they hurried the purification process and finished 8 work days earlier than scheduled.

The operation space was small and located far away, giving a feeling of entering the premises of another company. The conversion building locates the western end of the JCO site and is adjacent to a parent Sumitomo Metal Mining Co. site. The building, $15 \mathrm{~m} \times 16 \mathrm{~m}$ of a one-storey building, has a corridor leading to the Sumitomo building. The system designer never considered manual handling with buckets, so no space was reserved for manual handling. In addition, there were few jobs in the conversion building. It is possible that this work environment and rare opportunity made workers in the conversion building uncomfortable so that they hurried the job.

2. The workers decided to use the precipitation tank instead of the buffer column.

The system was designed for the purification process, so usability was poor and it took a long time to carry out the homogenisation process. The buffer column was shared in the purification and homogenisation processes so that workers had to cleanse the buffer column. However, it was of $17.5 \mathrm{~cm}$ diameter and $2.2 \mathrm{~m}$ height owing to the criticality safe geometry. It was quite hard to wash the interior. In addition, it was located only $10 \mathrm{~cm}$ above the floor so that it was difficult to take the solution from the bottom of the column. On the other hand, the precipitation tank was of $50 \mathrm{~cm}$ diameter, $70 \mathrm{~cm}$ depth and located about $1 \mathrm{~m}$ above the floor. The tank also had a stir propeller - useful for homogenising the solution. The three workers thought this very useful for the homogenisation process.

The precipitation tank has a capacity large enough to hold six or seven batches of uranium solution, because the precipitation tank was designed to handle uranium solution with an enrichment of $20 \%$ in $45 \mathrm{~g}$ U/litre. The whole system in the conversion building was designed for uranium with an enrichment of $12 \%$. However, in 1993, JCO installed another precipitation tank where the accident occurred in order to handle uranium with an enrichment of $20 \%$ under the approval of the authority. The capacity of the newly installed precipitation tank is 100 litres. On the other hand, six or seven batches of uranium solution come to 40 litres. The precipitation tank fitted their intention.

It was easier to pour uranium solution into a different tank because the three workers were using stainless steel buckets. Dissolving the purified $\mathrm{U}^{3} \mathrm{O}^{8}$ in stainless steel buckets was 


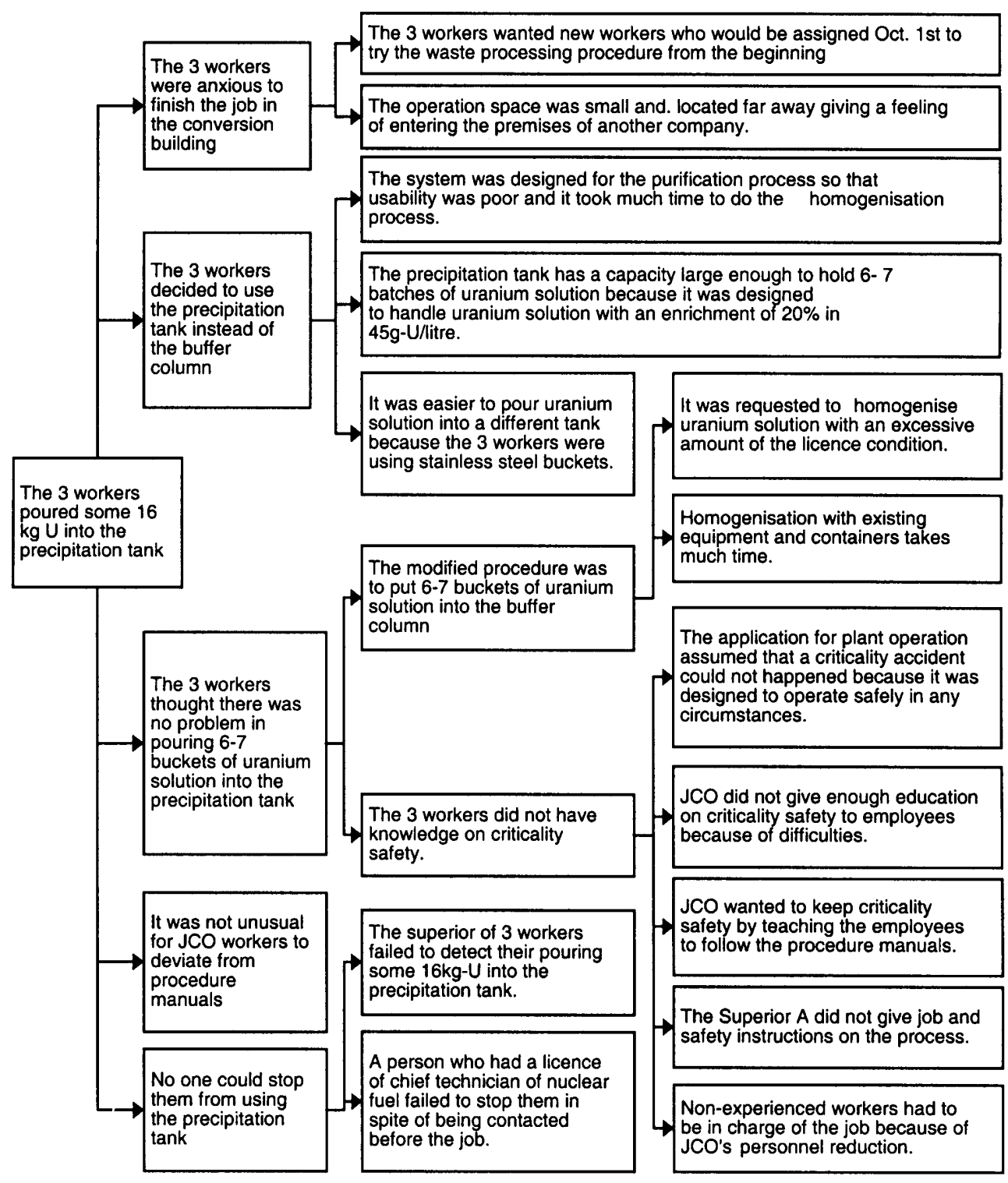

Fig. 7. Summary of J-HPES cause relation chart on pouring some $16 \mathrm{~kg} \mathrm{U}$ into precipitation column.

JCO's work habit since 1993. The workers could put the uranium solution dissolved in the buckets into any tank they wanted to use.

3. The workers thought that there were no problems in pouring six or seven buckets of uranium solution into the precipitation tank.

The modified procedure manual described how to put six or seven batches of uranium into the precipitation tank. This was the result of procedural changes over 13 years; and the manual only described how to make high-quality product, with no safety instructions.

The workers did not have knowledge of criticality safety. There were several latent factors behind this. First, the application for plant operation assumed that a criticality accident could not happen, because it was designed to operate safely in all circumstances. In the early days, JCO gave criticality safety education to its workers. However, it was difficult for the workers to understand it. Therefore, JCO stopped giving sufficient education on criticality safety and placed emphasis on teaching them to follow procedure manuals. Although placing emphasis on following the procedure manuals, the field supervisor in charge of giving instructions before the work did not give any job or safety instructions to the workers. In addition, because of the personnel reduction programme starting in 1996, JCO had no workers experienced in the job in conversion building, so that three workers without experience had to perform the job. 
4. It was not unusual for workers to deviate from procedure manuals.

Table 1 shows the relations between the processing method of the homogenisation process in the fourth, sixth, seventh and ninth campaigns, work period and the timing of editing the procedure manual. In all campaigns, there seem to have been no procedure manuals, and procedure manuals were edited after the jobs in the fourth and seventh campaigns. It seems that workers did not habitually follow procedure manuals in performing their jobs. Deviations from rules were also found in neglecting the work plans, described above. It seems that it is usual for workers not to follow rules such as procedure manuals, work plans, etc.

Table 1 Processing methods in the homogenisation process and manuals

\begin{tabular}{lll}
\hline Processing method & Work period & Manuals \\
\hline Dissolution tank and cross-blending method & $1986-88$ & 1989 \\
Buckets and cross-blending method & 1993 & Not available \\
Buckets and buffer column & $1995-96$ & 1997 \\
Buckets and precipitation tank & 1999 & Not available
\end{tabular}

5. No one could stop the workers from using the precipitation tank.

There were two people who could stop the three workers from using the precipitation tank. One was the supervisor of the workers. He was the man who did not give any job and safety instructions to the workers before the job. His other job was doing a round of inspection during work in the conversion building and checking work progress, etc. According to the investigation, he did the inspection at least once a day until 29 September. However, he failed to detect their pouring some $16 \mathrm{~kg}$ of uranium into the precipitation tank.

The other was a person who had made the job plan for the ninth campaign and had a licence of a chief technician of nuclear fuel. He was consulted by the workers' leader about using the precipitation tank during the lunch break on 29 September. He replied 'OK' in the early afternoon of that day. He did not stop them using the precipitation tank. He was quoted as saying that he had confused jobs in the first and second fabrication facilities and the conversion building.

\subsection{Cause Analysis by Extended CREAM}

Violation of rules has hardly been considered as a causal factor in conventional human reliability analysis. We tried, however, to extract causal factors of the accident using an extended version of CREAM (Cognitive Reliability and Error Analysis Method) (Hollnagel 1998), based on the assumption that changes in working conditions or the status of equipment can influence activities of a different organisation. The crucial erroneous action that directly caused the accident was that the workers poured some 16 $\mathrm{kg}$ of uranium into the precipitation tank instead of the buffer column. The error mode of this action is classified as 'wrong object'.

The focal points to be considered are threefold in the search for causal factors of this erroneous action. Firstly the performance of the three workers should be analysed, because they planned the action by themselves and executed it. Secondly the licensee of a chief technician of nuclear fuel consulted by the team leader should be included in the analysis. Thirdly the procedure manual should be considered, because the procedure of homogenisation with the buffer column violated the operational condition in the licence where both the mass and the batch control were requested. The following is a brief description of CREAM classification groups for the above points.

1. The workers made a plan to use the precipitation tank for homogenisation (inadequate plan).

One reason why the workers decided to use the precipitation tank rather than the buffer column was that they had to affiliate new members to the special crew on 1 October, and they wanted the newcomers to experience the waste processing from the beginning (inadequate task allocation). They therefore wanted to finish the work early in the conversion building (psychological stress).

Another reason was that the workers had difficulty in taking out the product from the buffer column (access problems), because the outlet of the buffer column was located just $10 \mathrm{~cm}$ above the floor (design failure). In addition, homogenisation with the buffer column takes 200 minutes, because the buffer column had been designed for the purification rather than the homogenisation process (design failure). Since they had experience of having used the precipitation tank in the purification process, they thought it would take less time to finish the job if they used the stirrer of the precipitation tank instead of the buffer column in the homogenisation process (hindsight bias).

The three workers had more than 10 years' experience in the first and second fabrication facilities, but the team leader had two or three months' experience, and the other two had just one or two weeks in the conversion building without any special education (insufficient knowledge).

2. The person consulted by the team leader made a wrong judgement that there would be no problems in changing the procedure (wrong reasoning).

While the field supervisor is authorised to change the procedure depending on the situation, the team leader consulted an unauthorised person, who is a licensee of the 
chief technician of nuclear fuel belonging to the planning group. The person consulted forgot that moderately enriched uranium was to be processed in the conversion building (memory failure) and that it would not become critical.

The person consulted was doing jobs usually on low enriched uranium in the first and second fabrication facilities but seldom on moderately enriched uranium. He misunderstood that mass control was not required with the buffer column, because the homogenisation process did not exist in the first and the second fabrication facilities. Since operation of the conversion building was infrequent and keeping specialists on this job was uneconomical, some standard for handling moderately enriched uranium should have been prepared, in particular for the homogenisation process (standard and rule problems).

3. When the procedure manual was revised to adopt homogenisation using the buffer column, they forgot to write clearly that the mass control was no longer valid (inadequate procedure).

Adoption of the homogenisation procedure using the buffer column was a kind of mechanisation, and it resulted in an improvement of safety compared with the cross-blending in the manual (perceptual bias). The technical managers, however, overlooked the risk that mass control was no longer valid with the buffer column in the homogenisation process (cognitive bias). The procedure manual describes a measure to prevent criticality in bottling the product, but it fails to clearly state that the buffer column is of a criticality safe geometry. When the manual was revised, it should have been stated clearly.

A draft of procedure manual is to be written by the production group, reviewed by the quality assurance group chief, and approved by the production group chief before validation. Revision of procedure manuals, however, requires no approval by the safety management group chief and the chief technician of nuclear fuel. This system was probably an organisational deficiency (inadequate task allocation).

Figure 8 shows a summary of the cause analysis by the extended CREAM. Although mechanisation of the homogenisation work by using the buffer column was an improvement of safety on one hand, the buffer column is no longer criticality safe by mass control on the other. The latter point was mentioned neither in the standard nor in the procedure manual for the homogenisation process. It was reasonable therefore for the workers to use the precipitation tank to finish the job early due to their insufficient knowledge and experience. The perceptual bias that made the organisation unaware of lack of mass control was crucial for the accident to occur.

\subsection{Summary}

It is a fact that the workers' unsafe act of pouring some 16 $\mathrm{kg}$ of uranium into the precipitation tank was the immediate cause of this accident. The analyses revealed many latent factors behind this unsafe act and most of them were found to be related to the company activities and work habits. This accident could be said to be an organisational accident as described by Reason (1997). Figure 9 helps us to understand this accident.

There were five defences designed to prevent criticality of the hazardous uranium. The first one was education. This first defence was destroyed by abandonment of giving education on criticality safety to employees by JCO. The second defence was procedure manuals and other kinds of documents. Deviations from the procedure were accepted by workers depending on the work situation. The procedure manuals and other kinds of documents described no safety instructions. Therefore, the second defence was breached easily. The third defence was the batch control required as a licence condition. However, homogenisation of six or seven batches of uranium broke this defence 13 years ago. The fourth defence was mass control. The effort to improve the quality and productivity by homogenisation with the buffer column destroyed this defence. The last defence, criticality safe geometry, was bypassed by using the precipitation tank. Finally, the criticality accident occurred in 1999.

We can find the root causes of this accident. The first is inappropriate decisions by plant management such as using a system designed for production of power products to produce liquid product, producing 40 litres of homogenised solution, the safety management committee's approval of the illegal method, etc. The company's decisions by executives or high-ranking managers gradually destroyed the defences. The second is poor management of process control, labour control, etc. Poor management gave workers opportunities to improve the processing methods. The third is poor corporate culture allowing neglect of rules. Even when someone neglected rules, there was no danger to the production and no punishment of the violator. On the contrary, illegal methods were approved by the company and became the official methods. Therefore, we can conclude that the poor corporate culture is the most important root cause of this accident and that it deflated the safety.

\section{PROBLEMS IN OPERATIONAL AND BUSINESS MANAGEMENT}

One of the popular explanations of the accident, which appears even in the official investigation report (AIC Final Report, 27 December 1999), is that the company pursued efficiency rather than safety because of a highly competitive 


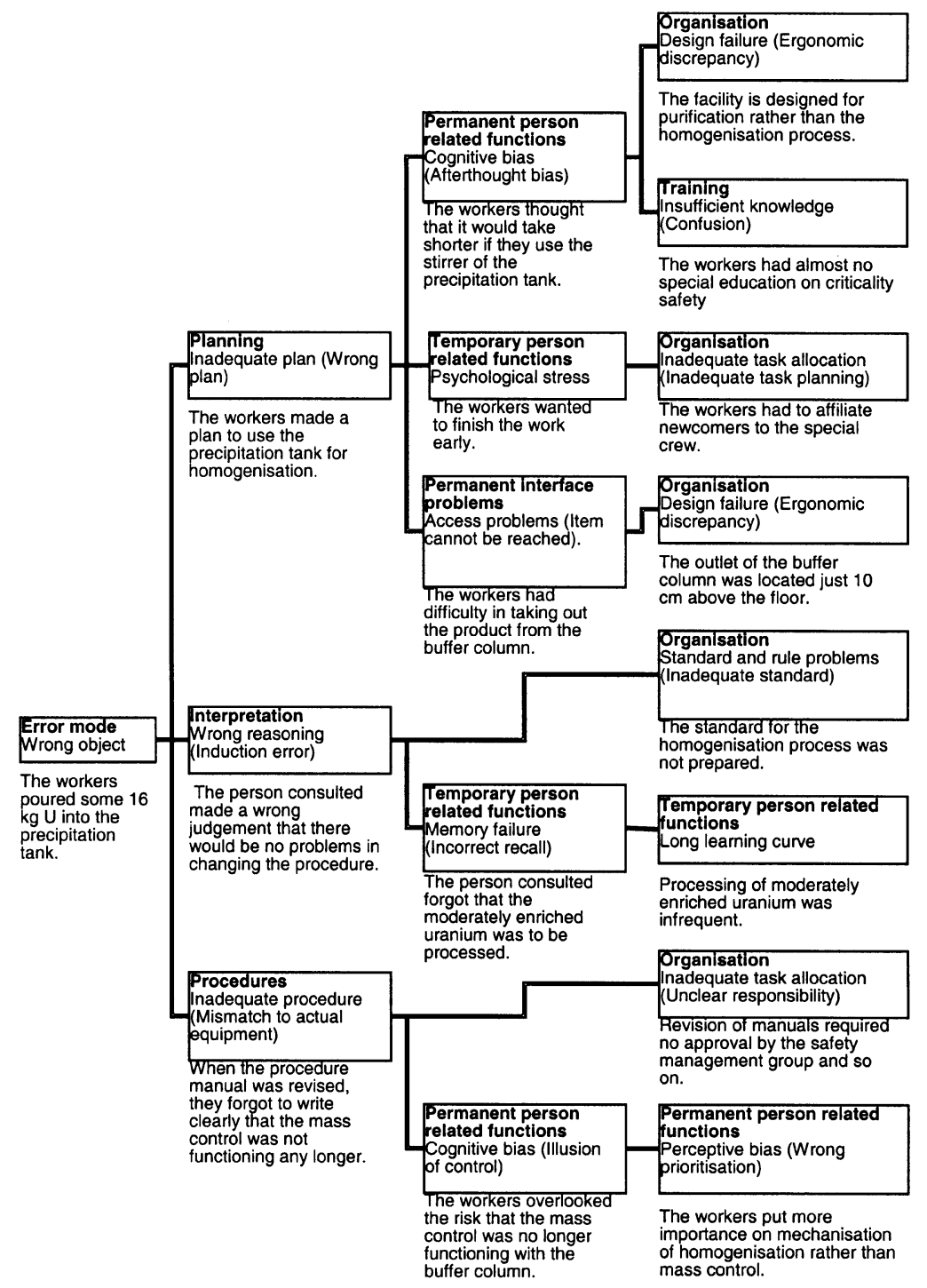

Fig. 8. Summary of cause analysis by extended CREAM.

climate in the international nuclear fuel business, that it reduced personnel, lowered ethical awareness in employees, and violated the authorised safety rules. A close look at the time-line of relevant events, however, reveals that the company started the violation while its business was growing. The above explanation therefore does not seem convincing. We have studied the operational management and business management of JCO to find out more plausible explanations.

\subsection{Organisation of Safety Management}

The organisation of safety management has been defined as shown in Fig. 10 by the business licence (AIC document no. 1-16, 8 October 1999) and the safety rules (AIC document no. 1-16-1, 8 October 1999) of JCO. The safety manager, who is concurrently the works manager, is responsible for safety management, and the chief technician of nuclear fuel and the safety management committee supports the safety manager. Practical jobs of safety management are to be shared by six administrators, but the safety administrator should play a more important role than the others.

Since the safety administrator is placed at the same level as the other administrators in the hierarchy, he can hardly take the initiative in the administration of field works unless the chief technician of nuclear fuel and the safety management committee have enough authority to assist the safety administrator. The safety rule has assigned nine plus four duties shown in Table 2 to the chief technician and the safety management committee respectively, but just three and two of these have actually been authorised in the company rules (AIC document no. 5-6, 5 November 1999). The two important positions for safety management 


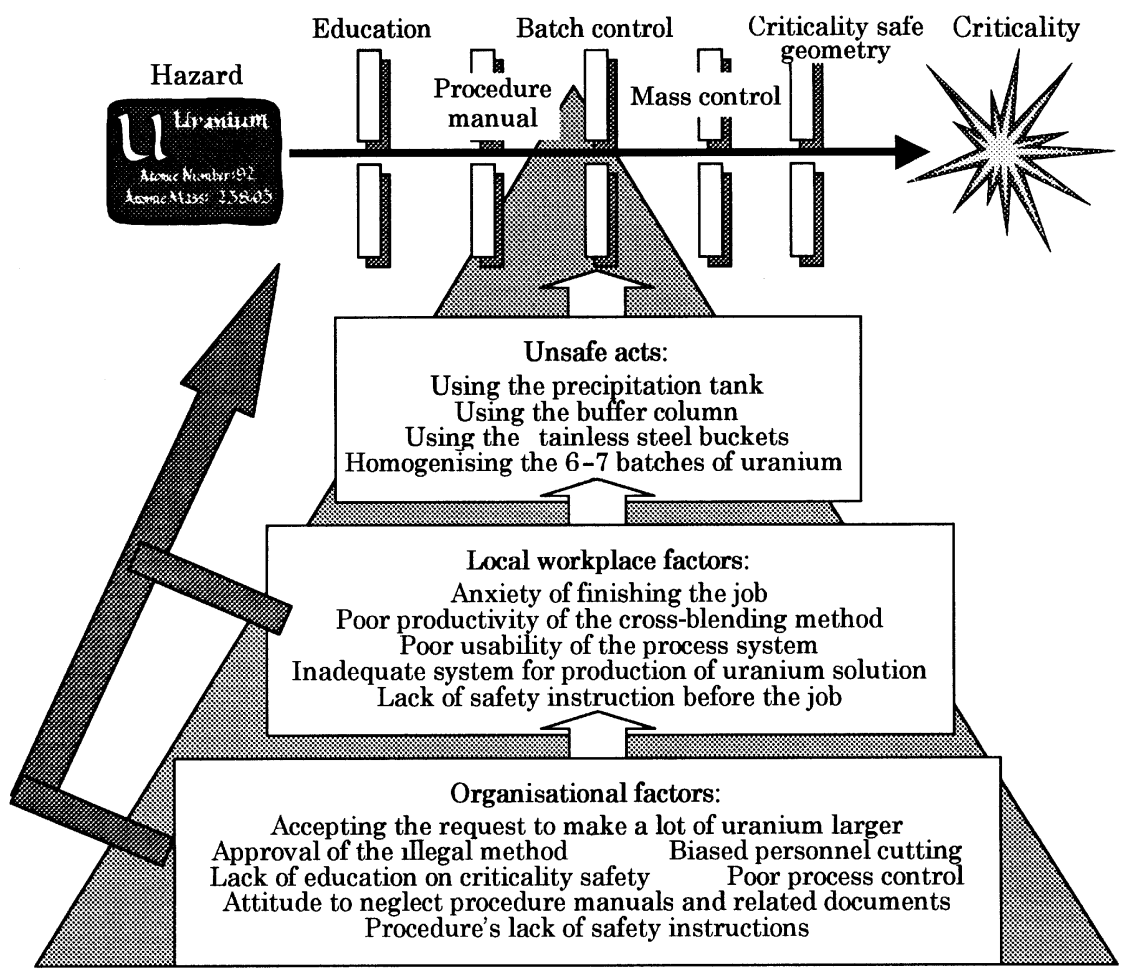

Fig. 9. JCO accident and organisational accident.

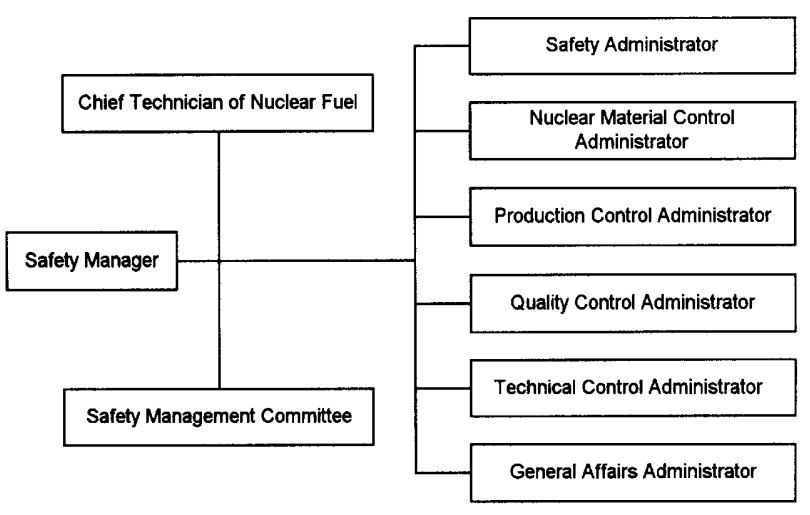

Fig. 10. Organisation of safety management defined by the business licence and the safety rule. therefore did not have enough authority to perform their duties.

Figure 11 shows the actual organisation in the company rule (AIC document no. 2-3-2, 15 October 1999), which almost complies with Fig. 10. A problem is that the same person in many cases is occupying two positions concurrently at the time of the accident, as shown in the figure. The works manager and the technical division manager, the production division manager and the production group chief, the planning group chief and the chief technician of nuclear fuel, the quality assurance group chief and the safety management group chief are combined. Although the organisation defined in the safety rules aims at efficient and reliable execution of safety management by specialisa-

Table 2 Duties of chief technician of nuclear fuel and safety management committee

\begin{tabular}{lll}
\hline & Chief technician of nuclear fuel & Safety management committee \\
\hline Safety rule & (1) Advice to the safety manager & (1) Issues of installation, reform and repair of primary facilities \\
& (2) Instruction to workers who handle nuclear materials & (2) Revision of the safety rule \\
(3) Advice and support to managers & (3) Participation in accident investigation, proposing remedies. \\
and their review & (4) Other issues relevant to safety management \\
& (5) Attendance to inspection by the regulatory body & \\
(6) Confirmation of reporting to the regulatory body & \\
(7) Confirmation of records as defined in the safety rule & \\
(8) Participation in education planning & \\
(9) Other issues relevant to safety management & (1) Licence application including safety assessment \\
(1) Licence application including safety assessment & (2) Revision of the safety rule \\
& (3) Education planning &
\end{tabular}




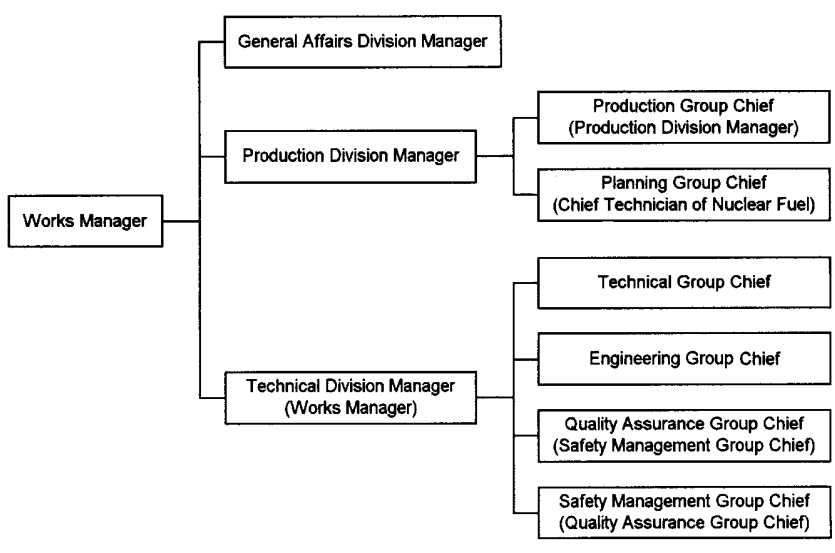

Fig. 11. Actual organisation of safety management at the time of the accident.

tion and cross-checking by different positions, it was probably inoperable owing to these multiple job combinations.

\subsection{System of Technical and Operational Management}

The company has administered and directed field workers with three types of documents: process parameter sheets, criticality control releases, and procedure manuals. Figure 12 shows the flow describing how these documents are to be issued and handed to field workers (AIC document nos 2-41 to $2-4-5,15$ October 1999). The three documents are issued based on a conversion process procedure, which is further derived from a quality assurance plan. A conversion process procedure and a quality assurance plan are to be drafted through consultation with the customer. Process parameter sheets and procedure manuals are to be based also on the criticality management rule, which is derived from the safety rules.

The safety management group does not participate in the preparation of the three documents for administration of field workers, nor do the chief technician of nuclear fuel or the safety management committee join this process. The three documents state mainly relevant points for quality assurance such as the amount of triuranium octoxide, nitric acid and water in the dissolution process. They lacked relevant points for safety management such as the criticality mass limit of uranium in a single batch, although it is stated in the criticality management rule (AIC document no. 5-6, 5 November 1999).

Judging from the above observations, the company administered and directed field workers mainly for the purpose of quality assurance rather than safety management. Reason (1997) pointed out that two kinds of manuals exist: manuals for production and those for safety. Manuals for quality assurance were the former, and no manuals for safety seemed to exist in JCO at least for the production process relating to the accident. This is in contrast to the eagerness of JCO for quality assurance.

Safety management and quality assurance are achieved partly by the same means, such as measurement, inspection, compliance with approved manuals and bookkeeping. This fact does not mean, however, that activities for safety management can be curtailed in substitution for activities for quality assurance, because the focal points for these purposes are different. It seems that the technical managers of JCO have misunderstood that.

\subsection{Activities for Business Improvement}

The operation procedures in the conversion building have been modified gradually since the first production of uranium solution at the facility in 1986: adoption of redissolution using SUS buckets, dissolution with SUS buckets in the purification process and homogenisation with the buffer column were the primary modifications (AIC document nos 2-3-3, 2-3-4, 15 October, and 9-5, 11 December 1999). Each modification has resulted in a great improvement in workability and operation time. These improvements were proposed and adopted by the production division without permission by the technical division and the safety management committee. The safety management committee discussed the discrepancy between the approved manuals and actual field works in 1995, and the committee admitted the changes and revised the manuals following the reality in 1996.

Such a pattern of revision where the technical division confirmed afterwards the change already adopted by the production division was repeated (see Table 1). It seems that the company has failed to establish a sound work custom to let the workers follow the approved manuals, because a cut-and-try manner of doing jobs has survived from the past developmental period of the facility. This time the workers hit on a procedure that deviated from the manual and adopted it after having consulted privately with a licensee of the chief technician of nuclear fuel who was not authorised to make the decision. The improper corporate culture described above must have allowed the workers to do so.

Activities for quality assurance have been encouraged and were in force before the accident (AIC document nos 2-4-2, 2-4-6, 15 October 1999). The field supervisor, who belongs to the production group, patrols the facility every day and checks operation records frequently. A patrol called QA (Quality Assurance) patrol has been enforced by the quality control group every 2 months, internal audits every year, and external audit by the proprietary company and the customers every few years. In the patrols, however, just the hardware of the facility and nuclear materials were inspected, and only documents and quality of products were of interest in the audits. These activities could not point 


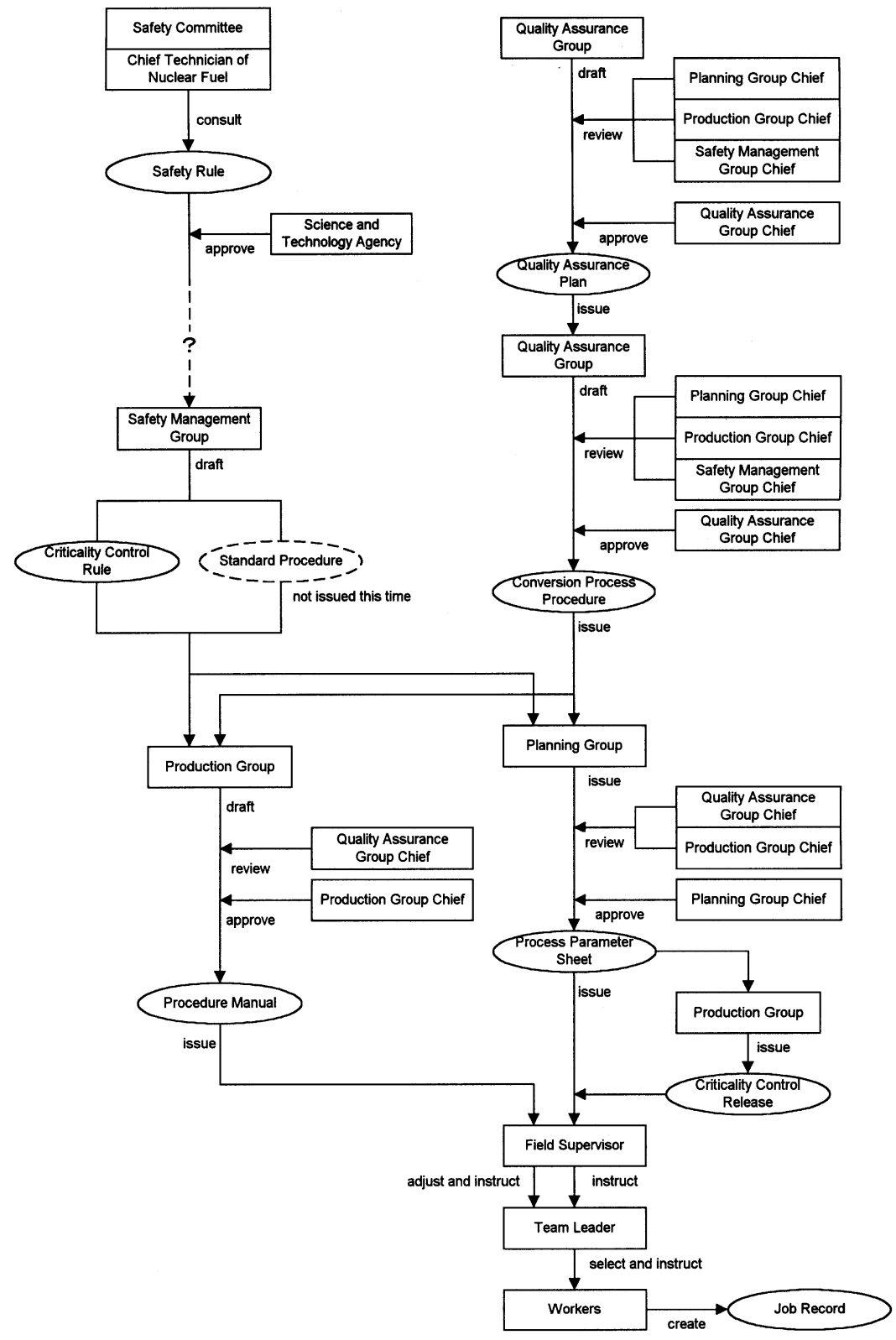

Fig. 12. Administration of field works.

out deficiencies in safety management and inconsistencies between the safety rules and the actual operation.

As safety promotion activities, the company encouraged employees to report small incidents and near misses, organised the safety and health committee to discuss relevant issues, and enforced safety patrols. As a result of these activities, the company has achieved a remarkable record of zero industrial casualties for the last 2 years. These activities have been dedicated, however, to avoidance of conventional industrial casualties, but the company overlooked serious defects in criticality safety. These activities should have been connected to avoidance of crucial accidents, but JCO has been interested exclusively in the quality of final products and avoidance of conventional industrial casualties. These cynical backgrounds should be better attended to prevent recurrence of organisational accidents of the same type.

\subsection{Risk Awareness and Education of Workers}

The JCO that could not prevent the workers from taking the unsafe action seems insensitive to latent risks in handling moderately enriched uranium. It may be attributed to the fact that radiation and nuclear reactions are invisible and unfamiliar in our everyday life. In a workshop where workers face such invisible risks, efforts of training 
workers to enhance sensitivity to the risks and visualising them should be continued. No particular considerations, however, were made in the manning, education or training system of JCO.

The workers learned the physics of criticality as well as criticality management principles in the introductory course for newcomers and annual safety education, but the course subject was superficial and the knowledge necessary for detecting danger of criticality was lacking. For example, topics such as what damage may result from a criticality accident, what is the minimum criticality mass, that moderately enriched uranium is more likely to become critical than slightly enriched uranium, and so on were not included in the material used in the tutorials (AIC document no. 4-6, 29 October 1999). The workers mentioned after the accident that they could not understand what they had been taught in the tutorials.

JCO put more stress on OJT than tutorial courses, but the company took no supplementary actions to ensure that workers learned knowledge that has little connection with field works. On the contrary, the approved procedure to feed uranium solution to the buffer column over the mass control limit is almost denying the criticality management principle taught in the courses. The company does not seem to have been concerned about important points of criticality management in the instruction of field workers. The company had no qualification systems for manning workers to the special crew who operated the conversion building.

The defects described so far suggest that both the technical managers and the executives were unaware of the latent risk associated with the nuclear fuel business, and that this unawareness resulted in overlooking the deviations from the safety rules and the approved manuals. A popular analysis claims that the unawareness has been caused by the company's indulgence in improvement of productivity after decline of the sales, but that is questionable. Figure 13 shows the trend of JCO's business and noticeable events before the accident. The sales hit a peak around 1993, and the crucial deviations from the safety rules began before this point, while the company was expanding its business. In the fourth campaign in 1986, for example, the limit on the amount of uranium to be put into the facility in one batch of the purification process was violated, and the cross-blending method, which was not approved by the licence, was adopted. In the sixth campaign in 1993, SUS buckets were used for redissolution of uranium. A lack of awareness of latent risks therefore seemed to exist before JCO experienced a difficult business situation. The root cause of the unawareness may be attributed to technical incomprehension of the top executives sent from the proprietary company as well as the assumption in the licensing that criticality accidents are unthinkable.

\subsection{Business Reform}

JCO conducted a large business reform, where site staff was reduced from 68 to 38, between 1995 and 1998 to reform the operational organisation owing to decline in sales. Meanwhile the number of teams operating the first and the second fabrication facilities has been halved from eight to four. As a result of this reform, production per worker in these facilities has increased from 10.3 to 18.7 tons of uranium. Although job demands for the special crew may also have increased, JCO claimed that the workload was not excessive from the records of overtime and holiday approval (AIC document no. 8-8, 4 December 1999).

The effects of the reform must be qualitative rather than quantitative. Two workers with enough experience in the conversion building had left the special crew after the last campaign in 1996, and 3 years had passed since then. All three workers who were engaged in the campaign this time had been working in the first and the second fabrication facilities for a long time, but they had almost no experience in the conversion building. Since mass control is not adopted in the first and the second fabrication facilities, they may have thought reasonably that they could put seven batches of uranium solution together without any problems.

As a result of the reform, the staff of the special crew have been increased, but they have been charged with various kinds of support jobs in addition to operation in the conversion building. The staff cut must have resulted also in the multiple job combinations already mentioned. When a person is charged with multiple tasks, he/she is likely to be interested more in the task for which the feedback to his/her efforts is clearer. The management, who decided the staff cut without any considerations of such human characteristics, probably overlooked erosion in safety defences due to degradation of job quality. This must be one of the primary causal factors of the accident.

\subsection{National Nuclear Development Plan and Nuclear Industry}

The main business of JCO is the manufacture of low enriched uranium fuel for light water reactors. Manufacture of nuclear fuel for the JOYO experimental fast breeder reactor (FBR) was small in quantity and infrequent. Despite its limited contribution of just $2 \%$ to sales, JCO must maintain the special facility and the special crew for this auxiliary business. It is questionable, therefore, whether a private company like JCO should participate in manufacture of such non-commercial products.

In the national plans for nuclear development launched in 1961, 1967 and 1972, the government announced the strong policy of domestication of nuclear fuel manufacture and commercialisation of uranium fuel manufacture. Following this policy, Sumitomo Metal Mining Co. Ltd 


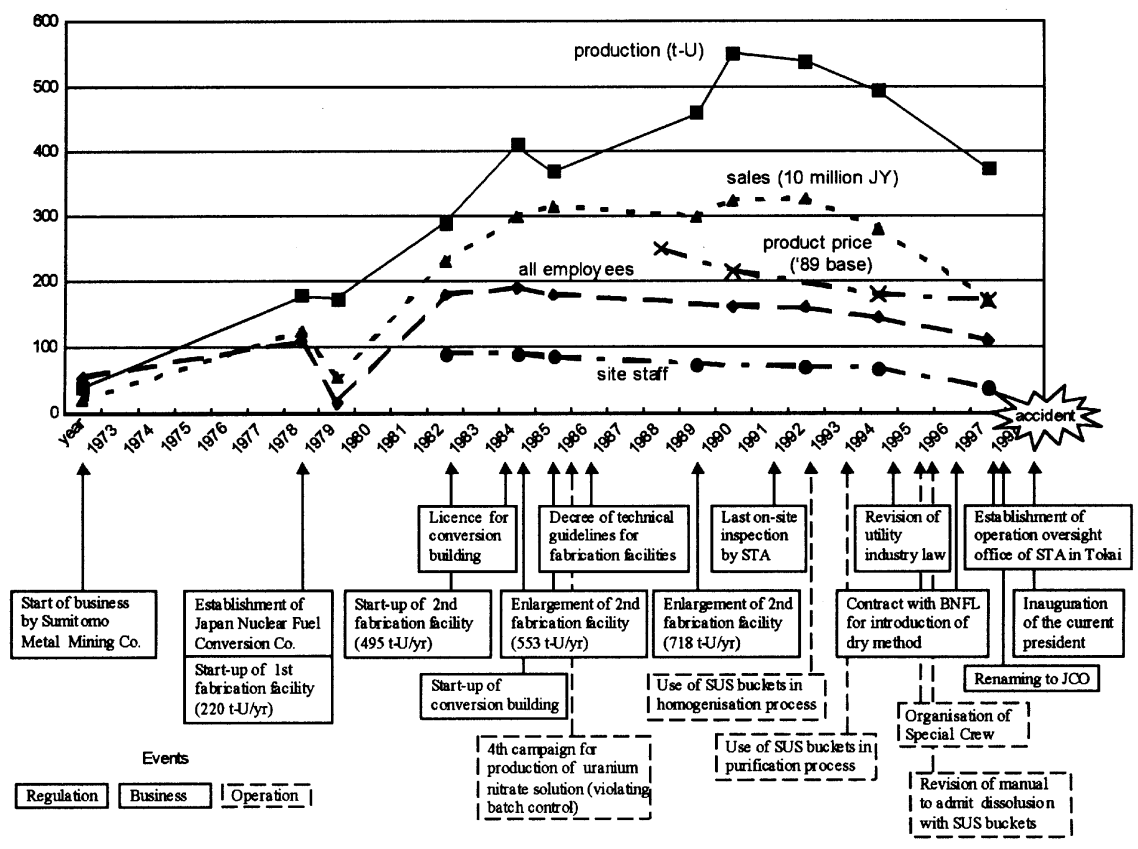

Fig. 13. Trend of JCO's business and noticeable events before the accident.

started research on methods of purification and conversion of uranium fuel, and began the business of uranium fuel manufacture including moderately enriched uranium for JOYO. The business was succeeded afterwards by JCO, Sumitomo's subsidiary company. JOYO is owned and operated by the Japan Nuclear Cycle Development Institute (JNC), and construction and operation of JOYO is a part of the national project of FBR development. Since introduction of the commercial FBR was expected within the twentieth century at the beginning of the project, both the government and Sumitomo Metal Mining must have expected that manufacture of FBR fuel would soon make a good business. Development of the FBR was delayed considerably though, and the expectation was not fulfilled. In this unfavourable situation, the government, JCO and its proprietary company should have reconsidered JCO's commitment to manufacture of the non-commercial product. Otherwise, the government should have provided the financial support necessary to maintain the national project.

The accident occurred during the process of mixing seven batches of uranium solution for homogenisation. This process seems contradictory to the criticality safety principle that production must be divided into small batches to prevent criticality. JNC requested JCO to homogenise the product (AIC document no. 8-3, 4 December 1999), because JNC could cut the labour and time of measurement of uranium density of the product, which measurement is required to get permission for transport of nuclear materials. Not only JCO but also JNC should have paid more attention to the side effects of the homogenisation process, but they consulted only within the negotiator level to decide. A background factor of this careless decision was that the cross-blending method for homogenisation, first proposed by JCO, is so popular in nuclear fuel processing that nobody noticed its latent problems in human factors. Another background factor may be the willingness of the contractor to meet any requests by the customer, which has been caused by a comparatively inferior position of fuel manufacturers in the nuclear industries.

\subsection{Remedies}

In summary, lack of collaboration between the production division at the front and the management at the back as well as insensitivity of the management to latent risks were the primary background factors that led to the accident. These unfavourable factors functioned interactively. The lack of collaboration allowed the front workers to deviate from the rules without permission, obstructed the management in checking the actual situation of field works, and allowed education and training of workers to rely heavily on OJT. Consequently the management became less sensitive to latent risks and more interested in productivity. At the same time the front workers became less sensitive also to latent risks due to inappropriate education and training. In addition to these factors within $\mathrm{JCO}$, the accident should be attributed also to inappropriate coordination between the national nuclear development plan and commercial business, and between the customer and the contractor. 
From the above analyses, we will propose the following remedies for accident prevention. The key points are how each enterprise can monitor the validity of its own safety management, and how the management can maintain their sensitivity to risks in the rear-supports to safety management. Firstly, sufficient authority and independence should be given to the positions relevant to safety management, and some concrete system to monitor the validity of inhouse activities should be implemented. In addition to selfmonitoring, audit by a third party is preferable. Secondly, viewpoints of safety management are to be integrated into quality assurance, and comprehensive activities for quality assurance are to be promoted. Thirdly, concrete actions must be taken to strengthen collaboration between the front-line workers and the rear-line management. Fourthly, education and training that can support workers to acquire elementary knowledge in conjunction with practical knowhow should be introduced. Instructions, devices and human interface that arouse workers' attention to latent risks are indispensable. Finally, a network of regulatory body, enterprises, and research institutes is to be established for exchange of safety-relevant information, experiences, lessons and ideas.

\section{ROOT CAUSE AS DEGRADATION OF SAFETY CULTURE AND SAFETY REGULATION}

The Special Task Force of AESJ has surveyed the causes of the JCO criticality accident based on the report of the Criticality Accident Investigation Committee for the Nuclear Safety Commission (AIC Interim and Final Reports etc., 1999), and has concluded that not only deviation from the government-authorised processing method, but also negligence of JCO's own illegal procedure manual resulted in the accident. The Committee commented that 'The primary responsibility of ensuring safety rests with business operators. The government plays a complementary role in helping business operators to ensure safety' in a section entitled 'Social Responsibility and Ethical Awareness of Business Operators and Engineers in the Nuclear Power Industry'. However, the root cause of the accident was considered a degradation of safety culture that occurred in the gap between commercial activities and safety regulation, while safety culture is a fundamental idea that supports all efforts to ensure safety in the nuclear industry. It is due to a lack of safety culture in Japan's nuclear community, not only within JCO and the regulatory bodies, but the lack extends also to many experts and engineers who could not predict that such a criticality accident could happen. With the criticality accident as a turning point, there is a greater call to bring the fundamental idea of safety culture home to the hearts and minds of the Japanese people. Based on this idea, efforts must be made to create a social system in which safety is given the highest priority.

\subsection{Problems in Licensing Procedure}

\subsubsection{Consideration of Safety Software}

The Committee pointed out 'A problem in the licensing procedure was that as the safety review, design and construction method review had focused on checking the appropriateness of a safety design for facilities and equipment and not on the detail of the operational procedure, adequate description on the redissolution process was not necessarily made in the safety review process.' This type of facility is considered as a category in which the highest level of safety must be established and great importance must be given to operational control. It is therefore urgently necessary to make a technical examination of the safety review and regulation, as well as the management system, operational procedures, and inspection and confirmation methods.

The Committee also commented that 'A problem found in business management was that the company did not pay full attention to the specific nature of the operation performed in the conversion building. This operation was smaller in scale and not frequently undertaken compared with the company's main operations in the first and second fabrication facilities. In order to prevent similar nuclear disasters, business operators should be required to take special safety control measures in the process of manufacturing special products in small quantities on an irregular basis. The fact that these processes have special characteristics must also be recognised.' This comment should be given also to the Nuclear Safety Commission and the regulatory bodies for reflecting to the safety review and inspection processes.

Considering that the operation in the conversion building of JCO was very particular, the facility should have been subjected to a review as a facility of special uses. In such a case, a safety review process, which will allow the regulatory bodies and the Nuclear Safety Commission to conduct double check-ups in a multiple, complementary fashion, is the most important.

The licensing process, including field inspection, should check the actual operation condition considering software aspects; that is, operation and control method of facilities, usability of apparatus, maintenance and management procedures, procedure manuals, operator level, education and training, etc. Experts of human factors should be included in this evaluation process. If this kind of licensing process had been performed, the problems - that the same facilities were used for processing both powder and liquid and that operability and usability were therefore terribly poor - would have been revealed in the safety review. 


\subsubsection{Consideration of Intentional Errors}

In any organisation, people tend to give priority on the logic of their inner circle, such as importance of economy or quality assurance of products. While safety operation is maintained, safety management activities or the importance of safety criteria are likely to degrade gradually. This kind of tendency is an open invitation to intentional errors such as violation of procedures by the organisation. New regulatory policies considering safety culture and safety evaluation processes are highly required for constructing a system to promote safety culture and to restrain intentional errors such as deviation from an authorised procedure, negligence of procedure manual, and so on.

When assessing the appropriateness of the basic design of facilities, equipment or components for criticality safety during a safety review, the following must be considered as preventive measures. Firstly, identify the conditions under which facilities, equipment or components are put to use. Secondly, consider the possibilities, if any, of deviating from these conditions when the facilities, equipment or components are put to use. Thirdly, implement specific safety designs of both hardware and software as well as the safety management of humanware, such as safety manual, education/training, etc., against not only wrongful operations but also intentional errors, while taking their potential dangers into consideration. Lastly, the impact of such deviations of concern must be studied as one of the maximum credible accidents. Returning to the original concepts of 'defence in depth', preventive measures for intentional errors such as 'fail-safe' and 'foolproof' should be installed in the facilities.

\subsubsection{Consideration of Potential Danger of Criticality}

The fundamental safety assessment guideline released in 1980 for nuclear fuel facilities requests that proper preventive measures for a criticality accident should be considered if there is any possibility of the accident due to operational errors. In contrast, the safety assessment guideline released in the same year for uranium-processing facilities, which handle uranium of an enrichment less than $5 \%$, states that consideration of a criticality accident is not necessary if the criticality safe conditions for single and plural components are satisfied. A problem is that the safety assessment guideline for uranium-processing facilities was restricted to enrichment of less than $5 \%$, and there were no guidelines for enrichment over $5 \%$.

\subsubsection{Retrofitting}

It is also important to point out that the government introduced a new regulatory guideline in 1980 for nuclear fuel facilities. The guideline says that a facility which handles uranium with an enrichment over 5\% should assume that the occurrence of a criticality accident is possible and take some measures to inform of the approach to criticality and to mitigate the consequences of accident. However, the management of the factory did not know the fact of introduction of the new guideline. Apparently, STA did not offer any guidance on voluntary retrofitting of the facilities to the new guideline. The Nuclear Safety Commission has not taken any action to call STA's attention to consider retrofitting to the new guideline and improvement.

\subsubsection{Licensee Upgrading Procedure}

JCO upgraded its enterprise in 1984 from a plant for treating only $12 \%$-enriched uranium powder to a processing plant licensed to treat powder and 20\%-enriched liquid. An in-depth evaluation was not made of the whole process, but only the modified parts, even though the same facilities were thereafter used for processing both powder and liquid and therefore operability and usability had become terribly poor. We should point out again that the licensing process, including the inspection procedure, should have checked the actual operation condition considering software aspects. Another problem is that the evaluation for a processing plant licensed to treat $20 \%$-enriched liquid was based on the fundamental safety assessment guideline for nuclear fuel facilities and the safety assessment guideline for uranium-processing facilities, which handle uranium of an enrichment less than $5 \%$.

\subsection{Problem in Safety Inspections}

\subsubsection{Periodic Inspection}

Processing plants such as JCO are exempt from required periodic inspections, unlike reprocessing plants or nuclear power plants, and consequently oversights of violations of procedures (organisational errors) can occur. The Accident Investigation Committee pointed out that 'A problem in the safety regulations was that the regulatory authorities failed to provide valid check-ups to ensure compliance with the safety rules.' In order to reinforce the inspection ability of the regulatory authorities, the following measures should be implemented: (1) conduct periodic inspections; (2) introduce a system of effectively checking compliance with the safety rules, (3) carry out inspections to check actual operation while the facilities are in service, and (4) carry out inspections without prior notice in an efficient manner.

\subsubsection{Consistency of Inspection Policy}

Currently, there are only 30 safety inspection members for over 200 facilities operated by various types of companies, while over 100 inspectors of MITI cover 51 nuclear power plants at 16 sites operated by only 10 large power companies. STA has failed to recognise or appeal to the government the fact that the number of inspectors is too small for the many facilities they should check.

Safety inspection members of STA inspected the JCO 
facilities twice recently, but as it was done during a period of non-operation oversight of such organisational errors occurred. Inspection systems to check safety activities of the facilities are insufficient. STA has paid little attention to the safety management of nuclear fuel facilities after the issuance of an operation permit, and made no efforts to employ the progress made in the administrative measures by MITI in the regulation of nuclear power plants. For example, there have been no resident inspectors, no periodic safety review, no systems for qualification of shift supervisors except chief technicians of nuclear fuel, no guidance for performing PRA studies and preparing management measures for a severe accident, no issues of back-fitting to the new guides, etc.

This deficiency might be brought about by the separation of nuclear regulation between STA and MITI, the former for non-power-producing reactors and non-reactor nuclear facilities, and the latter for nuclear power plants.

\subsection{Nuclear Safety Commission}

The locus of authority and the responsibilities among several entities in the field of nuclear energy were not necessarily clear. The underlying relations are between the Nuclear Safety Commission, regulatory administrative bodies and business operators. The Nuclear Safety Commission is required to exercise supervision and direction over safety administration independently from the regulatory bodies in order to meet the needs of society while taking a broad view of nuclear power plants and the nuclear fuel cycle. In order to fulfil its mission, the Nuclear Safety Commission should reinforce its secretariat in a drastic way and secure a group of technical advisers.

The Nuclear Safety Commission is a five-member team of part-time academic experts who have to check a huge number of documents produced by a small team of officials, who are far too few in number, and lack the expertise needed to regulate the safety of such a huge and potentially dangerous industry. Unless the government provides adequate independence, funding, manpower, expertise and accountability to a new regulatory body, the problems of the past will continue.

The government is encouraged to make further efforts to take the following basic measures in order to reconstruct the safety regulation framework:

1. Strengthen the independence of the Nuclear Safety Commission, reinforce its Secretariat, and secure a group of experts to assist it in various fields.

2. Reinforce staff of the safety regulatory authorities and clarify their role.

3. Formulate a complete set of examination guidelines and set up an efficient system of redundant complementary safety regulations.
Examining the safety-related organisations in the world suggests that a nuclear safety network consisting of a nuclear regulatory commission, a research institute of nuclear safety, and an advisory committee for nuclear safety is required.

\subsection{Actions by the Government}

With the criticality accident providing momentum, the Law for the Regulations for Nuclear Source Material, Nuclear Fuel Material and Reactors was revised to include numerous improvements, such as requiring nuclear fuelprocessing operators to conduct periodic inspections and introducing a system to check operators' compliance with safety regulations. It is a good recommendation that the Committee commented: 'The government should also pay greater attention to human factors and encourage operators to introduce the multiple protection, fail-safe concept in various aspects of nuclear operations.'

The government is required to assume full responsibility for developing a comprehensive design of the entire system, with the Nuclear Safety Commission taking a leading role. Furthermore, the government, local authorities and corporations should shoulder a proper share of the costs involved in materialising a safety-first social system. Cost for maintaining safety in the facilities should be recognised basically by business operators. However, if it is too expensive for a small-size private company, the government should support efforts to maintain its safety level, because the nuclear facilities are of social value. Especially if businesses are maintaining a long-range national project, necessary financial support should be provided, as mentioned in section 4.6. Meanwhile, efforts should be made to promote safety research projects to construct a safety infrastructure and push ahead with international education programmes for training engineering leaders with a strong sense of safety. In order to secure safety personnel, a constant effort to ensure safety must be seen as a goal that is as valuable as technological development.

\subsection{Chief Technician of Nuclear Fuel}

From analysis of the licensee examination tests for the past 10 years, chief technicians do not have sufficient knowledge of criticality safety, such as quantification of critical mass or instrumentation for criticality control, and therefore they do not have the necessary knowledge to predict danger of criticality accident. In addition to this, there is no periodic review of their ability after they have got the licensee. It is also a supplementary problem that the chief technician in JCO did not have proper authority or responsibility, mentioned in section 4.1. Safety regulation should include the sentence that the position of chief technician, such as his/her authority, responsibility, in- 
dependence, ability and so on, should be clearly stated in the safety rule of business operation.

\subsection{Risk Awareness}

Risk awareness means that one has a correct understanding of an event in which he/she or a third person is involved, foresees the effects of his/her actions, and recognises the potential risks behind those actions. Unless the awareness of risk is correct, there is no possibility of taking the proper measures or providing the support that will ensure safety before and after an incident. Therefore, risk awareness lies at the heart of the safety issue. In order to ensure safety, necessary preventive measures must be taken based on a full understanding of potential risks that may be in the background. An important measure that should be taken to attain risk awareness is to properly deploy key persons responsible for safety management and encourage employees to be always conscious of the risks involved in the tasks they undertake. A lack of awareness of the risk of criticality not only by JCO persons, such as field workers, technical managers, organisation of safety management, and executives but also by the regulatory bodies and the Nuclear Safety Commission was the underlying cause of the criticality accident. Correct risk awareness must be regarded as the starting point of all efforts to ensure safety. All organisations and individuals concerned with nuclear power must maintain risk awareness; that is safety culture.

In order to make risk awareness deeply embedded in society, we must change our attitude from the belief in the 'safety myth' and the notion of 'absolute safety' to the notion of 'risk-based assessment of safety'. It is now time to change the viewpoint from deterministic safety analysis to probabilistic risk assessment, and human reliability analysis is the key feature for the risk concept.

\subsection{Safety Regulation Considering Safety Culture}

An expert is one who knows problems in the fields and what safety is by long experience from responding to the problems that occurred in the early phase of technology improvement. In the mature technology, engineers or operators merely know safety by reading books, and lose the ability to reveal safety critical problems and to respond properly.

Various types of recent incidents/accidents have shown inherent organisational problems, and comparative analyses of incidents and evaluation of the common preventive measures for them are therefore required. An organisation will corrupt soon after it is created, that is, maintenance of the organisation itself will become self-purposive, while it had been originated to attain its inherent goal. In any organisation, people tend to give priority to the logic of their inner circle, such as importance of economy or quality assurance of products, rather than social prosperity or safety.

A respect of inner logic in the organisation is the opposite to the spirit of respect of safety culture. Risk awareness will degrade gradually due to respect of inner logic. A top-down approach is highly required for these inherent problems as organisational constitution. Respect of safety culture should be proposed and the process to evaluate it should be included in the safety regulations. The Nuclear Safety Commission has the duty of evaluation safety culture, and should consider the framework to support the business operator's own efforts to maintain safety culture. The way to construct business structure to maintain safety should also be included. A safety expert section, which has adequate independence, funding, manpower, expertise and accountability, should be established as an obligation in the organisation.

\subsection{Duty of Academic Society}

Academic society should discuss the following issues and propose effective countermeasures to the regulatory bodies, nuclear engineers and also to the public:

1. What is safety? How safe is safe enough?

2. Methodology to prevent the degradation of safety culture.

3. Methodology for technology inheritance (fields, safety).

4. Methodology of PSA and HRA and its reflection.

5. Methodology of root-cause analysis and its reflection.

6. Manual/document writing technique (know-why).

\subsection{Summary}

The primary cause of the accident was clearly lack of attention to criticality safety due to inadequate education and lack of spirits of observing the technical specifications in the plant. The root cause was inattention to the licensee's safety management at non-reactor nuclear facilities by the regulatory body after issuance of the permission of operation.

Even in fields with mature technologies, accidents exceeding the limits of safety engineering designs have recently occurred frequently. The criticality accident suggests that as the development and improvement of hardware types of safety precautions have reached a saturation point, the creation and materialisation of software types of safety securing measures and safety support measures are gaining importance. Various types of recent incidents/accidents have shown inherent problems concerned with risk awareness, and comparative analyses of incidents and evaluation of common preventive measures for them are therefore required. 


\section{PROBLEMS IN ADMINISTRATIVE RESPONSE}

At 15:30 on the day of the accident, the government of Tokai-mura recommended evacuation within a $350 \mathrm{~m}$ radius. At 22:30, Ibaraki prefecture requested people within a $10 \mathrm{~km}$ radius to shelter indoors. It was the first nuclear accident in Japanese history and forced people to evacuate or to shelter indoors. In this chapter, we will describe some problems of administrative emergency responses in the accident, such as information gathering, decision making and public information.

\subsection{Administrative Countermeasures for Nuclear Disaster Before the Accident}

In Japan, under 'The Disaster Countermeasures Basic Act', national and local governments are responsible for protecting people's lives and property against disasters. The national government has prepared 'The Basic Plan for Disaster Prevention' as the basis of the nation's disaster prevention measures. This plan contains various countermeasures for each type of accident and disaster (earthquakes, volcanic disasters, floods, traffic accidents, etc.) Each local government should also prepare 'The Local Plan for Disaster Prevention' after this national plan.

The Basic Plan for Disaster Prevention was drastically revised in June 1997 after two nuclear incidents: a sodium leak at the demonstration fast breeder reactor MONJU (December 1995) and fire and explosion at the low-level waste-processing plant at Tokai-mura (March 1997). In the new plan, countermeasures for nuclear disasters became much more concrete and practical. Each local government started to revise their local plans too; Ibaraki prefecture and Tokai-mura had one of the most advanced local plans for nuclear disasters, especially with regard to public information. They had prepared a plan for emergency announcement and delivered wire-less receivers to each household.

In the Disaster Countermeasures Basic Act, local governments are responsible for declaring evacuation and/ or sheltering indoors to residents around nuclear plants, but it was said to be very difficult to make such a decision accurately, for the following reasons:

1. There are only a few experts on radiology or nuclear engineering in local governments.

2. Local governments have only limited means for information gathering compared with STA or MITI, who regulate nuclear industries.

3. The influence of nuclear disaster would possibly extend beyond one administrative district, so it would not be possible for local governments to make those decisions only by themselves.

\subsection{Problems in Emergency Responses}

During the accident, there were many problems in emergency responses of each organisation. What we should learn from the accident for the future is as follows:

\subsubsection{Emergency Reporting from JCO}

The emergency reporting from JCO plant was neither accurate nor quick enough, which caused two problems.

The first problem was some delay in administrative emergency responses. The Tokai-mura office received the first report on the accident right after it occurred, because the village had made an agreement on early reporting with JCO. Based on the first report, the village office started an emergency response. But in Naka-machi, neighbouring Tokai-mura and very close to the JCO plant, the local government did not realise that the accident had occurred until about 2 hours later, when people started sheltering indoors in Tokai-mura. Other cities and towns did not receive any emergency reports from JCO either, as they had no agreements or preparedness plans for emergency reporting. As a result, all cities, towns and villages within a $10 \mathrm{~km}$ radius except Tokai-mura were behind in first response.

The second and more critical problem of emergency reporting from JCO is that at first it did not include any information about the possibility of a nuclear accident. At 10:43, JCO called for an ambulance from the Tokai fire department, but did not clearly mention that they had an accident in their facility. As a result, the ambulance crew, who did not prepare for radiation protection, were exposed to radiation at some level.

\subsubsection{Decision Making and Execution of Evacuation in Tokai-mura}

The Tokai-mura office set up its disaster prevention headquarters at 12:15 and gave residents a warning to refrain from going outdoors three times, at 12:30, 12:55 and 13:30. After 14:00, a person from JCO visited the headquarters and requested evacuation within a $500 \mathrm{~m}$ radius around the plant. The headquarters, with some advice by experts from the Japan Atomic Energy Research Institute (JAERI) Naka branch, decided to recommend evacuation within a $350 \mathrm{~m}$ radius. The reason of this reduction from $500 \mathrm{~m}$ to $350 \mathrm{~m}$ was that a $500 \mathrm{~m}$ radius extends beyond the village boundary to Naka-machi, where the local government had not yet set up its headquarters.

Another important point is that the headquarters did not declare but only gave a recommendation for evacuation. As mentioned above, the local government could declare evacuation and/or sheltering indoors under the act. But actually it was difficult for a small government like Tokaimura to make this decision, which might have serious impacts on the local society and economy, without any approval from national agencies. At the time the village 
headquarters was discussing an evacuation, no national authorities nor Ibaraki prefecture had set up their headquarters, and it was impossible for the village government to receive any advice or approval from them. Therefore, the headquarters of Tokai-mura could only recommend evacuation, which has less sense of enforcement.

The headquarters of Tokai-mura first informed community leaders about the evacuation recommendation, then visited each household to make people assemble at the refuge. For their transport, some nuclear-related organisations in the village were asked to lend their buses, and the Tokai-mura government dispatched almost all of its own cars also. It is said that the number of cars and buses was as large as the number of evacuating families. There were some residents who hesitated in leaving their houses. Village officers patiently persuaded them to evacuate and go to the refuge.

In this case, the national government could hardly take an initiative in the emergency response, but the Tokaimura government was able to make the decision accurately, because the village mayor had a good sense of risk management against nuclear disaster. Moreover, there were many research institutes of nuclear engineering in the area, which enabled the mayor to receive advice from experts. We should emphasise the fact that it was because of Tokai-mura that the local government could make the decision. No other local government would be able to make such a decision without the national government's advice and approval.

\subsubsection{Decision Making and Public Information on Sheltering Indoors}

In the evening, Ibaraki prefecture government started to discuss the request of sheltering indoors. The government set the boundary of sheltering indoors within a $10 \mathrm{~km}$ radius, according to its prescribed plan, which says the sheltering area might be within an $8-10 \mathrm{~km}$ radius. The Ibaraki headquarters asked for some technical advice to the national government on the boundary setting, but the national government only approved the idea and did not provide any technical or scientific background.

Even after the headquarters decided to request sheltering indoors, it took much time for the headquarters to announce this officially. The reason for this delay has not been identified, but it caused a kind of disorder as a TV news broadcast reported the sheltering warning before the headquarters made it public. As a result, there were floods of inquiry telephone calls from residents to the local governments, who were not officially informed about the warning in advance.

\subsubsection{Actions to Terminate Criticality}

It took a long time for experts to realise that a nuclear chain reaction was still continuing, and actions to terminate criticality were delayed. They were forced to make judgement depending on uncertain information, because criticality accidents had not been assumed in licensing and the plant was not equipped with neutron detectors. Many experts supposed that a chain reaction would have terminated due to scattering of the uranium solution by the burst. It was around 17:00 therefore when the continuation of criticality was confirmed by measuring the neutron dose. JAERI Naka branch noticed some anomaly in the indication of their neutron monitor, measured the neutron dose voluntarily, and sent the data to STA. The data, however, disappeared under a pile of many fax papers and did not reach the experts in time. Since criticality accidents had not been assumed, the plant was not equipped with devices to terminate criticality either. Preparation for the actions relied on other nuclearrelated organisations such as JAERI; it took a while to plan concrete means to terminate criticality, to prepare borated water, and so on. The actions were administered by the local headquarters of the Nuclear Safety Commission, but the locus of legal authority for ordering works with exposure to radiation was unclear.

\subsubsection{Cancelling the Request and the Recommendation} The request of sheltering indoors had been cancelled in the afternoon of the next day. But the timing of publication differed for each headquarters, which indicated confusion regarding who should take the initiative in cancellation.

As for the evacuation recommendation, the evacuated people were of course eager to go home as soon as possible, but not before safety was confirmed. They needed to be informed why they should stay in the refuge, and when they would be able to go home. In the afternoon of 2 October, the mayor of Tokai-mura visited the national headquarters, where many experts were executing countermeasures against radioactivity, but received no other information other than 'now under consideration'. The mayor had much difficulty in explaining the situation to the people in the refuge, and he was even prepared to kneel down on the ground to ask people to stay in the refuge.

As is the case in any disaster, cancelling warnings is a very difficult matter in a nuclear disaster. It should be clearly defined who is going to make the decision on cancelling, and how authorities and organisations related to the decision should coordinate. It is also important to disclose all information during discussions so that people can understand the meaning and reliability of the warning.

\subsection{For Better Administrative Nuclear Emergency Responses}

After the accident, a new law named 'The Special Measures Act for Nuclear Disaster Countermeasures' was enacted. Under the new act, the national government is responsible for all nuclear disaster countermeasures including emer- 
gency response such as declaring evacuation and sheltering. The concrete countermeasures are still under planning, but we have the following proposals to put this act to practical use.

\subsubsection{Establishing Practical Decision Criteria for Nucle- ar Emergency}

Under the new act, the national government will declare an emergency when needed. This would enable a more effective response for both national and local governments. On the other hand, it might cause some delay to those responses, as the national response did in the JCO accident. The early and appropriate decision that was made by the mayor of Tokai-mura will not be possible under this act.

The national government is establishing numerical decision criteria on nuclear emergencies, although it is not enough. There might be some incidents that cause little radiation leakage but much anxiety and fear in residents. To prepare for such unforeseen circumstances, it is necessary to establish the criteria considering people's psychological aspects.

\subsubsection{Coordination Between National and Local Govern- ments in the Off-Site Centre}

Under the new act, the national government should set up its headquarters on the spot in a nuclear emergency, which is called the 'off-site centre'. Members from national and local authorities and other related organisations such as police, self-defence, emergency medical services, telecommunication companies, news media, etc. will assemble in the centre.

It is useful that all related organisations come together and exchange information to coordinate their emergency responses. Especially when the national government issue evacuation or sheltering counsel, basic information on the community and residents is essential to avoid any confusion or disorder, and the local government should play an important role in providing such information.

We hope that national and local governments and other organisations establish a preparedness plan, in which each role is clearly and practically defined, so that they can work in an effective coordinated way during an emergency.

\subsubsection{Effective Drills and Exercise for Nuclear Disaster} It is also stipulated by the law that each organisation related should carry out a 'comprehensive drill for nuclear disaster response' every year. There also were some drills executed in the past which, however, were not practically significant but rather a kind of 'show' in which every person only followed a prescribed scenario.

After the experience of the JCO accident, it is important to have drills and exercises where every participant is forced to think and make decisions on his/ her own. Through these drills we should confirm and improve our emergency response plans for future accidents.

\section{CONCLUDING REMARKS}

As described so far, inappropriate ergonomic designs of the facility and the procedure as well as deficiencies in safety management by the company facilitated the workers' unsafe action that caused the accident. Behind these direct causes, several organisational problems have been found in the business culture of the company and the safety administration by the regulatory body. The workers' unsafe action therefore is not a conventional human error, but it should be categorised as an organisational error. Inappropriate response to the accident, which enlarged the impact of the accident, was also caused by organisational errors such as inappropriate communication and inappropriate role assignment between the company, the central government and the local governments. Instant patches of direct causes such as remedies of equipment, intense training and control of workers, and enforced oversight and inspection cannot prevent organisational accidents caused by such organisational errors. Remedies and countermeasures are required considering organisational factors in the background.

We will not repeat here the particular causes of the accident and the corresponding remedies, since they have been discussed in the previous sections. The primary cause of the accident was, in summary, that safety-relevant information such as principles, knowledge and lessons were not shared among different organisations related to the nuclear industry in Japan. Close exchange of safetyrelevant information seemed lacking between the production division and the management division within JCO, between different sections in the regulatory body, between the regulation officials at the time of licensing and their successors, between the central government and the local governments, and between different sectors in the industry. Consequently, efforts to establish safety culture over the nuclear industry as a whole were insufficient, and an unfilled zone of safety culture had not been detected.

As a lesson learned from the accident, we should keep in mind that safety culture can hardly be established just within a particular organisation isolated from others. Safety culture can be established and maintained by exchanging safety-relevant information between different organisations, industry sectors and times, by arousing risk awareness, and by learning from experience together. We hope the Nuclear Safety Network established after the accident by private companies and research institutes related to the nuclear industry in Japan might play the key role. In addition, academic communities, which have been apt to move separately within specialised narrow areas, should discuss safety principles and generic ideas of safety management over different research areas and lead society towards a good safety culture. 


\section{Acknowledgement}

The authors express their thanks to the participants of the Special Task Force for JCO Criticality Accident Investigation, the Division of Human-Machine Systems Studies, and the Atomic Energy Society of Japan. We also appreciate the assistance of those who provided confidential information or joined discussions on the accident.

\section{References}

Hollnagel E (1998). Cognitive reliability and error analysis method (CREAM). Elsevier, Oxford, UK.
Reason J (1997). Managing the risks of organizational accidents. Ashgate, Aldershot, UK.

Sasou K, Goda H, Hirotsu Y (2000a). An analysis on human factor issues in criticality accident at a uranium processing plant. CRIEPI report no. S99001 (in Japanese).

Sasou K, Goda H, Hirotsu Y (2000b). Human factors analysis on criticality accident. In 5th nuclear energy symposium, Beijing, China, 27-29 March.

Takano K et al (1994). System for analyzing and evaluating human-related nuclear power plant incidents. Journal of Nuclear Science and Technology 31(9):894-913.

Correspondence and offprint requests to: K. Furuta, Institute of Environmental Studies, University of Tokyo, 7-3-1 Hongo, Bunkyo-ku, Tokyo 113-0033, Japan. Email: furuta@k.u-tokyo.ac.jp 
APPENDIX D 


\title{
Qualitative Human Reliability Analysis for Spent Fuel Handling
}

By

\author{
Jeffrey D. Brewer*, Paul Amico ${ }^{\dagger}, \&$ Susan E. Cooper ${ }^{*}$ \\ * Sandia National Laboratories, P.O. Box 5800, Albuquerque, NM 87185-0748, jdbrewe@sandia.gov \\ ${ }^{\dagger}$ Science Applications International Corporation \\ * United Stated Nuclear Regulatory Commission
}

Human reliability analysis (HRA) methods have been developed primarily to provide information for use in probabilistic risk assessments (PRAs) that analyze nuclear power plant (NPP) operations. Given the original emphasis of these methods, it is understandable that many HRAs have not ventured far from NPP control room applications. Despite this historical focus on the control room, there has been growing interest and discussion regarding the application of HRA methods to other NPP activities such as spent fuel handling (SFH) or operations in different types of facilities. One recently developed HRA method, 'A Technique for Human Event Analysis' (ATHEANA) has been proposed as a promising candidate for diverse applications due to its particular approach for systematically uncovering the dynamic, contextual conditions influencing human performance. This paper describes one successful test of this proposition by presenting portions of a recently completed project in which a scoping study was performed to accomplish the following goals: (1) investigate what should be included in a qualitative HRA for spent fuel and cask handling operations; and (2) demonstrate that the ATHEANA HRA technique can be usefully applied to these operations.

The preliminary, scoping qualitative HRA examined, in a generic manner, how human performance of SFH and dry cask storage operations (DCSOS) can plausibly lead to radiological consequences that impact the public and the environment.

The study involved the performance of typical, qualitative HRA tasks such as collecting relevant information and the preliminary identification of human failure events or unsafe actions, relevant influences (e.g., performance shaping factors, other contextual factors), event scenario development and categorization of human failure event (HFE) scenario groupings. Information from relevant literature sources was augmented with subject matter expert interviews and analysis of an edited video of selected operations. Elements of NUREG-1792, Good Practices for Implementing Human Reliability Analyses

(HRA) and NUREG-1624, Rev. 1, Technical Basis and Implementation Guidelines for A Technique for Human Event Analysis (ATHEANA) formed critical parts of the technical basis for the preliminary analysis. Misloading of spent fuel into a cask and dropping of a loaded cask were the two human failure event groupings of primary interest, although all human performance aspects of DCSOs were considered to some extent.

Of important note is that HRA is typically performed in the context of a plant-specific PRA study. This analysis was performed without the benefit of the context provided by a larger PRA study, nor was it plant specific, and so it investigated only generic HRA issues relevant to SFH. However, the improved understanding of human performance issues provided by the study will likely enhance the ability to carry out a detailed qualitative HRA for a specificNPP at some point in the future.

Furthermore, support was obtained regarding the potential for applying ATHEANA beyond NPP settings. This paper provides a description of the process followed during the analysis, a description of the HFE scenario groupings, discussion regarding general human performance vulnerabilities, and a detailed examination of one HFE scenario developed in the study.

\section{INTRODUCTION}

Human reliability analysis (HRA) methods have been developed primarily to provide information for use in probabilistic risk assessments (PRAs) that analyze nuclear power plant (NPP) operations with particular emphasis on decision making in the control room. Despite this historical focus on the control room, there has been

\footnotetext{
${ }^{\text {a }}$ This work was funded by the U.S. Nuclear Regulatory Commission (USNRC) and performed at Sandia National Laboratories. Sandia is a multiprogram laboratory operated by Sandia Corporation, a Lockheed Martin Company, for the United States Department of Energy under Contract DE-AC04-94AL85000. The opinions expressed in this paper are those of the authors and not of the USNRC.
}

growing interest and discussion regarding the application of HRA methods to other NPP activities such as spent fuel handling $(\mathrm{SFH})$ or operations in different types of facilities. One recently developed HRA method, 'A Technique for Human Event Analysis' (ATHEANA) has been proposed as a promising candidate for diverse applications due to its particular approach for systematically uncovering the dynamic, contextual conditions influencing human performance. This paper describes one successful test of this proposition by presenting portions of a recently completed project in which a scoping study was performed to accomplish the following goals: (1) investigate what should be included in a qualitative HRA for spent fuel and cask handling 
operations; and (2) demonstrate that the ATHEANA HRA technique can be usefully applied to these operations. This analysis was performed without the benefit of the context provided by a larger PRA study, nor was it plant specific, and so it investigated only generic HRA issues relevant to SFH. However, the improved understanding of human performance issues provided by the study will likely enhance the ability to carry out a detailed qualitative HRA for a specific NPP at some point in the future. Furthermore, support was obtained regarding the potential for applying ATHEANA beyond NPP control room settings. This paper provides a description of the process followed during the analysis, a description of the human failure event scenario groupings, discussion regarding general human performance vulnerabilities, and a detailed examination of one HFE scenario developed in the study.

\section{DESCRIPTION OF THE ANALYSIS PROCESS}

The human performance analysis approach used in the work reported here was a qualitative, scoping level analysis conducted using elements of NUREG-1792 Good Practices for Implementing Human Reliability Analyses (HRA) [1] and NUREG-1624, Rev. 1, Technical Basis and Implementation Guidelines for A Technique for Human Event Analysis (ATHEANA) [2]. However, given the preliminary nature of this analysis, process descriptions, $\mathrm{HFEs}^{\mathrm{b}}$, unsafe actions ${ }^{\mathrm{c}}$ (UAs), and error forcing context ${ }^{\mathrm{d}}$ (EFC) descriptions were treated in a somewhat generic manner. In fact, although specific HFEs were generated, UAs, and EFCs were, in general, not explicitly identified during this qualitative HRA in order not to impose an excessive amount of structure on these preliminary scenarios. The resulting presentation of various scenarios and human performance considerations developed in the scoping analysis, while intentionally unconstrained to a specific HRA technique, were intended to serve as a good starting point for a more focused or plant specific state-of-the-art HRA analysis, including information gathering and HRA quantification activities.

Specific tasks that were conducted in support of this effort included:

- Identification and review of the literature on SFH and DCSOs (i.e., normal operations and

\footnotetext{
b 'Human failure events' are events that would be modeled as basic events in the logic models of a PRA, and that represent the failure of a function, system, or components that is the result of one or more unsafe actions.

c 'Unsafe actions' are actions inappropriately taken, or not taken when needed, by plant personnel that result in a degraded plant safety condition.

d 'Error-forcing contexts' are situations that arise when particular combinations of performance shaping factors and plant conditions create an environment in which unsafe actions are more likely to occur.
}

incidents) ranging from handling and storage of individual rods to handling and storage of spent fuel casks. Examples of the items reviewed include: analysis materials provided by the U.S. Nuclear Regulatory Commission and the Electric Power Research Institute (EPRI); the Final Safety Evaluation Report, Rev. 3, for the Holtec International HISTORM 100 cask system [3]; and NUREG-1774 A Survey of Crane Operating Experience at U.S. Nuclear Power Plants from 1968 through 2002 [4].

- Interviews with subject matter experts (SMEs) to investigate the details of SFH and DCSO activities including: human performance aspects, job aids ${ }^{\mathrm{e}}$, potential variations from 'typical' activities, and significant near misses and/or accidents that have occurred during these activities;

- Performance of an initial, scoping, qualitative, ATHEANA-type HRA of SFH and DCSO activities to discover opportunities where misloads and drops may occur, and to detail both how and why such events might occur given current understandings of human performance

The basic approach used in performing the scoping qualitative HRA was to separate the SFH and DCSO activities into HFE scenario groupings and then examine/explore the potential use or usefulness of job aids, plausible variations in context, potential error mechanisms for fuel handling-specific failures, and other performance shaping factors (PSFs) and vulnerabilities that may influence the likelihood and consequence of particular HFEs. The specific structure of the approach included the identification of a number of scenarios in which similar groups of human failure events may occur. Each HFE scenario grouping included a definition and interpretation of the issue being analyzed including a summary statement of the issue, the reason for the analysis, and the potential consequences should the issue materialize. In order to capture PSFs and vulnerabilities, without imposing an excessive degree of structure on the scenarios (i.e., to avoid undue bias toward a particular HRA method; however details beneficial for an ATHEANA application were generated), these items were grouped into 'general' human performance vulnerabilities - broadly applicable to an entire HFE group, and 'specific' human performance vulnerabilities associated with individual scenarios. The next two sections of this paper provide a description of the HFE scenario

\footnotetext{
e Job aids are repositories for information, processes, or perspectives; they are external to the individual; they support the work and activity to be done; they direct, guide, and enlighten performance (e.g., books, cards, software, alarms, control panels, various displays) [5].
} 
groupings, and then give an example of a scenario excerpted from one of the groupings, respectively.

\section{HUMAN FAILURE EVENT SCENARIO GROUPINGS}

A general description of fuel handling and cask operations was subdivided into categories of operations to facilitate logical grouping of HFEs and associated human performance vulnerabilities identified during the analysis. The categories that were developed represented a departure from previous groupings of operations as proposed in analysis materials provided by the USNRC and EPRI. The development of the following categories of operations was motivated by an attempt to effectively capture a wide range of human performance problems that may contribute to a misload and/or cask drop and to facilitate high-level comparisons of potential consequences ${ }^{\mathrm{f}}$ and risks associated with different cask systems. The SFH and DCSO categorization scheme used in the analysis was divided into the following seven phases of operation:

1. Fuel Load Planning -This phase of operation involves activities by the appropriate engineering department (e.g., nuclear fuels engineering) to generate a fuel move plan, incorporating proper review and approval with subsequent transmission to the fuel handlers who will carry out the operation. This activity depends upon proper

configuration management practices such that an accurate record of the history and specific location of every fuel assembly in the spent fuel pool (SFP) is maintained. The fuel movement plan should include the origin information-serial numbers and alphanumeric locations of assemblies within the SFP, and the destination information-cask canister locations and serial numbers of assemblies. In addition, the fuel load plan should include the process to be followed by fuel handling personnel during actual loading operations (e.g., 3-part communications, independent review of loaded canister before closure, etc.).

2. Cask Operations Personnel and Equipment Preparation -This phase of operation involves training and appropriate staffing of personnel for DCSOs as well as inspection, test, maintenance, recertification, upgrading, etc., of all structures, systems, and components that are required for executing DCSOs. An example of this phase would include assigning trained personnel or enabling proper training of personnel who then conduct detailed

\footnotetext{
${ }^{\mathrm{f}}$ Consequences were of particular interest in the preliminary scoping effort, although likelihood determination and risks (i.e., the product of consequences and likelihoods) were estimated to some degree in other analyses, the focus here was how a set of undesirable human actions might occur.
}

structural inspections of auxiliary or refueling building crane supports and interfacing building structures to insure that no cracks, deformations, or other aberrations threaten crane operations. This activity would be immediately accompanied with thorough inspection, test, and maintenance of crane systems and components before any critical heavy lifts are attempted (e.g., lifting a fuelloaded and water-filled cask from the spent fuel pool).

\section{Cask Preparation and Positioning -This phase of} operation represents the beginning of actual DCSOs as the cask is brought into the plant for loading preparation activities which culminate with the placement of the empty cask/canister system into the cask loading pit of the SFP in advance of fuel loading.

4. Cask Loading (esp. useful for consequence grouping)This phase of operation begins with placement of the first fuel assembly in the cask or canister and ends with the cask or cask and canister being properly drained, dried, inerted, and sealed.

5. Loaded Cask Transfer Within Structure (esp. useful for consequence grouping)-This phase of operation begins with preparations to transfer the loaded, sealed cask from the reactor, auxiliary, or fuel building and ends with the cask coupled to the cask transporter.

6. Loaded Cask Transfer Outside Structure (esp. useful for consequence grouping)-This phase of operation begins with a loaded cask, coupled to the cask transporter and ready for movement to the independent spent fuel storage installation (ISFSI) and ends with cask emplacement at the ISFSI.

7. Loaded Cask Storage and Monitoring (esp. useful for consequence grouping)-This phase of operation begins with cask emplacement at the ISFSI and ends when the cask contents (i.e., the spent fuel) are transferred to an off-site storage and/or processing location.

There are at least two major benefits of using the seven phases presented above. First, the inclusion of planning and preparation phases encourages more comprehensive analysis of the context of operations and events that can 'set-up' personnel for an accident in later phases ${ }^{\mathrm{g}}$. Therefore, a prospective analysis team may be more inclined to search for such 'latent' UAs when incorporating conceivable/credible HFEs into their analysis models. Second, the number of phases for

\footnotetext{
${ }^{g}$ On numerous occasions, HFEs (or near misses) that have actually occurred in nuclear power plants were preceded in time by UAs that were not anticipated during prospective human performance analyses and were often (at least initially) overlooked by post-incident/accident investigation teams [2].
} 
'direct ${ }^{\mathrm{h}}$ ' cask activities is generally expected to mirror the high-level 'hand-offs' that occur between teams of personnel. It is hoped that this categorization of operations (i.e., the seven phases) will be used to guide the analysis of human performance in any future, sitespecific DCSO PRA.

The seven phases of operation were used to group detailed descriptions of SFH and DCSO and to help guide the search process for potential HFEs and related human performance vulnerabilities. Seven grouping categories were then developed to separate logical, mid-level regions of conceivable/credible HFE scenarios that link to the taxonomy of operations. The HFE scenario descriptions were organized by the following seven HFE scenario grouping categories:

1. Scenarios before and during fuel loading

2. Scenarios during cask movement from spent fuel pool to preparation area

3. Scenarios during multipurpose canister (MPC) and transfer cask sealing operations

4. Scenarios during cask movement from preparation area to transfer pit

5. Scenarios during MPC movement from transfer cask down to storage cask

6. Scenarios during storage cask movement from transfer pit to ISFSI pad

7. Scenarios during monitoring and storage at the ISFSI

It should be recalled that HFEs are defined as events that would be modeled as basic events in the logic models of a PRA, therefore the categories above do not always map to the phases of operation in a one-to-one fashion. For example, the first category (i.e., scenarios before and during fuel loading) includes all of the planning and preparation operations, but also includes fuel loading since it is likely that this would be the first PRA-modeled operation $^{\mathrm{i}}$ available for a HFE. The $2^{\text {nd }}$ and $3^{\text {rd }} \mathrm{HFE}$ scenario grouping categories represent a subdivision of the $4^{\text {th }}$ phase of operation, and the $4^{\text {th }}$ and $5^{\text {th }}$ scenario grouping categories represent a subdivision of the $5^{\text {th }}$ phase of operation. The increasing detail for the HFE scenario groupings for those two phases of operation allows for a better logical separation of conceivable /credible HFEs. Furthermore, the specific terminology

\footnotetext{
${ }^{\mathrm{h}}$ In this instance 'direct' refers to hands-on activities that involve moving fuel, sealing casks, moving casks, etc. in contrast to 'indirect' activities involving planning, preparation, administration, etc. This 'direct' labor versus 'indirect' labor is common terminology in product manufacturing settings.

${ }^{\mathrm{i}}$ Given typical PRA practice, this could be considered the first operation in which a HFE is anticipated to potentially result a radiological incident or accident even though one or more UAs during previous operations may also contribute to the 'consequential' event.
}

(e.g., MPC, transfer pit, etc.) used in this particular HFE grouping strategy is intentionally biased toward the Holtec International HISTORM 100 cask system and a boiling water reactor plant design (this was a result of the specific information sources made available to the analysts). PRAs focused on different cask systems and/or plant designs would be expected to have slightly different HFE scenario grouping categories, while the cask operation categories would remain the same.

\section{GENERAL HUMAN PERFORMANCE VULNERABILITIES}

A mixture of inductive and deductive approaches were used to generate a listing of both general human performance vulnerabilities (applying to one or more scenarios in an HFE scenario grouping category) and specific human performance vulnerabilities unique to each scenario. To provide an indication of the types of vulnerabilities investigated, a small sample of general human performance vulnerabilities selected across several HFE scenario grouping categories is presented below:

Unchallenging Activities - The activities involved in spent fuel handling are, in general, quite simple in nature. In addition, the speed of the movements is quite slow, so each action takes a long time to complete. Basically, this is mostly boring work, and some individuals in the process have a significant amount of downtime between actions. There is ample opportunity for diversion and distraction, and an air of informality and complacency can easily exist within and amongst the team members. From a psychological perspective, there is potentially insufficient dynamic activity to generate an optimum stress/arousal level for performance. This lack of challenge, combined with high experience levels of personnel (i.e., they have performed these operations without incident many times) may lead to a progressive disregard for step-by-step procedures. Over time, a migration from strict adherence to step-by-step procedures, to occasional violations of procedures, to routine violations of procedures, may result in 'informal rules' that personnel accept as normal at some point in time.

Limited Indicators and Job Aids - Compared to the control panel and local indicators and other job aids that are common in the power plant operations, those that exist in spent fuel operations are quite limited. In general, processes are controlled primarily by visual cues.

Visual Challenges - As mentioned above, visual cues are the primary means of performing spent fuel operations. In many cases, properly observing these cues is made difficult by the positioning of people in relation to the 
activities being observed. Operations within the spent fuel pool can be particularly challenging, as the effect of refraction in the water and reflection from the surface of the water can distort the view of operations that require precise positioning. Observing signs of damage to individual fuel pins within a cask or canister may be severely hampered by structural elements.

Crane operations have challenges whether they are in the water or not. The crane operator may need to lean out over the crane bridge as the view of an operation is essentially only from directly above. Many of the potential errors that could occur are related to vertical position, which cannot be determined from above. In addition, even the view from above may be obstructed, either by the yoke or by the load being moved. Thus, the operator is often put in the position of being the hands for someone else's eyes, which make the operations vulnerable to the communication vulnerabilities discussed below.

Finally, in many cases the action being viewed, by its very nature and location, must be viewed from a distance. In such cases, small deviations that could possibly lead to significant problems can be missed simply because of the inability to have sufficient resolution to detect the error.

Communication Difficulties - There are significant challenges in communication between the team members performing spent fuel operations. The environment contains a significant amount of background noise, predominantly machine noise. Although headsets are used by key participants for communication, they do not eliminate the potential for misunderstanding. Garbled communication (due to system interference or background noise) is clearly possible, and in some cases it may not even be possible to clearly determine who is speaking. A belief that a particular individual is speaking, even if they are not, can bias the listener into hearing what he expects to hear.

Time pressure - Although time pressure during cask loading campaigns (CLCs) is generally less than during refueling outages (due to the non-producing status of the plant during an outage), missing scheduled milestones can lead to increased expenses and increased uncertainty with regard to time schedules for upcoming outages. SMEs have stated that time pressure can quickly emerge, even during fuel assembly movement operations. This ability for time pressure to emerge may be exacerbated by the perception of low consequence for errors during this process. All personnel perceive the dropping of a very large cask from a crane to create high consequence outcomes; therefore those operations are much less susceptible to time pressure. Handling of individual fuel assemblies may not carry with it the same need for slow, step-by-step execution. The tone set by all levels of management regarding the relative goods of ensuring safety versus meeting a predetermined schedule will greatly impact the perception of time pressure among operations personnel.

Other Ergonomic Issues - Additional stressors include the cramped working space on the refueling crane bridge and those related to clothing (i.e., the suits required when working above the spent fuel pool).

Configuration control - Configuration control processes are not always designed to avoid specific human performance problems that may arise due to design peculiarities at a specific site. Such processes are driven by the accumulation of knowledge and experience by those who administer/manage the system, but thorough documentation of such knowledge and experience, which influences the assumptions and error checking processes used during activities, may not be present. These omissions can lead to problems (e.g., improperly prepared fuel movement plans) when a hand-off of configuration control activities to new personnel occurs.

Trust - Trust is an essential component of any team-based activity. Crew members must be able to depend upon the correct behaviors of others when performing operations. However, trust can have a negative component as well. An example is provided in one scenario where a supervisor 'trusts' his experienced FHP and spotter leading to a cursory verification of fuel assembly loading. Crew members must always be reminded of the proper orientation of the 'trust' relationship. In this case, trust should imply that the FHP and spotter can 'trust' the supervisor to carefully review the fuel load to protect them from missing errors that will be sealed inside the cask and may present hazards to others years later.

\section{EXAMPLE OF A HUMAN FAILURE EVENT SCENARIO}

The work that is briefly described in this paper included a number of preliminary scenarios developed for SFH and DCSOs. These preliminary scenarios included human failure events that might be modeled in a plant-specific PRA, although they were generated from a non-plant specific information base that was notably impoverished relative to a "complete" set that would be expected for a full HRA/PRA analysis. Since previous analyses were reviewed during the performance of this preliminary effort, some of the scenarios contain human failure events that were addressed to some degree in those previous studies. However, it is important to note that none of the previous analyses provided a thorough investigation of the contexts (i.e., an ATHEANA-like approach) in which failures may occur. Therefore, even for HFEs identified in 
previous studies, this analysis provided more insight and enables more understanding of how those HFEs may actually occur. In addition, there was no attempt to be exhaustive in the search for possible scenarios, but rather it was deemed sufficient for scoping and demonstration purposes to cover a broad spectrum of scenario examples.

The example HFE scenario given in this section is taken from the $3^{\text {rd }}$ HFE scenario grouping category (i.e., Scenarios during multipurpose canister (MPC) and transfer cask sealing operations). The introductory, context-setting material for this scenario grouping category (containing five scenario descriptions) is provided first and is followed by the first scenario description titled: Failure to identify a fuel misload event.

The phase of operation related to this HFE scenario grouping category begins with the loaded transfer cask resting in the proper position in the preparation area for closure operations with the scaffolding properly arrayed around the outside of the transfer cask. It continues through sealing, purging, drying, and inerting operations. This phase ends when both the MPC and transfer cask are ready to be transported to where the MPC will be inserted into the storage cask.

1. Definition and interpretation of issue being analyzed

a. Human failure event scenarios during MPC and transfer cask sealing operations - In this process the MPC is loaded, with the MPC lid placed on top and the MPC is resting inside the transfer cask at the preparation area. All of the closure and preparation activities are performed such that the MPC becomes ready for emplacement in the shielded storage cask.

b. Reason for analysis - These scenarios are being analyzed due to the potential for identifying a fuel misload event, a human initiated fire event, and most importantly, for the potential to leave a leak path, or a 'soon to be present' leak path condition from the inside of the MPC to the outside of the MPC.

c. Potential consequences - Storing misloaded fuel may result in a degradation of fuel assemblies such that fission products migrate to the general environment within the MPC; a human initiated fire during closure operations may create a condition that leads to fuel damage and a release of fission products to the reactor, auxiliary, or fuel building environment; and the establishment of a leak path could allow for fission product migration to the storage cask or module at the ISFSI, which may then migrate away from the ISFSI and pose a threat to the public and the environment.

2. Base case scenario
Initial conditions - The initial conditions for the start of this phase of operation will vary with the specific plant and cask system being used. A typical situation is defined:

- $\quad$ The loaded MPC in the transfer cask is positioned properly in the preparation area with scaffolding also properly positioned around the cask.

- The MPC lid has been placed into position, but is merely resting, unsecured on the MPC shell.

- Personnel are decontaminating the area around the top flange of the transfer cask and getting ready to install the temporary shield ring or other form of gamma radiation shielding to prevent radiation streaming from the trunnion recess areas of the transfer cask water jacket.

\section{General Human Performance Vulnerability Concerns}

Provided below is a brief summary of some potential human performance vulnerabilities that may impact MPC and transfer cask sealing operations:

Decision making biases based on perception of loss - The manner in which a person frames the concept of 'loss' in a given situation provides a strong biasing factor toward all actions that enable the person to steer away from incurring that 'loss' [6]. People often tend toward the discovery of a simple, non-loss threatening alternative explanation to a situation, instead of attending to a complex, loss-threatening explanation to a situation. For example, the situation in which a radiation protection (RP) person detects high radiation levels after a redecontamination of the lid enables him to choose the nonloss threatening explanation of "I just swiped too close to a normally 'hot' area" as opposed to the loss-threatening explanation of "Oh, no, we've got misloaded fuel in here and need to spend considerable time and effort to get the cask back in the pool and thoroughly investigate. Not only that, but we better move quickly." The losses referred to here are the loss of time, lost of respect for those who 'messed up and got us into this situation', and potential loss related to damaging fuel that then leads to a fission product release. Another example of this loss avoidance behavior is that of personnel draining, purging, drying, and backfilling who choose the simple, non-loss threatening explanation of a 'welding delay' leading to excessive temperatures (the specifics of this example are elaborated on in scenario 1 in this section).

Interestingly, at the point in the future when the fuel misload condition described in scenario 1 is eventually discovered, the incident investigators will probably be astounded that multiple personnel disregarded signs of a fuel misload, as the potential consequences of fuel damage, fission product release, etc. are much higher than any 'mere inconvenience' of getting the fuel back in the 
pool and carefully tending to a potential problem. Of course, for the personnel conducting the tasks 'in the moment' (e.g., under some level of time pressure, not wanting to disrupt major operations and schedules, not wanting to tarnish the team's reputation, etc.) the mental accounting of 'loss' may allow them to filter out and explain away signals that point to a misload event. Personnel 'in the moment' may be thinking that they don't want to be forced to deal with a misload event and they also can't really imagine that the many barriers against a misload event would somehow be circumvented ${ }^{\mathrm{j}}$. Readers wanting to learn more about the interesting, important, and complex topic of loss avoidance and how people may conceptualize or perceive real/potential losses are encouraged to review references 6-8.

Limited Nature of Procedures - The cask sealing operations may be relatively well proceduralized, but they still depend primarily on skills learned and additional training experiences. In these activities, procedures specify basic tasks in the process, but a number of skillbased sub-tasks are performed at the discretion of particular individuals and teams. Examples of specific, potential procedural oversights in SFH and DCSOs may include: CLC preparations not accounting for potential rapid relocation of fire ignition sources to flammable material storage areas due to rapid air movement by the HVAC system. This may lead to improper designation of 'safe' areas for flammable items and stationing of fire fighting personnel during 'ignition prone' operations (e.g., welding, grinding, or cutting torch operations). Second, a lack of explicit procedures specifying that both members of a cask closure team must inspect all bolt holes for the presence of water. Procedures reviewed during the analysis only specified that all bolt holes needed to be visually inspected; therefore an opportunity for quality assurance redundancy may be missed.

Time of day and shift work - Many of the scenarios reveal an all too common pattern that emerges in shift work situations. Slips, lapses, mistakes, and violations tend to occur more often when workers are fatigued, especially when that fatigue is encountered during late

\footnotetext{
${ }^{\mathrm{j}}$ It should be noted that the authors of this paper are not trying to imply that such a fuel misload event scenario is somehow highly likely; such scenarios are simply designed to plausibly argue how an occurrence deemed 'highly unlikely' using certain analysis techniques, may actually happen when human beings play crucial roles in the process. It should also be noted that one of the authors of this report has devised a framework which may provide assistance in detecting and mitigating parts of an operation that are vulnerable to undesirable actions based on the mental accounting of real or potential 'loss' in addition to many other associated perceptual/decision making biases (e.g., confirmation bias, etc.) [6].
}

night or early morning hours ${ }^{\mathrm{k}}$. Furthermore, personnel working occasional night shifts may be tempted to rush operations in order to end shifts early or at least change the focus to non taxing activities (e.g., hurry up with the welding that demands significant mental and physical effort, and then leisurely finish the balance of the shift with minimal effort tasks of tear-down and clean up activities).

Pace of Operations - Some of the activities involved in cask sealing, drying, purging, backfilling operations are, in general, quite simple in nature. In addition, the speed of many of the movements is quite slow, so each action takes a long time to complete. Basically, this can be boring work, and some individuals in the process have a significant amount of downtime between actions. There is ample opportunity for diversion and distraction, and an air of informality and complacency can easily exist within and amongst the team members. From a psychological perspective, there may be insufficient dynamic activity to generate an optimum stress/arousal level for performance.

Visual Challenges - As mentioned above, visual cues are the primary means of performing cask closure operations such as cleaning, grinding, tack welding, and even liquid dye penetrant testing and hydrostatic testing. Maintaining visual vigilance for long periods is difficult work 1 .

Ultrasonic testing is often not suited for the major lid and closure ring welds, therefore, visual and tactile cues are critical. Another specific visual challenge exposed in the example scenario below comes from the use of a non-auto darkening welding helmet which reduces the ability of the welder to rapidly detect ignition of flammable material following a hydrogen ignition during lid closure operations.

Other Ergonomic Issues - Additional stressors include the cramped working space around the transfer cask and heat stress due to ambient temperatures and those related to clothing (i.e., the contamination control suits and additional clothing for protection from welding slag).

\footnotetext{
${ }^{\mathrm{k}}$ It is possible that not all individuals are affected this way as a function of late night or early morning activities, but the general stereotype has proven to be very strong, and despite some directed efforts, there has not been a reliable way to identify/select people who are especially suited to night shifts or early morning shifts (i.e., within subject variability is not well-understood). Day-to-night physiological changes (i.e., circadian rhythms) are well documented in a many studies of human performance. See reference [9] for an introduction and overviews of the circadian rhythm and shift work literature.

${ }^{1}$ Unpublished research at Sandia National Laboratories has recently discovered dramatic levels of omission during aircraft structural inspections among highly experienced, highly motivated maintenance personnel. When confronted with the results of these experiments, many of the maintenance personnel are shocked to discover their actual level of performance. For discussion on human signal detection and for entry points into the extensive literature on this topic, see references [9-11].
} 
A number of actual events involving incomplete or incorrect procedures during sealing operations and also of actual hydrogen ignition events during lid sealing have occurred as documented in the NEI database on Spent Fuel Handling events. Summaries of those events were made available to the authors via a CD from the NRC. Specific events are not listed here as they were generally events of minor consequence. The point made here is that slips, lapses, mistakes, and violations have occurred during these types of operations.

\section{Example of a Scenario Description within the $3^{\text {rd }}$ HFE Scenario Group}

This section provides a high-level overview of the five scenarios that were developed for the $3^{\text {rd }}$ HFE scenario group titled: Scenarios during multipurpose canister $(M P C)$ and transfer cask sealing operations. Table 1 briefly lists the five potential human failure event scenarios and associated human performance vulnerabilities identified in the $3^{\text {rd }}$ HFE scenario group. It is important to note that not every one of the listed vulnerabilities apply to each of the scenarios. The first scenario description within the $3^{\text {rd }}$ HFE scenario group, which is the example included in this paper, is titled: failure to identify a fuel misload event.

Table 1. Scenarios during MPC and transfer cask sealing operations.

\begin{tabular}{|c|c|c|c|}
\hline $\begin{array}{c}\text { HFE } \\
\text { Group }\end{array}$ & $\begin{array}{l}\text { HFE Group } \\
\text { Description }\end{array}$ & Scenario & Vulnerabilities \\
\hline 3 & $\begin{array}{l}\text { MPC and } \\
\text { transfer cask } \\
\text { sealing } \\
\text { operations. }\end{array}$ & $\begin{array}{l}\text { 1. Failure to identify a fuel } \\
\text { misload event } \\
\text { 2. Human initiated fire } \\
\text { event-welded cask } \\
\text { 3. Failure leaves leak path } \\
\text { existing at the end of } \\
\text { sealing and preparation } \\
\text { activities-welded cask } \\
\text { 4. Failure leads to impending } \\
\text { leak path due to undetected } \\
\text { problem during sealing and } \\
\text { preparation } \\
\text { activities-welded cask } \\
\text { 5. Failure leads to impending } \\
\text { leak path due to undetected } \\
\text { problem during sealing and } \\
\text { preparation } \\
\text { activities-bolted cask }\end{array}$ & $\begin{array}{l}\text { - Biases based on perception } \\
\text { of loss } \\
\text { - Limited nature of } \\
\text { procedures } \\
\text { - Time of day \& shift work } \\
\text { - Pace of operations } \\
\text { - Visual challenges } \\
\text { - Perceived time pressure } \\
\text { Omission in hazard } \\
\text { analysis } \\
\text { - Improper training } \\
\text { - Overconfidence } \\
\text { - Oapse } \\
\text { Other ergonomic issues } \\
\text { (welder's helmet) }\end{array}$ \\
\hline
\end{tabular}

5. Description of the example scenario: Failure to identify a fuel misload event

The example below begins with a description of the sequential operations performed in the scenario, and finishes with a list of potential human vulnerabilities specific to the scenario.

Preparation worker does not decontaminate lid properly - The preparation worker (i.e., the individual responsible for decontamination of the MPC lid surface and top flange area) does not completely wipe down and decontaminate the MPC lid and top flange area. The specific area that is not wiped down completely is near where the shield ring is installed to absorb gamma radiation near the trunnion recess areas of the transfer cask. The omission occurs as the preparation worker is trying to work around other personnel preparing to move the shield ring into position.

Radiation protection worker detects high radiation levels - After the shield ring is installed, the radiation protection (RP) worker detects an unusually high level of radiation emitting from the area that was not properly decontaminated above the trunnion recess area. The preparation worker who is standing next to the RP workers recalls that he forgot to wipe down that area and mentions that fact to the RP worker. The preparation worker then wipes down the area. The RP workers makes another pass with the radiation monitor and finds a lower, but still unusually high level of radiation. He readily dismisses the radiation level to moving the probe too close to the trunnion recess side of the shield ring.

Welding equipment delay - A problem with the automated welding equipment causes a delay that postpones closure operations for more than an hour.

Excessive temperatures during draining and purging are attributed to delay - Unusually high MPC internal temperatures noted during the draining and purging processes are attributed to the delays in getting the cask sealed. Even some indications of localized water boiling in the cask are not investigated thoroughly. That is, personnel are expecting the cask to heat up due to fuel decay heat when the MPC is filled with non-circulating, non-cooled water. The personnel are encouraged to keep moving and get the vacuum process under way as that will remove a large amount of heat from the MPC.

Lack of evidence of excessive cooling in the vacuum lines is positively received by personnel - Typically, a gradual step process is used when lowering MPC pressure using the vacuum drying system, this is often necessary to prevent ice from forming in parts of the system. With the present 'warm' cask, evidence of icing never occurs and the preparation personnel just continue with a rapid evacuation process and subsequent helium backfill.

Early pressurization with helium is not noticed - The personnel backfilling the cask with helium did not carefully estimate how much gas should be required to pressurize the MPC (e.g., from past experience, or from rough phenomenological calculations); therefore, they did not notice that it required a significantly smaller volume of helium to reach required pressures in the cask. Welding of remaining cask penetrations was completed quickly after the backfill and an increased MPC internal pressure was not discovered. 
Potential Human Performance Vulnerabilities for which may facilitate the human actions that lead to the realization of the scenario 1 human failure event includes the following:

- Lack of detailed procedures without appropriate thresholds for alarm

- Equipment calibration errors

- Perceived time pressure

- The ease of finding a simple, non-loss threatening alternate explanation to a situation, instead of attending to a complex, loss-threatening explanation to a situation. Examples: the RP person detecting and explaining away high radiation levels after the redecontamination of the lid; the draining, purging, drying, and backfilling personnel who choose the simple, non-loss threatening explanation of a 'welding delay' leading to excessive temperatures.

\section{CONCLUSIONS}

ATHEANA has been proposed as a promising candidate for diverse applications due to its particular approach for systematically uncovering the dynamic, contextual conditions influencing human performance. This paper described one successful test of this proposition by presenting portions of a recently completed project in which a scoping study was performed to accomplish the following goals: (1) investigate what should be included in a qualitative HRA for spent fuel and cask handling operations; and (2) demonstrate that the ATHEANA HRA technique can be usefully applied to these operations. This paper provided a description of the process followed during the analysis, a description of the HFE scenario groupings, discussion regarding general human performance vulnerabilities, and a detailed examination of one HFE scenario developed in the study. Although the preliminary analysis was generic and performed without the benefit of the context provided by a larger PRA study, it is argued that the improved understanding of human performance issues provided by the study will likely enhance the ability to carry out a detailed qualitative SFH and DCSO HRA for a specific NPP at some point in the future. Furthermore, support was obtained regarding the potential for applying ATHEANA beyond NPP control room settings.

\section{REFERENCES}

[1] NUREG-1792, “Good Practices for Implementing Human Reliability Analysis,” U.S. Nuclear Regulatory Commission, Washington, DC, April 2005.

[2] NUREG-1624, Rev. 1, "Technical Basis and Implementation Guidelines for A Technique for
Human Event Analysis (ATHEANA), U.S. Nuclear Regulatory Commission, May 2000.

[3] Holtec International, "Holtec International Final Safety Analysis Report for the HI-STORM 100 Cask System, Revision 3, Report no. HI2002444," Holtec International, Marlton, NJ, 2005.

[4] NUREG-1774, “A Survey of Crane Operating Experience at U.S. Nuclear Power Plants from 1968 through 2002," U.S. Nuclear Regulatory Commission: Washington, DC., 2003.

[5] Rossett, A. and J. Gautier-Downes, “A Handbook of Job Aids," Pfeiffer \& Company, San Diego, CA., 1991.

[6] Brewer, J. D., "Risk Perception \& Strategic Decision Making: General Insights, a New Framework, and Specific Application to Electricity Generation Using Nuclear Energy, SAND2005-5730," Sandia National Laboratories, Albuquerque, NM., 2005. Kahneman, D. and A. Tversky, "Prospect Theory: An analysis of decision under risk," Econometrica, 47(2): 263-291., 1979.

[8] Kahneman, D. and A. Tversky, "Choices, Values, and Frames," American Psychologist, 39(4): 341-350., 1984.

[9] Salvendy, G. ed., Handbook of Human Factors and Ergonomics, John Wiley \& Sons, New York, NY., 1997.

[10] Wickens, C. D. and J. G. Hollands, "Engineering Psychology and Human Performance, 3rd edition," Prentice Hall, Upper Saddle River, NJ., 2000.

[11] Kantowitz, B. H. and R. D. Sorkin, "Human Factors: Understanding People-System Relationships," John Wiley \& Sons, New York, NY., 1983. 
APPENDIX E 


\section{APPENDIX A \\ EXPERT ELICITATION BACKGROUND INFORMATION WORKSHEET}

Instructions. Complete this worksheet prior to contacting the expert. Provide this worksheet and supporting materials to present the problem domain to the expert. All experts should receive identical information.

1. Analyst's Name and NRC Affiliation:

2. Problem Type: $\quad \square$ Actual Hardware Failure

$\square$ Latent Hardware Failure $\quad \square$ Other:

$\square$ Actual Human Error

$\square$ Latent Human Error

3. Summary of Problem for Analysis:

4. Supporting Documents (Attached):

5. Summary of Results from Initial Analysis:

6. Analysis Assumptions:

7. Information Required from Expert:

A-1 
A-2

272 


\section{APPENDIX B \\ EXPERT ELICITATION WORKSHEET FOR HARDWARE FAILURE}

Instructions. Complete this worksheet for each individual expert. Begin by answering any questions the expert has regarding the problem being analyzed. Then step through each question in sequence. Attach any supporting materials provided by the expert.
1a. Date of Elicitation:
I 1
1b. Time of Elicitation:
$\square$ A.M. / $\square$ P.M.

2. Expert's Name and Affiliation:
$\square$ In-house NRC
$\square$ Industry Consultant
$\square$ Academia
$\square$ Other:
$\square$ National Lab/DOE
$\square$ Licensee
$\square$ Vendor

3. Expert's Areas of Expertise Relevant to Analysis:

4. Expert's Comments on Problem Under Analysis:

5. Median Failure Rate/ Percent of Time There's a 50/50 Likelihood of Hardware Failure:

50th Percentile Value (Median)

6. Upper Bound/Percent of Time That Hardware Will Almost Certainly Fail:

7. Factors Shaping Expert Estimate:

8. Additional Comments by Expert:

B-1 
B-2

274 


\section{APPENDIX C}

\section{EXPERT ELICITATION PANEL WORKSHEET FOR HARDWARE FAILURE}

Instructions. Complete this worksheet for the expert panel and data aggregation. Follow instructions in the guideline for facilitating the discussion. Begin by explaining the purpose of the panel, with a goal toward sharing information and arriving at a consensus. Next, read each expert's estimation. Provide the initial aggregation of expert estimates in 3 below. Allow 5 - 10 minutes for questions and another 10 - 15 minutes for discussion. Allow 5 minutes for final discussion and consensus. Allow the experts to modify their individual Worksheet $B$ to incorporate any new information from the discussion.

1a. Panel Conducted? $\square$ Yes/ $\square$ No 1b. Reason:

1c. If NO, Skip to 5

2a. Date of Panel: I

2b. Time of Panel:

$\square$ A.M. / $\square$ P.M.

3. Mean of Experts' Median and 95th Percentile Values

4. Summary of Main Points and Issues Raised in Discussion (Including Areas of Disagreement):

5. Consensus Estimate (Within $3 x$ for Median)? $\quad$ Yes $/ \square$ No

6a. If YES, Record Median of Median and 95th Percentile Values

6b. If NO, Record Mean of Median Estimates and 95th Percentile Values

7. Record Alpha $(\alpha)$ and Beta $(\beta)$ Values Derived from $6 a$ or $6 \mathrm{~b}$ for

Beta Distribution or Other Parameters for Non-Beta Distribution:

C-1 
C-2

276 


\section{APPENDIX D}

\section{EXPERT ELICITATION PANEL WORKSHEET FOR SPAR-H}

Instructions. Complete this worksheet for the SPAR-H expert panel and data aggregation. Follow instructions in the guideline for facilitating the discussion. Begin by explaining the purpose of the panel, with a goal toward sharing information and arriving at a consensus. Next, review each expert's SPAR-H worksheets. Discuss PSFs and dependency assumptions that differ between experts. Allow $5-10$ minutes for questions and another $10-15$ minutes for discussion. Allow 5 minutes for final discussion and consensus. Allow the experts to modify their individual SPAR-H worksheets to incorporate any new information from the discussion.

1a. Panel Conducted? $\square$ Yes $/ \square$ No 1b. Reason:

1c. If NO, Goto 4.

2a. Date of Panel: __ _ _

3. Summary of Main Points and Issues Raised in Discussion Regarding PSF and Dependency Assignments:

4. Experts Agree on a Single SPAR-H HEP Value? $\square$ Yes / $\square$ No

5a. If YES, Record HEP Constrained Noninformed Prior Distribution.

5b. If NO, Record Mean HEP _ and on the Next Sheet, Record the Distribution of PSF Assignments Across Experts. This Distribution May be Inserted into SAPHIRE.

D-1 
5c. Record Distribution of PSF Assignments Across Experts for Diagnosis and Action SPAR-H Worksheets. Record the Percentage of Experts Who Assigned Each Level.

DIAGNOSIS

\begin{tabular}{|c|c|c|}
\hline PSFs & PSF Levels & $\begin{array}{l}\text { Percentage } \\
\text { Selected }\end{array}$ \\
\hline \multirow{6}{*}{$\begin{array}{l}\text { Available } \\
\text { Time }\end{array}$} & Inadequate time & \\
\hline & $\begin{array}{l}\text { Barely adequate time }(\approx 2 / 3 \\
x \text { nominal) }\end{array}$ & \\
\hline & Nominal time & \\
\hline & $\begin{array}{l}\text { Extra time (between } 1 \text { and } \\
2 \times \text { nominal and }>\text { than } 30 \\
\text { min) }\end{array}$ & \\
\hline & $\begin{array}{l}\text { Expansive time }(>2 x \\
\text { nominal and }>30 \mathrm{~min})\end{array}$ & \\
\hline & Insufficient information & \\
\hline \multirow{4}{*}{$\begin{array}{l}\text { Stress/ } \\
\text { Stressors }\end{array}$} & Extreme & \\
\hline & High & \\
\hline & Nominal & \\
\hline & Insufficient Information & \\
\hline \multirow[t]{5}{*}{ Complexity } & Highly complex & \\
\hline & Moderately complex & \\
\hline & Nominal & \\
\hline & Obvious diagnosis & \\
\hline & Insufficient Information & \\
\hline \multirow{4}{*}{$\begin{array}{l}\text { Experience/ } \\
\text { Training }\end{array}$} & Low & \\
\hline & Nominal & \\
\hline & High & \\
\hline & Insufficient Information & \\
\hline \multirow[t]{6}{*}{ Procedures } & Not available & \\
\hline & Incomplete & \\
\hline & Available but poor & \\
\hline & Nominal & \\
\hline & $\begin{array}{l}\text { Diagnostic/symptom } \\
\text { oriented }\end{array}$ & \\
\hline & Insufficient Information & \\
\hline \multirow{5}{*}{$\begin{array}{l}\text { Ergonomicst } \\
\text { HMl }\end{array}$} & Missing/Misleading & \\
\hline & Poor & \\
\hline & Nominal & \\
\hline & Good & \\
\hline & Insufficient Information & \\
\hline \multirow{4}{*}{$\begin{array}{l}\text { Fitness for } \\
\text { Duty }\end{array}$} & 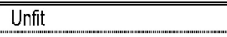 & \\
\hline & Degraded Fithess & \\
\hline & Nominal & \\
\hline & Insufficient Information & \\
\hline \multirow{4}{*}{$\begin{array}{l}\text { Work } \\
\text { Processes }\end{array}$} & Poor & \\
\hline & "Nominal & \\
\hline & Good & \\
\hline & Insufficient Information & \\
\hline
\end{tabular}

\section{ACTION}

\begin{tabular}{|c|c|c|}
\hline PSFs & PSF Levels & $\begin{array}{l}\text { Percentage } \\
\text { Selected }\end{array}$ \\
\hline \multirow{6}{*}{$\begin{array}{l}\text { Available } \\
\text { Time }\end{array}$} & Inadequate time & \\
\hline & $\begin{array}{l}\text { Time available is } \approx \text { the time } \\
\text { required }\end{array}$ & \\
\hline & Nominal time & \\
\hline & $\begin{array}{l}\text { Time available } \geq 5 x \text { the } \\
\text { time recuired }\end{array}$ & \\
\hline & $\begin{array}{l}\text { Time available is } \geq 50 x \text { the } \\
\text { time reculred }\end{array}$ & \\
\hline & Insufficient Information & \\
\hline \multirow{4}{*}{$\begin{array}{l}\text { Stress' } \\
\text { Stressors }\end{array}$} & Extreme & \\
\hline & High & \\
\hline & Nominal & \\
\hline & Insufficient Information & \\
\hline \multirow[t]{4}{*}{ Complexity } & Highly complex & \\
\hline & Moderately complex & \\
\hline & Nominal & \\
\hline & Insufficient Information & \\
\hline \multirow{4}{*}{$\begin{array}{l}\text { Experience/ } \\
\text { Training }\end{array}$} & Low & \\
\hline & Nominal & \\
\hline & High & \\
\hline & Insufficient Information & \\
\hline \multirow[t]{5}{*}{ Frocedures } & Not available & \\
\hline & Incomplete & \\
\hline & Available, but poor & \\
\hline & Nominal & \\
\hline & Insufficient Information & \\
\hline \multirow{5}{*}{$\begin{array}{l}\text { Ergonomics/ } \\
\text { HMI }\end{array}$} & Missing/Misleading & \\
\hline & Poor & \\
\hline & Nominal & \\
\hline & Good & \\
\hline & Insuff cient Information & \\
\hline \multirow{4}{*}{$\begin{array}{l}\text { Fithess for } \\
\text { Duty }\end{array}$} & Unfit & \\
\hline & Degraded Fitness & \\
\hline & Nominal & \\
\hline & Insufficient Information & \\
\hline \multirow{4}{*}{$\begin{array}{l}\text { Work } \\
\text { Processes }\end{array}$} & Poor & \\
\hline & Naminal & \\
\hline & Good & \\
\hline & Insufficient Information & \\
\hline
\end{tabular}

5d. If No Consensus Reached on Dependency Assignments, Note Source of Differences and Record Mean HEP from $5 b$. 


\section{APPENDIX E}

\section{EXPERT ELICITATION CHECKLIST}

Instructions. Complete this worksheet to ensure successful completion of all steps in the expert elicitation process. Internal or public release of an expert elicitation using this guideline assumes the analyst has successfully completed this checklist, and it is therefore not necessary to include this checklist. However, if the analyst has not completed all steps in this process, any deviations must be fully disclosed.

1. Entry Conditions for Expert Elicitation Met:

$\square$ Additional sources of information consulted and found inadequate to inform probability

$\square$ Event is risk significant

$\square$ Event is new, rare, complex, or otherwise poorly understood

2. Frame the Problem:

$\square$ Worksheet A completed

$\square$ Supporting documentation included

3. Experts Identified:

$\square$ Use of at least 2 experts

$\square$ Care taken to avoid biased sample of experts

4. Conduct Estimation:

$\square$ Worksheet B or SPAR-H Worksheet completed for each expert

$\checkmark$ Supporting documentation from experts provided (if any)

5. Expert Panel:

$\square$ Expert panel held $\quad$ or $\quad \square$ Expert panel not necessary due to consensus

$\square$ Summary of arguments

noted in Worksheet C

(hardware failure) or $D$

(human error)

6. Aggregation:

$\square$ Aggregation consensus or calculations noted in Worksheet $C$ (hardware failure) or $D$ (human error)

7. Risk Analysis Incorporation:

$\square$ Expert estimate successfully incorporated into PRA model

8. Note any deviations from the requirements in 1 - 7 and document in final analysis writeup.

\section{E-1}


APPENDIX F 
Ch. 20. Tables of Estimated HEPs

Overview

CHAPTER 20. TABLES OF ESTIMATED HUMAN ERROR PROBABILITIES

\section{Overview}

This chapter summarizes the estimated human error probabilities (HEPs) and their uncertainty bounds (UCBs) (or error factors [EFs]) presented in Part III. The tables in this chapter are duplicates of data tables in Part III except for changes to footnotes and table references to make them appropriate to Chapter 20. Not all data tables in Part III are included in this chapter; those that are included are sufficient for most human reliability analyses (HRAs) conducted as part of a probabilistic risk assessment (PRA). These tables are intended for use as quick references and are cross-referenced to the chapters from which they are drawn. The user is urged to familiarize himself with the source chapters for the proper use of the error terms and the assumptions on which they are based.

This chapter begins with a brief discussion of performance shaping factors (PSFs), followed by a search scheme for the use of the tables, with an explanatory talk-through of the search scheme. The chapter concludes with a list of tables, a quick-reference guide to the tables, and the set of tables.

For users conducting HRAs, the search scheme provides guidance to the appropriate tables at each stage of the analysis. The quick-reference guide is intended for general use and will help the analyst locate any table of interest.

\section{Performance Shaping Factors}

All of the estimated HEPs in the data tables are nominal HEPs, $i . e .$, they represent HEPs before plant-specific PSFs have been taken into account. When these latter are evaluated, a nominal HEP may be modified upward or downward.

Chapter 3 describes the usual PSFs that influence HEPs in industrial settings. PSFs specific to classes of activities are discussed in detail in Part III. As a rule, the HEPs in the Handbook are based on "average" industrial conditions. We define average industrial conditions as those that do not subject a worker to an unusual degree of discomfort and that are fairly representative of the industry. The user may modify the tabled HEPs if the PSFs for his specific application are not average. Some guidance is given to help the analyst to determine the average conditions applicable to each group of HEPs, but most of this information is presented in Part III.

PSFs such as temperature, noise level, lighting, and others related to the comfort or health of the worker will usually be average (or better) in nuclear power plants (NPPs). This is because regulatory agencies such as the Nuclear Regulatory Commission and the occupational Safety and Health Administration have developed "guidelines" or "recommended limits" for most controllable factors affecting workers. The plants' managements will work 
to meet the standards set by such agencies, and organizational units such as employee unions and professional organizations will usually report any deviations from these standards.

The PSFs related to ergonomics considerations are not subject to regulation. Hence, considerable variations exist from plant to plant as well as within any given plant. The estimated HEPs summarized here are based on conditions observed in a number of operating U.S. and foreign plants. In some cases, differences in PSFs have been estimated in the breakdown of the HEPs. For example, modifications to HEPs based on the PSFs of display type and information displayed have been defined in the data tables. Display types such as analog neters, digital indicators, chart recorders, etc., have been analyzed for the effect they have on human performance; the HEPs for errors made in dealing with displays have been modified to account for these effects. Very small differences in performance that might result from relatively minor differences in human factors engineering of displays, e.g., indicator needle length and width, are not represented in the estimated HEPs.

In other cases, it is not possible to provide quantitative estimates of substantial differences in levels of a PSF. For example, for the PSF of the quality of administrative control, the user will have to be content with rating this PSF as "good," "average," or "poor," making a subjective decision about the effect of this PSF on any particular task. Guidance is given for evaluating the effects of these types of PSFs, but considerable judgment by the analyst will be required.

The UCBs (or EFs) for an HEP reflect the estimated range of variability in performance attributable to differences in relevant PSFs, differences between and within people, differences in analysis, modeling uncertainty, and uncertainty about the actual HEPs. The tabled UCBs are speculative; the analyst may wish to expand them to indicate greater uncertainty. The tables Iist the EFs or UCBs for most of the HEPs, and Table 20-20 presents guidelines for estimating them for the other HEPs and for adjusting the tabled UCBs for stress and type of task, e.g., dymamic rather than step-by-step, as defined in Table 20-16.

\section{Search Scineme for Use of Chapter 20 Tables}

A search scheme is presented in Figure 20-1 to aid the analyst in considering all tables of HEPs that he should consult in an HRA. This search scheme is organized according to the outline of a Technique for Human Error Prediction (THERP) procedure for HRA, as presented in Figure 5-6 and discussed in Chapter 5. The heavy lines in the search scheme represent the paths of HRA activities we have most often employed in HRAs of NPP operations. Ordinarily, the analyst will have completed an initial task analysis and a set of first-cut HRA event trees before using the search scheme. He is now ready to assign HEPs to the failure limbs in the trees. The search scheme uses the flowchart format to guide the analyst through the essential steps in the conduct of an HRA, indicating the appropriate tables to which to refer at each stage of the analysis. It is assumed that if the 

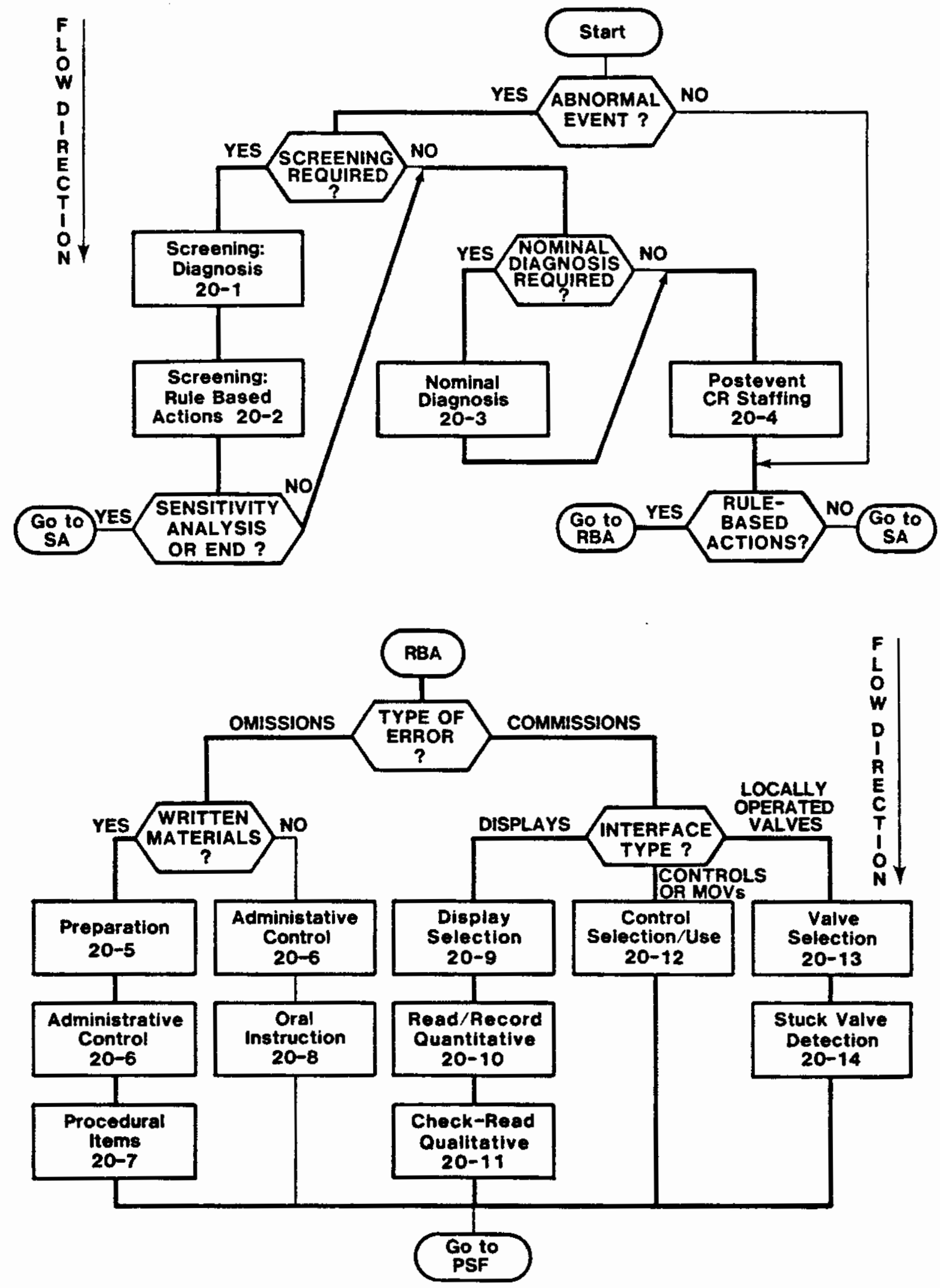

Figure 20-1 Search scheme for use of Chapter 20 tables ( 1 of 3 ). 


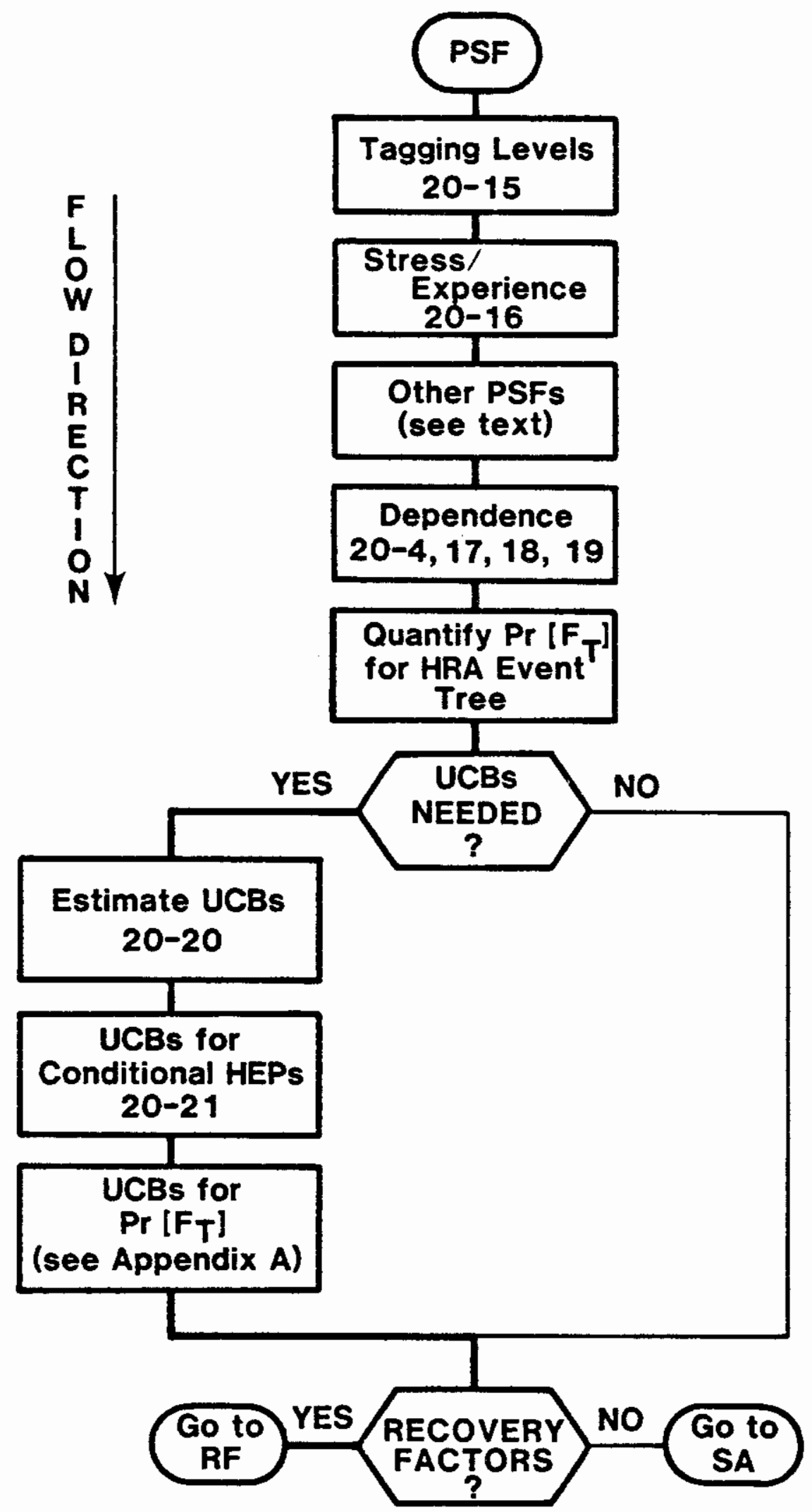

Figure 20-1 Search scheme for use of Chapter 20 tables (p 2 of 3 ). 


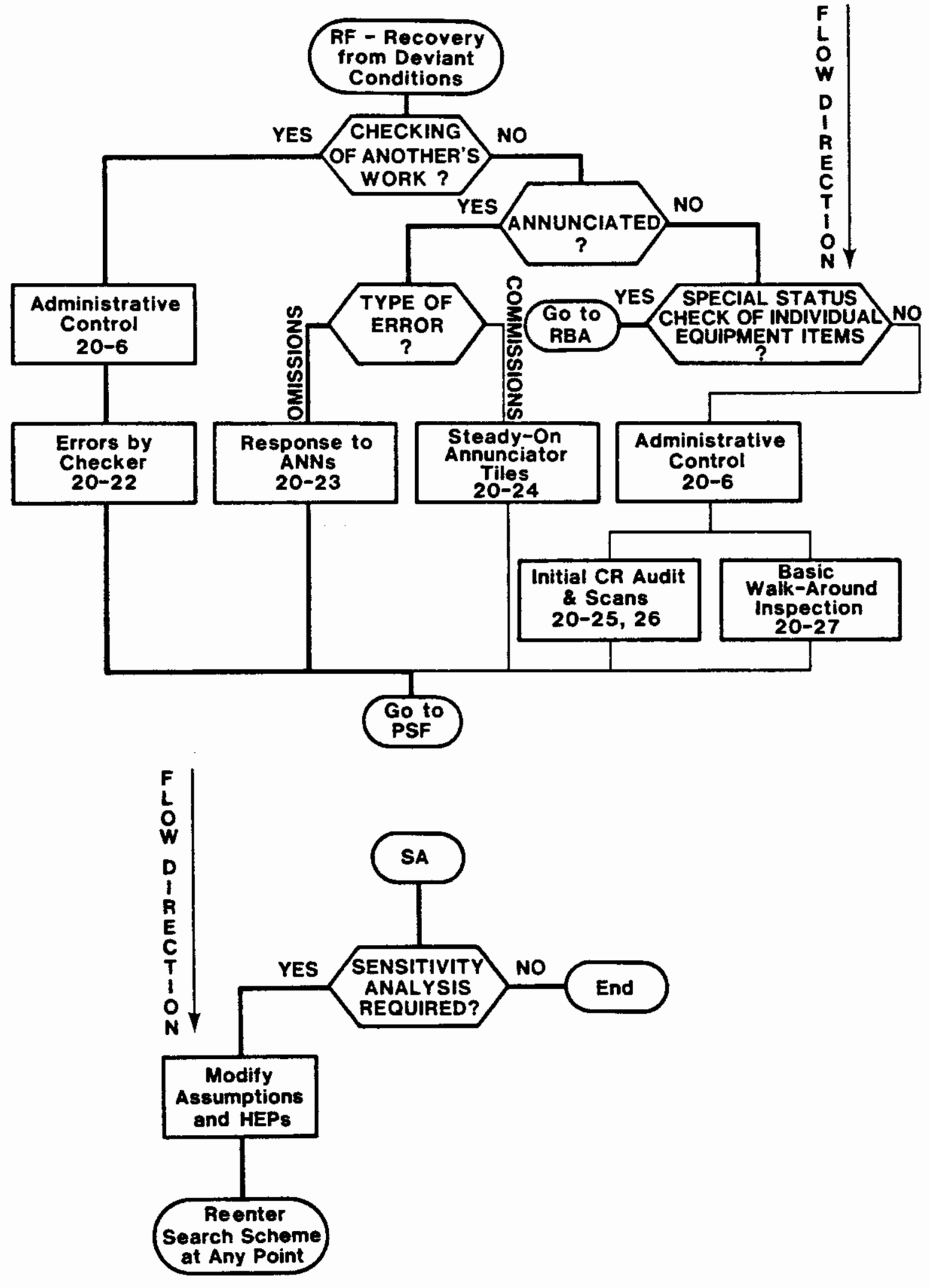

Figure 20-1 Search scheme for use of Chapter 20 tables ( $p_{3}$ of 3 ). 
analyst is directed to the appropriate table, he can select the item in the table that most closely approximates the task and conditions being evaluated. However, any tabled HEP may have to be modified according to plantspecific PSFs.

If the table to which the analyst is directed does not list an item that closely approximates the analysis task, he may select an item from some other table that matches the underlying behavioral processes identified in the task analysis. Alternatively, he may rely on judgment or seek other data sources. Some guidance is presented later, in the section entitled, "The Data Tables."

Figure 20-1 is presented here and also at the end of this chapter for the convenience of the analyst.

\section{A Talk-Through of the Search Scheme}

The search scheme in Figure 20-1 represents an iterative process, and the analyst may enter the figure at any point in the logic. The ellipses represent reference points, the hexagons represent decision nodes, and the rectangles represent action items.

To illustrate the use of the search scheme, we will enter at the "Start" ellipse and proceed through a hypothetical, complete HRA of the type described in NUREG/CR-2254. Every table will be considered in the following sequence. This talk-through is, of course, generic. To illustrate application of the search scheme for a specific sample HRA, see the first example problem in Chapter 21.

(1) ABNORMAI EVENT? This is the first decision node after "Start." Generally, the abnormal events of major interest in a HRA for a PRA are loss-of-coolant accidents (LOCAs) and transients. If addressing a LOCA or transient, follow the YES path.

(2) SCREENING REQUIRED? As described in Chapter 5, this is the next decision node on the YES path. Screening involves the assignment of very high failure probabilities to each human task. If the very high HEPs do not have a material effect on the system analysis, the task(s) may be dropped from further consideration. The decision as to whether screening is required will be made in conjunction with the system analysts. Assume YES.

(3) Screening values may be obtained for diagnostic performance and for subsequent rule-based actions (RBAs), using Tables 20-1 and 20-2.

(4) SENSITIVITY ANALYSIS OR END? For some purposes, the analysis will end with a screening analysis, or it may be followed by a sensitivity analysis (SA). For either of these cases, follow the YES path. The "Go to SA" ellipse transfers the analyst to the bottow of page 3 of the figure, where he may perform a sensitivity analysis or exit from the flowchart. If postscreening HRA is required, follow the No path. Assume No. 
(5) NOMINAI DIAGNOSIS REQUIRED? The nominal model for diagnostic performance lists HEPs that are more realistic than the HEPs in the screening model. In most PRAs, the nominal HEPs for diagnostic performance are of interest. Assume YES.

(6) The HEPs for the nominal diagnosis model are listed in Table 20-3 and are used to estimate the probability of control room (CR) personnel failing to properly diagnose one or more abnormal events within the time constraints given by the system analysts.

(7) Table 20-4 lists the CR staffing assumptions as a function of time after recognition of an abnormal event. These assumptions enable the analyst to consider the effects of personnel interaction in modifying the nominal HEPs for postevent activities (e.g., rulebased actions).

(8) RULE-BASED ACTIONS? Usually, RBAs will be evaluated in an HRA. Assume YES and go to the RBA ellipse.

(9) TYPE OF ERROR? This decision node does not have a YES/NO division. The section of the flowchart branching from this decision node and reuniting at the PSF ellipse encompasses all the rule-based tasks usually addressed in an HRA. Tables 20-5 through 20-14 list the HEPs for all the rule-based tasks specified by the action rectangles in this section. The analyst will follow the appropriate path through this section for each rule-based task being evaluated. In many HRAs, all the paths will be used. We will assume that this is the case for this HRA. All the paths flowing from the TYPE OR ERROR? hexagon will be considered before going to the "PSF" ellipse to adjust the nominal HEPs for relevant PSFs. We will address errors of omission first.

(9A) WRITTEN MATERIALS? This decision node applies to whether written materials are mandated for the task. Written materials include formal procedures, ad hoc procedures, and oral instructions that are written down by the recipient as he receives them.

- If YES, Tables 20-5, 20-6, and 20-7 list the HEPs for the preparation of written materials, for the initiation of the task and for the misuse of procedures, and for the omission of procedural items when using written materials. (Note that Table 20-5 includes errors of commission as well as errors of omission, but for convenience is placed onjy in the OMISSION path from the TYPE OF ERROR? hexagon.)

- If NO, the worker is relying on memory. Table 20-6 provides the HEPs for initiation of the task and Table 20-8 the HEPs in carrying out oral instructions as a function of the number of items to be remembered.

- Returning to the TYPE OF ERROR? hexagon, we will now consider errors of commission. 
A Talk-Through of the Search scheme

(9B) INTERFACE TYPE? Displays, controls (including switches for motoroperated valves [MOVs]), and locally operated valves are the three types of man-machine interfaces studied in HRAs.

- For some frequently practiced tasks, the analyst may judge that the probabilities of errors of commission are negligible. See the fourth example in Chapter 21.

- If DISPLAYS, the following tables list the HEPs for selection of displays (20-9), for reading and recording quantitative information from displays (20-10), and for noting the general state of displays $(20-12)$.

- If CONTROLS or MOVs, Table 20-12 lists HEPs for selection and use of switches, connectors, and other manual controls.

- If LOCALIY OPERATED VALVES, Table 20-13 lists HEPs for selecting these valves, and Table 20-14 lists HEPs for recognizing that a valve is not fully open or closed because it sticks.

(10) Transfer to the "PSF" ellipse on page 2 of Figure 20-1. These rectangles list the PSFs that should be considered when evaluating the HEPs for RBAs. The nominal HEPs in any table may not accurately represent a plant-specific situation. Depending on the quality of PSFs observed, the nominal HEP may be raised or lowered by the analyst.

(10A) Table 20-15 indicates the modifiers to be applied to HEPs for changing or restoring the normal states of safety-related components as a function of the tagging level in use. No modification of HEPs is required if the plant uses the usual level 2 tagging system.

(10B) Table 20-16 lists modifiers to be applied to HEPs for different stress levels under which a task is to be performed, according to the experience level of the personnel on duty. If a task will be performed under different levels of stress at different times, or if different experience levels of personnel will be on duty at different times, the HRA event trees must represent such fractionation, as described in Chapter 5.

(10C) The "Other PSFs" rectangle is a reminder to consider the many other PSFs mentioned in the Handbook that are not listed in the tables. In addition, almost always there are plant-specific PSFs that the analyst will observe in the course of his site visits, which should be included at this point, using judgment to estimate their effects.

(IOD) Tables 20-17, 20-18, and 20-19 present equations and tabled HEPs to be applied to the nominal HEPs to allow for the effects of different levels of dependence that may be assessed between tasks performed by one person or for the effects of dependence between people working jointly. (Table 20-4 provides initial estimates of dependence among $\mathrm{CR}$ personnel in carrying out procedures after an abnormal event.) 
(11) At this stage, the analyst following the HRA sequence shown in Figure 5-6 is ready to perform his first cut at quantifying the total-failure term, $P x\left[F_{T}\right]$, for each HRA event tree. It is at this point in a PRA that certain human error terms may be dropped from further consideration if, as determined by the system analysts, they have no material impact on the system failure events of interest.

(12) UCBE NEEDED? If point estimates of HEPs without any UCBs are adequate, follow the No path. Usually, the YES path will be followed:

- Table 20-20 provides guidelines for assigning UCBs (or EFs) to individual HEPs in the analysis. The upper and lower UCBs may be used as one form of SA, as described in Chapter 7.

- Table 20-21 provides UCBs for conditional HEPs based on use of the dependence model.

- Appendix A presents the methodology for propagation of UCBs through an HRA event tree so that UCBs may be assigned to the total-failure term, $\operatorname{Pr}\left[F_{T}\right]$, for each HRA event tree. This term plus its UCBs constitute the usual input to the system analyst for inclusion in the overall PRA.

(13) RECOVERY FACTORS? Usually recovery factors (RF) will be considered at this point in the HRA. Assume YES. Transfer to the top of page 3 of the search scheme to the "Recovery from Deviant Conditions" ellipse.

(14) CHECKING of ANOTHER'S WORK? The recovery factor from any deviant condition under normal operating conditions may depend on the direct checking of someone's work (the YES path) or on inspections of plant indications of deviant conditions. In an HRA, both paths are generally followed. We will begin with the YES path.

(15) The YES path leads to Table 20-6, which provides HEPs for the initiation of the task of the checker, and to Table 20-22, which lists HEPs for errors of omission and comission in the checker's task.

(16) The No path leads to the ANNUNCIATED? hexagon. The recovery cues may be annunciated or unannunciated. We will address both modes.

(16A) If YES, the decision node, TYPE OF ERROR?, leads to one of two tables:

- Table 20-23 presents the Annunciator Response Model listing the HEPs for an operator to initiate intended corrective action to one or more annunciators.

- Table 20-24 lists HEPs for remembering to respond to a steady-on annunciator tile after an interruption or for noticing an important steady-on annunciator tile during the initial audit or subsequent hourly scans. 
(16B) If NO, proceed to the decision node, SPECIAL STATUS CHECK OF INDIVIDUAL EQUIPMENT ITEMS? If certain displays are read according to a schedule, or if the operator is otherwise directed to read some display, follow the YES path to the "RBA" ellipse on page 1 of the flowchart. If there is no specific requirement to check the status of individual equipment items, that is, the checking is more of a general inspection, the No path leads to four tables:

- Table 20-6 lists the HEP for initiation of a scheduled checking or inspection function.

- Table 20-25 lists HEPs for detecting deviant unannunciated indications on different types of displays during the initial audit and on subsequent hourly scans.

- Table 20-26 modifies the HEPs from Table 20-25 when more than one (up to 5 ) displays are presenting deviant indications.

- Table 20-27 lists HEPs for failure of the basic walk-around inspection to detect unannunciated deviant indications of equipment within 30 days.

(17) At this point, having considered all important recovery factors, the analyst will proceed to the "PSF" ellipse to consider modifications of the recovery HEPs by relevant PSFs. After the PSFs have been considered, follow the NO path from the RECOVERY FACTORS? decision node at the bottom of page 1 of the flowchart and proceed to the "SA" ellipse on page 3 .

(18) SENSITIVITY ANALYSIS REQUIRED? The last thing done in a complete HRA is an SA, although it may be done at other times in the URA also. The $S A$ is important since it provides a means of ascertaining whether different assumptions or estimates result in materially different effects in the overall PRA. Assume YES.

(18A) As indicated in the rectangle, the analyst may use SA to modify any assumptions or HEPs, following the procedure described in Chapters 5 and 7. He may then reenter the search scheme at any point to assess changes resulting from these modifications. Reentry will take him back to the "PSF" ellipse on page 2 of the flowchart and to the recalculation of the end-failure term, $\operatorname{Pr}\left[F_{T}\right]$, using new values.

(18B) The search scheme will always take the analyst back to the SENSITIVITY ANALYSIS REQUIRED? decision node on page 3 of the flowchart. When sufficient SA has been accomplished for purposes of the PRA, the NO path from this decision node leads to the "END" ellipse, signifying the completion of the HRA.

\section{List of Chapter 20 Data Tables}

The data tables from Part III that are repeated in this chapter are listed below. Note that at the end of the title of each table, there appears in 
parentheses the table number in Part III to which the Chapter 20 table corresponds. This reference to Part III table numbers will enable the reader to quickly find background discussion of PSFs that does not appear in Chapter 20. For users familiar with the draft Handbook, Table $F-2$ in Appendix $F$ provides a cross-index of the table numbers in the revised Chapter 20 with the table numbers from the same chapter in the draft Handbook (Swain and Guttmann, 1980).

Ch. 20

Table No. Title of Table

20-1 Initial-screening model of estimated HEPs and EFs for diagnosis within time $T$ by control room personnel of abnormal events annunciated closely in time (from Table 12-2)

20-2 Initial-screening model of estimated HEPs and EFs for rulebased actions by control room personnel after diagnosis of an abnormal event (from Table 12-3)

20-3 Nominal model of estimated HEPs and EFs for diagnosis within time $T$ by control room personnel of abnormal events annunciated closely in time (from Table 12-4)

20-4 Number of reactor operators and advisors available to cope with an abnormal event and their related levels of dependence: assumptions for PRA (from Table 18-2)

20-5 Estimated HEP per item (or perceptual unit) in preparation of written material (from Tabie 15-2)

20-6 Estimated HEPs related to failure of administrative control (from Table 16-1)

20-7 Estimated probabilities of errors of amission per item of instruction when use of written procedures is specified (from Table 15-3)

20-8 Estimated probabilities of errors in recalling oral instruction items not written down (from Table 15-1)

20-9 Estimated probabilities of errors in selecting unannunciated displays for quantitative or qualitative readings (from Table $(1-2)$

20-10 Estimated HEPs for errors of commission in reading and recording quantitative information from unannunciated displays (from Table $(1-3)$

20-11 Estimated HEPs for errors of commission in checking-reading displays (from Table 11-4) 
Ch. 20

Table No.

Title of Table

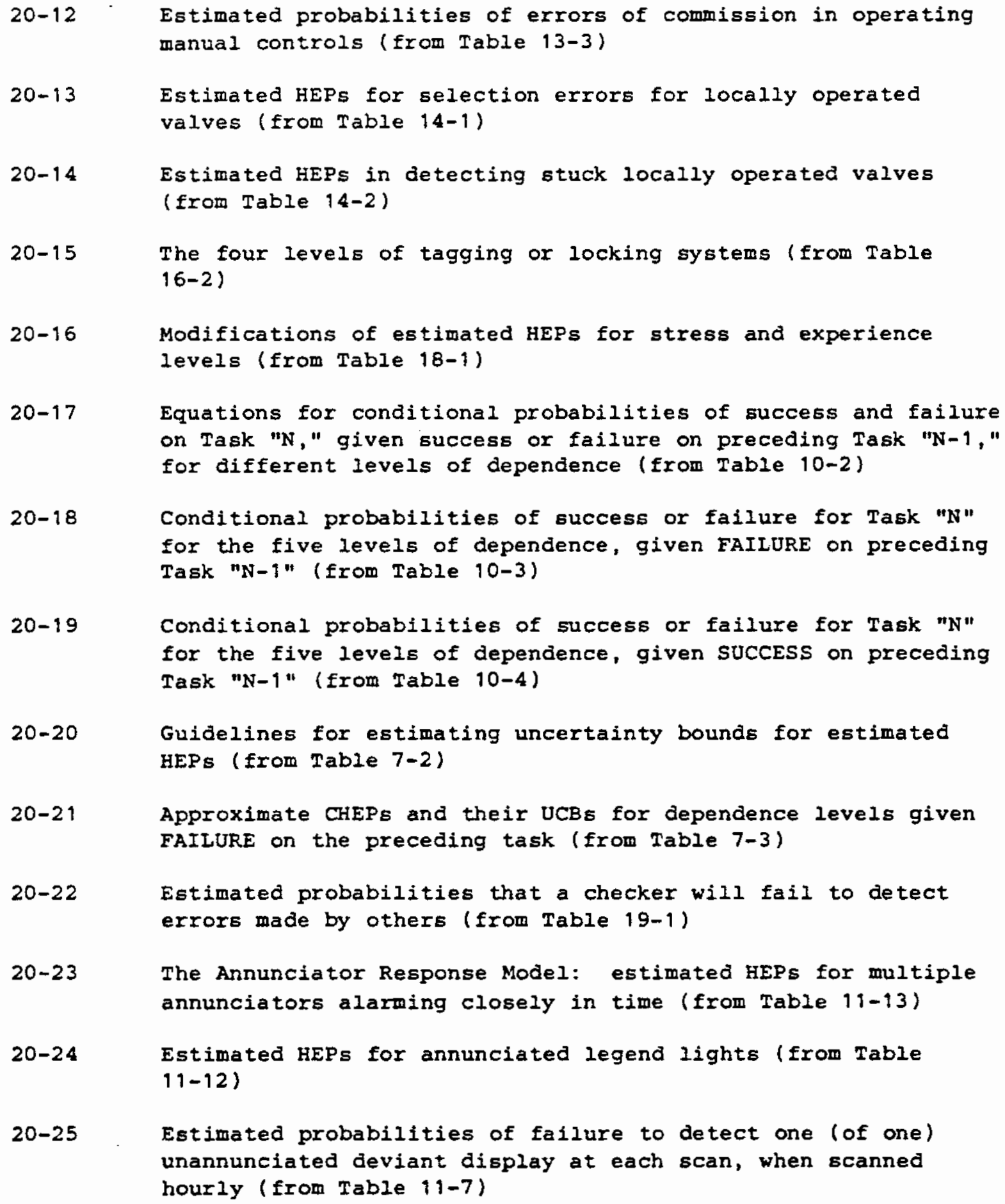


Ch. 20

Table No.

$20-26$

$20-27$ mitle of Table

\begin{abstract}
Estimated probabilities of failing to detect at least one of one to five unannunciated deviant displays as a function of the BHEP for detection of a single deviant display during periodic scanning (from Table 11-6)

Estimated probabilities that the basic walk-around inspection will fail to detect a particular deviant indication of equipment outside the control room within 30 days (from Table 19-4)
\end{abstract}

This section presents the 27 data tables extracted from Part III. TO facilitate rapid access to these tables, a table designator for each table is shown in large print in the outer upper corner of the page on which the table appears. The table designators are expressed without the chapter prefix (e.g., Table 20-6 is expressed as 6).

Figure 20-2, which precedes the first table, is a quick reference guide to the tables, organized under the seven major headings that are used in the search scheme (Figure 20-1). For convenience, Figure 20-2 also appears as the last page in Chapter 20.

We remind the user that the tables in this chapter do not stand alone. They must be considered in association with the descriptive material in those chapters that include the original versions of the tables. It is not possible to include all of the relevant PSFs in each table; the complete Handbook must be used.

Obviously, the tables cannot list every act or task that could take place in an NPP--only the most frequently observed tasks are listed. When a task is being evaluated for which we have no tabled HEPs, we assign a nominal HEP of .003 as a general error of omission or commission if we judge there is some probability of either type of error. When evaluating abnormal events, we assign a nominal HEP of .001 to those tasks for which the tables or text indicate that the HEP is "negligible" under normal conditions. The nominal HEP of .001 allows for the effects of stress that are associated with abnormal events.

Most of the tables list the EFs or UCBs for the HEPs. For cases in which the EFs or UCBs are not listed, Table 20-20 presents guidelines for estimating them. In the course of an SA, the nominal HEP for some task may change significantly as different assumptions are evaluated. Note that the EFs may change when a nominal HEP is changed; for example, under certain assumptions, some task may have a tabled HEP of, say, .008, with an EF of 3. If the assumptions are modified so that the HEP is doubled (to .016), the EF would change frow 3 to 5 (see the second and third items in Table 20-20). Also remember that stress and other PSFs may increase the EFs, as indicated in Table 20-20. 
Figure 20-2

Screening $\left[\begin{array}{l}\text { Diagnosis } 1 \\ \text { Rule-Based Actions } 2\end{array}\right.$

Diagnosis

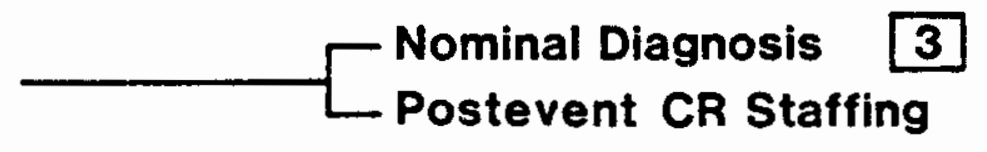

4

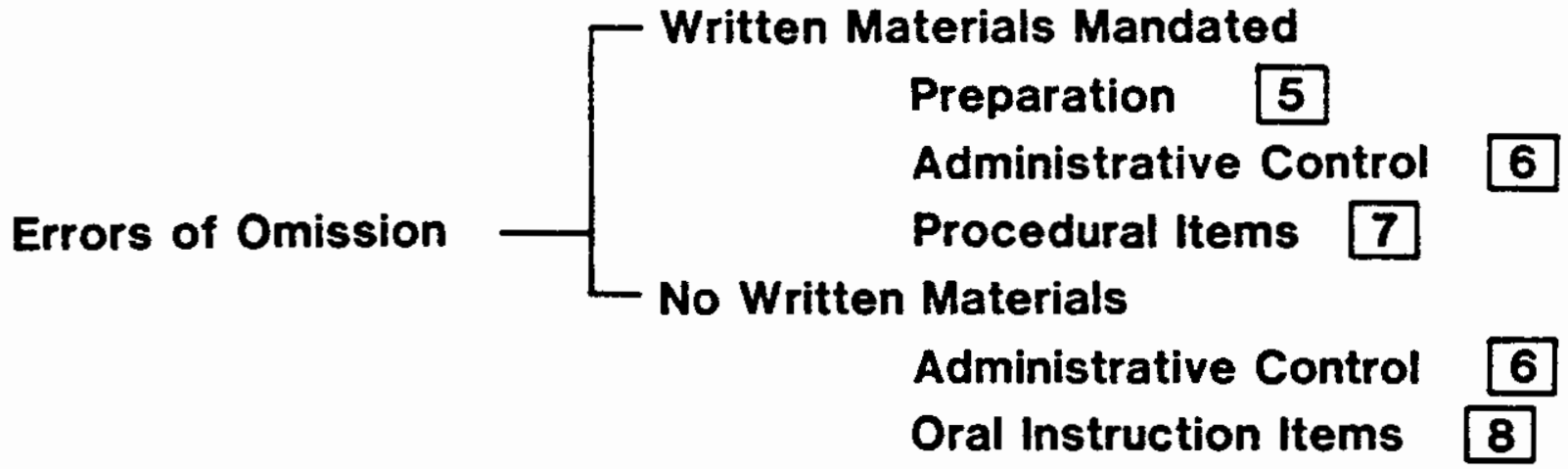

Displays Display Selection 9

Read/Record Quantitative 10

Errors of Commission Check-Read Quantitative 11

- Control \& MOV Selection \& Use 12

- Locally Operated Valves

Valve Selection 13

Stuck Valve Detection 14

PSFs $1 \begin{aligned} & \text { Tagging Levels } 15 \\ & \text { Stress/Experience } 16 \\ & \text { Dependence } 17 \\ & \text { Other PSFs (see text) }\end{aligned}$

Uncertainty Bounds - Estimate UCBs 20

Recovery Factors $-\left[\begin{array}{l}\text { Errors by Checker } \\ \text { Annunciated Cues } 22 \\ \text { Control Room Scanning } \\ \text { Basic Walk-Around Inspection }\end{array}\right.$

Figure 20-2 Quick reference guide to Chapter 20 tables. 
For record-keeping convenience in an HRA, the left-most coluun for most of the tables is headed by the word, "Item." In keeping a record of which tabled entries are used in an HRA, reference can be made to a particular table and item number, e.g., T20-1, \#1. In some of the tables, e.g., Table 20-8, it is convenient to use small letters to designate separate columns of estimated HEPs. For example, in Table 20-8, Item 1 a refers to the HEP of $.001(E F=3)$, which is the top listing in the first column of HEPs. Record keeping for an HRA is illustrated in the first case study in Chapter 21 . 
THIS PAGE INTENTIONALLY LEFT BLANK 
Table 20-1 Initial-screening model of estimated HEPs and EFS for diagnosis within time $T$ by control room personnel of abnormal events annunciated closely in time (from Table 12-2)

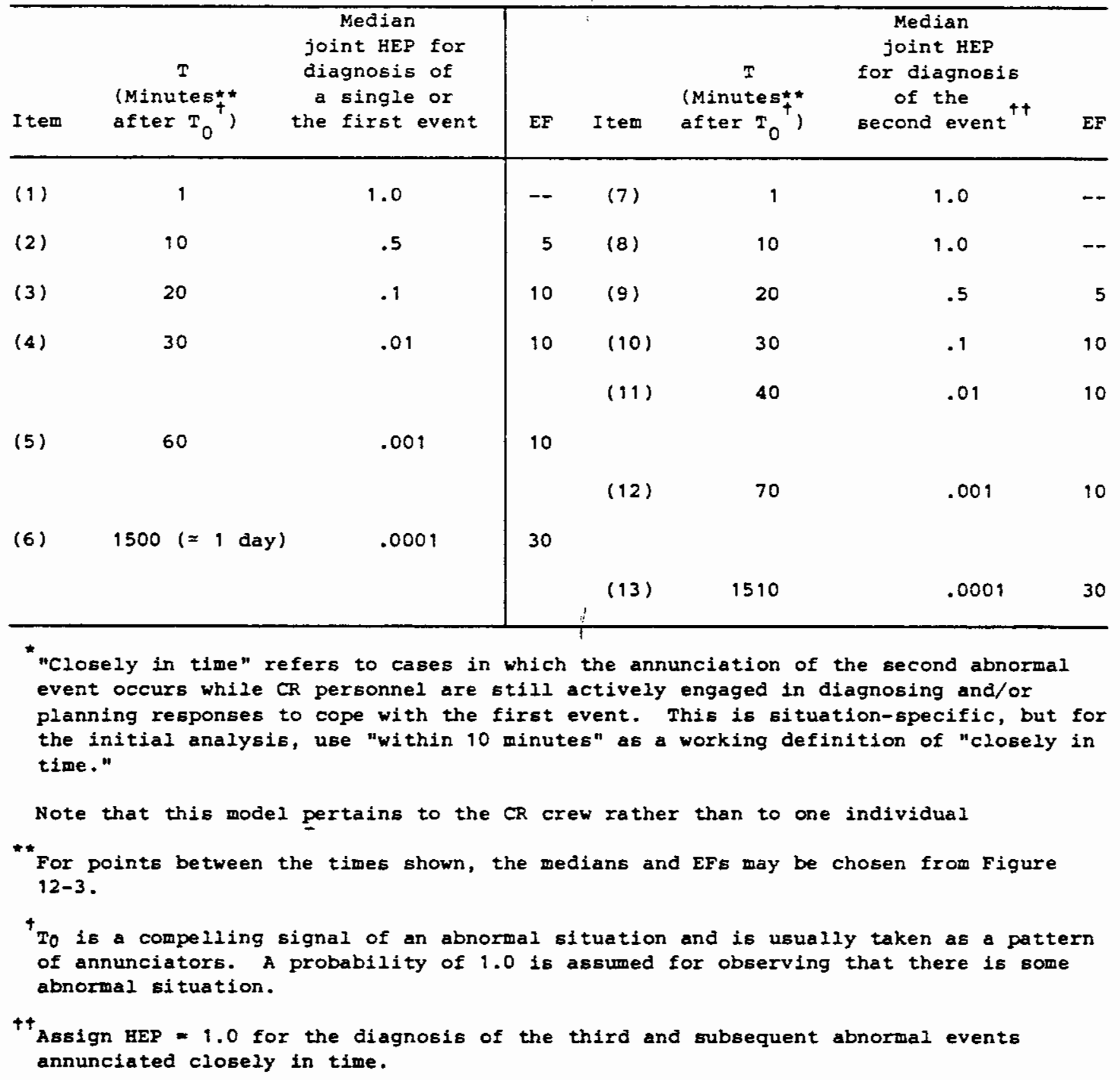


Table 20-2 Initial-screening model of estimated HEPs and EFS for rule-based actions by control room personnel after diagnosis of an abnormal event* (from Table 12-3)

\begin{tabular}{lllll}
\hline Item & Potential Errors & HEP & EF \\
\hline
\end{tabular}

Failure to perform ruze-based actions correctly when written procedures are available and used:

(1)

(2)

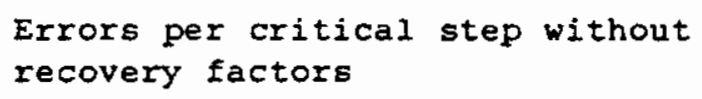

Failure to perform rule-based actions correctly when written procedures are not available or used:

$$
\begin{aligned}
& \text { Errors per critical step with or } \\
& \text { without recovery factors }
\end{aligned}
$$

1.0

*

Note that this model pertains to the $\mathrm{CR}$ crew rather than to one individual. 


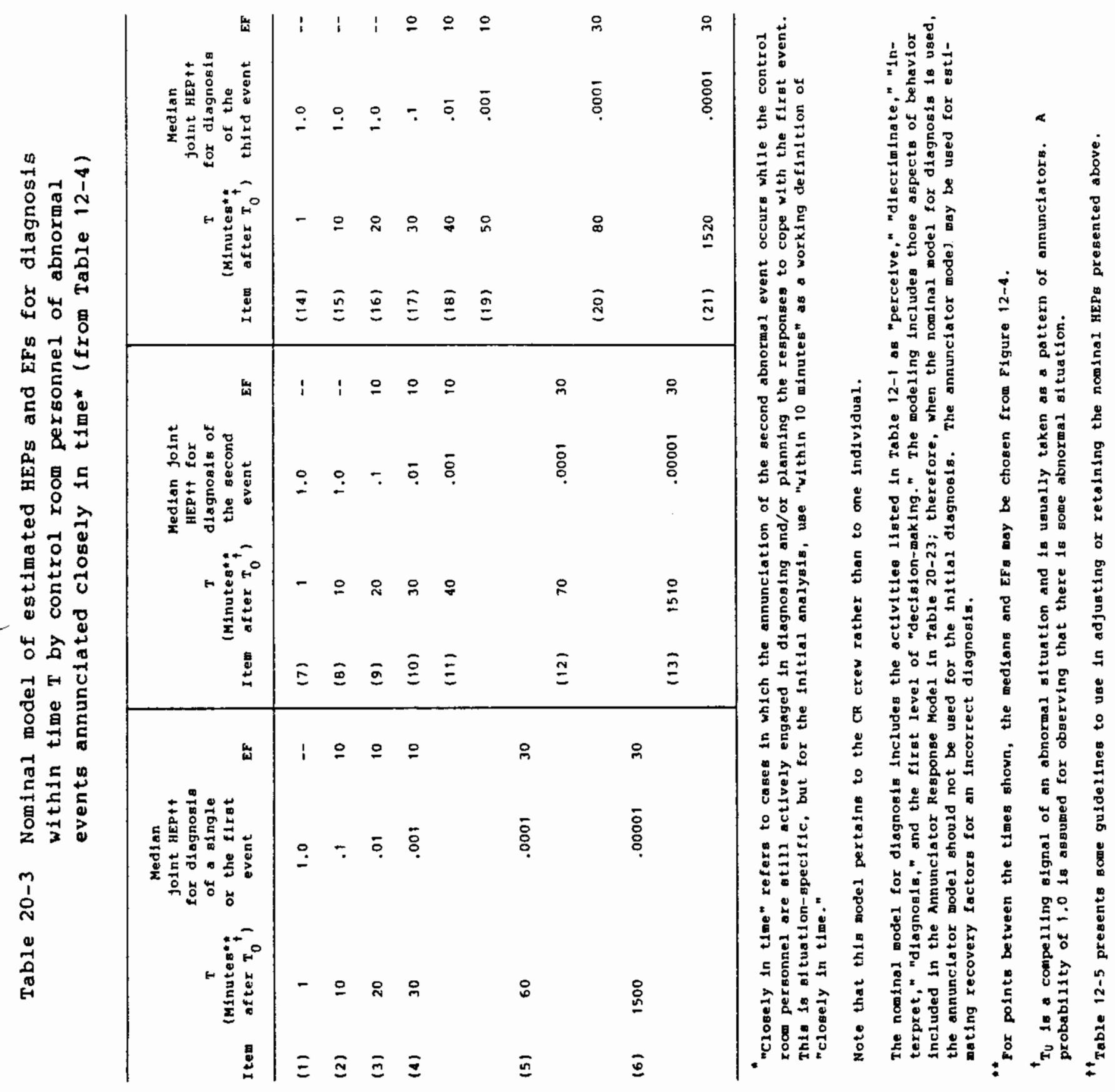


Table 20-4 Number of reactor operators and advisors available to cope with an abnormal event and their related leveis of dependence: assumptions for PRA* (from Table 18-2)

\section{Time after recognition** of an abnormal event}

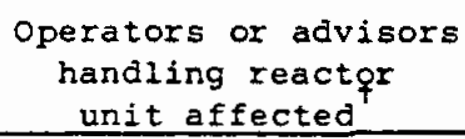

\section{Dependence levels \\ with others ${ }^{+t}$}

Item

(1)

(2)

(3)

(4)
0 to 1 minute

at 1 minute

at 5 minutes

at 15 minutes

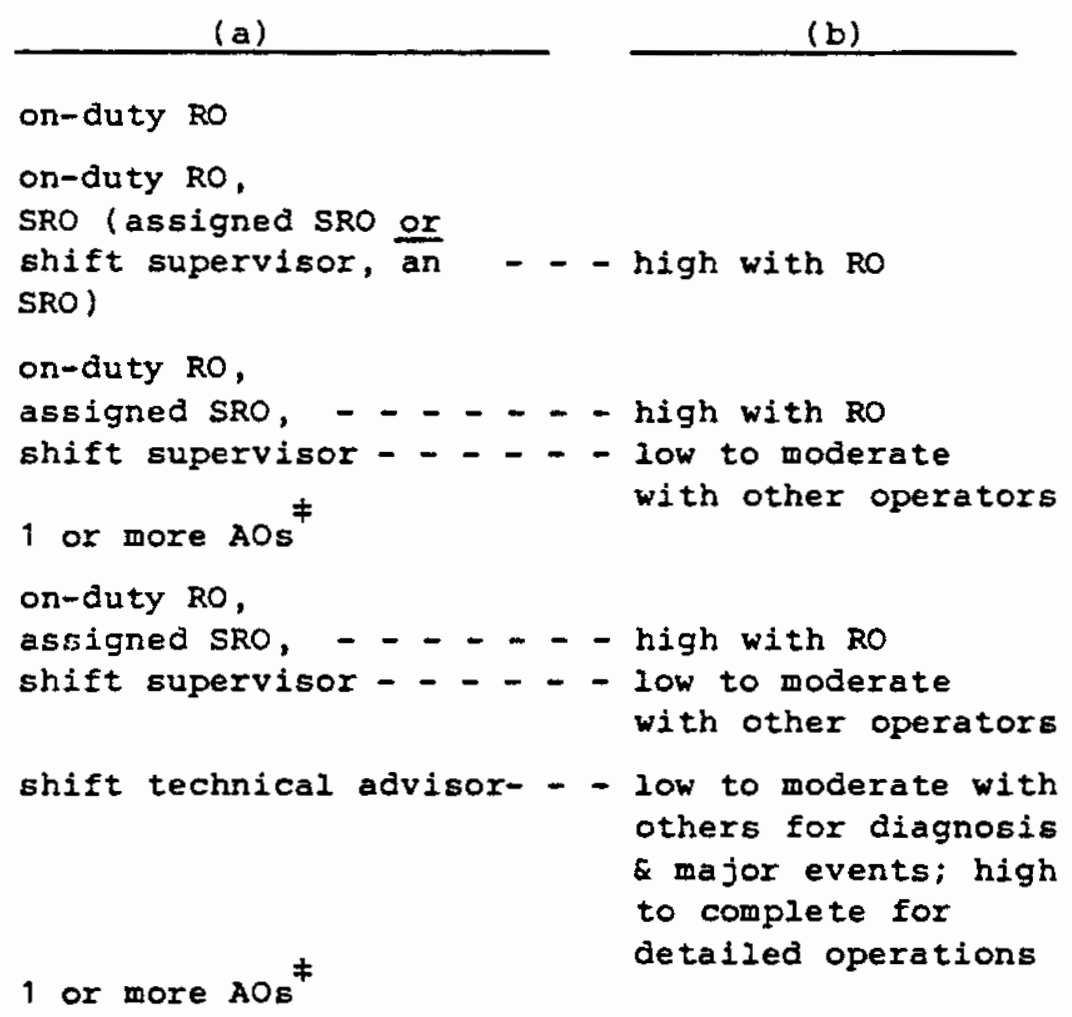

(b)

on-duty Ro, SRO (assigned SRO or shift supervisor, an - - high with RO SRO)

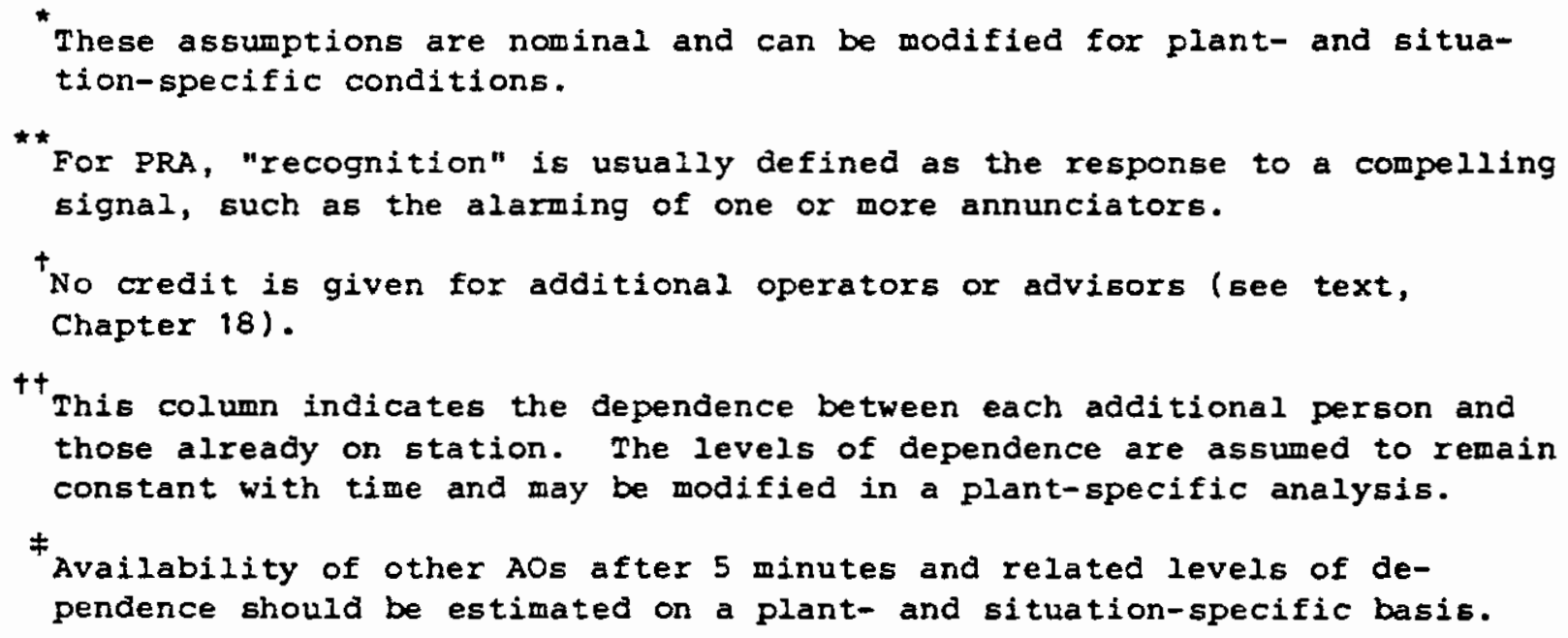


Table 20-5 Estimated HEP per item (or perceptual unit) in preparation of written material* (from Table 15-2)

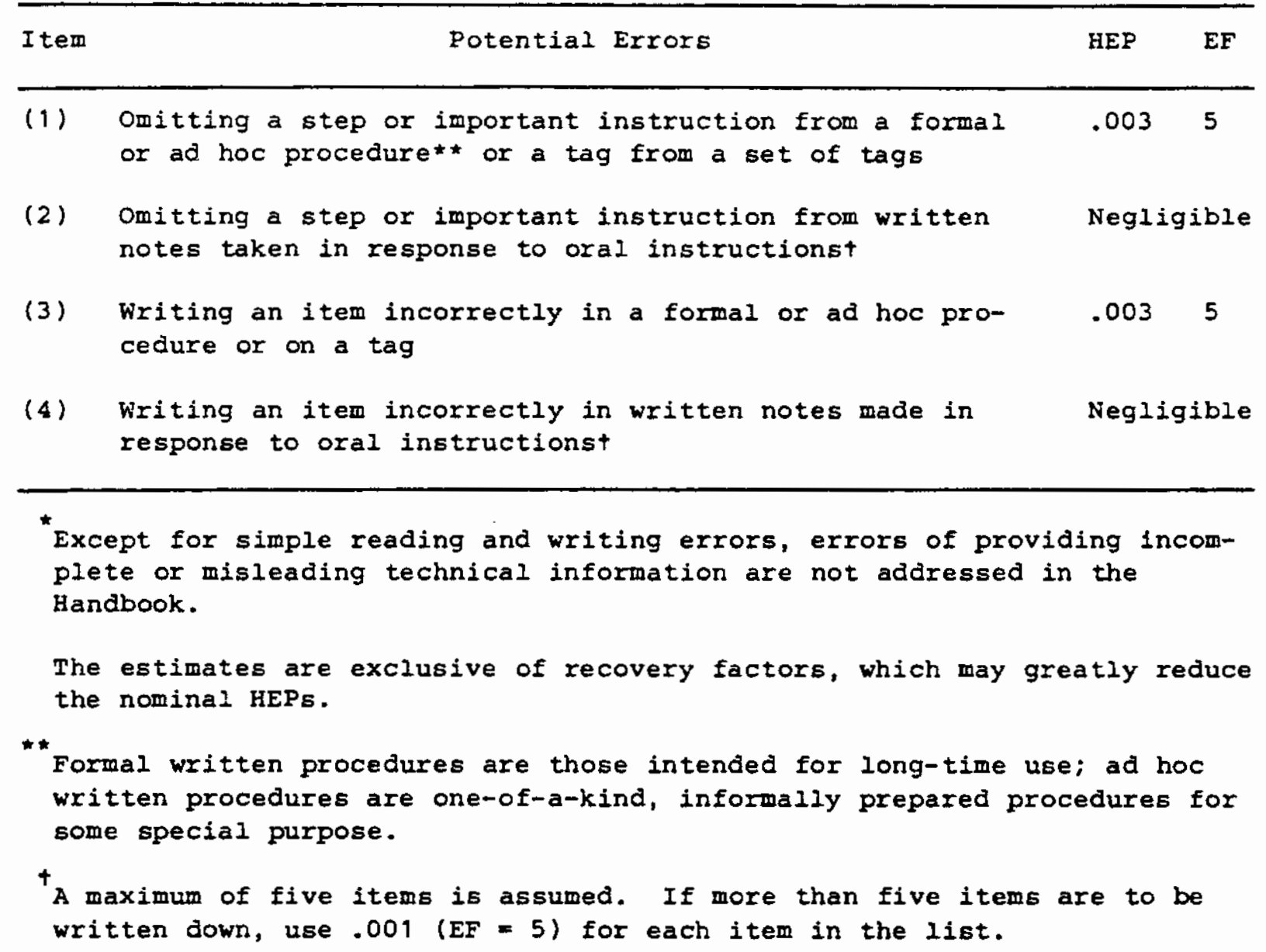


Table 20-6 Estimated HEPs relatea to failure of

administrative control (from Table 16-1)

\begin{tabular}{llll}
\hline Item & \multicolumn{1}{c}{ Task } & HEP & EF \\
\hline (1) & $\begin{array}{l}\text { Carry out a plant policy or scheduled tasks } \\
\text { such as periodic tests or maintenance per- } \\
\text { formed weekly, monthly, or at longer intervals }\end{array}$ & .01 & 5 \\
& $\begin{array}{l}\text { Initiate a scheduled shiftly checking or } \\
\text { inspection function* }\end{array}$ & .001 & 3 \\
(2) & Use written operations procedures under & & \\
& $\quad$ normal operating conditions & .01 & 3 \\
(3) & abnormal operating conditions & .005 & 10 \\
(4) & Use a valve change or restoration list & .01 & 3 \\
(5) & Use written test or calibration procedures & .05 & 5 \\
(7) & Use written maintenance procedures & .3 & 5 \\
(8) & Use a checklist properly* & .5 & 5 \\
\hline
\end{tabular}

\footnotetext{
*Assumptions for the periodicity and type of control room scans are discussed in Chapter 11 in the section, "A General Display Scanning Mode1." Assumptions for the periodicity of the basic walk-around inspection are discussed in Chapter 19 in the section, "Basic WalkAround Inspection."

$\star \star$

Read a single item, perform the task, check off the item on the list. For any item in which a display reading or other entry must be written, assume correct use of the checklist for that item.
} 
THERP

Table 20-7 Estimated probabilities of errors of omission per item of instruction when use of written procedures is specified* (from Table 15-3)

Item*

Omission of item:

HEP

EF

When procedures with checkoff, provisions are correctly used ${ }^{\dagger}$ :

(1)

$$
\text { Short list, }<10 \text { items }
$$$$
.0093
$$

$$
\text { Long list, }>10 \text { items }
$$

When procedures without checkoff provisions are used, or when checkoff provisions are incorrectly used ${ }^{t+}$ :

$$
\text { Short 1ist, } \leqslant 10 \text { items }
$$$$
.003 \quad 3
$$$$
\text { Long list, }>10 \text { items }
$$$$
.013
$$

$$
\begin{aligned}
& \text { When written procedures are avail- } \\
& \text { able and should be used but are not used }
\end{aligned}
$$$$
.05^{*} \quad 5
$$

\footnotetext{
* The estimates for each item (or perceptual unit) presume zero dependence among the items (or units) and must be modified by using the dependence model when a nonzero level of dependence is assumed.

The term "item" for this column is the usual designator for tabled entries and does not refer to an item of instruction in a procedure.

tCorrect use of checkoff provisions is assumed for items in which written entries such as numerical values are required of the user.

${ }^{+t}$ Table 20-6 lists the estimated probabilities of incorrect use of checkoff provisions and of nonuse of available written procedures.

"If the task is judged to be "second nature," use the lower uncertainty bound for .05, i.e., use .01 (EF = 5).
}

** 
Table 20-8 Estimated probabilities of errors in recalling oral instruction items not written down* (from Table 15-1)

HEPs as a function of number of items to be remembered**

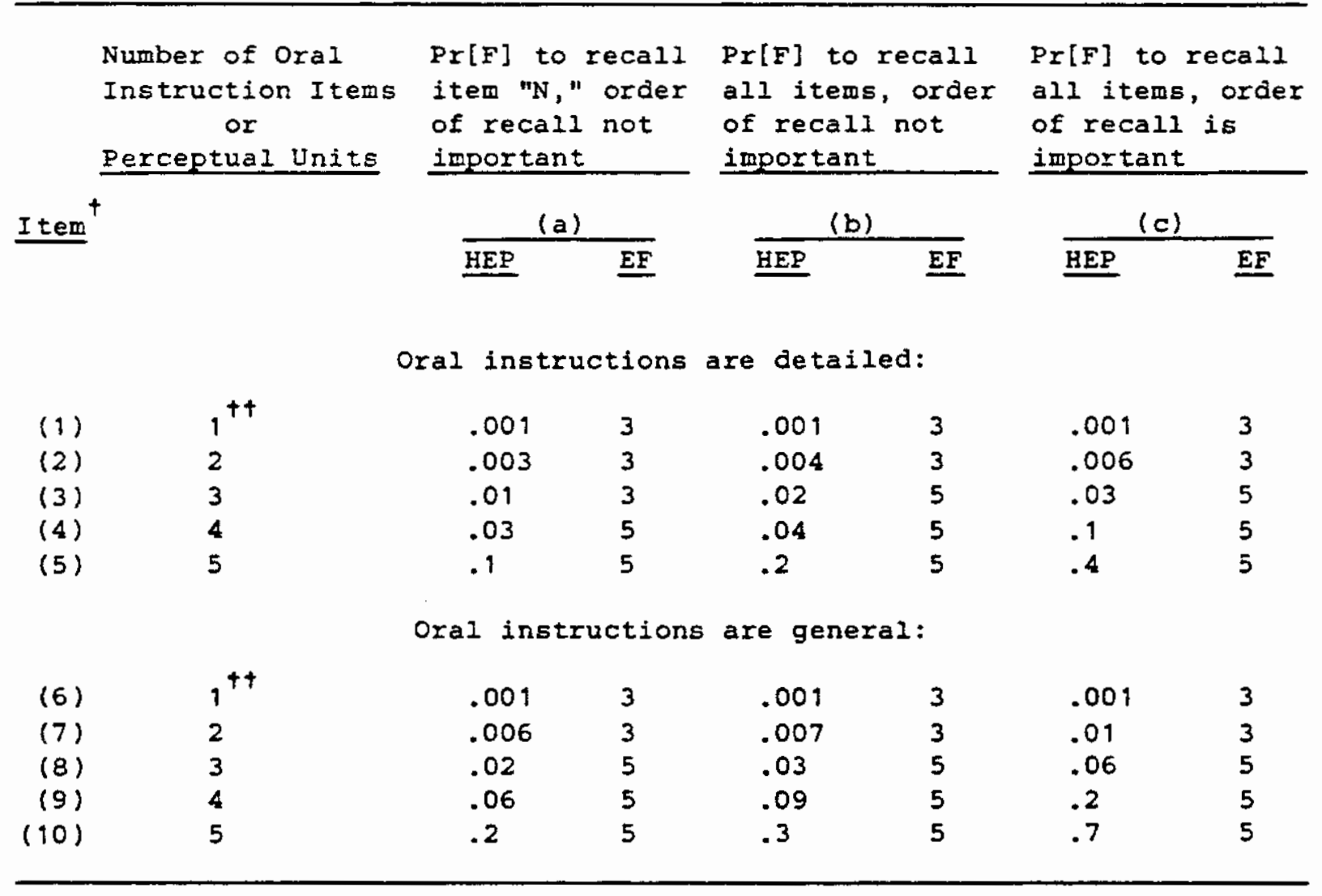

*It is assumed that if more than five oral instruction items or perceptual units are to be remembered, the recipient will write them down. If oral instructions are written down, use Table 20-5 for errors in preparation of written procedures and Table 20-7 for errors in their use.

* The first column of HEPs (a) is for individual oral instruction items, e.9., the second entry, .003 (item 2a), is the Px[F] to recall the second of two items, given that one item was recalled, and order is not important. The HEPs in the other columns for two or more oral instruction items are joint HEPs, e.g., the .004 in the second column of HEPs is the Pr[F] to recall both of two items to be remembered, when order is not important. The .006 in the third column of HEPs is the Pr[F] to recall both of two items to be remembered in the order of performance specified. For all columns, the EFs are taken from Table 20-20 as explained in Chapter 15.

the term "item" for this column is the usual designator for tabled entries and does not refer to an oral instruction item.

the Pr[F]s in rows 1 and 6 are the same as the Pr[F] to initiate the task. 
Table 20-9 Estimated probabilities of errors in selecting unannunciated displays for quantitative or qualitative readings (from Table 11-2)

(4)

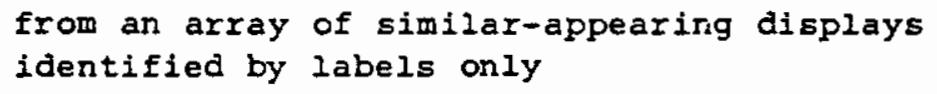

$\star$

The listed HEPs are independent of recovery factors. In some cases, the content of the quantitative or qualitative indication from an incorrect display may provide immediate feedback of the selection error, and the total exror can be assessed as negligible.

$\star \star$

"This assumes the operator knows the characteristics of the display for which he is searching. 


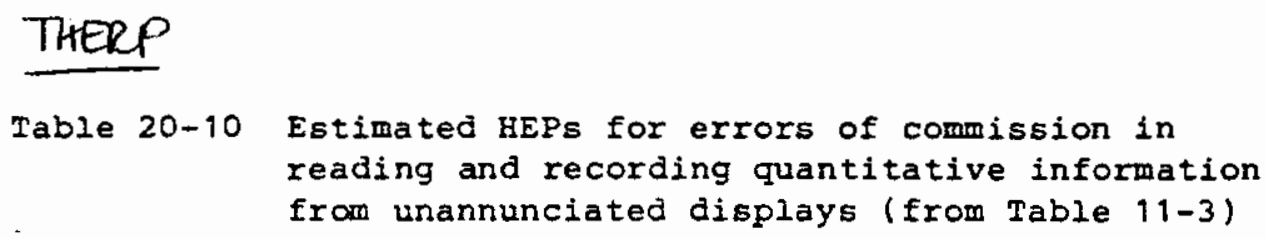

\begin{tabular}{|c|c|c|c|}
\hline I tem & Display or Task & HEP * & EF \\
\hline (1) & Analog meter & .003 & 3 \\
\hline (2) & Digital readout ( $<4$ digits) & .001 & 3 \\
\hline (3) & Chart recorder & .006 & 3 \\
\hline (4) & $\begin{array}{l}\text { Printing recorder with large } \\
\text { number of parameters }\end{array}$ & .05 & 5 \\
\hline (5) & Graphs & .01 & 3 \\
\hline (6) & $\begin{array}{l}\text { Values from indicator lamps } \\
\text { that are used as quanti- } \\
\text { tative displays }\end{array}$ & .001 & 3 \\
\hline (7) & $\begin{array}{l}\text { Recognize that an instrument } \\
\text { being read is jammed, if } \\
\text { there are no indicators } \\
\text { to alert the user }\end{array}$ & .1 & 5 \\
\hline & $\begin{array}{l}\text { Recording task: Number of } \\
\text { digits or letters* to be } \\
\text { recorded }\end{array}$ & & \\
\hline$(8)$ & $<3$ & Negligible & - \\
\hline (9) & $>3$ & $\begin{array}{l}.001 \text { (per } \\
\text { symbol) }\end{array}$ & 3 \\
\hline$(10)$ & $\begin{array}{l}\text { Simple arithmetic calcula- } \\
\text { tions with or without } \\
\text { calculators }\end{array}$ & .01 & 3 \\
\hline (11) & $\begin{array}{l}\text { Detect out-of-range } \\
\text { arithmetic calculations }\end{array}$ & .05 & 5 \\
\hline
\end{tabular}

* Multiply HEPs by 10 for reading quantitative values under a high level of stress if the design violates a strong populational stereotype; e.g., a horizontal analog meter in which values increase from right to left.

" In this case, "letters" refer to those that convey no meaning. Groups of letters such as MOV do convey meaning, and the recording HEP is considered to be negligible. 
TTHERP

Table 20-11 Estimated HEPs for errors of commission in check-reading displays* (from Table 11-4)

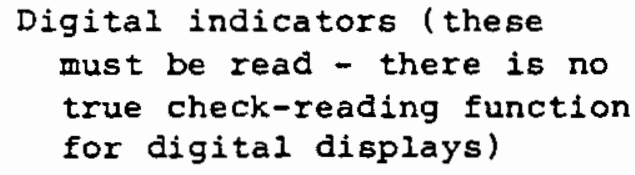
true check-reading function for digital displays)

Analog meters:

$\begin{array}{lll}\text { with easily seen limit marks } & .001 & 3 \\ \begin{array}{l}\text { with difficult-to-see limit } \\ \text { marks, such as scribe lines }\end{array} & .002 & 3 \\ \text { without limit marks } & .003 & 3\end{array}$

Analog-type chart recorders:

$\begin{array}{lll}\text { with limit marks } & .002 & 3 \\ \text { without limit marks } & .006 & 3\end{array}$

Confirming a status change Negligible** on a status lamp

Misinterpreting the indication on the indicator lamps

Negligible ${ }^{+}$

\footnotetext{
"Check-reading" means reference to a display merely to see if the indication is within allowable limits; no quantitative reading is taken. The check-reading may be done from memory or a written checklist may be used. The HEPs apply to displays that are checked individually for some specific purpose, such as a scheduled requirement, or in response to some developing situation involving that display. 
THERP

Table 20-12 Estimated probabilities of errors of commission in operating manual controls* (from Table 13-3)

\begin{tabular}{|c|c|c|c|}
\hline I tem & Potential Errors & HEP & EF \\
\hline (1) & $\begin{array}{l}\text { Inadvertent activation of a control } \\
\text { select wrong control on a panel from an array of } \\
\text { similar-appearing controls**: }\end{array}$ & see text, & Ch. 13 \\
\hline (2) & identified by labels only & .003 & 3 \\
\hline (3) & arranged in well-delineated functional groups & .001 & 3 \\
\hline (4) & $\begin{array}{l}\text { which are part of a well-defined mimfc layout } \\
\text { Turn rotary control in wrong direction (for two- } \\
\text { position switches, see ttem } 8 \text { ): }\end{array}$ & .0005 & 10 \\
\hline (5) & $\begin{array}{l}\text { when there is no violation of populational } \\
\text { stereotypes }\end{array}$ & .0005 & 10 \\
\hline (6) & $\begin{array}{l}\text { when design violates a strong populational } \\
\text { stereotype and operating conditions are } \\
\text { normal }\end{array}$ & .05 & 5 \\
\hline (7) & $\begin{array}{l}\text { when design violates a strong populational } \\
\text { stereotype and operation is under high } \\
\text { stress }\end{array}$ & .5 & 5 \\
\hline (8) & $\begin{array}{l}\text { Turn a two-position switch in wrong direction or } \\
\text { leave it in the wrong betting }\end{array}$ & + & \\
\hline (9) & $\begin{array}{l}\text { Set a rotary control to an incorrect setting } \\
\text { (for two-position switches, see item 8) }\end{array}$ & .001 & $10^{+t}$ \\
\hline$(10)$ & $\begin{array}{l}\text { Failure to complete change of state of a } \\
\text { component if switch must be held until change } \\
\text { is completed }\end{array}$ & .003 & 3 \\
\hline & $\begin{array}{l}\text { Select wrong circuit breaker in a group of } \\
\text { circuit breakers*t: }\end{array}$ & & \\
\hline (11) & densely grouped and identified by labels only & .005 & 3 \\
\hline$(12)$ & $\begin{array}{l}\text { in which the PSFs are more favorable } \\
\text { (see Ch. 13) }\end{array}$ & .003 & 3 \\
\hline (13) & $\begin{array}{l}\text { Improperly mate a connector (this includes } \\
\text { fallures to seat connectors completely and } \\
\text { fallure to test locking features of connectors } \\
\text { for engagement) }\end{array}$ & .003 & 3 \\
\hline
\end{tabular}

\footnotetext{
The HEPs axe for errors of comission only and do not include any errors of decision as to which controls to activate.

- If controls or circuit breakers are to be restored and are tagged, adjust the tabled HEPs according to rable 20-15.

tDivide HEPs for rotary controls (1tems 5-7) by 5 (use same EFs). $++$

This error is a function of the clarity with which indicator position can be determined: designs of control knobs and thetr position indications vary greatly. For plant-specific anelyses, an EF of 3 may be used.
} 
THERP

Table 20-13 Estimated HEPs for selection errors for locally operated valves (from Table 14-1)

Item Potential Errors

HEP EF

Making an error of selection in changing or restoring a locally operated valve when the valve to be manipulated is

(1) Clearly and unambiguously labeled, set apart .001 3 from valves that are similar in all of the following: size and shape, state, and presence of tags*

(2) Clearly and unambiguously labeled, part of $.003 \quad 3$ a group of two or more valves that are similar in one of the following: size and shape, state, or presence of tags*

(3) Unclearly or ambiguously labeled, set apart .005 3 from valves that are similar in all of the following: size and shape, state, and presence of tags*

(4) Unclearly or ambiguously labeled, part of a .0083 group of two or more valves that are similar in one of the following: size and shape, state, or presence of tags*

(5) Unclearly or ambiguously labeled, part of a $.01 \quad 3$ group of two or more valves that are similar in all of the following: size and shape, state, and presence of tags*

*Unless otherwise specified, Level 2 tagging is presumed. If other levels of tagging are assessed, adjust the tabled HEPs according to Table 20-15. 


\section{THERP}

Table 20-14 Estimated HEPs in detecting stuck locally operated valves (from Table 14-2)

Given that a locally operated valve sticks as it is being changed or restored, the operator fails to notice the sticking valve, when it has

A position indicator* only .0013

A position indicator* and a rising stem .0023

A rising stem but no position indicator** $.005 \quad 3$

\footnotetext{
*Equipment reliability specialists have estimated that the probability of a valve's sticking in this manner is approximately .001 per manipulation, with an error factor of 10 . position of the valve relative to a fully opened or fully closed position. A rising stem qualifies as a position indicator if there is a scale associated with it.
} 
Modifications

to Nominal

Level

Description

HEPS *

1 A specific number of tags is issued for each job Each tag is numbered or otherwise uniquely identified. A record is kept of each tag, and a record of each tag issued is entered in a suspense sheet that indicates the expected time of return of the tag; this suspense sheet is checked each shift by the shift supervisor. An operator is assigned the job of tagging controller as a primary duty. For restoram tion, the numbers on the removed tags are checked against the item numbers in the records, as a recovery factor for errors of omission or selection. OR The number of keys is carefully restricted and under direct control of the shift supervisor. A signout board is used for the keys. Keys in use are tagged out, and each incoming shift supervisor takes an inventory of the keys.

2 Tags are not accounted for individually--the operator

Use nominal HEPs may take an unspecified number and use them as required. In such a case, the number of tags in his possession does not provide any cues as to the number of items remaining to be tagged. For restoration, the record keeping does not provide a thorough checking for errors of omission or selection. If an operator is assigned as tagging controller, it is a collateral duty, or the position is rotated among operators too frequently for them to maintain adequate control tags and records and to retain skill in detecting errors of omission or selection. OR The shift supervisor retains control of the keys and records their issuance but does not use visual aids such as signout boards or tags.

3 Tags are used, but record keeping is inadequate to Use upper UCBs provide the shift supervisor with positive knowledge of every item of equipment that should be tagged or restored. No tagging controller is assigned. OR Keys are generally available to users without logging requirements.

4 No tagging system exists. OR

Perform separate No locks and keys are used. analysis

\footnotetext{
"The nominal HEPs are those in the Handbook that relate to tasks involving the application and removal of tags and, unless otherwise specified, are based on Level 2 tagging.
} 


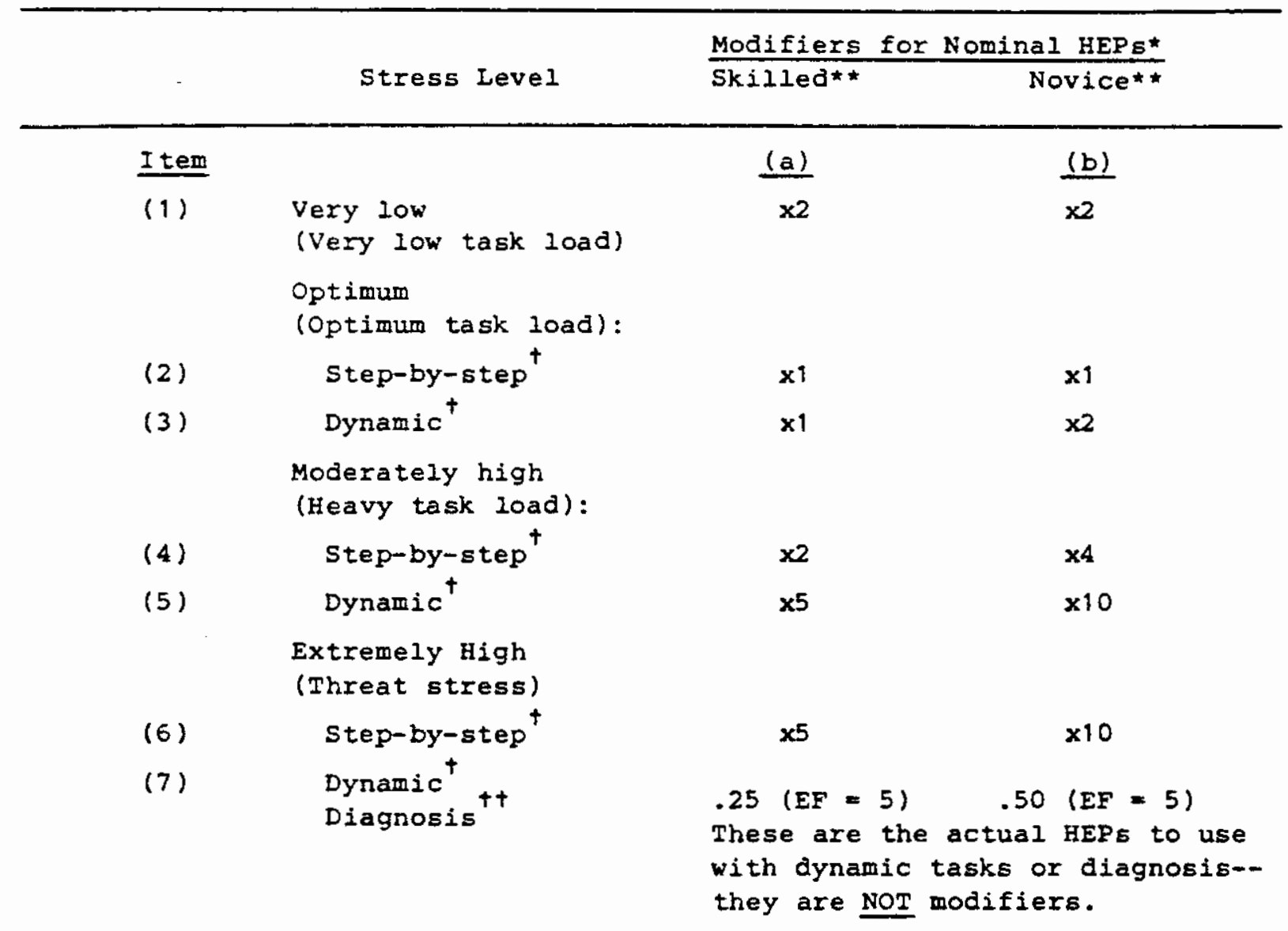

\footnotetext{
* The nominal HEPs are those in the data tables in Part III and in Chapter 20. Error factors (EFs) are listed in Table 20-20. 


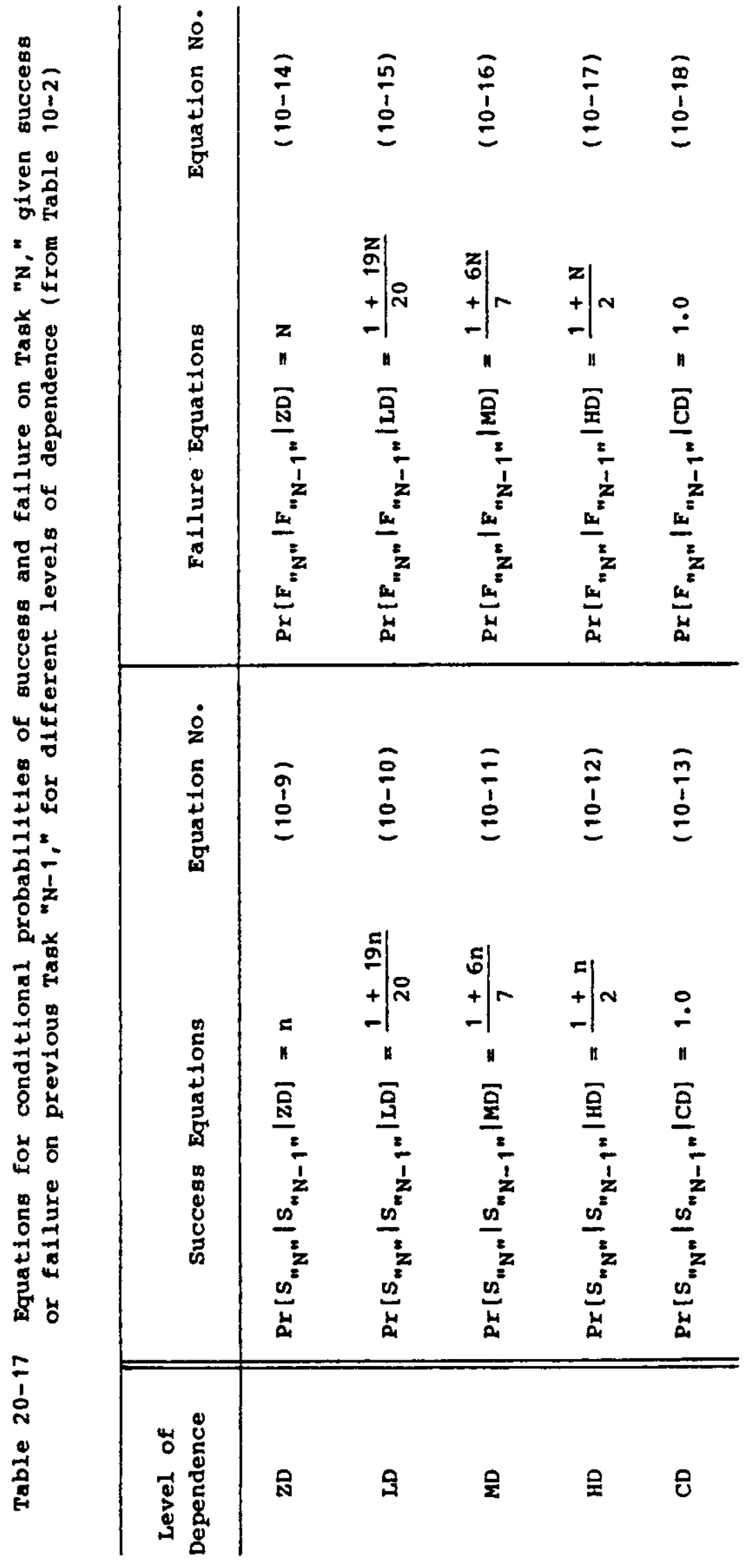


18

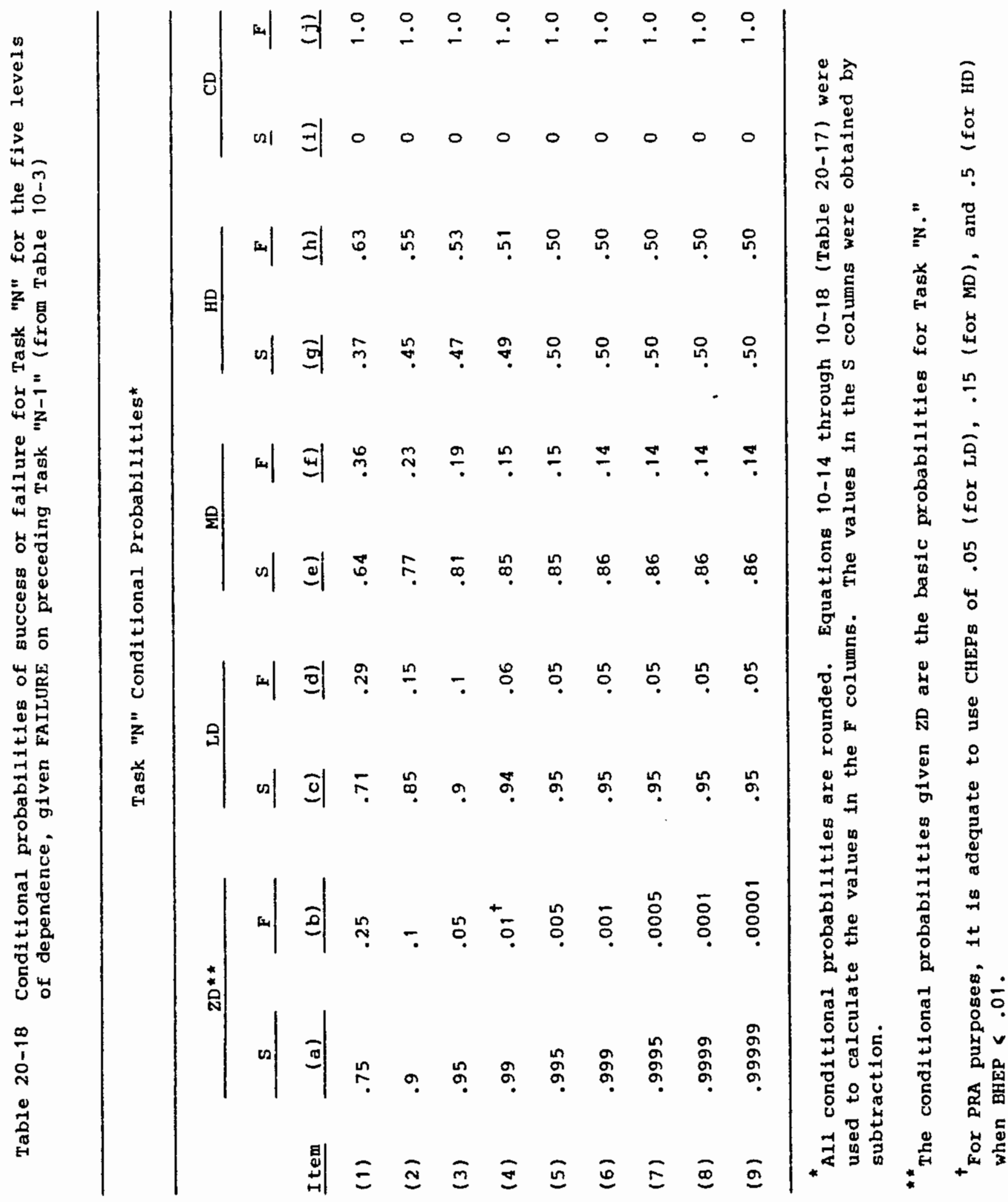




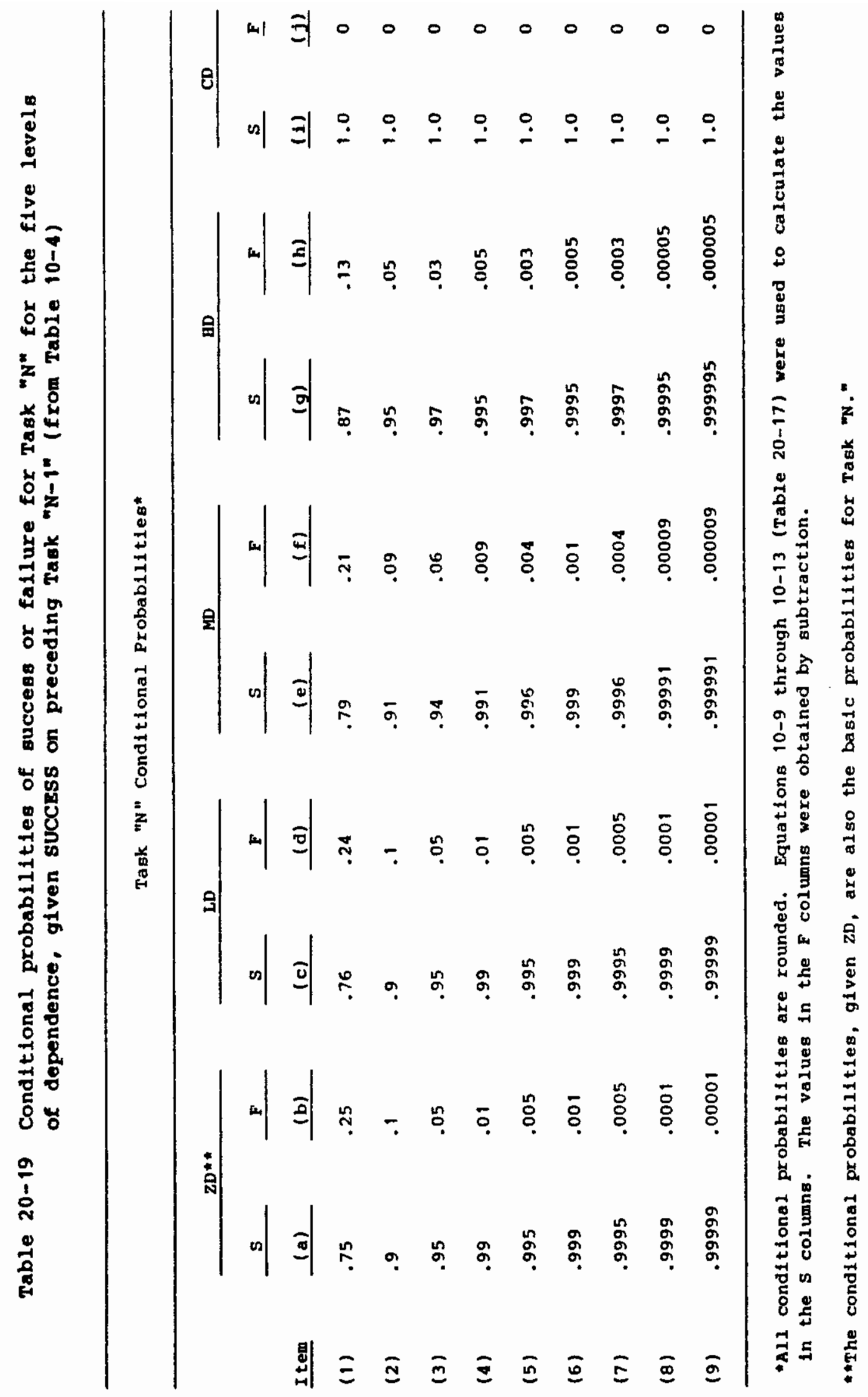


Table 20-20 General guidelines for estimating uncertainty bounds for estimated HEPs* (from Table 7-2)

Item Task and HEP Guidelines* EF $^{\dagger}$

Task consists of performance of step-by-step procedure ${ }^{+\dagger}$ conducted under routine circumstances (e.g., a test, maintenance, or calibration task); stress level is optimal:

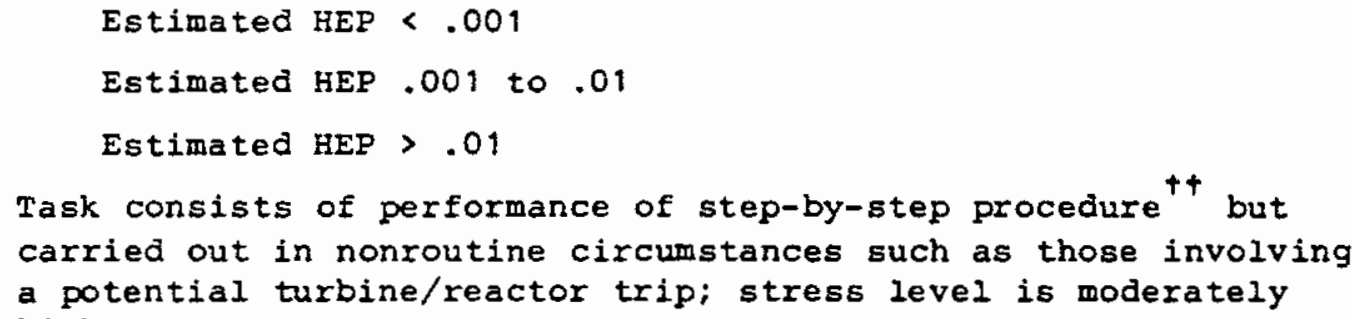

Estimated HEP > .01

Task consists of performance of step-by-step procedure ${ }^{+t^{+}}$but carried out in nonroutine circumstances such as those involving a potential turbine/reactor trip; stress level is moderately high:

$$
\text { Estimated HEP }<.001
$$

$$
\text { Estimated HEP }>.001
$$

Task consists of relatively dynamic ${ }^{+\dagger}$ interplay between operator and system indications, under routine conditions, e.g., increasing or reducing power; stress level is optimal

$$
\begin{aligned}
& \text { Estimated HEP < .001 } \\
& \text { Estimated HEP > .001 } \\
& \text { Task consists of relatively dynamic }{ }^{++} \text {interplay between operator } \\
& \text { and system indications but carried out in nonroutine circum- } \\
& \text { stances; stress level is moderately high } \\
& \text { Any task performed under extremely high stress conditions, } \\
& \text { e.g., large LocA; conditions in which the status of EsFs is not } \\
& \text { perfectly clear; or conditions in which the initial operator } \\
& \text { responses have proved to be inadequate and now severe time } \\
& \text { pressure is felt (see Ch. } 7 \text { for rationale for EF = 5) }
\end{aligned}
$$

\footnotetext{
* The estimates in this table apply to experienced personnel. The performance of novices is discussed in Chapter 18.

**For UCBs for HEPs based on the dependence model, see Table 20-21.

the highest upper bound is 1.0 .

See Appendix A to calculate the UCBs for Pr[F $F_{T}$, the total-failure term of an HRA event tree.

${ }^{+t}$ See Table 20-16 for definitions of step-by-step and dynamic procedures.
} 
Table 20-21 Approximate CHEPs and their UCBs for dependence levels* given FAILURE on the preceding task (from Table 7-3)

\begin{tabular}{|c|c|c|c|c|}
\hline & $\begin{array}{c}\text { Levels } \\
\text { of } \\
\text { Dependence }\end{array}$ & \multicolumn{2}{|r|}{ BHEPS } & \multirow[b]{2}{*}{ (c) } \\
\hline Item & & (a) & (b) & \\
\hline \multirow[t]{3}{*}{ (1) } & $Z D * *$ & $\begin{array}{l}6.01 \\
(d) \\
\end{array}$ & $\begin{array}{c}.05(E F=5) \\
(e)\end{array}$ & $\begin{array}{c}.1 \quad(E F=5) \\
(E) \\
\end{array}$ \\
\hline & & $.15 \quad(E F=5)$ & $.2(E F=5)$ & $.25 \quad(E F=5)$ \\
\hline & $\begin{array}{c}\text { Levels } \\
\text { of } \\
\text { Dependence }\end{array}$ & \multicolumn{3}{|c|}{ Nominal CHEPs and (Lower to Upper UCBs) ${ }^{\dagger}$} \\
\hline Item & & (a) & (b) & (c) \\
\hline (2) & LD & $.05(.015$ to .15$)$ & $.1 \quad(.04$ to .25$)$ & $.15(.05$ to .5$)$ \\
\hline (3) & $M D$ & $.15(.04$ to .5$)$ & $.19(.07$ to .53$)$ & $.23(.1$ to .55$)$ \\
\hline (4) & HD & $.5(.25$ to 1.0$)$ & $.53(.28$ to 1.0$)$ & $.55(.3$ to 1.0$)$ \\
\hline (5) & $\mathrm{CD}$ & $\begin{array}{c}1.0 \quad(.5 \text { to } 1.0) \\
\text { (d) }\end{array}$ & $\begin{array}{c}1.0(.53 \text { to } 1.0) \\
(\mathrm{e}) \\
\end{array}$ & $\begin{array}{c}1.0(.55 \text { to } 1.0) \\
(\mathrm{f})\end{array}$ \\
\hline (2) & LD & $.19(.05$ to .75$)$ & $.24(.06$ to 1.0$)$ & $.29(.08$ to 1.0$)$ \\
\hline (3) & MD & $.27(.1$ to .75$)$ & $.31(.1$ to 1.0$)$ & $.36(.13$ to 1.0$)$ \\
\hline (4) & $\mathrm{HD}$ & $.58(.34$ to 1.0$)$ & $.6(.36$ to 1.0$)$ & $.63(.4$ to 1.0$)$ \\
\hline (5) & $\mathrm{CD}$ & $1.0 \quad(.58$ to 1.0$)$ & $1.0(.6$ to 1.0$)$ & $1.0(.63$ to 1.0$)$ \\
\hline
\end{tabular}

*Values are rounded from calculations based on Appendix A. All values are based on skilled personnel (i.e., those with $>6$ months experience on the tasks being analyzed.

$\star *$

$Z D=$ BHEP. EFs for BHEPs should be based on Table 20-20.

Iinear interpolation between stated CHEPs (and UCBs) for values of BHEPG between those listed is adequate for most PRA studies. 
Table 20-22 Estimated probabilities that a checker will fail to detect errors made by others* (from Table 19-1)

\section{I tem}

Checking Operation

HEP EF

(1) Checking routine tasks, checker using written

15

materials (includes over-the-shoulder inspections, verifying position of locally operated valves, switches, circuit breakers, connectors, etc., and checking written lists, tags, or procedures for accuracy)

(2) Same as above, but without written materials

$\begin{array}{ll}.2 & 5 \\ .05 & 5 \\ .01 & 5 \\ .5 & 5\end{array}$

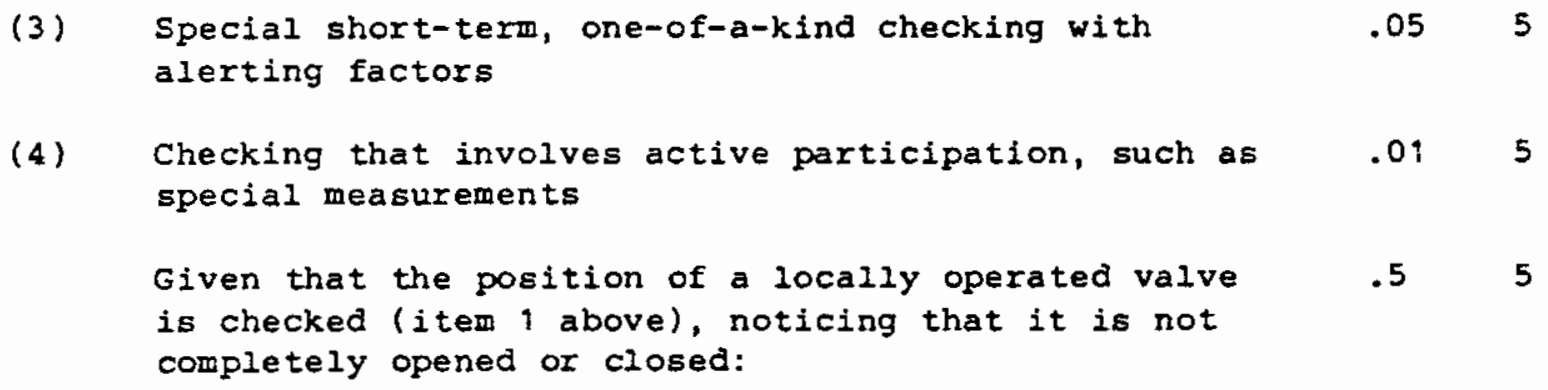

Given that the position of a locally operated valve
is checked (item 1 above), noticing that it is not completely opened or closed:

(4) Checking that involves active participation, such as .01 5 special measurements

$$
\begin{array}{lr}
\text { Position indicator** only } & .1 \\
\text { Position indicator** and a rising stem } & .5 \\
\text { Neither a position indicator* nor a rising stem } & .9 \\
\text { Checking by reader/checker of the task performer in } & .5 \\
\text { a two-man team, or checking by a second checker, } & 5
\end{array}
$$$$
\text { routine task (no credit for more than } 2 \text { checkers) }
$$$$
\text { Checking the status of equipment if that status } \quad .0015
$$

*This table applies to cases during normal operating conditions in which a person is directed to check the work performed by others either as the work is being performed or after its completion.

** A position indicator incorporates a scale that indicates the position of the valve relative to a fully opened or fully closed position. A rising stem qualifies as a position indicator if there is a scale associated with it. 


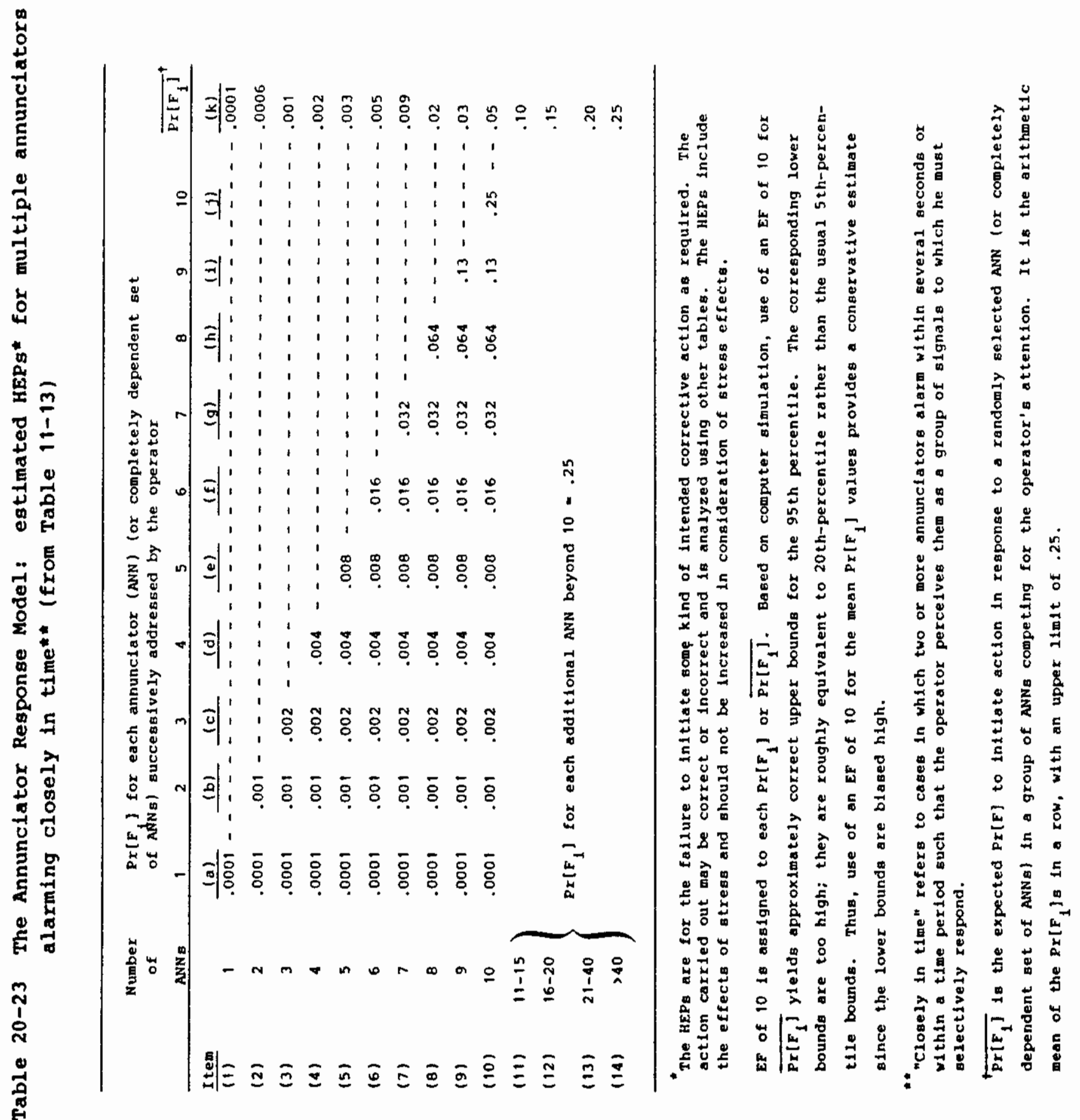


Table 20-24 Estimated HEPs for annunciated legend lights* (from Table 11-12)

\begin{tabular}{|c|c|c|c|}
\hline Item & Task & HEP & $E F$ \\
\hline (1) & $\begin{array}{l}\text { Respond* to one or more annunciated } \\
\text { legend lights }\end{array}$ & See Table $20-23$ & \\
\hline (2) & $\begin{array}{l}\text { Resume attention to a legend light } \\
\text { within } 1 \text { minute after an inter- } \\
\text { ruption (sound and blinking } \\
\text { cancelled before interruption) }\end{array}$ & .001 & 3 \\
\hline (3) & $\begin{array}{l}\text { Respond to a legend light if more } \\
\text { than } 1 \text { minute elapses after an } \\
\text { interruption (sound and blinking } \\
\text { cancelled before interruption) }\end{array}$ & .95 & 5 \\
\hline (4) & $\begin{array}{l}\text { Respond to a steady-on legend } \\
\text { light during initial audit }\end{array}$ & .90 & 5 \\
\hline (5) & $\begin{array}{l}\text { Respond to a steady-on legend } \\
\text { light during other hourly scans }\end{array}$ & .95 & 5 \\
\hline
\end{tabular}

*No written materials are used.

** "Respond" means to initiate some action in response to the indicator whether or not the action is correct. It does not include the initial acts of canceling the sound and the blinking; these are assumed to always occur. 
Table 20-25 Estimated probabilities of failure to detect one (of one) unannunciated deviant display* at each scan, when scanned hourly* (from Table 11-7)

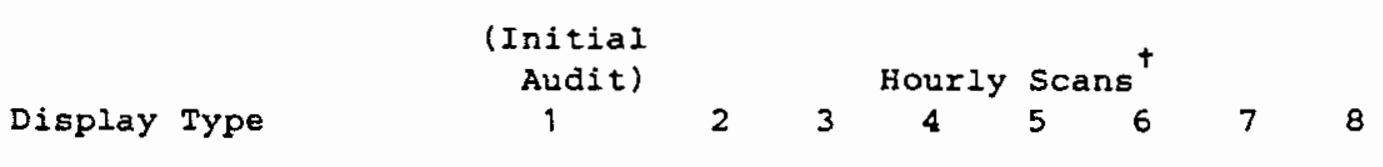

Analog meters:

with limit marks

without limit marks

6) Iegend light ${ }^{+t}$ other than annunciator ilght

Analog-type chart recorders:

$\begin{array}{llllllll}.05 & .31 & .50 & .64 & .74 & .81 & .86 & .90 \\ .15 & .47 & .67 & .80 & .87 & .92 & .95 & .97\end{array}$

$\begin{array}{llllllll}.10 & .40 & .61 & .74 & .83 & .89 & .92 & .95\end{array}$

$\begin{array}{llllllll}.30 & .58 & .75 & .85 & .91 & .94 & .97 & .98\end{array}$

$\begin{array}{llllllll}.9 & .95 & .95 & .95 & .95 & .95 & .95 & .95\end{array}$

.98

$\begin{array}{lllllll}.98 & .98 & .98 & .98 & .98 & .98 & .98\end{array}$

.99

\footnotetext{
" "One display" refers to a single display or a group of completely dependent displays, i.e., a perceptual unit. 
Table 20-26 Estimated probabilities of failing to detect at least one* of one to five unannunciated deviant displays as a function of the BHEP for detection of a single deviant display during periodic scanning** (from Table 11-6)

Number of Deviant Indications

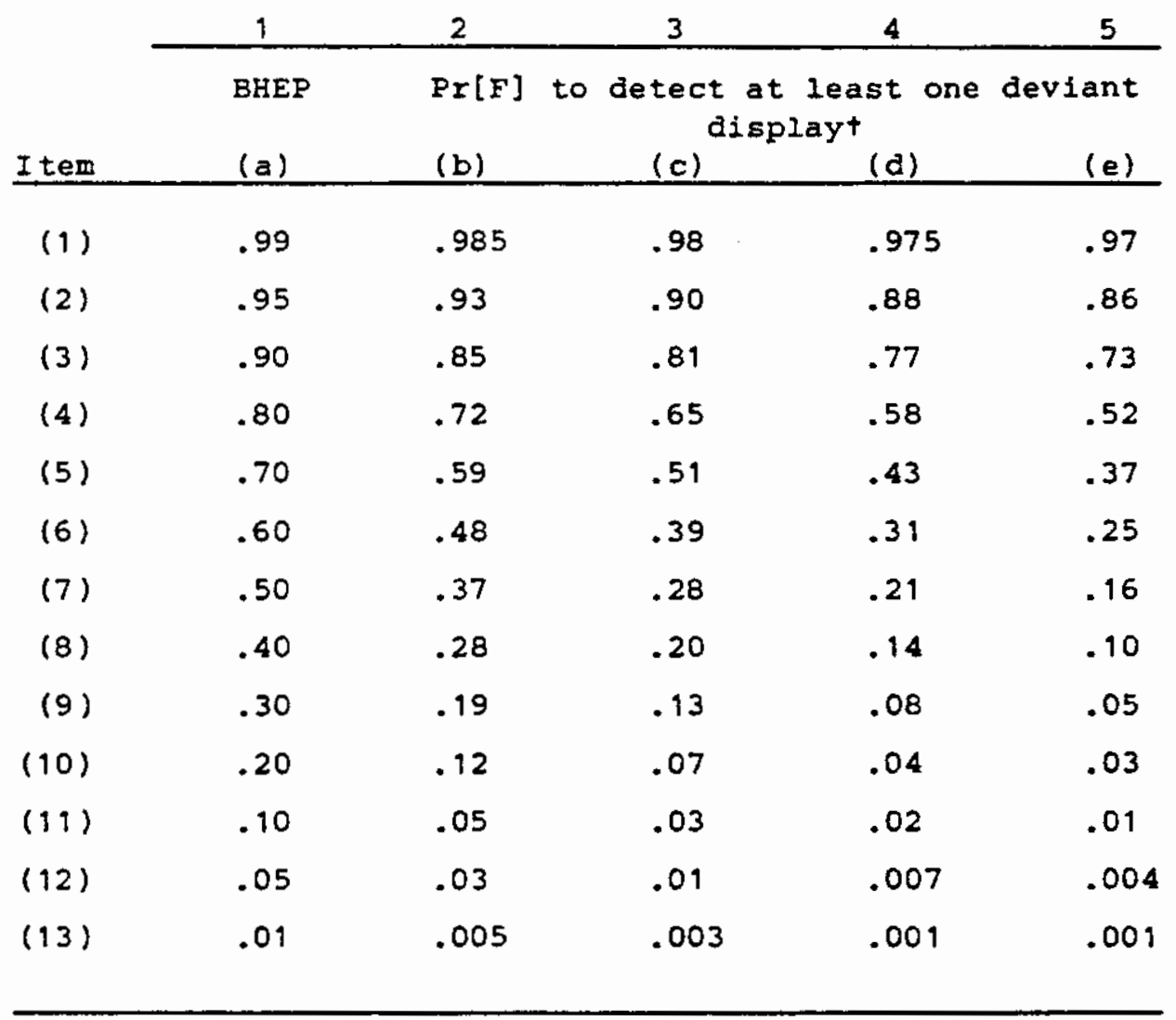

*To estimate the HEP for failure to detect other concurrent unannunciated deviant displays when one has been detected, use the HEP for the initial audit for those displays that are not functionaliy related to the display detected (from Table 20-25) and use the annunciator response model for those displays that are functionally related to the display detected (from Table 20-23). The HEPs apply when no written materials are used.

**

Except for column (a), the entries above are the complements of the entries in Table 11-5.

For EFs, refer to Table 20-20. 
Table 20-27 Estimated probabilities that the basic walk-around inspection* will fail to detect a particular deviant indication of equipment outside the control room within 30 days** (from Table 19-4)

\begin{tabular}{|c|c|c|}
\hline Item & $\begin{array}{l}\text { Number of days } \\
\text { between } \\
\text { walk-arounds } \\
\text { per inspector }\end{array}$ & $\begin{array}{l}\text { Cumulative Pr[F] } \\
\text { within } 30 \text { days } \\
\text { given one } \\
\text { inspection per shifttt }\end{array}$ \\
\hline (1) & $\begin{array}{l}1 \text { (daily walk-around } \\
\text { for each inspector) }\end{array}$ & .52 \\
\hline (2) & 2 & .25 \\
\hline (3) & 3 & .05 \\
\hline (4) & 4 & .003 \\
\hline (5) & 5 & .0002 \\
\hline (6) & 6 & .0001 \\
\hline (7) & $\begin{array}{l}7 \text { (weekly walk-around } \\
\text { for each inspector) }\end{array}$ & .0001 \\
\hline
\end{tabular}

* See Chapter 19 for the assumptions for the basic walk-around inspection. One of these assumptions is that no written procedure is used; if a written procedure is used for a walk-around, use the tables related to errors of omission and commission for performance of rule-based tasks (Figure 20-1, p 1 ).

* *

Three shifts per day are assumed. If not, use the appropriate equations in Chapter 19.

'It is assumed that all inspectors have the same number of days between walk-arounds. For other assumptions, modify the relevant equations in Chapter 19.

${ }^{+t}$ For EFs, use the procedure in Appendix $A$, or use EF $=10$ as an approximation. 

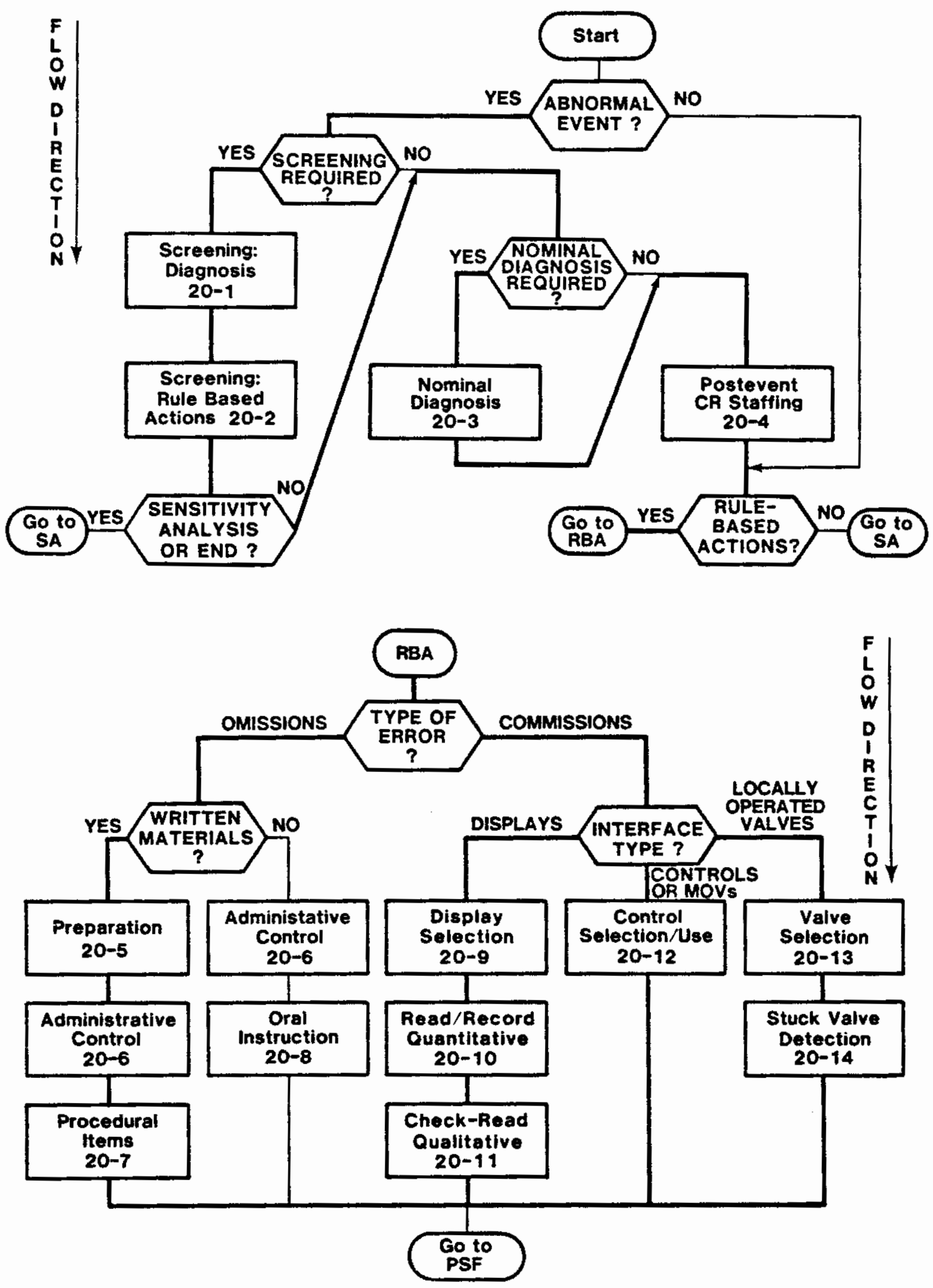

Figure 20-1 Search scheme for use of Chapter 20 tables ( $p \quad$ of 3 ). 


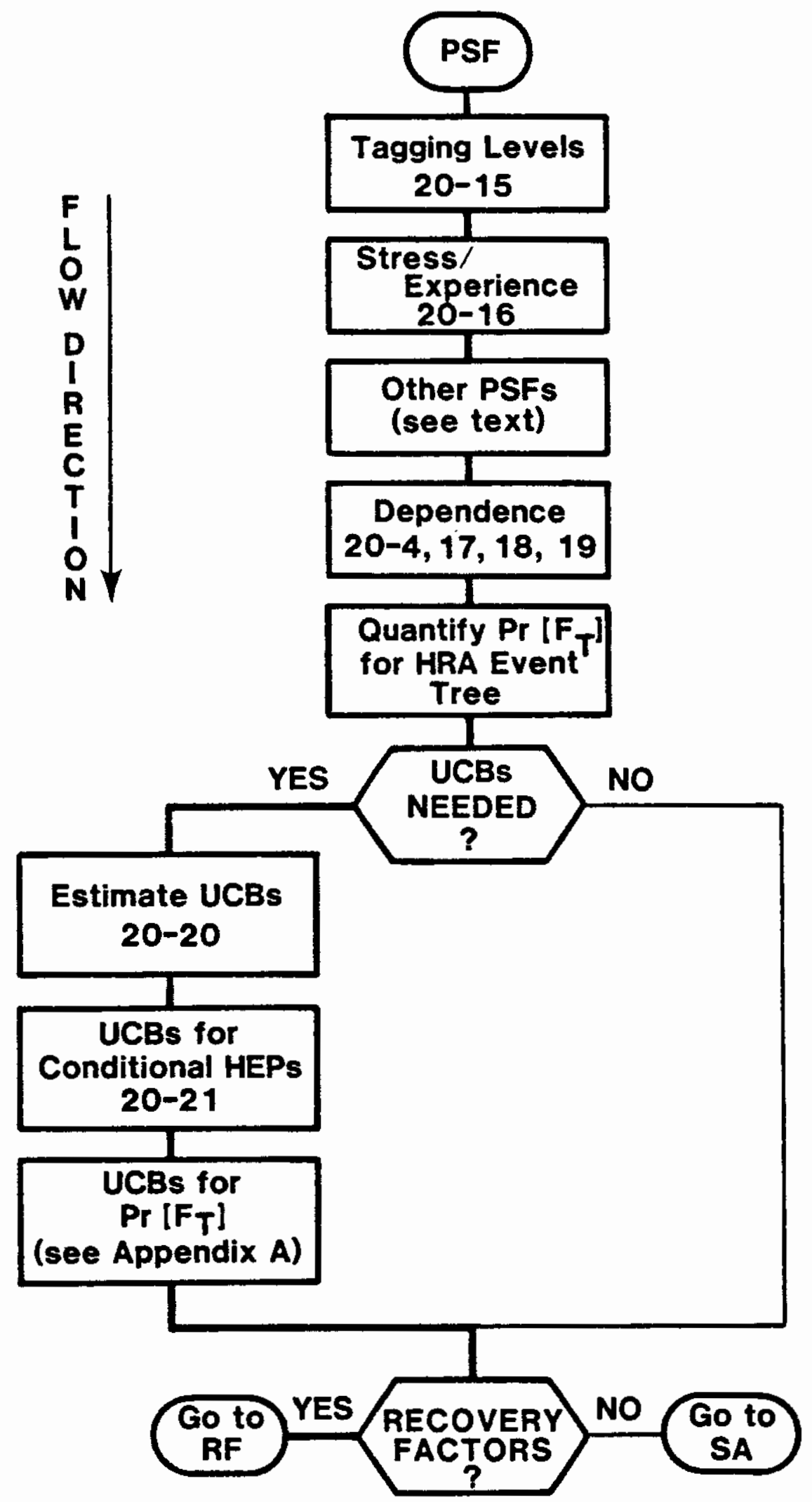

Figure 20-1 Search scheme for use of Chapter 20 tables ( $\left.\begin{array}{llll}p_{2} & \text { of } & 3\end{array}\right)$. 

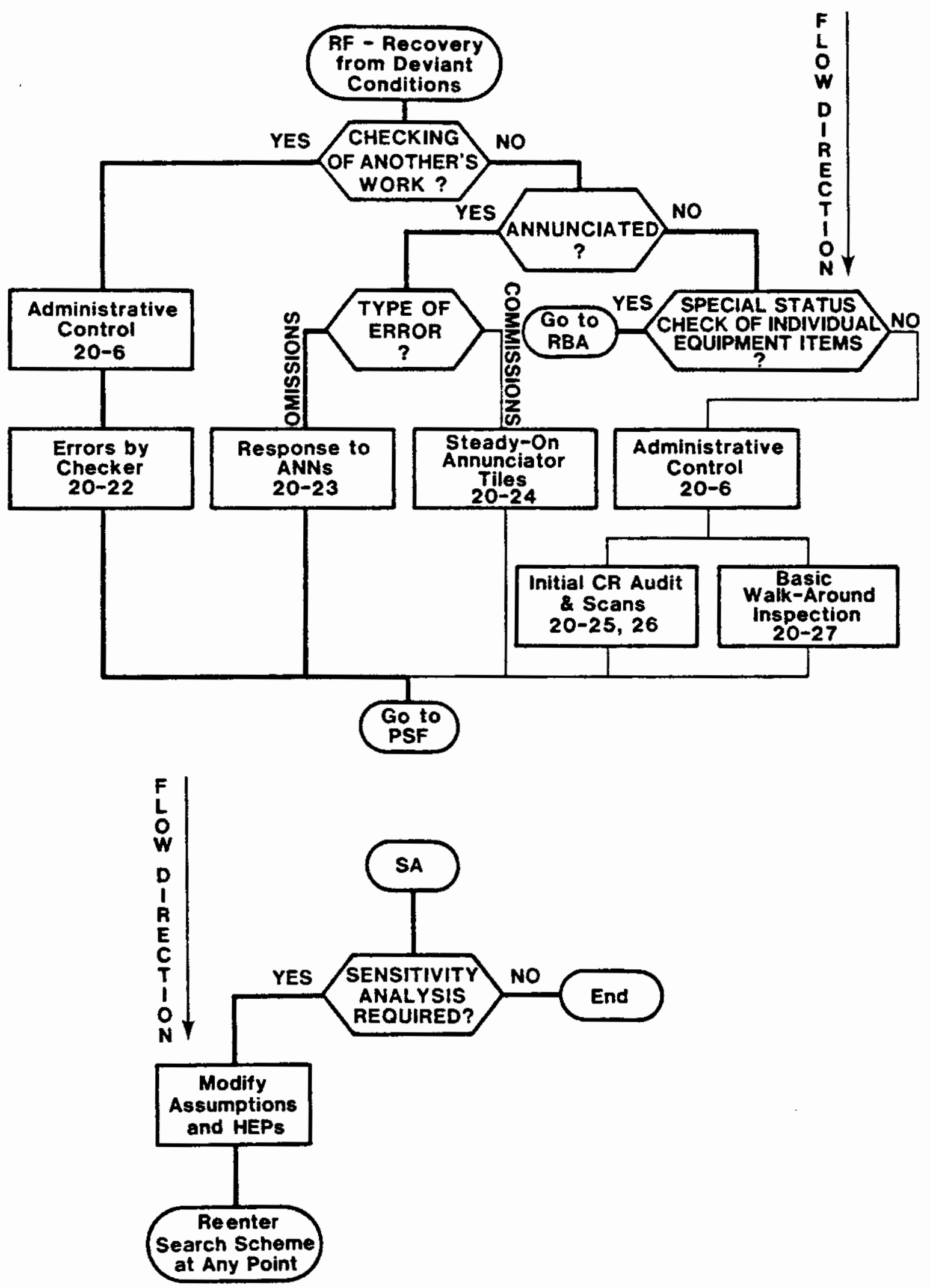

Figure 20-1 Search scheme for use of Chapter 20 tables ( $\left.\begin{array}{lll}3 & \text { of } & 3\end{array}\right)$. 


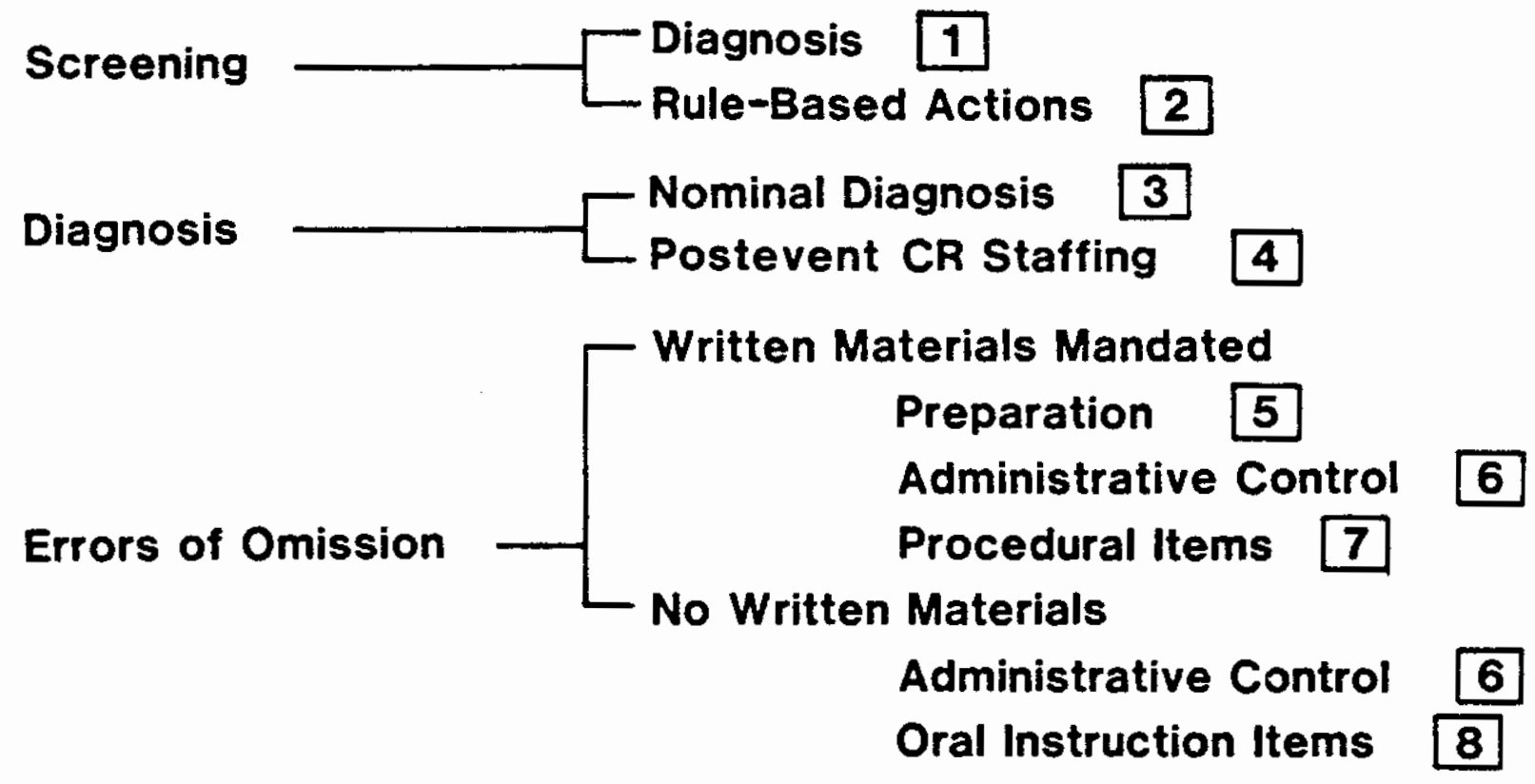

Errors of Commission $-\left[\begin{array}{ll}\text { Displays } & \begin{array}{l}\text { Display Selection } \\ \text { Read/Record Quantitative } \\ \text { Check-Read Quantitative }\end{array} \\ \text { Control \& MOV Selection \& Use } \\ \text { Locally Operated Valves }\end{array}\right.$

Valve Selection 13

Stuck Valve Detection 14

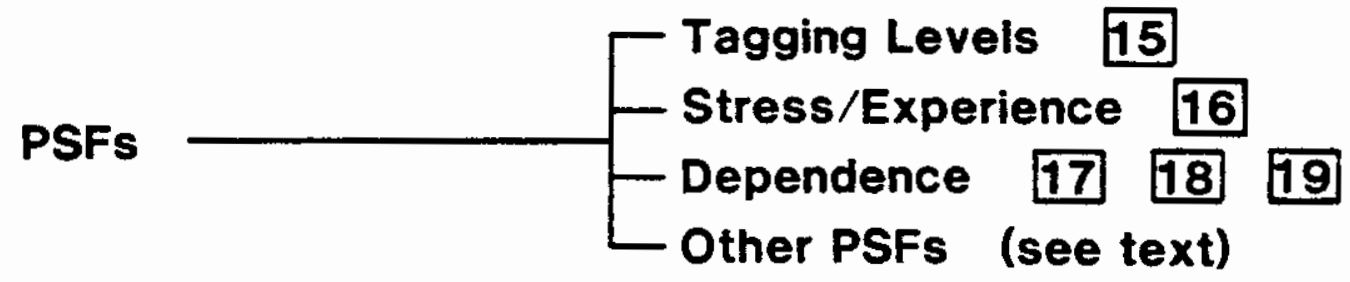

Uncertainty Bounds $-\left[\begin{array}{l}\text { Estimate UCBs } 20 \\ \text { Conditional HEPs and UCBs 21 }\end{array}\right.$

Recovery Factors $-\left[\begin{array}{l}\text { Errors by Checker } \\ \text { Annunciated Cues } \\ \text { Control Room Scanning } \\ \text { Basic Walk-Around Inspection }\end{array}\right.$

Figure 20-2 Quick reference guide to Chapter 20 tables. 
APPENDIX G 


\section{HRA Worksheets for At-Power \\ SPAR HUMAN ERROR WORKSHEET}

Plant: Initiating Event:

Basic Event : Event Coder:

\section{Basic Event Context:}

\section{Basic Event Description:}

Does this task contain a significant amount of diagnosis activity? YES $\square$ (start with Part I-Diagnosis) NO $\square$ (skip Part I - Diagnosis; start with Part II - Action) Why?

PART I. EVALUATE EACH PSF FOR DIAGNOSIS

A. Evaluate PSFs for the Diagnosis Portion of the Task, If Any.

\begin{tabular}{|c|c|c|c|}
\hline PSFs & PSF Levels & $\begin{array}{l}\text { Multiplier for } \\
\text { Diagnosis }\end{array}$ & $\begin{array}{l}\text { Please note specific reasons for } \\
\text { PSF level selection in this } \\
\text { column. }\end{array}$ \\
\hline \multirow{6}{*}{$\begin{array}{l}\text { Available } \\
\text { Time }\end{array}$} & Inadequate time & $\begin{array}{l}\mathrm{P}(\text { failure })=1.0 \square \\
\end{array}$ & \\
\hline & Barely adequate time $(\approx 2 / 3 \times$ nominal $)$ & $10 \quad \square$ & \\
\hline & Nominal time & $\square$ & \\
\hline & $\begin{array}{l}\text { Extra time (between } 1 \text { and } 2 \times \text { nominal and }> \\
\text { than } 30 \mathrm{~min} \text { ) }\end{array}$ & 0.1 & \\
\hline & Expansive time $(>2 \mathrm{x}$ nominal and $>30 \mathrm{~min})$ & 0.01 & \\
\hline & Insufficient information & $\square$ & \\
\hline \multirow{4}{*}{$\begin{array}{l}\text { Stress/ } \\
\text { Stressors }\end{array}$} & Extreme & $\square$ & \\
\hline & High & $\square$ & \\
\hline & Nominal & $\square$ & \\
\hline & Insufficient Information & $\square$ & \\
\hline \multirow[t]{5}{*}{ Complexity } & Highly complex & $\square$ & \\
\hline & Moderately complex & $\square$ & \\
\hline & Nominal & $\square$ & \\
\hline & Obvious diagnosis & 0.1 & \\
\hline & Insufficient Information & $\square$ & \\
\hline \multirow{4}{*}{$\begin{array}{l}\text { Experience/ } \\
\text { Training }\end{array}$} & Low & 10 & \\
\hline & Nominal & $\square$ & \\
\hline & High & 0.5 & \\
\hline & Insufficient Information & $\square$ & \\
\hline \multirow[t]{6}{*}{ Procedures } & Not available & 50 & \\
\hline & Incomplete & 20 & \\
\hline & Available, but poor & $\square$ & \\
\hline & Nominal & $\square$ & \\
\hline & Diagnostic/symptom oriented & 0.5 & \\
\hline & Insufficient Information & $\square$ & \\
\hline \multirow{5}{*}{$\begin{array}{l}\text { Ergonomics/ } \\
\text { HMI }\end{array}$} & Missing/Misleading & 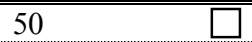 & \\
\hline & Poor & 10 & \\
\hline & Nominal & $\square$ & \\
\hline & Good $-a$ & 0.5 & \\
\hline & Insufficient Information & 1 & \\
\hline \multirow{4}{*}{$\begin{array}{l}\text { Fitness for } \\
\text { Duty }\end{array}$} & Unfit & $\mathrm{P}($ failure $)=1.0 \square$ & \\
\hline & Degraded Fitness & $5-3$ & \\
\hline & Nominal & $\square$ & \\
\hline & Insufficient Information & $\square$ & \\
\hline \multirow{4}{*}{$\begin{array}{l}\text { Work } \\
\text { Processes }\end{array}$} & Poor & $\square$ & \\
\hline & Nominal & $\square$ & \\
\hline & Good $3 n=3 n$ & 0.8 & \\
\hline & Insufficient Information & $\square$ & \\
\hline
\end{tabular}

Rev $1(1 / 20 / 04)$

Reviewer: 
Plant: Initiating Event:

Basic Event Context:

Basic Event Description:

\section{B. Calculate the Diagnosis Failure Probability.}

(1) If all PSF ratings are nominal, then the Diagnosis Failure Probability $=1.0 \mathrm{E}-2$

(2) Otherwise, the Diagnosis Failure Probability is: 1.0E-2 x Time x Stress or Stressors x Complexity x Experience or Training x Procedures x Ergonomics or HMI x Fitness for Duty x Processes

Diagnosis: $1.0 \mathrm{E}-2 \mathrm{x}$ $\mathrm{x}$ $\mathrm{x}$ $\mathrm{x}$ $\mathrm{x}$ $\mathrm{X}$ X $\mathrm{X}$

\section{Calculate the Adjustment Factor IF Negative Multiple ( $\geq 3)$ PSFs are Present.}

When 3 or more negative PSF influences are present, in lieu of the equation above, you must compute a composite PSF score used in conjunction with the adjustment factor. Negative PSFs are present anytime a multiplier greater than 1 is selected. The Nominal HEP (NHEP) is 1.0E-2 for Diagnosis. The composite PSF score is computed by multiplying all the assigned PSF values. Then the adjustment factor below is applied to compute the HEP:

$$
H E P=\frac{N H E P \cdot P S F_{\text {composite }}}{N H E P \cdot\left(P S F_{\text {composite }}-1\right)+1}
$$

Diagnosis HEP with Adjustment Factor $=$

\section{Record Final Diagnosis HEP.}

If no adjustment factor was applied, record the value from Part B as your final diagnosis HEP. If an adjustment factor was applied, record the value from Part C.

Final Diagnosis HEP $=$ 
Plant:

\section{Basic Event Context:}

Basic Event Description:

\section{Part II. EVALUATE EACH PSF FOR ACTION}

A. Evaluate PSFs for the Action Portion of the Task, If Any.

\begin{tabular}{|c|c|c|c|}
\hline PSFs & PSF Levels & $\begin{array}{l}\text { Multiplier fo } \\
\text { Action }\end{array}$ & $\begin{array}{l}\text { Please note specific reasons for } \\
\text { PSF level selection in this } \\
\text { column. }\end{array}$ \\
\hline \multirow{6}{*}{$\begin{array}{l}\text { Available } \\
\text { Time }\end{array}$} & Inadequate time & $\mathrm{P}($ failure $)=1.0$ & \\
\hline & Time available is $\approx$ the time required & $10-2$ & \\
\hline & Nominal time & 1 & \\
\hline & Time available $\geq 5 \mathrm{x}$ the time required & 0.1 & \\
\hline & Time available is $\geq 50 \mathrm{x}$ the time required & 0.01 & \\
\hline & Insufficient Information & 1 & \\
\hline \multirow{4}{*}{$\begin{array}{l}\text { Stress/ } \\
\text { Stressors }\end{array}$} & Extreme & $\overline{5}$ & \\
\hline & High & 2 & \\
\hline & Nominal $3 a n$ & 1 & \\
\hline & Insufficient Information & 1 & \\
\hline \multirow[t]{4}{*}{ Complexity } & Highly complex & $\overline{55}$ & \\
\hline & Moderately complex & 2 & \\
\hline & Nominal & 1 & \\
\hline & Insufficient Information & 1 & \\
\hline \multirow{4}{*}{$\begin{array}{l}\text { Experience/ } \\
\text { Training }\end{array}$} & Low & 3 & \\
\hline & Nominal & 1 & \\
\hline & High & 0.5 & \\
\hline & Insufficient Information & 1 & \\
\hline \multirow[t]{5}{*}{ Procedures } & Not available & 250 & \\
\hline & Incomplete & 20 & \\
\hline & Available, but poor & 5 & \\
\hline & Nominal & 1 & \\
\hline & Insufficient Information & 1 & \\
\hline \multirow{5}{*}{$\begin{array}{l}\text { Ergonomics/ } \\
\text { HMI }\end{array}$} & Missing/Misleading & $\overline{50}$ & \\
\hline & 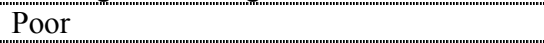 & 10 & \\
\hline & Nominal & 1 & \\
\hline & Good $-m_{n}$ & 0.5 & \\
\hline & Insufficieient Information & 1 & \\
\hline \multirow{4}{*}{$\begin{array}{l}\text { Fitness for } \\
\text { Duty }\end{array}$} & Unfit & $\mathrm{P}($ failure $)=1.0$ & \\
\hline & Degraded Fitness & 5 & \\
\hline & Nominal & 1 & \\
\hline & Insufficient Information & 1 & \\
\hline \multirow{4}{*}{$\begin{array}{l}\text { Work } \\
\text { Processes }\end{array}$} & Poor & 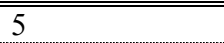 & \\
\hline & Nominal & 1 & \\
\hline & Good & 0.5 & \\
\hline & Insufficient Information & 1 & \\
\hline
\end{tabular}




\section{Basic Event Context:}

\section{Basic Event Description:}

\section{B. Calculate the Action Failure Probability.}

(1) If all PSF ratings are nominal, then the Action Failure Probability $=1.0 \mathrm{E}-3$

(2) Otherwise, the Action Failure Probability is: 1.0E-3 x Time x Stress or Stressors x Complexity x Experience or Training $\mathrm{x}$ Procedures $\mathrm{x}$ Ergonomics or HMI x Fitness for Duty $\mathrm{x}$ Processes

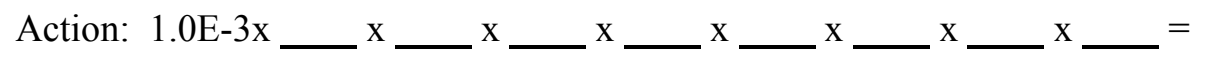

\section{Calculate the Adjustment Factor $\underline{\text { IF }}$ Negative Multiple ( $\geq 3)$ PSFs are Present.}

When 3 or more negative PSF influences are present, in lieu of the equation above, you must compute a composite PSF score used in conjunction with the adjustment factor. Negative PSFs are present anytime a multiplier greater than 1 is selected. The Nominal HEP (NHEP) is 1.0E-3 for Action. The composite PSF score is computed by multiplying all the assigned PSF values. Then the adjustment factor below is applied to compute the HEP:

$$
H E P=\frac{N H E P \cdot P S F_{\text {composite }}}{N H E P \cdot\left(P S F_{\text {composite }}-1\right)+1}
$$

\section{Record Final Action HEP.}

If no adjustment factor was applied, record the value from Part B as your final action HEP. If an adjustment factor was applied, record the value from Part $\mathrm{C}$.

$$
\text { Final Action HEP = }
$$




\section{Basic Event Context:}

\section{Basic Event Description:}

\section{PART III. CALCULATE TASK FAILURE PROBABILITY WITHOUT FORMAL DEPENDENCE (P/OD)}

Calculate the Task Failure Probability Without Formal Dependence $\left(\mathrm{P}_{\mathrm{w} / \mathrm{od}}\right)$ by adding the Diagnosis Failure Probability from Part I and the Action Failure Probability from Part II. In instances where an action is required without a diagnosis and there is no dependency, then this step is omitted.

$$
\mathrm{P}_{\mathrm{w} / \mathrm{od}}=\text { Diagnosis HEP }
$$
+ Action HEP $=$

\section{Part IV. DEPENDENCY}

For all tasks, except the first task in the sequence, use the table and formulae below to calculate the Task Failure Probability With Formal Dependence $\left(\mathrm{P}_{\mathrm{w} / \mathrm{d}}\right)$.

If there is a reason why failure on previous tasks should not be considered, such as it is impossible to take the current action unless the previous action has been properly performed, explain here:

\section{Dependency Condition Table}

\begin{tabular}{|c|c|c|c|c|c|c|}
\hline $\begin{array}{l}\text { Condition } \\
\text { Number }\end{array}$ & $\begin{array}{c}\text { Crew } \\
\text { (same or } \\
\text { different) }\end{array}$ & $\begin{array}{c}\text { Time } \\
\text { (close in time } \\
\text { or not close } \\
\text { in time) }\end{array}$ & $\begin{array}{l}\text { Location } \\
\text { (same or } \\
\text { different) }\end{array}$ & $\begin{array}{c}\text { Cues } \\
\text { (additional or } \\
\text { no } \\
\text { additional) }\end{array}$ & Dependency & $\begin{array}{l}\text { Number of Human Action Failures Rule } \\
\square \text { - Not Applicable. } \\
\text { Why? }\end{array}$ \\
\hline 1 & \multirow[t]{8}{*}{$\mathrm{S}$} & \multirow[t]{4}{*}{$\mathrm{c}$} & \multirow[t]{2}{*}{$\mathrm{S}$} & na & complete & \multirow{17}{*}{$\begin{array}{c}\text { When considering recovery in a series } \\
\text { e.g., } 2^{\text {nd }}, 3^{\text {rd }} \text {, or } 4^{\text {th }} \text { checker }\end{array}$} \\
\hline 2 & & & & $\mathrm{a}$ & complete & \\
\hline 3 & & & \multirow[t]{2}{*}{$\mathrm{d}$} & na & high & \\
\hline 4 & & & & $\mathrm{a}$ & high & \\
\hline 5 & & \multirow[t]{4}{*}{ nc } & \multirow[t]{2}{*}{$\mathrm{S}$} & na & high & \\
\hline 6 & & & & $\mathrm{a}$ & moderate & \\
\hline 7 & & & \multirow[t]{2}{*}{ d } & na & moderate & \\
\hline 8 & & & & $\mathrm{a}$ & low & \\
\hline 9 & \multirow[t]{8}{*}{$\mathrm{d}$} & \multirow[t]{4}{*}{$\mathrm{c}$} & \multirow[t]{2}{*}{$\mathrm{S}$} & na & moderate & \\
\hline 10 & & & & $\mathrm{a}$ & moderate & \\
\hline 11 & & & \multirow[t]{2}{*}{ d } & na & moderate & \\
\hline 12 & & & & $a$ & moderate & \\
\hline 13 & & \multirow[t]{4}{*}{ nc } & \multirow[t]{2}{*}{$\mathrm{S}$} & na & low & \\
\hline 14 & & & & $\mathrm{a}$ & low & \\
\hline 15 & & & \multirow[t]{2}{*}{ d } & na & low & \\
\hline 16 & & & & $\mathrm{a}$ & low & \\
\hline 17 & & & & & zero & \\
\hline
\end{tabular}

Using $\mathrm{P}_{\mathrm{w} / \mathrm{od}}=$ Probability of Task Failure Without Formal Dependence (calculated in Part III):

For Complete Dependence the probability of failure is 1 .

For High Dependence the probability of failure is $\left(1+\mathrm{P}_{\mathrm{w} / \mathrm{od}}\right) / 2$

For Moderate Dependence the probability of failure is $\left(1+6 \times \mathrm{P}_{\mathrm{w} / \mathrm{od}}\right) / 7$

For Low Dependence the probability of failure is $\left(1+19 \times \mathrm{P}_{\mathrm{w} / \mathrm{od}}\right) / 20$

For Zero Dependence the probability of failure is $\mathrm{P}_{\mathrm{w} / \mathrm{od}}$

Calculate $\mathrm{P}_{\mathrm{w} / \mathrm{d}}$ using the appropriate values:

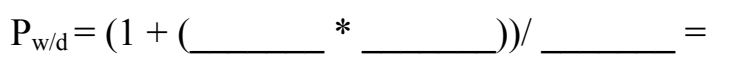

Reviewer: 


\section{HRA Worksheets for LP/SD}

SPAR HUMAN ERROR WORKSHEET

Plant:_Initiating Event:___ Basic Event :__ Event Coder:

\section{Basic Event Context:}

\section{Basic Event Description:}

Does this task contain a significant amount of diagnosis activity? YES $\square$ (start with Part I-Diagnosis) NO (skip Part I - Diagnosis; start with Part II - Action) Why?

\section{PART I. EVALUATE EACH PSF FOR DIAGNOSIS}

A. Evaluate PSFs for the Diagnosis Portion of the Task.

\begin{tabular}{|c|c|c|c|}
\hline PSFs & PSF Levels & $\begin{array}{l}\text { Multiplier for } \\
\text { Diagnosis }\end{array}$ & $\begin{array}{l}\text { Please note specific reasons for } \\
\text { PSF level selection in this } \\
\text { column. }\end{array}$ \\
\hline \multirow{6}{*}{$\begin{array}{l}\text { Available } \\
\text { Time }\end{array}$} & Inadequate time & $\begin{array}{l}\mathrm{P}(\text { failure })=1.0 \square \\
\end{array}$ & \\
\hline & Barely adequate time $(\approx 2 / 3 \times$ nominal $)$ & $10 \quad \square$ & \\
\hline & Nominal time & $\square$ & \\
\hline & $\begin{array}{l}\text { Extra time (between land } 2 \times \text { nominal and }> \\
30 \mathrm{~min} \text { ) }\end{array}$ & 0.1 & \\
\hline & Expansive time $>2 \mathrm{x}$ nominal $\&>30 \mathrm{~min}$ & 0.1 to $0.01 \quad \square$ & \\
\hline & Insufficient Information & 1 & \\
\hline \multirow{4}{*}{$\begin{array}{l}\text { Stress/ } \\
\text { Stressors }\end{array}$} & Extreme & $\bar{\square}$ & \\
\hline & High & $\square$ & \\
\hline & Nominal & $\square$ & \\
\hline & Insufficient Information & $\square$ & \\
\hline \multirow[t]{5}{*}{ Complexity } & Highly complex & $\bar{\square}$ & \\
\hline & Moderately complex & 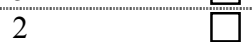 & \\
\hline & Nominal & 1 & \\
\hline & Obvious diagnosis & 0.1 & \\
\hline & Insufficient Information & 1 & \\
\hline \multirow{4}{*}{$\begin{array}{l}\text { Experience/ } \\
\text { Training }\end{array}$} & Low & 10 & \\
\hline & Nominal & $\square$ & \\
\hline & High & 0.5 & \\
\hline & Insufficient Information & 1 & \\
\hline \multirow[t]{6}{*}{ Procedures } & Not available & 50 & \\
\hline & Incomplete & 20 & \\
\hline & Available, but poor & 5 & \\
\hline & Nominal & 1 & \\
\hline & Diagnostic/symptom oriented & 0.5 & \\
\hline & Insufficient Information & 1 & \\
\hline \multirow{5}{*}{$\begin{array}{l}\text { Ergonomics/ } \\
\text { HMI }\end{array}$} & Missing/Misleading & 250 & \\
\hline & Poor & 10 & \\
\hline & Nominal & 1 & \\
\hline & Good & 0.5 & \\
\hline & Insufficient Information & $1 \quad \square$ & \\
\hline \multirow{4}{*}{$\begin{array}{l}\text { Fitness for } \\
\text { Duty }\end{array}$} & Unfit & $\begin{array}{l}\mathrm{P}(\text { failure })=1.0 \square \\
\end{array}$ & \\
\hline & Degraded Fitness & $5-\square$ & \\
\hline & Nominal & $\square$ & \\
\hline & Insufficient Information & $\square$ & \\
\hline \multirow{4}{*}{$\begin{array}{l}\text { Work } \\
\text { Processes }\end{array}$} & Poor & 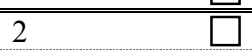 & \\
\hline & Nominal & $\square$ & \\
\hline & Good & 0.8 & \\
\hline & Insufficient Information & 1 & \\
\hline
\end{tabular}

Rev $1(1 / 20 / 04)$ 


\section{Basic Event Context:}

\section{Basic Event Description:}

\section{B. Calculate the Diagnosis Failure Probability.}

(1) If all PSF ratings are nominal, then the Diagnosis Failure Probability $=1.0 \mathrm{E}-2$

(2) Otherwise, the Diagnosis Failure Probability is: 1.0E-2 x Time x Stress or Stressors x Complexity x Experience or Training x Procedures x Ergonomics or HMI x Fitness for Duty x Processes

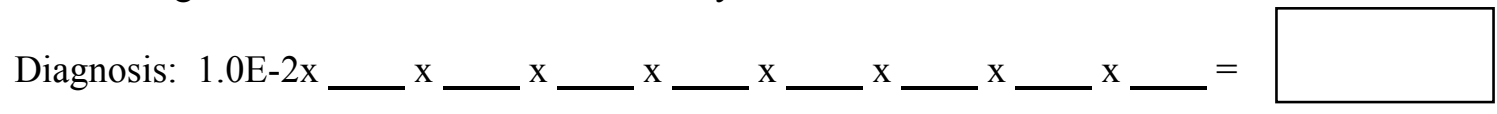

\section{Calculate the Adjustment Factor IF Negative Multiple ( $\geq 3)$ PSFs are Present.}

When 3 or more negative PSF influences are present, in lieu of the equation above, you must compute a composite PSF score used in conjunction with the adjustment factor. Negative PSFs are present anytime a multiplier greater than 1 is selected. The Nominal HEP (NHEP) is 1.0E-2 for Diagnosis. The composite PSF score is computed by multiplying all the assigned PSF values. Then the adjustment factor below is applied to compute the HEP:

$$
H E P=\frac{N H E P \cdot P S F_{\text {composite }}}{N H E P \cdot\left(P S F_{\text {composite }}-1\right)+1}
$$

$$
\text { Diagnosis HEP with Adjustment Factor }=
$$

\section{Record Final Diagnosis HEP.}

If no adjustment factor was applied, record the value from Part B as your final diagnosis HEP. If an adjustment factor was applied, record the value from Part C.

Final Diagnosis HEP = 
Plant:

Initiating Event:

Basic Event :

Event Coder:

Basic Event Context:

Basic Event Description:

Part II. EVALUATE EACH PSF FOR ACTION

A. Evaluate PSFs for the Action Portion of the Task, If Any.

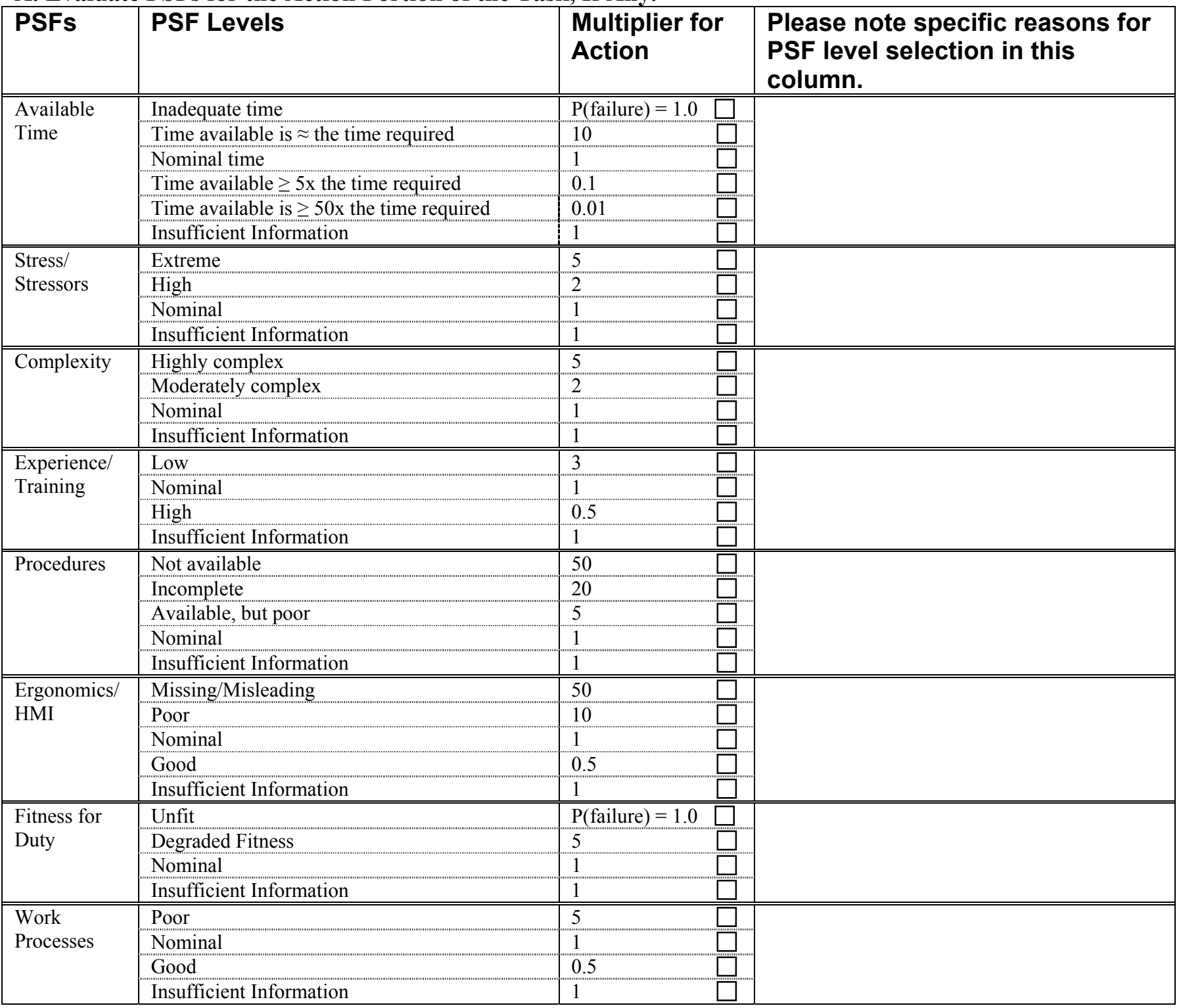

Reviewer: 


\section{Basic Event Context:}

\section{Basic Event Description:}

\section{B. Calculate the Action Failure Probability.}

(1) If all PSF ratings are nominal, then the Action Failure Probability $=1.0 \mathrm{E}-3$

(2) Otherwise, the Action Failure Probability is: 1.0E-3 x Time x Stress or Stressors x Complexity x Experience or Training $\mathrm{x}$ Procedures $\mathrm{x}$ Ergonomics or HMI x Fitness for Duty $\mathrm{x}$ Processes

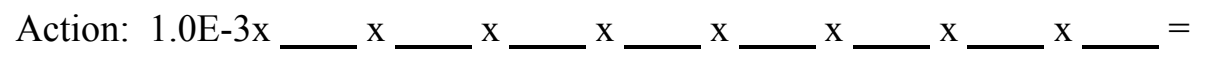

\section{Calculate the Adjustment Factor $\underline{\text { IF }}$ Negative Multiple ( $\geq 3)$ PSFs are Present.}

When 3 or more negative PSF influences are present, in lieu of the equation above, you must compute a composite PSF score used in conjunction with the adjustment factor. Negative PSFs are present anytime a multiplier greater than 1 is selected. The Nominal HEP (NHEP) is 1.0E-3 for Action. The composite PSF score is computed by multiplying all the assigned PSF values. Then the adjustment factor below is applied to compute the HEP:

$$
H E P=\frac{N H E P \cdot P S F_{\text {composite }}}{N H E P \cdot\left(P S F_{\text {composite }}-1\right)+1}
$$

\section{Record Final Action HEP.}

If no adjustment factor was applied, record the value from Part B as your final action HEP. If an adjustment factor was applied, record the value from Part C.

$$
\text { Final Action HEP }=
$$




\section{Basic Event Context:}

\section{Basic Event Description:}

\section{PART III. CALCULATE TASK FAILURE PROBABILITY WITHOUT FORMAL DEPENDENCE (P/OD)}

Calculate the Task Failure Probability Without Formal Dependence $\left(\mathrm{P}_{\mathrm{w} / \mathrm{od}}\right)$ by adding the Diagnosis Failure Probability from Part I and the Action Failure Probability from Part II. In instances where an action is required without a diagnosis and there is no dependency, then this step is omitted.

$$
\mathrm{P}_{\mathrm{w} / \mathrm{od}}=\text { Diagnosis HEP }
$$
+ Action HEP $=$

\section{Part IV. DEPENDENCY}

For all tasks, except the first task in the sequence, use the table and formulae below to calculate the Task Failure Probability With Formal Dependence $\left(\mathrm{P}_{\mathrm{w} / \mathrm{d}}\right)$.

If there is a reason why failure on previous tasks should not be considered, such as it is impossible to take the current action unless the previous action has been properly performed, explain here:

Dependency Condition Table

\begin{tabular}{|c|c|c|c|c|c|c|}
\hline $\begin{array}{c}\text { Condition } \\
\text { Number }\end{array}$ & $\begin{array}{c}\text { Crew } \\
\text { (same or } \\
\text { different) }\end{array}$ & $\begin{array}{l}\text { Time } \\
\text { (close in time } \\
\text { or not close } \\
\text { in time) }\end{array}$ & $\begin{array}{l}\text { Location } \\
\text { (same or } \\
\text { different) }\end{array}$ & $\begin{array}{c}\text { Cues } \\
\text { (additional or } \\
\text { no } \\
\text { additional) } \\
\end{array}$ & Dependency & $\begin{array}{c}\text { Number of Human Action Failures Rule } \\
\square \text { - Not Applicable. } \\
\text { Why? }\end{array}$ \\
\hline 2 & \multirow{5}{*}{$\mathrm{s}$} & \multirow{3}{*}{$\mathrm{c}$} & $\mathrm{s}$ & $\mathrm{a}$ & complete & \multirow{10}{*}{$\begin{array}{l}\text { When considering recovery in a series } \\
\text { e.g., } 2^{\text {nd }}, 3^{\text {rd }}, \text { or } 4^{\text {th }} \text { checker } \\
\text { If this error is the } 3 \text { rd error in the } \\
\text { sequence, then the dependency is at } \\
\text { least moderate. }\end{array}$} \\
\hline 3 & & & \multirow[t]{2}{*}{$\overline{\mathrm{d}}$} & na & high & \\
\hline 4 & & & & $\mathrm{a}$ & high & \\
\hline 7 & & \multirow{2}{*}{ nc } & \multirow[t]{2}{*}{$\mathrm{d}$} & na & moderate & \\
\hline 8 & & & & $\mathrm{a}$ & low & \\
\hline 9 & \multirow[t]{4}{*}{$\mathrm{d}$} & \multirow[t]{4}{*}{$\mathrm{c}$} & \multirow[t]{2}{*}{$\mathrm{s}$} & na & moderate & \\
\hline 10 & & & & $\mathrm{a}$ & moderate & \\
\hline 11 & & & \multirow[t]{2}{*}{$\mathrm{d}$} & na & moderate & \\
\hline 12 & & & & a & moderate & \\
\hline 17 & & & & & zero & \\
\hline
\end{tabular}

Using $\mathrm{P}_{\mathrm{w} / \mathrm{od}}=$ Probability of Task Failure Without Formal Dependence (calculated in Part III):

For Complete Dependence the probability of failure is 1 .

For High Dependence the probability of failure is $\left(1+\mathrm{P}_{\mathrm{w} / \mathrm{od}}\right) / 2$

For Moderate Dependence the probability of failure is $\left(1+6 \times \mathrm{P}_{\mathrm{w} / \mathrm{od}}\right) / 7$

For Low Dependence the probability of failure is $\left(1+19 \times \mathrm{P}_{\mathrm{w} / \mathrm{od}}\right) / 20$

For Zero Dependence the probability of failure is $\mathrm{P}_{\mathrm{w} / \mathrm{od}}$

Calculate $\mathrm{P}_{\mathrm{w} / \mathrm{d}}$ using the appropriate values:

$$
\mathrm{P}_{\mathrm{w} / \mathrm{d}}=(1+(
$$
* )$/$ $=$ 
APPENDIX H 


\title{
The Origins of the SPAR-H Method's Performance Shaping Factor Multipliers
}

\author{
Ronald L. Boring \& Harold S. Blackman \\ Idaho National Laboratory, Idaho Falls, Idaho, USA \\ \{ronald.boring, harold.blackman\}@inl.gov
}

\begin{abstract}
The Standardized Plant Analysis Risk-Human Reliability Analysis (SPAR-H) method has proved to be a reliable, easy-to-use method for human reliability analysis. Calculation of human error probability (HEP) rates is especially straightforward, starting with pre-defined nominal error rates for cognitive vs. action oriented tasks, and incorporating performance shaping factor (PSF) multipliers upon those nominal error rates. SPAR-H uses eight PSFs with multipliers typically corresponding to nominal, degraded, and severely degraded human performance for individual PSFs. Additionally, some PSFs feature multipliers to reflect enhanced performance. Although SPAR-H enjoys widespread use among industry and regulators, current source documents on SPAR-H such as NUREG/CR-6883 do not provide a clear account of the origin of these multipliers. The present paper redresses this shortcoming and documents the historic development of the SPAR-H PSF multipliers, from the initial use of nominal error rates, to the selection of the eight PSFs, to the mapping of multipliers to available data sources such as a Technique for Human Error Rate Prediction (THERP). Where error rates were not readily derived from THERP and other sources, expert judgment was used to extrapolate appropriate values. In documenting key background information on the multipliers, this paper provides a much needed cross-reference for human reliability practitioners and researchers of SPAR-H to validate analyses and research findings.
\end{abstract}

\section{INTRODUCTION}

The Standardized Plant Analysis Risk-Human Reliability Analysis (SPAR-H) method [1-3] was first released in 1995 as a simple-to-use approach for risk analysts to compute human error probabilities (HEPs). One way in which SPAR-H achieved simplicity was through the use of performance shaping factors (PSFs). A PSF is an aspect of the human's individual characteristics, environment, organization, or task that specifically decrements or improves human performance, thus respectively increasing or decreasing the likelihood of human error. Many early human reliability analysis (HRA) methods focused on the error likelihood of particular exemplar tasks or scenarios, whereby the risk analyst would map novel tasks or scenarios back to the pre-defined tasks or scenarios to extract an HEP. This scenario-based HRA approach (also called holistic $H R A$; see [4]) proved inflexible in application and was prone to mismatches. A different approach (also called atomistic HRA; see [4]) emerged in SPAR-H and other simplified HRA methods in which the risk analyst focused not on mapping whole tasks or scenarios but rather on mapping the applicable
PSFs within those scenarios. The use of PSFs brought greater generalizability of HRA and greater inter-analyst reliability through simplified HEP estimation processes. However, early efforts to document PSF quantification, including SPAR-H, were incomplete. In order to provide better tractability of the SPAR-H method to human performance, this article retraces the origins of SPAR-H quantification.

\section{HISTORY OF THE SPAR-H METHOD}

SPAR-H was originally called the Accident Sequence Precursor (ASP) HRA [1], in recognition of its use within the ASP program of the US Nuclear Regulatory Commission (NRC). The method was developed as a closely related alternative to two popular approaches at the time. A Technique for Human Error Rate Prediction (THERP) [5] had been formally available as a method for over ten years, although aspects of THERP were available publicly in 1975 in the US NRC's Reactor Safety Study (WASH-1400) [6] and in even earlier work by the primary author [7]. THERP analyses required considerable training and topical mastery to complete [8]. Because of the difficulty in completing a THERP analysis under strict time and resource constraints, a simplified version of THERP was commissioned in 1987 and called the Accident Sequence Evaluation Program Human Reliability Analysis Procedure (ASEP) [9]. While based on THERP, ASEP estimates diverged from those in THERP. Moreover, the technique was often emphasized as a screening HRA method, meaning its use was primarily to provide rough estimates of error likelihood for risk determination. This approach contrasted with the nuanced results offered by THERP, offering in exchange a significant time savings and greater simplicity in terms of completing an analysis.

SPAR-H was born out of THERP and ASEP as a further simplification and generalization of these two approaches. The original ASP HRA method [1] was refined in 1999 and adopted the name of the Standardized Plant Analysis Risk (SPAR) probabilistic risk assessment (PRA) models developed in support of the US NRC [2]. This latter acronym, SPAR HRA, more clearly delineated the method from ASEP. The 2005 and most recent revision [3] adopted the acronym SPAR-H, whereby the $\mathrm{H}$ signified that this method was connected specifically with HRA vs. the broader PRA focus of the SPAR models. SPAR-H was contemporary to European HRA methods such as the Human Error Assessment and Reduction Technique (HEART) [10] and the Cognitive Reliability and 


\section{Joint 8th IEEE HFPP / 13th HPRCT}

Error Analysis Method (CREAM) [11], which likewise went beyond the scenario-matching found in THERP, utilizing a series of PSFs for quantification.

SPAR-H eliminated the basic scenarios of THERP and focused on just two types of activities-processing and response. Processing referred to information processing or cognitive activities such as detection and decision making, while response referred to activities centered on behaviors and actions. This dichotomy was retained in subsequent revisions of SPAR-H but renamed diagnosis and action, respectively, to make the terms more universally understandable to a wide variety of analysts. Corresponding to these two types of activities are nominal HEPs. The context that acts upon these two types of scenarios is encompassed by a variety of PSFs, which serve as multipliers upon the nominal HEPs. This coupling of cognitive vs. behavioral activity types and PSFs affords a greater generalizability and flexibility to the analysis than can be found in scenario-based HRA. This approach is not without hazards, as acknowledged in the method documentation [3] - the data from which PSF multipliers are derived may not function in the multiplicative manner prescribed by the method, nor do the PSFs necessarily act orthogonally. The extent to which all quantitative PSF permutations and interactions in SPAR-H reflect actual human performance remains an important question for further empirical study.

\section{ORIGINS OF THE NOMINAL HEPs}

As noted above, SPAR-H features nominal HEPs for processing/diagnosis and response/action activities. These values refer to the default or average expected error rate in the absence of PSF effects. The nominal HEPs have remained constant across all three versions of SPAR-H [1-3]:

- Processing/Diagnosis: Nominal HEP = 1E-2

- $\quad$ Response/Action: Nominal HEP = 1E-3

Note that these values differ from the suggested nominal HEP in THERP [5] and ASEP [9], which is 3E-2. This divergence is attributed to the disambiguation of cognitive and behavioral activities in SPAR-H. The nominal HEP for processing/ diagnosis activities is based on the value found in THERP Table 20-1, Item 4, corresponding to the median HEP for a control room diagnosis task within 30 minutes. This follows the socalled 30-minute rule in control room activity - a general rule for how long operators should have available before they are required to take action [12]. The response/action HEP was derived from WASH-1400 [6] and numerous representative action tasks in THERP [5]. In WASH-1400, Appendix III, Table III 6-1, the erroneous activation of a switch, assuming no decision error, is estimated to be 1E-3. This corresponds to an archetypical nominal response/action in SPAR-H. THERP provides similar examples of response/action activities calibrated to an HEP of 1 E-3:

- Incorrectly following a written procedure step (Table 20-7, Item 1)
- Incorrectly selecting an unannunciated display from similar-appearing displays (Table 20-9, Item 3)

- Incorrectly "check-reading" digital indicators (Table 20-11, Item 1) or analog meters (Table 20-11, Item 2)

- Inadvertently activating a control arranged in a welldelineated functional group (Table 20-12, Item 3)

- Incorrectly selecting or activating a locally operated valve that is clearly labeled and set apart from other valves (Table 20-13, Item 1)

\section{ORIGINS OF THE PSFs AND MULTIPLIERS}

\section{A. 1995 SPAR-H Version}

The 1995 version of SPAR-H [1] included six PSFs, then known as operational factors. The selection of these six PSFs was based on the description of a cognitive model followed by the identification of factors known in the psychological literature to affect each step of that model. Using expert judgment by subject matter experts in nuclear power plant operations, this list was parsed into the six PSFs deemed to have the most relevance to and impact on human performance in terms of detection, perception, decision making, and actions in nuclear power plant operations. It is erroneous to conclude that this list of PSFs was intended to be exhaustive, although it was intended to be more complete than prior efforts in that it began from a basic cognitive model. The six PSFs were intended to represent the factors that could influence human performance, allowing a reasonable generalizability across situations and for which data could be extracted from THERP.

The six PSFs and accompanying HEPs are featured in Table 1. Each PSF features levels of effect, corresponding to different multipliers on the nominal HEP. Note that SPAR-H provides multipliers for each PSF (shown in parentheses in Table 1), not final or composite HEP values. However, the relationship between SPAR-H and THERP is best expressed in terms of the comparison of HEP values.

THERP does not clearly distinguish between processing/ diagnosis and response/action HEPs. For this reason, an HEP match is usually only possible between THERP HEP values and either processing/diagnosis or response/action HEPs in SPAR$\mathrm{H}$, but not both. Generalizing to the other case in SPAR-H is easy - in the 1995 version of SPAR-H, the PSF multipliers are identical for processing/diagnosis and response/action. Therefore, the only difference between processing/diagnosis and response/action HEPs is that processing/diagnosis HEPs are greater by a factor of 10 .

Note that for the four initial PSFs-Complexity/Stress/ Workload, Experience/Training, Procedures, and Ergonomicsall PSF multiplier levels are directly linked to THERP values. The original SPAR-H development team utilized expert judgment to arrive at the best mapping of a THERP task or scenario item to the generalized SPAR-H PSF level. This mapping was subject to revision as experience was gained using SPAR-H in practice and as additional insights on the PSF level definitions were gained. The 1995 mapping of SPAR-H PSFs to THERP task types is as follows:

Complexity, Stress, and Workload. The multipliers for this PSF are taken from representative values in THERP Tables 20- 
Joint 8th IEEE HFPP / 13th HPRCT

TABLE I. MAPPING OF ASP HRA (1995) TO THERP

\begin{tabular}{|c|c|c|c|c|c|c|}
\hline \multicolumn{5}{|c|}{ ASP HRA/SPAR-H (1995) } & \multicolumn{2}{|c|}{ THERP } \\
\hline PSF & $\begin{array}{c}\text { PSF } \\
\text { Category }\end{array}$ & $\begin{array}{c}\text { PSF } \\
\text { Level }\end{array}$ & $\begin{array}{c}\text { Processing } \\
\text { HEP }^{1}\end{array}$ & $\begin{array}{c}\text { Response } \\
\text { HEP }^{1}\end{array}$ & $\begin{array}{c}\text { HEP for } \\
\text { Processing }^{2}\end{array}$ & $\begin{array}{l}\text { HEP for } \\
\text { Response }^{2}\end{array}$ \\
\hline \multirow[t]{6}{*}{ Complexity, Stress, and Workload } & \multirow[t]{3}{*}{$\begin{array}{l}\text { High Threat } \\
\text { and Stress }\end{array}$} & $\begin{array}{l}\text { Inadequate } \\
\text { Time }\end{array}$ & $1.0(\infty)$ & $1.0(\infty)$ & & $1.0(20-1,1)$ \\
\hline & & $\begin{array}{l}\text { Adequate } \\
\text { Time }\end{array}$ & $0.05(5)$ & $0.005(5)$ & & $0.005(20-23,6)$ \\
\hline & & $\begin{array}{c}\text { Expansive } \\
\text { Time }\end{array}$ & $0.02(2)$ & $0.002(2)$ & & $0.002(20-23,4)$ \\
\hline & \multirow[t]{3}{*}{$\begin{array}{l}\text { Low Threat } \\
\text { and Stress }\end{array}$} & $\begin{array}{c}\text { Inadequate } \\
\text { Time }\end{array}$ & $1.0(\infty)$ & $1.0(\infty)$ & $1,0(20-1,1)$ & \\
\hline & & $\begin{array}{l}\text { Adequate } \\
\text { Time }\end{array}$ & $0.01(1)$ & $0.001(1)$ & $0.01(20-1,4)$ & \\
\hline & & $\begin{array}{c}\text { Expansive } \\
\text { Time } \\
\end{array}$ & $0.01(1)$ & $0.001(1)$ & $0.001(20-1,5)$ & \\
\hline \multirow[t]{4}{*}{ Experience/ Training } & \multirow[t]{2}{*}{ Low Experience } & $\begin{array}{c}\text { Poor } \\
\text { Training } \\
\end{array}$ & $0.1(10)$ & $0.01(10)$ & $10 \mathrm{x}(20-16,5)^{3}$ & \\
\hline & & $\begin{array}{c}\text { Good } \\
\text { Training }\end{array}$ & $0.01(1)$ & $0.001(1)$ & $1 \times(20-16,2)^{3}$ & \\
\hline & \multirow[t]{2}{*}{ High Experience } & $\begin{array}{c}\text { Poor } \\
\text { Training }\end{array}$ & $0.05(5)$ & $0.005(5)$ & $5 \times(20-16,5)^{3}$ & \\
\hline & & $\begin{array}{c}\text { Good } \\
\text { Training }\end{array}$ & $0.005(0.5)$ & $0.0005(0.5)$ & $0.5 \times(20-16)^{4}$ & \\
\hline \multirow[t]{3}{*}{ Procedures } & Procedures Absent & N/A & $0.1(10)$ & $0.01(10)$ & $2 \times(20-22,2)^{5}$ & \\
\hline & \multirow[t]{2}{*}{ Procedures Present } & $\begin{array}{c}\text { Poor } \\
\text { Procedures }\end{array}$ & $0.05(5)$ & $0.005(5)$ & $0.05(20-7,5)$ & $0.005(20-6,9)$ \\
\hline & & $\begin{array}{c}\text { Good } \\
\text { Procedures }\end{array}$ & $0.01(1)$ & $0.001(1)$ & & $0.001(20-7,1)$ \\
\hline \multirow[t]{6}{*}{ Ergonomics } & \multirow[t]{2}{*}{ Old Plant } & $\begin{array}{c}\text { Poor } \\
\text { Ergonomics }\end{array}$ & $0.05(5)$ & $0.005(5)$ & & $0.005(20-12,12)$ \\
\hline & & $\begin{array}{c}\text { Good } \\
\text { Ergonomics }\end{array}$ & $0.01(1)$ & $0.001(1)$ & & $0.001(20-12,3)$ \\
\hline & \multirow[t]{2}{*}{ Retrofit Plant } & $\begin{array}{c}\text { Poor } \\
\text { Ergonomics }\end{array}$ & $0.03(3)$ & $0.003(3)$ & & $0.003(20-12,2)$ \\
\hline & & $\begin{array}{c}\text { Good } \\
\text { Ergonomics }\end{array}$ & $0.007(0.7)$ & $0.0007(0.7)$ & & $0.0005(20-12,5)$ \\
\hline & \multirow[t]{2}{*}{ New Plant } & $\begin{array}{c}\text { Poor } \\
\text { Ergonomics }\end{array}$ & $0.02(2)$ & $0.002(2)$ & & $0.003(20-9,4)$ \\
\hline & & $\begin{array}{c}\text { Good } \\
\text { Ergonomics } \\
\end{array}$ & $0.004(0.4)$ & $0.0004(0.4)$ & & $0.0005(20-9,1)$ \\
\hline \multirow[t]{2}{*}{ Fitness for Duty } & Unfit & N/A & $0.25(25)$ & $0.025(25)$ & & \\
\hline & Fit & N/A & $0.01(1)$ & $0.001(1)$ & & \\
\hline \multirow[t]{2}{*}{ Crew Dynamics } & $\begin{array}{l}\text { Poor Crew } \\
\text { Dynamics }\end{array}$ & N/A & $0.1(10)$ & $0.01(10)$ & & \\
\hline & $\begin{array}{c}\text { Good Crew } \\
\text { Dynamics }\end{array}$ & N/A & $0.01(1)$ & $0.001(1)$ & & \\
\hline
\end{tabular}

1 and 20-23. Table 20-1 represents a diagnosis within different time intervals by control room personnel for abnormal events annunciated closely in time. Table 20-23 represents a related occurrence - the time to take an action for multiple simultaneous annunciators. Thus, response/ action values are primarily taken from Table $20-23$, while processing/diagnosis values are from Table 20-1. Note that the value for "Inadequate Time" for both processing/ diagnosis and response/action is taken from THERP Table 20-1, Item 1, which sets the HEP equal to 1.0 when there is inadequate time. Adequate time for "Low Threat and Stress" is assumed to be equivalent to having 30 minutes to complete the task (see discussion above on Origins of the Nominal HEPs). Having more time than 30 minutes corresponds to "Expansive Time" but is not credited with a different multiplier in SPAR-H, resulting in slightly more 


\section{Joint 8th IEEE HFPP / 13th HPRCT}

conservative values than THERP. For the "High Threat and Stress" case, the 30-minute rule is applied again. It is assumed a crew will have sufficient time to address up to four annunciators in those 30-minutes (THERP Table 2023, Item 4), corresponding to "Expansive Time" in SPAR$\mathrm{H}$. The crew will generally find they have "Adequate Time" to handle up to six such annunciators (THERP Table 20-23, Item 6). With increased annunciators beyond this point, the crew may find itself with "Inadequate Time" to respond to the annunciators.

Experience and Training. Experience and training is handled in THERP as a function of stress (Table 20-16), with separate levels of stress for skilled and novice operators. The difference between the effect of stress for skilled and novice people varies for action tasks between a factor of one for very low stress to a factor of five (skilled) and ten (novice) for moderately high or extremely high stress. These differences serve as the basis for the SPAR-H Experience and Training PSF levels.

Procedures. HEPs for Procedures involving action tasks in SPAR-H mirror the HEPs found in THERP across Tables 20-6, 20-7, and 20-22. Although THERP Chapter 15 [5] identifies the nominal HEP for written procedures to be 0.003 , a careful analysis suggests that this value assumes a long procedure. Because procedures often do not fit THERP's criterion for a long procedure (with more than 10 steps), SPAR-H adopts as its nominal value the THERP HEP for short procedures, which is 0.001 . As more deficiencies are identified with procedures or procedure use, the HEP value increments. SPAR-H adopts the step increases in HEP values found in THERP Table 20-7 although has slightly different definitions for each grade. The absence of procedures is handled in THERP Table 20-22, Items 1 and 2, which contrast performance during checking activities when procedures are available and when they are not. The lack of written materials, specifically procedures, suggests a twofold decrease in performance.

Ergonomics. The various levels of the Ergonomics PSF for response/action tasks in SPAR-H are a composite of effects documented in Chapter 14 of THERP [5]. The SPAR-H PSF is focused on crew interaction with instruments and controls but also includes perceptual aspects of displays covered in Chapter 11 of THERP, and manual control operations found in Chapter 13. Ergonomics PSF level multipliers for old and retrofit plants primarily follow those values found in THERP Table 20-12 for errors of commission in operating manual controls, a response/action activity in SPAR-H. Ergonomics PSF level multipliers for new plants are derived from values found in THERP Table 20-9 for erroneously selecting unannunciated displays, although the THERP values are slightly more conservative than those found in SPAR-H.

Fitness for Duty and Crew Dynamics. The two remaining PSFs-Fitness for Duty and Crew Dynamics - were not readily discernable from THERP as a primary data source. For Fitness for Duty, little empirical evidence was available to suggest distinct levels of degraded fitness. The effects of
Fitness for Duty were, of course, well known across industries and had served as the most significant contributor to well-known accidents. As such, the SPAR-H method developers adopted a conservative screening value. In cases where Fitness for Duty should come into question, a multiplier of 25 was applied, resulting in a minimal overall HEP equal to 0.25 for processing/diagnosis tasks and 0.025 for response/action tasks.

The Crew Dynamics PSF encompassed communications and team interaction in command and control situations, which had been explored in human factors research studies but had not been linked directly back to levels of human reliability. As such, the SPAR-H method developers likened poor Crew Dynamics to situations in which there is poor training or a lack of procedures. Absent good communications especially between the shift supervisor and the reactor operator, the effect on performance is similar to what would be expected of a crew that was inadequately trained or did not have procedures to follow. Like the "Poor Training" PSF level for crews with Low Experience and like the "Procedures Absent" PSF level, "Poor Crew Dynamics" was given a multiplier equal to 10 .

\section{B. 1999 and 2005 SPAR-H Revisions}

As noted earlier, the 1999 revision of SPAR-H [2] saw adoption of the name SPAR HRA method and a terminological shift from processing to diagnosis and from response to action. These changes were carried forward to the 2005 revision, by which time the method was called SPAR-H [3]. In terms of PSFs and PSF multipliers, the 1999 and 2005 revisions of SPAR-H [2-3] are almost identical. Both feature eight PSFs. The original single PSF entitled Complexity/Stress/Workload was deconstructed into three separate PSFs-Available Time, Stress and Stressors, and Complexity. New PSF levels and multipliers were split from the single set of PSF levels and multipliers, and, where required, the original mappings to THERP were revised.

Beginning with the 1999 SPAR-H revision, a number of new PSF levels were added that accounted for the possible positive influence of PSFs on human performance [13]. These multipliers were assigned values less than 1.0, effectively decreasing the HEP below the nominal HEP level when incorporated in the quantification. At the time THERP was developed, positive influences on human performance were not captured, and THERP provides no ready formula for crediting such influences. Therefore, it was necessary to extrapolate these positive influences to arrive at a new set of multipliers. Such values were inferred using expert judgment and do not have a direct link back to THERP or to empirical data. To avoid over-crediting such positive influences, the multipliers are conservative and have a negligible effect in decrementing the HEP.

The 2005 revision of SPAR-H [3] added two notable refinements to the 1999 revision. A new level was added to the Procedures PSF: "Incomplete" was inserted between "Not Available" and "Available but Poor," thus infilling a sizeable gap in accounting for procedural quality. The 2005 


\section{Joint 8th IEEE HFPP / 13th HPRCT}

revision also added a second set of worksheets. To account for possible differences between At Power conditions and Low Power and Shutdown conditions, separate SPAR-H worksheets were created for each condition. While extensive documentation on the differences between At Power and Low Power and Shutdown is provided with the 2005 revision, currently, the only difference between these worksheets is in their definition of the Available Time PSF level entitled "Expansive Time." Because Low Power and Shutdown activities may benefit from the absence of the type of time pressure found during At Power operations, this multiplier is offered as a range between 0.1 and 0.01 for Processing/Diagnosis activities. The lower value is used in cases where little time pressure exists, for example, due to a planned extended maintenance outage.

A comparison of the PSF multipliers in the 1995 and 2005 versions of SPAR-H is found in Table 2. The current multipliers and their relationship to THERP are detailed in Table 3. Notable recalibrations of the multipliers are highlighted below.

Available Time. This new SPAR-H PSF aligns with THERP Table 20-1, which covers diagnosis of the first event in an abnormal event for different time durations. "Inadequate Time" in SPAR-H corresponds to "diagnosis within the first minute after the initiation of the abnormal event" in THERP (Item 1). "Barely Adequate Time" in SPAR-H corresponds to diagnosis within 20 minutes (Item 3). "Nominal Time" in SPAR-H corresponds to a diagnosis time within 30 minutes in THERP (Item 4). "Extra Time" in SPAR-H corresponds to a diagnosis time within one hour in THERP (Item 5). Finally, "Expansive Time" in SPAR-H corresponds to a diagnosis within one day in THERP (Item 6).

Stress and Stressors. Note that SPAR-H groups internal and external (e.g., environmental) stress into a single PSF, which maps to THERP's stress PSF (Table 20-16). This is consistent with THERP's treatment of environmental stressors (i.e., temperature, humidity, air quality, noise and vibration, illumination, and degree of general cleanliness) and physiological stressors (e.g., radiation exposure) under its Stress PSF. The THERP stress multipliers specifically for skilled personnel are used directly in SPAR-H. "Extreme Stress" in SPAR-H corresponds to Extremely High (Threat Stress) for step-by-step tasks in THERP (Item 6). "High Stress" in SPAR-H corresponds to Moderately High (Heavy Task Load) stress for step-by-step tasks in THERP (Item 4). "Nominal Stress" in SPAR-H is equivalent to Optimum stress for step-by-step tasks (Item 3) or dynamic tasks (Item 3 ) in THERP. Note that THERP considers the effects of inadequate stress (primarily due to inadequate arousal), which are not addressed in SPAR-H. THERP sets the HEP for extremely high stress during diagnosis at 0.25. SPAR-H retains the multipliers even in extreme stress, resulting in an HEP equal to 0.05 , making THERP more conservative for extreme stress diagnosis tasks. However, it is noted in Chapter 17 of THERP [5] that there is large variability associated with extreme stress conditions. Further, THERP notes a paucity of data on performance during extreme stress conditions owing to the difficulty and ethical considerations in conducting such research. In light of the uncertainties associated with performance under extreme stress, SPAR-H balances crediting the operator and acknowledging risk.

Complexity. THERP does not directly treat complexity, which is newly treated as a PSF in SPAR-H. THERP does, however, cover a number of tasks involving complexity. The best direct match to complexity in THERP occurs in the operator response to simultaneous alarms (Table 20-23), which is included as part of the extended definition of complexity in the SPAR-H NUREG [3]. Correct response to a single alarm is given an HEP equal to 0.001 in THERP (Table 20-23, Item 1), while correct response to three alarms is deemed to have an HEP equal to 0.001 (Table 20-23, Item 3 ). This latter point is calibrated as the nominal HEP for action tasks in SPAR-H. For significantly fewer alarms, there is an enhancing effect of one order of magnitude, which is credited in SPAR-H for tasks with obvious diagnosis. The deleterious effects of complexity captured by SPAR-H are anchored to two additional points along THERP Table 20-23. Moderately complex tasks in SPAR-H are anchored equivalent to tasks involving four simultaneous alarms (Table 20-23, Item 4), producing an HEP equal to 0.002 for action tasks. Highly complex tasks are curve-fitted to the equivalent of six alarms (Table 20-23, Item 6), with an HEP equal to 0.005 .

Procedures. THERP does not explicitly provide values for symptom-oriented procedures. In SPAR-H, the diagnosis PSF for procedures credits performance enhancement for procedures that are optimized by being symptom oriented. The positive influence is extrapolated on the distribution plot from the negative influence values.

Ergonomics and Human-Machine Interface (HMI). The nominal effect of Ergonomics and HMI corresponds to the "clearly and unambiguously labeled" HEP equal to 0.001 in Table 20-13, Item 1. While THERP offers five grades of degradation for the interface, SPAR-H adopts the value from Table 20-13, Item 5 ("unclearly or ambiguously labeled") with an HEP equal to 0.01 for the poor level of the PSF. SPAR-H includes a final PSF level corresponding to missing or misleading aspects of the interface, which is not found in THERP. To consider the magnitude of such an effect, SPAR-H adopts the worst effect HEP found in THERP for interface issues, found in Table 20-12, Item 6, with an HEP equal to 0.05 . This condition corresponds to interfaces in which the design "...violates a strong population stereotype and operating conditions are normal" [5] for an error of commission in operating manual controls. Note that there exists a related HEP that is an order of magnitude stronger (Table 20-12, Item 7), but this HEP incorporates a significant consideration of stress, which is handled by a separate PSF in SPAR-H.

Fitness for Duty and Work Processes. The PSFs for Fitness for Duty and Crew Dynamics were significantly refined in the 1999 revision of SPAR-H. The authors referred particularly to HEART [10] for data, an HRA 
Joint 8th IEEE HFPP / 13th HPRCT

TABLE II. COMPARISON OF 2005 SPAR-H AND 1995 ASP HRA PSF MULTIPLIERS

\begin{tabular}{|c|c|c|c|c|c|c|}
\hline \multicolumn{2}{|c|}{2005 SPAR-H } & \multicolumn{3}{|c|}{1995 ASP HRA } & \multicolumn{2}{|c|}{$\begin{array}{c}\text { PSF Multiplier } \\
\text { (SPAR-H | ASP HRA) }\end{array}$} \\
\hline PSF & PSF Level & PSF & PSF Category & PSF Level & $\begin{array}{l}\text { Processing/ } \\
\text { Diagnosis }\end{array}$ & $\begin{array}{c}\text { Response/ } \\
\text { Action }\end{array}$ \\
\hline \multirow[t]{5}{*}{ Available Time } & Inadequate time & $\begin{array}{c}\text { Complexity, Stress, } \\
\text { Workload }\end{array}$ & $\begin{array}{l}\text { Low Threat and } \\
\text { Stress }\end{array}$ & $\begin{array}{l}\text { Inadequate } \\
\text { Time }\end{array}$ & $\begin{array}{c}\infty \mid \infty \\
(\text { See Note 1) }\end{array}$ & $\begin{array}{c}\infty \mid \infty \\
\text { (See Note 1) }\end{array}$ \\
\hline & Barely adequate time & & & & $\begin{array}{c}10 \mid \varnothing \\
\text { (See Note 2) }\end{array}$ & $\begin{array}{c}10 \mid \varnothing \\
\text { (See Note 2) }\end{array}$ \\
\hline & Nominal time & $\begin{array}{l}\text { Complexity, Stress, } \\
\text { Workload }\end{array}$ & $\begin{array}{l}\text { Low Threat and } \\
\text { Stress }\end{array}$ & Adequate Time & $1 \mid 1$ & $1 \mid 1$ \\
\hline & Extra time & & & & $\begin{array}{c}0.1 \mid \varnothing \\
\text { (See Note 2) }\end{array}$ & $\begin{array}{c}0.1 \mid \varnothing \\
\text { (See Note 2) }\end{array}$ \\
\hline & Expansive time & $\begin{array}{c}\text { Complexity, Stress, } \\
\text { Workload }\end{array}$ & $\begin{array}{l}\text { Low Threat and } \\
\text { Stress }\end{array}$ & Expansive Time & $\begin{array}{c}0.01 \mid 1 \\
\text { (See Note 3) } \\
\end{array}$ & $0.01 \mid 1$ \\
\hline \multirow[t]{3}{*}{ Stress/ Stressors } & Extreme & $\begin{array}{c}\text { Complexity, Stress, } \\
\text { Workload }\end{array}$ & $\begin{array}{c}\text { High Threat and } \\
\text { Stress }\end{array}$ & Adequate Time & $5 \mid 5$ & $\begin{array}{l}5 \mid 5 \\
\end{array}$ \\
\hline & High & $\begin{array}{l}\text { Complexity, Stress, } \\
\text { Workload }\end{array}$ & $\begin{array}{c}\text { High Threat and } \\
\text { Stress }\end{array}$ & Expansive Time & $2 \mid 2$ & $2 \mid 2$ \\
\hline & Nominal & $\begin{array}{l}\text { Complexity, Stress, } \\
\text { Workload }\end{array}$ & $\begin{array}{l}\text { Low Threat and } \\
\text { Stress }\end{array}$ & Adequate Time & $1 \mid 1$ & $1 \mid 1$ \\
\hline \multirow[t]{4}{*}{ Complexity } & Highly complex & $\begin{array}{c}\text { Complexity, Stress, } \\
\text { Workload }\end{array}$ & $\begin{array}{c}\text { High Threat and } \\
\text { Stress }\end{array}$ & Adequate Time & $\begin{array}{l}5 \mid 5 \\
\end{array}$ & $25 \mid 5$ \\
\hline & Moderately complex & $\begin{array}{l}\text { Complexity, Stress, } \\
\text { Workload }\end{array}$ & $\begin{array}{l}\text { High Threat and } \\
\text { Stress }\end{array}$ & Expansive Time & $2 \mid 2$ & $2 \mid 2$ \\
\hline & Nominal & $\begin{array}{l}\text { Complexity, Stress, } \\
\text { Workload }\end{array}$ & $\begin{array}{l}\text { Low Threat and } \\
\text { Stress }\end{array}$ & Adequate Time & $1 \mid 1$ & $1 \mid 1$ \\
\hline & Obvious diagnosis & & & & $\begin{array}{c}0.1 \mid \varnothing \\
\text { (See Note 2) } \\
\end{array}$ & \\
\hline \multirow{3}{*}{$\begin{array}{l}\text { Experience/ } \\
\text { Training }\end{array}$} & Low & Experience/ Training & Low Experience & Poor Training & $10 \mid 10$ & \begin{tabular}{l|l}
$3 \mid 10$ \\
.0
\end{tabular} \\
\hline & Nominal & Experience/ Training & Low Experience & Good Training & $1 \mid 1$ & $1 \mid 1$ \\
\hline & High & Experience/ Training & High Experience & Good Training & \begin{tabular}{l|l|}
0.5 & 0.5 \\
\end{tabular} & \begin{tabular}{l|l}
0.5 & 0.5 \\
\end{tabular} \\
\hline \multirow[t]{5}{*}{ Procedures } & Not available & Procedures & Procedures Absent & N/A & $50 \mid 10$ & $50 \mid 10$ \\
\hline & Incomplete & & & & $\begin{array}{c}20 \mid \varnothing \\
(\text { See Note 2) }\end{array}$ & $\begin{array}{c}20 \mid \varnothing \\
(\text { See Note 2) }\end{array}$ \\
\hline & Available, but poor & Procedures & Procedures Present & Poor Procedures & $5 \mid 5$ & $5 \mid 5$ \\
\hline & Nominal & Procedures & Procedures Present & $\begin{array}{c}\text { Good } \\
\text { Procedures }\end{array}$ & $1 \mid 1$ & $1 \mid 1$ \\
\hline & $\begin{array}{c}\text { Diagnostic/symptom } \\
\text { oriented }\end{array}$ & & & & $\begin{array}{c}0.5 \mid \varnothing \\
\text { (See Note 2) }\end{array}$ & \\
\hline \multirow[t]{4}{*}{$\begin{array}{c}\text { Ergonomics/ } \\
\text { HMI }\end{array}$} & " Missing/Misleading & & & & $\begin{array}{c}50 \mid \varnothing \\
(\text { See Note 2) }\end{array}$ & $\begin{array}{c}50 \mid \varnothing \\
(\text { See Note 2) }\end{array}$ \\
\hline & Poor & Ergonomics & Old Plant & $\begin{array}{c}\text { Poor } \\
\text { Ergonomics }\end{array}$ & $10 \mid 5$ & $10 \mid 5$ \\
\hline & Nominal & Ergonomics & Old Plant & $\begin{array}{c}\text { Good } \\
\text { Ergonomics }\end{array}$ & $1 \mid 1$ & $1 \mid 1$ \\
\hline & Good & Ergonomics & New Plant & $\begin{array}{c}\text { Good } \\
\text { Ergonomics }\end{array}$ & $0.5 \mid 0.4$ & $0.5 \mid 0.4$ \\
\hline \multirow[t]{3}{*}{ Fitness for Duty } & Unfit & "Fitness for Duty & Unfit & N/A & \begin{tabular}{ll|l|}
$\infty$ & 25
\end{tabular} & \begin{tabular}{c|c|}
$\infty$ & 25
\end{tabular} \\
\hline & Degraded Fitness & & & & $\begin{array}{c}5 \mid \varnothing \\
\text { (See Note 2) }\end{array}$ & $\begin{array}{c}5 \mid \varnothing \\
\text { (See Note 2) }\end{array}$ \\
\hline & Nominal & Fitness for Duty & Fit & $\mathrm{N} / \mathrm{A}$ & $1 \mid 1$ & $1 \mid 1$ \\
\hline \multirow[t]{3}{*}{ Work Processes } & Poor & Crew Dynamics & $\begin{array}{l}\text { Poor Crew } \\
\text { Dynamics }\end{array}$ & 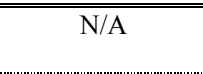 & 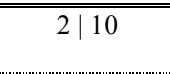 & \begin{tabular}{l|l|l}
5 \\
\end{tabular} \\
\hline & Nominal & Crew Dynamics & $\begin{array}{l}\text { Good Crew } \\
\text { Dynamics }\end{array}$ & $\mathrm{N} / \mathrm{A}$ & $1 \mid 1$ & $1 \mid 1$ \\
\hline & Good & & & & $\begin{array}{c}0.8 \mid \varnothing \\
\text { (See Note 2) }\end{array}$ & $\begin{array}{c}0.5 \mid \varnothing \\
\text { (See Note 2) }\end{array}$ \\
\hline
\end{tabular}

\section{Notes}

1. Multipliers are not used. Instead, the HEP is set to 1.0 for this PSF level.

2. This PSF level is not covered by the ASP HRA method.

3. The 2005 version of SPAR-H makes a distinction between At Power and Low Power or Shutdown in terms of the PSF multipliers. In practice, the only difference is that the multiplier for Expansive Time Diagnosis is given as a range of 0.1 to 0.01 for Low Power and Shutdown while only as a single multiplier of 0.01 for At Power. 
Joint 8th IEEE HFPP / 13th HPRCT

TABLE III. MAPPING OF SPAR-H (2005) TO THERP PSF MULTIPLIERS

\begin{tabular}{|c|c|c|c|c|c|}
\hline \multicolumn{4}{|c|}{ SPAR-H (NUREG/CR-6883) } & \multicolumn{2}{|c|}{ THERP (NUREG/CR-1278) } \\
\hline PSFs & PSF Levels & $\begin{array}{c}\text { HEP for } \\
\text { Diagnosis }^{1}\end{array}$ & $\begin{array}{l}\text { HEP for } \\
\text { Action }^{1}\end{array}$ & $\begin{array}{c}\text { HEP for } \\
\text { Diagnosis }^{2}\end{array}$ & $\begin{array}{l}\text { HEP for } \\
\text { Action }^{2}\end{array}$ \\
\hline \multirow{5}{*}{$\begin{array}{l}\text { Available } \\
\text { Time }\end{array}$} & Inadequate time & 1.0 (no multiplier) & 1.0 (no multiplier) & $1(20-1,1)$ & \\
\hline & Barely adequate time & $0.1(10)$ & $0.01(10)$ & $0.1(20-1,3)$ & \\
\hline & Nominal time & $0.01(1)$ & $0.001(1)$ & $0.01(20-1,4)$ & \\
\hline & Extra time & $0.001(0.1)$ & $0.0001(0.1)$ & $0.001(20-1,5)$ & \\
\hline & Expansive time & $0.0001(0.1-0.01)$ & $0.00001(0.01)$ & $0.0001(20-1,6)$ & \\
\hline \multirow{3}{*}{$\begin{array}{c}\text { Stress/ } \\
\text { Stressors }\end{array}$} & Extreme & $0.05(5)$ & $0.005(5)$ & 0.25 & $5 \times(20-16,6)^{3}$ \\
\hline & High & $0.02(2)$ & $0.002(2)$ & $2 \times(20-16,4)^{3}$ & $2 \times(20-16,4)^{3}$ \\
\hline & Nominal & $0.01(1)$ & $0.001(1)$ & $1 \times(20-16,2 \text { or } 3)^{3}$ & $1 \times(20-16,2 \text { or } 3)^{3}$ \\
\hline \multirow[t]{4}{*}{ Complexity } & Highly complex & $0.05(5)$ & $0.005(5)$ & & $0.005(20-23,6)$ \\
\hline & Moderately complex & $0.02(2)$ & $0.002(2)$ & & $0.002(20-23,4)$ \\
\hline & Nominal & $0.01(1)$ & $0.001(1)$ & & $0.001(20-23,3)$ \\
\hline & Obvious diagnosis & $0.001(0.1)$ & N/A & & $0.0001(20-23,1)$ \\
\hline \multirow{3}{*}{$\begin{array}{l}\text { Experience/ } \\
\text { Training }\end{array}$} & Low & $0.1(10)$ & $0.003(3)$ & $2 \times(20-16,7)^{3}$ & $2 \times(20-16,4 \text { or } 5)^{3}$ \\
\hline & Nominal & $0.01(1)$ & $0.001(1)$ & & \\
\hline & High & $0.05(0.5)$ & $0.0005(0.5)$ & & \\
\hline \multirow[t]{5}{*}{ Procedures } & Not available & $0.5(50)$ & $0.05(50)$ & & $0.05(20-7,5)$ \\
\hline & Incomplete & $0.2(20)$ & $0.02(20)$ & & $0.01(20-7,3)$ \\
\hline & Available, but poor & $0.05(5)$ & $0.005(5)$ & & $0.003(20-7,2)$ \\
\hline & Nominal & $0.01(1)$ & $0.001(1)$ & & $0.001(20-7,1)$ \\
\hline & Diagnostic/symptom oriented & $0.005(0.5)$ & N/A & & \\
\hline \multirow{4}{*}{$\begin{array}{l}\text { Ergonomics } \\
\text { / HMI }\end{array}$} & Missing/Misleading & $0.5(50)$ & $0.05(50)$ & & $0.05(20-12,6)$ \\
\hline & Poor & $0.1(10)$ & $0.01(10)$ & & $0.01(20-13,5)$ \\
\hline & Nominal & $0.01(1)$ & $0.001(1)$ & & $0.001(20-13,1)$ \\
\hline & Good & $0.005(0.5)$ & $0.0005(0.5)$ & & \\
\hline \multirow{3}{*}{$\begin{array}{l}\text { Fitness for } \\
\text { Duty }\end{array}$} & Unfit & 1.0 (no multiplier) & 1.0 (no multiplier) & & \\
\hline & Degraded Fitness & $0.05(5)$ & $0.005(5)$ & & \\
\hline & Nominal & $0.01(1)$ & $0.001(1)$ & & \\
\hline \multirow{3}{*}{$\begin{array}{c}\text { Work } \\
\text { Processes }\end{array}$} & Poor & $0.02(2)$ & $0.005(5)$ & & \\
\hline & Nominal & $0.01(1)$ & $0.001(1)$ & & \\
\hline & Good & $0.008(0.8)$ & $0.0005(0.5)$ & & \\
\hline
\end{tabular}

${ }^{1}$ SPAR-H Multiplier in parentheses

${ }^{2}$ THERP table and item number (where applicable) provided in parentheses

${ }^{3}$ THERP provides multipliers, not HEPs, for these PSF levels

method built on the CORE-Data [14] empirical database of HEP values. Fitness for Duty was delineated to two degraded levels beyond nominal performance. An "Unfit" level featured a multiplier set to infinity, or, more precisely, an automatic tagging of the HEP equal to 1.0. This keeps the conservative screening value adopted in the 1995 version of SPAR-H but makes the PSF treatment consistent with the treatment of the "Inadequate Time" level of the Available Time PSF. A new level was added for "Degraded Fitness" and given a multiplier of 5 . This value proved slightly more conservative than the multiplier suggested in HEART [10].

The Crew Dynamics PSF was relabeled Work Processes and redefined to encompass a broader range of activities including plant culture and management involvement in activities. Two non-nominal levels were adopted for this PSF. The negative influence was captured in the "Poor" Work Processes level and aligned with HEART values for Error Producing Condition (EPC) 21. The positive influence was captured in the "Good" Work Processes level and aligned with CREAM [11] values for the Common Performance Condition (CPC) called Adequacy of Organization.
Note that in two cases the processing/diagnosis and response/action multipliers differ for the same level in the revised SPAR-H. "Low" Experience/Training has a multiplier of 10 for processing/diagnosis and 3 for response/ action. For "poor" Work Processes, processing/diagnosis features a multiplier equal to 2 , while response/action has a multiplier equal to 5 at the same level. These values, like the positive influences that were not covered in the 1995 version of SPAR-H nor in THERP, represent refinements made through expert judgment based on the need to attenuate overly conservative values and accentuate effects that were undercounted previously. This process parallels the basis for all multiplier revisions in SPAR-H [13]. Where available, a mapping to THERP or other available HRA methods was performed. In a few cases as noted, however, it was necessary to extrapolate or estimate appropriate multiplier values.

\section{DISCUSSION}

HRA methods have proposed up to fifty PSFs [15]. SPAR-H attempts to provide reasonable coverage of the spectrum of human performance influences in nuclear power plant operations within the framework of the minimum 


\section{Joint 8th IEEE HFPP / 13th HPRCT}

reasonable number of PSFs. The decision to use first six PSFs and later eight PSFs was based on a review of thenavailable HRA methods in the early phase of SPAR-H development as well as ongoing feedback received by the SPAR-H Team from risk analysts at the US NRC. The SPAR-H quantification values used for the PSFs were based on available data within HRA, especially data provided in the THERP method [5].

The SPAR-H method provides a potent extension of THERP that allows the analyst flexibility and generalizability beyond narrowly defined tasks and scenarios. This approach does not guarantee valid HEP estimates. It does nonetheless provide a useful tool for categorizing and quantifying human contributions to risk and for facilitating risk-informed decision making.

This paper provides a mapping of the PSF multipliers in SPAR-H to primary data, especially those HEPs originating in THERP. This mapping improves the tractability of SPAR-H estimates. However, it must be remembered that the primary data sources for HRA are not infallible or infinitely generalizable. A quality HRA should not rely blindly on the estimates provided by a particular HRA method, be it SPAR-H or any other method. Rather, the HRA team should carefully consider NUREG-1792, Good Practices for HRA [16], which advises analysts to "evaluate the reasonableness of HEPs obtained" through "plant history, comparisons with results of other analyses, and qualitative understanding of the actions and their contexts by experts" (Good Practice 8).

\section{ACKNOWLEDGEMENTS}

The SPAR-H Method has had a number of significant contributors during its development. The authors particularly acknowledge J.C. Byers and D.I. Gertman of the then Idaho National Engineering Laboratory for their essential work in developing the ASP HRA and later SPAR$\mathrm{H}$ methods, and the late P. O'Reilly of the US Nuclear Regulatory Commission for his essential championing and sponsorship of the method. The authors have attempted accurately to reconstruct their historical development efforts in this article. The sole responsibility for any inaccuracies in this account resides with the present authors.

\section{DISCLAIMER}

This article was prepared as an account of work sponsored by an agency of the US Government under US Department of Energy Idaho Operations Contract DE-AC07-05ID14517. The opinions expressed in this paper are those of the authors and not of an agency of the US Government. Neither the US Government nor any agency thereof, nor any employee, makes any warranty, expressed or implied, or assumes any legal liability or responsibility for any third party's use, or the results of such use, of any information, apparatus, product, or process disclosed in this publication, or represents that its use by such third party would not infringe privately owned rights.

\section{REFERENCES}

[1] H.S. Blackman and J.C. Byers, ASP Human Reliability Methodology Development, INEL-95/0139, Idaho Falls: Idaho National Engineering Laboratory, April 1995.

[2] J.C. Byers, D.I. Gertman, S.G. Hill, H.S. Blackman, C.D. Gentillon, B.P. Hallbert, and L.N. Haney, Revision of the 1994 ASP HRA Methodology, INEEL/EXT-99-00041, Idaho Falls: Idaho National Engineering and Environmental Laboratory, January 1999.

[3] D. Gertman, H. Blackman, J. Byers, L. Haney, C. Smith, and J. Marble, The SPAR-H Method, NUREG/CR-6883, Washington, DC: US Nuclear Regulatory Commission, August 2005.

[4] R.L. Boring and D.I. Gertman, Atomistic and holistic approaches to human reliability analysis in the US power industry, Journal of the Safety and Reliability Society, vol. 25(2), pp. 21-37, 2005.

[5] A. D. Swain, and H. E. Guttman, Handbook of Human Reliability Analysis with Emphasis on Nuclear Power Plant Applications, NUREG/CR-1278, Washington, DC: US Nuclear Regulatory Commission, August 1983.

[6] US Nuclear Regulatory Commission, Reactor Safety Study: An Assessment of Accident Risks in US Commercial Nuclear Power Plants, WASH-1400, NUREG-75/014, Washington, DC: US Nuclear Regulatory Commission, October 1975.

[7] A.D. Swain, A Method for Performing a Human Factors Reliability Analysis, Monograph SCR-686, Sandia National Laboratories, Albuquerque, NM, 1963.

[8] B.J. Bell and A.D. Swain, A Procedure for Conducting a Human Reliability Analysis for Nuclear Power Plants, NUREG/CR-2254, Washington, DC: US Nuclear Regulatory Commission, May 1983.

[9] A. D. Swain, Accident Sequence Evaluation Program (ASEP), NUREG/CR-4772, Washington, DC: US Nuclear Regulatory Commission, February 1987.

[10] J.C. Williams, A data-based method for assessing and reducing human error to improve operational performance, Proceedings of the IEEE Fourth Conference on Human Factors and Power Plants, pp. 436-450, New York: IEEE, 1988.

[11] E. Hollnagel, Cognitive Reliability and Error Analysis Method (CREAM), Oxford: Elsevier, 1998.

[12] International Atomic Energy Agency, Protection System and Related Features in Nuclear Power Plants, Safety Series No. 50-SG-D3, Vienna: International Atomic Energy Agency, 1980.

[13] J.C. Byers, D.I. Gertman, S.G. Hill, H.S. Blackman, C.D. Gentillon, B.P. Hallbert, and L.N. Haney, Simplified plant analysis risk (SPAR) human reliability analysis (HRA) methodology: Comparisons with other HRA methods, In Proceedings of the 44th Annual Meeting of the Human Factors and Ergonomics Society (HFES 2000), vol. 3, pp. 177-180, 2000.

[14] S. Taylor-Adams, B. Lambert, R. Kennedy, and B. Kirwan, Task Data Used in the Validation of THERP, HEART, and JHEDI, Birmingham, UK: Vol. 3 Industrial Ergonomics Group.

[15] Y.H.J. Chang and A. Mosleh, Cognitive modeling and dynamic probabilistic simulation of operating crew response to complex system accidents, Part 2: IDAC performance influencing model, Reliability Engineering and System Safety, vol. 92, issue 8, pp. 10141040, 2007.

[16] A. Kolaczkowski, J. Forester, E. Lois, and S. Cooper, Good Practices for Implementing Human Reliability Analysis (HRA), NUREG-1792, Washington, DC: US Nuclear Regulatory Commission, April 2005. 